Schriftenreihe des Instituts für Angewandte Informatik / Automatisierungstechnik Universität Karlsruhe (TH) Band 2

Jan Christoph Martin

\title{
Ein Beitrag zur Integration von Sensoren in eine anthropomorphe künstliche Hand mit flexiblen Fluidaktoren
}

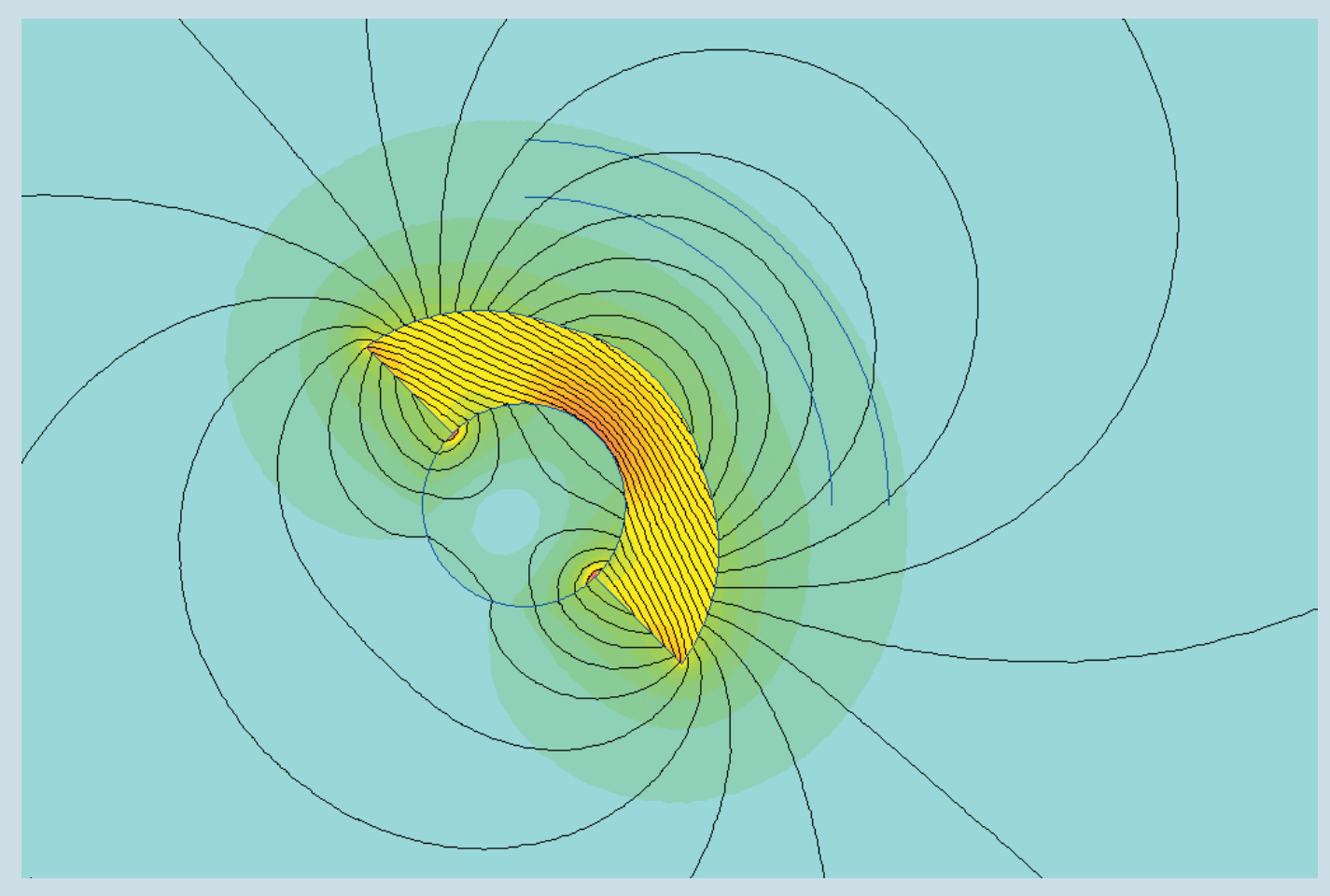


Dissertation, Universität Karlsruhe (TH), Fakultät für Maschinenbau, 2004

\section{Impressum}

Universitätsverlag Karlsruhe c/o Universitätsbibliothek

Straße am Forum 2

D-76131 Karlsruhe

www.uvka.de

(c) Universitätsverlag Karlsruhe 2004

Print on Demand

ISSN 1614-5267

ISBN 3-937300-24-4 


\section{Vorwort}

Die vorliegende Arbeit entstand während der drei Jahre meiner Tätigkeit am Institut für Angewandte Informatik des Forschungszentrums Karlsruhe.

Besonders danke ich Herrn Prof. Dr.-Ing. habil. Georg Bretthauer für die Möglichkeit, mit dieser Arbeit zur Entwicklung einer neuartigen künstlichen Hand für Prothetik und Robotik beizutragen. Er gab in Gesprächen viele wichtige Anregungen zu dieser Arbeit und trug immer wieder mit konstruktiver Kritik zu Manuskripten und Vortragsentwürfen bei.

Herrn Prof. Dr.-Ing. habil. Jürgen Wernstedt danke ich herzlichst für sein der Arbeit entgegen gebrachtes Interesse und die Übernahme des Korreferates.

Aus meiner Arbeitsgruppe danke ich insbesondere den Herren S. Schulz, C. Pylatiuk, A. Kargov und A. Ebersoldt, die mit ihrer Unterstützung beim Aufbau von Prototypen sowie ihren Ratschlägen und kritischen Hinweisen die Inhalte dieser Arbeit maßgeblich beeinflusst haben. Den Herren R. Mikut, S. Beck, A. Lehmann und D. Kraut danke ich für den regen Gedankenaustausch und die vertrauensvolle Zusammenarbeit. Sie trugen entscheidend zum Nachweis der Anwendbarkeit der Sensoren in Regelung und Überwachung bei.

Für ihre Hilfsbereitschaft und wertvolle Diskussionen danke ich auch meinen beiden Zimmerkollegen und Mitdoktoranden, Frau S. Mounier und Herrn T. Loose. Dank gilt ebenso den anderen Mitarbeitern des Instituts für Angewandte Informatik, die mit Rat und Tat am Gelingen dieser Arbeit mitgewirkt haben.

Schließlich möchte ich mich auch bei all denen in Familie und Freundeskreis bedanken, die mich zu diesem Projekt ermutigt und dabei auf vielfältige Weise immer wieder unterstützt haben. 


\title{
Kurzfassung
}

Neuartige Antriebsprinzipien wie flexible Fluidaktoren erlauben die Entwicklung leichter und vielgliedriger anthropomorpher Hände, deren Größe dem menschlichen Vorbild sehr nahe kommt. Sie schließen die Lücke zwischen Greifern mit eingeschränkter Beweglichkeit und sehr aufwändigen Roboterhänden für die Teleoperation. Ihre Einsatzgebiete sind sowohl Handprothesen als auch humanoide Serviceroboter.

Diese Arbeit trägt zur Entwicklung von Sensoren für solche Hände bei und zeigt ihre Anwendung am Beispiel von Regelungs- und Greifverfahren. Zusammengestellt werden die Aufgaben, Fähigkeiten und Anforderungen in Anlehnung an die Physiologie und Tätigkeiten der menschlichen Hand. Aus der Analyse und Modellierung des Aktorverhaltens werden weitere Randbedingungen für ein Sensorsystem hergeleitet. Nach der Darstellung der bereits in künstlichen Händen eingesetzten Messverfahren erfolgt die Auswahl, Auslegung und Optimierung von Winkelsensoren, taktilen Kraftsensoren und Sensoren zur Messung des Aktordrucks. Zur Übertragung der Sensorsignale an eine Steuerungseinheit werden unterschiedliche Leitungsmaterialien und Verkabelungsstrukturen bewertet.

Die Nutzung der Sensoren wird an Hand verschiedener Regelungsvarianten für einzelne und mehrere Gelenke im pneumatischen und hydraulischen Betrieb demonstriert. Abschließend werden die Funktionen einer Greifsteuerung als Schnittstelle zwischen der Gelenkregelung und einer übergeordneten Robotersteuerung diskutiert.

\begin{abstract}
Novel actuator principles such as flexible fluidic actuators allow for the development of lightweight artificial hands. Improvements are the compact realisation of a large number of articulations without exceeding the size of human hands. This progress closes the gap between simple grippers and highly sophisticated robot hands for teleoperation. Applications are prosthetic hands as well as humanoid service robots.

This thesis contributes to the development of sensors and control methods for such hands. The task and capability requirements are summarised with respect to the physiology and function of the human hand. Further requisites are derived from an analysis of the actuator behaviour. After demonstrating established sensing techniques in robot hands, sensors are selected, designed and optimised. The selected sensor types are used to determine joint angles, contact forces and actuator pressures. Different wiring materials and structures are evaluated for the transfer of sensor information to a control unit.

The use of sensor data is demonstrated with a number of control methods for single and multiple joints, both for pneumatic and hydraulic operation. Finally, the functions of a grasp control unit interfacing between joint control and high-level robot control are discussed.
\end{abstract}




\section{Inhaltsverzeichnis}

$\begin{array}{ll}\text { Vorwort } & 3\end{array}$

$\begin{array}{lr}\text { Kurzfassung } & 5\end{array}$

\begin{tabular}{lr}
\hline Inhaltsverzeichnis & 6
\end{tabular}

1 Einleitung 13

1.1 Bedeutung menschlicher Hände . . . . . . . . . . . . . . . . 13

1.2 Darstellung des Entwicklungsstandes . . . . . . . . . . . . . . . . 14

1.2.1 Greifer und künstliche Hände . . . . . . . . . . . . . . . . . . . 14

1.2.2 Gegenüberstellung der Einsatzgebiete . . . . . . . . . . . . 17

1.2 .3 Aktoren . . . . . . . . . . . . . . . . . 18

1.2 .4 Sensoren . . . . . . . . . . . . . . . . . . . . . . 19

1.2 .5 Planung, Steuerung und Regelung . . . . . . . . . . . . . . . 20

1.3 Offene Probleme . . . . . . . . . . . . . . . . . . . . . 21

1.4 Ziele und Aufgaben . . . . . . . . . . . . . . . . . 22

2 Anforderungen an humanoide Roboterhände 24

2.1 Physiologie der menschlichen Hand . . . . . . . . . . . . . . . . . 24

2.1.1 Anatomie und Biomechanik. . . . . . . . . . . . . . . . 24

2.1 .2 Gewebe und Haut . . . . . . . . . . . . . . . . . . 25

2.1 .3 Wahrnehmung . . . . . . . . . . . . . . . 26

2.2 Aktionen der menschlichen Hand . . . . . . . . . . . . . . . 28

2.2 .1 Definitionen . . . . . . . . . . . . . . . . . . . 28

2.2 .2 Ablauf von Handaktionen . . . . . . . . . . . . . . . . . . . . 29

2.2 .3 Klassifikation von Greifmustern . . . . . . . . . . . . . . 30

2.2.4 Programmieren durch Vormachen . . . . . . . . . . . . . . . . . . . 31

2.3 Einzelanforderungen . . . . . . . . . . . . . . . . 32

2.3.1 Aufgaben und Fähigkeiten . . . . . . . . . . . . . 32

2.3.2 Handkinematik . . . . . . . . . . . . . . . . . 33

2.3.3 Auswahl der Sensorklassen . . . . . . . . . . . . . . . . 34

2.3.4 Anforderungen an die Sensoren . . . . . . . . . . . . . . 34

$\begin{array}{llr}3 & \text { Flexible Fluidaktoren } & \mathbf{3 6}\end{array}$

3.1 Aufbau flexibler Fluidaktoren . . . . . . . . . . . . . . . . . 36

3.1 .1 Kontraktionsprinzip . . . . . . . . . . . . . . 36 
3.1 .2 Expansionsprinzip . . . . . . . . . . . . . 37

3.2 Kenngrößen und Modellierung des Gelenkaktors . . . . . . . . . . . 38

3.2 .1 Momentenkennfeld . . . . . . . . . . . . . . . . . . . . . . 38

3.2 .2 Volumenkennfeld . . . . . . . . . . . . . . . . . . . . . . . . . . . . . . . 49

3.3 Passive Momententrajektorien . . . . . . . . . . . . . . . . . . 41

3.3 .1 Pneumatischer Betrieb . . . . . . . . . . . . . . . . 42

3.3 .2 Hydraulischer Betrieb . . . . . . . . . . . . . . . . . 42

3.4 Medienabhängiges Steifigkeitsverhalten . . . . . . . . . . . . . . . 45

3.4.1 Pneumatischer Betrieb . . . . . . . . . . . . . . . . 45

3.4 .2 Hydraulischer Betrieb . . . . . . . . . . . . . . . 45

\begin{tabular}{|ll|l|}
\hline 4 & Auswahl und Optimierung der Sensoren & 47
\end{tabular}

4.1 Winkelsensoren . . . . . . . . . . . . . . . . . . . . 47

4.1 .1 Gelenkinterne Sensoren . . . . . . . . . . . . . . . . . . 48

4.1 .2 Externe Sensoren . . . . . . . . . . . . . . . . . . . . . . 49

4.1 .3 Anforderungen und Auswahl . . . . . . . . . . . . . . 50

4.1.4 Winkelmessung mit Magnetfeldsensoren . . . . . . . . . . . . 51

4.1 .5 Mechanische Integration . . . . . . . . . . . . . . . . . . . . . . . . . . . . 54

4.1 .6 Dimensionierung . . . . . . . . . . . . . . . . . . . 57

4.1 .7 Geometrieoptimierung des Magneten . . . . . . . . . . . . . . . . . 59

4.1.8 Aufbau und Test von Prototypen . . . . . . . . . . . . . 62

4.2 Taktile Sensoren . . . . . . . . . . . . . . . . . . . . . . . . . . 64

4.2.1 Kraft- und Drucksensoren . . . . . . . . . . . . . . . . . . . 66

4.2 .2 Mehrfeldsensoren . . . . . . . . . . . . . . . . 68

4.2 .3 Arraysensoren . . . . . . . . . . . . . . . . . . 71

4.2.4 Anforderungen und Auswahl . . . . . . . . . . . . . . . . . . . 73

$4.2 .5 \quad$ Kraftmessung mit FSR-Sensoren . . . . . . . . . . . . . . . 74

4.2 .6 Betätigung und mechanische Integration . . . . . . . . . . . 75

4.2.7 Messverfahren, Sensorkennlinie und Approximation . . . . . 77

4.2 .8 Messung von Greifkräften . . . . . . . . . . . . . . . . . . . . . 79

4.3 Momentensensoren . . . . . . . . . . . . . . . . . . . . . . 80

4.3 .1 Multiaxiale Kraft-Momenten-Sensoren . . . . . . . . . . . . 81

4.3 .2 Gelenkmomentsensoren . . . . . . . . . . . . . . . . . 83

4.3 .3 Anforderungen und Auswahl . . . . . . . . . . . . . . . 84

4.3.4 Druckmessung in flexiblen Fluidaktoren . . . . . . . . . . . . . 85

4.3 .5 Dimensionierung . . . . . . . . . . . . . . . . . . . 86

4.4 Verkabelung . . . . . . . . . . . . . . . . . . . . . . . . . 89

4.4 .1 Anforderungen . . . . . . . . . . . . . . . . . 89

4.4 .2 Leitermaterialien und Stecker . . . . . . . . . . . . . . . . . 90

4.4 .3 Verkabelungsstruktur . . . . . . . . . . . . . . . . . 93

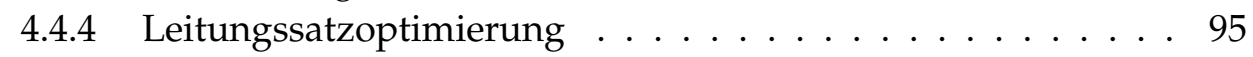

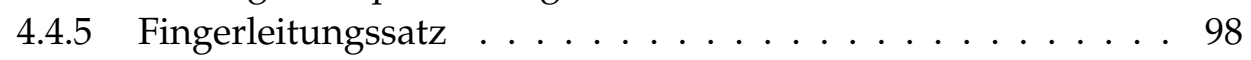

4.4 .6 Daumenleitungssatz . . . . . . . . . . . . . . . . . . . . . . . . . . . . . . . . . 99

4.4 .7 Zusammenfassung . . . . . . . . . . . . . . . 100 
5 Anwendung der Sensoren: Gelenkregelung und Greifen 101

5.1 Positionsregelung im pneumatischen Betrieb . . . . . . . . . . . . 101

5.1.1 Gelenkmodell . . . . . . . . . . . . . . . . . . . . . . . . . . . . 101

5.1 .2 Positionsregler . . . . . . . . . . . . . . . . . . 103

5.1 .3 Druckregelung . . . . . . . . . . . . . . . . . . . 105

5.2 Positionsregelung im hydraulischen Betrieb . . . . . . . . . . . . . 109

5.2 .1 Streckenmodell . . . . . . . . . . . . . . . . . . . . . . . 109

5.2 .2 Positionsregler . . . . . . . . . . . . . . . . . 110

5.3 Momentensteuerung . . . . . . . . . . . . . . . . . . . . 111

5.3 .1 Testumgebung . . . . . . . . . . . . . . . . 111

5.3.2 Statische Momentenbegrenzung . . . . . . . . . . . . . . 112

5.3.3 Dynamische Momentenbegrenzung . . . . . . . . . . . . . . 114

5.3 .4 Steifigkeitssteuerung . . . . . . . . . . . . . . . . . . . 115

5.3 .5 Kontakterkennung . . . . . . . . . . . . . . . . . . 117

5.4 Finger- und Gelenkkoordination . . . . . . . . . . . . . . 117

5.4.1 Hydraulischer Betrieb . . . . . . . . . . . . . . . . . . . 117

5.4 .2 Pneumatischer Betrieb . . . . . . . . . . . . . . . . . . . . . . . 119

5.4 .3 Fingerpriorisierung . . . . . . . . . . . . . . . . . . . . 119

5.5 Steuerungsstruktur für Hände und Arme . . . . . . . . . . . . . . 120

5.6 Greifplanung und -koordination . . . . . . . . . . . . . . . 121

5.6 .1 Modellbasierte Greifplanung . . . . . . . . . . . . . . . . . . 121

5.6.2 Greifplanung für einen humanoiden Roboter . . . . . . . . . . . . . . . . . . . . . .

5.6.3 Generierung von Greifmusterdaten . . . . . . . . . . . . . 122

5.7 Handsteuerung $\ldots \ldots \ldots \ldots . \ldots \ldots \ldots$

5.8 Hand- Arm-Koordination . . . . . . . . . . . . . . . . . 123

6 Zusammenfassung und Ausblick $\quad 126$

$\begin{array}{lr}\text { Literaturverzeichnis } & 129\end{array}$

\begin{tabular}{ll}
\hline Abbildungsverzeichnis & 146
\end{tabular}

\begin{tabular}{lr}
\hline Tabellenverzeichnis & 149
\end{tabular}

$\begin{array}{llr}7 & \text { Anhang } & \mathbf{1 5 0}\end{array}$

7.1 Technische Daten der Sensoren . . . . . . . . . . . . . . . . 150

7.1 .1 Hallsensoren . . . . . . . . . . . . . . . . . . . . 150

7.1 .2 Magnete . . . . . . . . . . . . . . . . . . . . . 151

7.1 .3 IEE FSR-Sensoren . . . . . . . . . . . . . . . . . . . . 151

7.1 .4 Drucksensoren . . . . . . . . . . . . . . . . . . 152

7.2 Verkabelung . . . . . . . . . . . . . . . . . 153

$7.2 .1 \quad$ Finger mit zwei Freiheitsgraden . . . . . . . . . . . . . . 153

7.2.2 Daumen mit drei Freiheitsgraden . . . . . . . . . . . . . . 154

7.3 Genetischer Algorithmus . . . . . . . . . . . . . . . . . . . . . 155 


\section{Symbolverzeichnis}

Die wichtigsten Abkürzungen, Symbole, Formelzeichen und Einheiten sind an dieser Stelle thematisch sortiert zusammengefasst. Druckangaben sind, soweit nicht anders angegeben, relativ zum Umgebungsdruck.

\section{Flexible Fluidaktoren}

\begin{tabular}{l|l} 
Formelzeichen & Bedeutung \\
\hline$\varphi$ & Gelenkwinkel $\left[1^{\circ}\right]$ \\
$p$ & Aktordruck $[\mathrm{Pa}]$ \\
$p_{0}$ & Umgebungsdruck $[\mathrm{Pa}]$ \\
\hline$M$ & Drehmoment $[\mathrm{Nm}]$ \\
$M_{\mathrm{A}}$ & Aktormoment $[\mathrm{Nm}]$ \\
$h$ & Höhe der Aktormitte über dem Gelenk $[\mathrm{mm}]$ \\
$r_{0}$ & Aktorradius bei Umgebungsdruck $[\mathrm{mm}]$ \\
$l_{0}$ & Länge des Aktors bei gestrecktem Gelenk $[\mathrm{mm}]$ \\
$A$ & Aktorquerschnittsfläche $\left[\mathrm{mm}^{2}\right]$ \\
$a_{00} \ldots a_{11}$ & Aktorparameter für Momentenkennfeld \\
\hline$V$ & Volumen $\left[\mathrm{cm}^{3}\right]$ \\
$\left.V_{\mathrm{A}}\right]$ & Aktorvolumen $\left[\mathrm{cm}^{3}\right]$ \\
$E$ & relative druckabhängige Flächenausdehnung $\left[\frac{1}{\mathrm{~Pa}}\right]$ \\
$b_{0} \ldots b_{3}$ & Aktorparameter für Volumenkennfeld \\
\hline$T$ & Temperatur $[\mathrm{K}]$ \\
$m$ & Masse $[\mathrm{kg}]$ \\
$c$ & Federkonstante $\left[\frac{\mathrm{N}}{\left.1^{\circ}\right]}\right.$ \\
$k$ & Nachgiebigkeitskonstante $\left[\frac{1^{\circ}}{N}\right]$ \\
\hline
\end{tabular}

\section{Sensoren}

\begin{tabular}{l|l} 
Formelzeichen & Bedeutung \\
\hline$B, B_{0}$ & magnetische Flussdichte $[T]$ \\
$B_{\mathrm{x}}, B_{\mathrm{y}}$ & magnetische Flussdichte in $\mathrm{x} / \mathrm{y}$-Richtung $[T]$ \\
$B_{\mathrm{n}}, B_{\mathrm{t}}$ & normale/tangentiale magnetische Flussdichte $[T]$ \\
$U_{\mathrm{A}}$ & Ausgangsspannung $[\mathrm{V}]$ \\
$U_{\mathrm{V}}$ & Versorgungsspannung $[\mathrm{V}]$ \\
\hline
\end{tabular}




\begin{tabular}{|c|c|}
\hline Formelzeichen & Bedeutung \\
\hline$w_{i}$ & Approximationsparameter für Winkelsensor \\
\hline$\delta$ & mittlerer Winkelfehler $\left[1^{\circ}\right]$ \\
\hline$r$ & Magnetradius $[\mathrm{mm}]$ \\
\hline$\beta_{i}$ & Winkelposition des Stützpunkts $\left[1^{\circ}\right]$ \\
\hline$g_{i}$ & Radius am Stützpunkt (Genotyp) $[\mathrm{mm}]$ \\
\hline$\sigma$ & Weiteparameter für RBF-Netzwerk [ ] \\
\hline$\hat{\varphi}$ & geschätzter Winkel $\left[1^{\circ}\right]$ \\
\hline$f$ & Fitness \\
\hline$R_{\mathrm{S}}, R_{\mathrm{T}}$ & Sensor- und Teilerwiderstand $[\Omega]$ \\
\hline$U_{\mathrm{S}}, U_{\mathrm{T}}$ & Spannung an Sensor und Teilerwiderstand $[V]$ \\
\hline$F$ & Kraft $[N]$ \\
\hline$\varrho_{\mathrm{Cu}}$ & spezifischer Widerstand von Kupfer $[\Omega m]$ \\
\hline$j$ & Gelenkindex [ ] \\
\hline$k_{\mathrm{L} j}, k_{\mathrm{R} j}$ & Anzahl Kontakte an der linken/rechten Seite [ ] \\
\hline$l_{\mathrm{L} j}, l_{\mathrm{R} j}$ & Anzahl Leitungen an der linken/rechten Seite [ ] \\
\hline$n_{\mathrm{K}}$ & Kontaktanzahl [ ] \\
\hline$v_{\mathrm{K}}$ & Kontaktverteilung [ ] \\
\hline$s_{\mathrm{K}}$ & Kontaktsymmetrie [ ] \\
\hline$n_{\mathrm{L}}$ & Leitungsanzahl [ ] \\
\hline$s_{\mathrm{L}}$ & Leitungssymmetrie [ ] \\
\hline$l_{\mathrm{F}}$ & Fingerlänge [ ] \\
\hline$w$ & Gewichtungsfaktor [ ] \\
\hline$b$ & Bewertung einer Variante [ ] \\
\hline
\end{tabular}

\section{Steuerung und Regelung}

\begin{tabular}{l|l} 
Formelzeichen & Bedeutung \\
\hline$\varphi$ & Gelenkwinkel $\left[1^{\circ}\right]$ \\
$\dot{\varphi}$ & Winkelgeschwindigkeit des Gelenks $\left[\frac{1^{\circ}}{s}\right]$ \\
$\ddot{\varphi}$ & Winkelbeschleunigung des Gelenks $\left[\frac{1^{\circ}}{s^{2}}\right]$ \\
$\varphi_{\text {ist }}$ & Istwinkel des Gelenks $\left[1^{\circ}\right]$ \\
$\varphi_{\text {soll }}$ & Sollwinkel des Gelenks $\left[1^{\circ}\right]$ \\
$e_{\varphi}$ & Winkelfehler $\left[1^{\circ}\right]$ \\
$A \cdot \Delta \varphi_{\max }$ & Winkelabweichung durch Viskoelastizität $\left[1^{\circ}\right]$ \\
\hline$p$ & Druck $[P a]$ \\
$p_{\text {ist }}$ & Istdruck des Aktors $[\mathrm{Pa}]$ \\
$p_{\text {soll }}$ & Solldruck des Aktors $[\mathrm{Pa}]$ \\
$e_{\mathrm{p}}$ & Druckabweichung $[\mathrm{Pa}]$ \\
$p_{\mathrm{V}}$ & Versorgungsdruck $[\mathrm{Pa}]$ \\
$p_{\max }$ & Maximaldruck $[\mathrm{Pa}]$ \\
$\Delta p$ & Druckdifferenz am Ventil $[\mathrm{Pa}]$ \\
\hline
\end{tabular}




\begin{tabular}{|c|c|}
\hline Formelzeichen & Bedeutung \\
\hline$M_{\text {Stör }}$ & Störmoment durch Kontakt [ $\mathrm{Nm}]$ \\
\hline & viskose Reibung des Gelenks $\left[\frac{\mathrm{Nm} \cdot \mathrm{s}}{1^{\circ}}\right]$ \\
\hline$J$ & Massenträgheit des Gelenks $\left[\frac{\mathrm{Nm} \cdot \mathrm{s}^{2}}{1^{\circ}}\right]$ \\
\hline$U_{\mathrm{p}}, U_{\mathrm{pP}}, U_{\mathrm{p} j}$ & Spannung vom Drucksensor (der Pumpe, des Gelenks $j$ ) $[V]$ \\
\hline$U_{\varphi}, U_{\varphi j}$ & Spannung vom Winkelsensor (des Gelenks $j$ ) $[V]$ \\
\hline$U_{\mathrm{V}}, U_{\mathrm{V} j}$ & Ventil-Ansteuersignal (des Gelenks $j$ ) $[V]$ \\
\hline$k_{\mathrm{R}}$ & Reglerverstärkung [ ] \\
\hline$T_{\mathrm{N}}$ & Nachstellzeit des I-Reglers \\
\hline$T_{\mathrm{V}}$ & Vorhaltezeit des D-Reglers \\
\hline$T_{\mathrm{Z}}$ & Verzögerung des D-Reglers \\
\hline$T_{\mathrm{A}}$ & Abtastzeit des Reglers $[s]$ \\
\hline$t$ & Zeit $[s]$ \\
\hline$m$ & Masse $[\mathrm{kg}]$ \\
\hline$\dot{m}$ & Massenstrom $\left[\frac{\mathrm{kg}}{\mathrm{s}}\right]$ \\
\hline$\mu$ & Verlustbeiwert des Ventils [ ] \\
\hline$A, A_{0}$ & Querschnittsfläche des Ventils $\left[\mathrm{m}^{2}\right]$ \\
\hline$\alpha$ & Öffnungsgrad des Ventils [ ] \\
\hline$\varrho, \varrho_{0}$ & Dichte, Dichte bei Normaldruck $\left[\frac{\mathrm{kg}}{\mathrm{m}^{3}}\right]$ \\
\hline$R$ & allgemeine Gaskonstante $\left[\frac{\mathrm{J}}{\mathrm{kg} \cdot \mathrm{K}}\right]$ \\
\hline$T, T_{\mathrm{R}}$ & Temperatur, Raumtemperatur $[K]$ \\
\hline$T_{\text {ein }}, T_{\text {aus }}$ & Öffnungs- und Schließverzögerung des Ventils $[s]$ \\
\hline$T_{\mathrm{an}}, T_{\mathrm{eff}}$ & Ansteuerdauer, effektive Öffnungsdauer $[\mathrm{ms}]$ \\
\hline$x, x_{V}, x_{P}$ & PWM-Tastverhältnis (Ventil, Pumpe) [ ] \\
\hline$T_{\mathrm{p}}$ & PWM-Periodendauer $[s]$ \\
\hline$S$ & Hydraulische Kapazität $\left[\frac{m^{3}}{P a}\right]$ \\
\hline$\dot{V}_{\mathrm{P}}, \dot{V}_{\mathrm{V}}, \dot{V}_{\mathrm{A}}$ & Volumenstrom (Pumpe, Ventil, Aktor) $\left[\frac{\mathrm{m}^{3}}{\mathrm{~s}}\right]$ \\
\hline$F_{1}, F_{2}$ & proximal und distal gemessene Kraft $[N]$ \\
\hline$l_{1}, l_{2}$ & Gelenkabstand des proximalen und distalen Kraftsensors $[\mathrm{m}]$ \\
\hline$M_{\max }$ & Maximalmoment $[\mathrm{Nm}]$ \\
\hline$M_{\text {max }, \text { statisch }}$ & statisches Maximalmoment $[\mathrm{Nm}]$ \\
\hline$M_{\text {max }, \text { dynamisch }}$ & dynamisches Maximalmoment $[\mathrm{Nm}]$ \\
\hline$M_{\max , \text { Feder }}$ & steifigkeitsabhängiges Maximalmoment $[\mathrm{Nm}]$ \\
\hline$M(\varphi, p), M_{\mathrm{FSR}}$ & Moment aus Aktormodell/ Kraftmessung [ $\mathrm{Nm}]$ \\
\hline$\Delta M(\varphi, p)$ & Momentenfehler aus Aktormodell $[\mathrm{Nm}]$ \\
\hline$\Delta M_{\mathrm{FSR}}$ & Momentenfehler aus Kraftmessung [ $\mathrm{Nm}]$ \\
\hline$E_{\mathrm{pos}}, E_{\mathrm{neg}}$ & positive/ negative Fehlersumme [ ] \\
\hline$r_{\mathrm{P}}$ & Gewichtungsfaktor für Umschaltung der Pumpenrichtung [ ] \\
\hline
\end{tabular}

\section{Konstanten}

\begin{tabular}{l|l|l} 
Formelzeichen & Wert & Bedeutung \\
\hline$R$ & $287,2 \frac{\mathrm{J}}{\mathrm{kg} \cdot \mathrm{K}}$ & allgemeine Gaskonstante \\
$T_{\mathrm{R}}$ & $296 \mathrm{~K}$ & Raumtemperatur \\
$\varrho_{0}$ & $1,29 \frac{\mathrm{kg}}{\mathrm{m}^{3}}$ & Dichte von Luft bei Normaldruck \\
\hline
\end{tabular}




\begin{tabular}{l|l} 
Abkürzungen & \\
Abkürzung & Bedeutung \\
\hline RBF & Radiale Basisfunktion, radial basis function \\
FSR & force sensing resistor \\
PVF $_{2}$, PVDF & Polyvinylidenfluorid \\
FBC & flexible bulk cable \\
FFC & flexible flat cable \\
FPC & flexible printed circuit \\
PCB & printed circuit board \\
\hline DOF & degree of freedom, Freiheitsgrad \\
PWM & Pulsweitenmodulation \\
FGA & Führungsgrößenaufschaltung \\
TCP & tool center point \\
\hline
\end{tabular}




\section{Einleitung}

Die menschliche Hand ist bei der Entwicklung künstlicher Hände sowohl für die Robotik als auch für die Prothetik immer noch ein unerreichtes Vorbild an Beweglichkeit, Geschick und Greifsicherheit. Dieses Kapitel gibt einen Überblick über Greifer in Robotik und Prothetik, die eingesetzten Aktoren und Sensoren sowie der Planung und Ausführung von Greifvorgängen. Abschließend werden aus den hierbei erkannten offenen Problemen die Ziele dieser Arbeit definiert.

\subsection{Bedeutung menschlicher Hände}

Die Hände dienen dem Menschen in vielerlei Hinsicht: Sie bieten die Möglichkeit, Gegenstände auf unterschiedlichste Weise zu greifen und als Werkzeuge zu nutzen. Gleichzeitig sind sie wichtige aktive Sinnesorgane, mit denen der Mensch seine Umwelt wahrnimmt, erforscht und begreift. Nicht zuletzt ist die Gestik der Hände ein wichtiger Teil der nichtverbalen menschlichen Kommunikation.

Schon Anaxagoras von Klazomenai (ca. 500-428 v. Chr.) verbindet die Hände, die den Menschen von allen anderen Lebewesen unterscheiden, ursächlich mit der Entwicklung der menschlichen Intelligenz. Aristoteles (384-322 v. Chr.) argumentiert dagegen, dass erst die Intelligenz es dem Menschen erlaube, die Hand als Werkzeug der Werkzeuge geschickt einzusetzen.

In der industriellen Robotik finden sich bisher statt vielfingriger Hände meist nur Werkzeugwechselsysteme oder Greifer mit wenigen Freiheitsgraden, die auf bestimmte Aufgaben spezialisiert sind. Dies setzt jedoch klar definierte Arbeitsumgebungen für den Roboter und eine Abstimmung von Greifer und zu greifendem Objekt aufeinander voraus. Dieser Aufwand ist nur bei sich oft wiederholenden Aufgaben wie z.B. in der automatisierten Produktion zu rechtfertigen.

Soll ein mobiler Roboter nun immer wieder wechselnde Aufgaben in für den Menschen geschaffenen Umgebungen übernehmen, so sind statt der spezialisierten Greifer flexibler einsetzbare Hände gefordert. Sie sind wie beim Menschen ein entscheidender Schritt für die Entwicklung von intelligenten Robotern, die auch in unstrukturierten Umgebungen sicher und zuverlässig arbeiten können. Hierfür ist die menschliche Hand ein sehr gutes Vorbild, insbesondere da sich der Mensch die meisten Gegenstände in seiner Umgebung an die Anatomie und die Fertigkeiten seiner Hände angepasst hat.

Ist darüber hinaus auch die direkte Interaktion des Roboters mit dem Menschen gefordert, gewinnen weitere, über die reine Anatomie hinaus gehende Faktoren wie die Nachgiebigkeit der Finger und eine elastische Hautoberfläche essen- 
tielle Bedeutung, da sie den Menschen vor Schaden schützen. Zu ergänzen ist dies mit Sensoren sowie Steuerungs- und Regelungsverfahren, die die kontrollierte Bewegung der Hand und ein sicheres Greifen ermöglichen.

Bis heute ist die Herausforderung der Entwicklung von menschenähnlichen künstlichen Händen nicht zufrieden stellend gelöst und kaum eine der in der Vergangenheit gebauten Roboterhände ist allgemein einsetzbar oder gar kommerziell verfügbar. Fortschritte sind dabei in allen an einer solchen Entwicklung beteiligten Fachgebieten wie Mechanik, Antriebe, Sensoren, Regelung und Steuerung gefordert und nur ein enges Ineinandergreifen dieser Disziplinen wird zu entscheidenden Weiterentwicklungen und einer verbesserten Integration der einzelnen Komponenten und Funktionen führen.

\subsection{Darstellung des Entwicklungsstandes}

In diesem Abschnitt werden die unterschiedlichen Zielstellungen und der Stand der Entwicklung künstlicher Hände, ihrer Antriebe und Sensoren sowie verschiedene Ansätze zur Greifplanung, Steuerung und Regelung solcher Hände betrachtet.

\subsubsection{Greifer und künstliche Hände}

Im Folgenden werden für verschiedene Gruppen künstlicher Hände typische Entwicklungen vorgestellt, für weiter gehende Übersichten und Vergleiche wird auf [17, 135] verwiesen.

\section{Industrielle Robotergreifer}

In der industriellen Robotik mit sich häufig wiederholenden Aufgaben in streng abgegrenzten und genau definierten Umgebungen werden heute spezialisierte Greifer mit wenigen Freiheitsgraden und minimaler Sensorik eingesetzt (Abbildung 1.1 links). Greifer und zu greifendes Objekt sind aufeinander abgestimmt, so wird die für Produktionsumgebungen geforderte Zuverlässigkeit erreicht [191].

\section{Mehrfingergreifer}

Roboterhände mit mehr Freiheitsgraden und mehrgelenkigen Fingern sind meist nicht am Vorbild des Menschen orientiert, sondern nach kinematischen Aspekten unter Berücksichtigung der verwendeten Aktortechnik optimiert. Als Beispiele hierfür können z.B. die Karlsruher Hand II (Abbildung 1.1 Mitte) [40, 41, 43, 52, 55, 56, 155], die TUM-Hand [128, 195], die Barrett Hand [185] und die Stanford/JPLHand [162] genannt werden. Das Ziel dieser Entwicklungen ist die Objektmanipulation mit den Fingern, also die kontrollierte Bewegung eines Objekts relativ zur Handwurzel. 

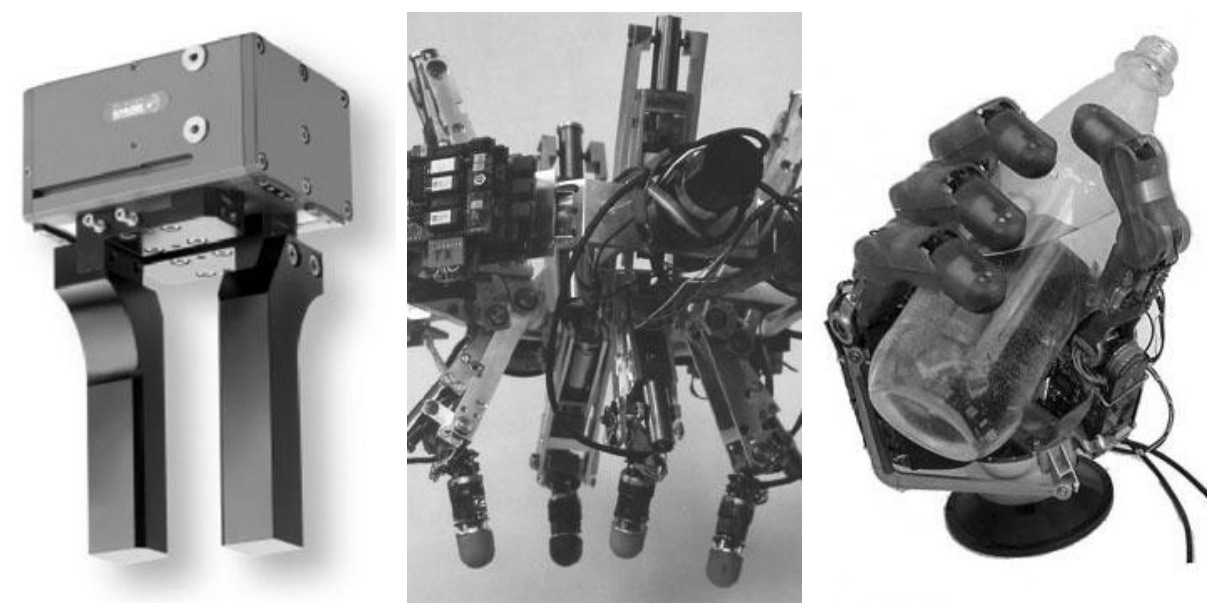

Abbildung 1.1: Beispiele für Greifer und eine anthropomorphe Hand: Pneumatischer Greifer Schunk PFH40, Karlsruher Hand II, DLRHand II (v.l.n.r.)

\section{Teleoperation}

In der Teleoperation gewinnt die anthropomorphe, also menschenähnliche Gestaltung der Roboterhände entscheidende Bedeutung, da sie nicht nur die Übertragung der mit einem Datenhandschuh erfassten Handbewegungen auf die Roboterhand, sondern auch eine Kraftrückkopplung an den Bediener deutlich vereinfacht oder überhaupt erst möglich macht. Ziel solcher Arbeiten ist es, aus der Ferne in unstrukturierten, für den Menschen gefährlichen Umgebungen arbeiten zu können. Für diese Aufgaben wurden z.B. die DLR-Hand II (Abbildung 1.1 rechts) [22, 23, 24], die Robonaut-Hand der NASA [120], die DIST-Hand [15, 25] und die LMS-Hand [60] entwickelt. Manche dieser Hände sind aus Gründen der Einfachheit nur teilanthropomorph ausgelegt und verzichten z.B. auf den kleinen Finger oder Größenunterschiede zwischen den einzelnen Fingern.

\section{Hände humanoider Roboter}

Die Hände der bisher realisierten humanoiden Roboter sind meist auf einen einzigen angetriebenen Freiheitsgrad beschränkt, sie sind dabei wie bei ARMAR [9] oft an industrielle Greifer angenähert. Beim japanischen HRP-2 [72] wird die Menschenähnlichkeit auf einen einer geschlossenen Fingerfläche fest opponierten Daumen reduziert (Abbildung 1.2 links). Ein weiterer Ansatz wird in der KarlsruheTUAT-Hand [59] (Abbildung 1.2 Mitte) oder bei den Händen von ASIMO [8] verfolgt, diese sind als so genannte underactuated hands ausgeführt. Dabei sind mehrere Freiheitsgrade über einen Kopplungsmechanismus wie z.B. Hebel und Seilzüge miteinander verbunden und über einen einzelnen Aktor angetrieben. Die Regelung dieser Hände wird meist als ein zusätzlicher Freiheitsgrad des Arms betrachtet. 

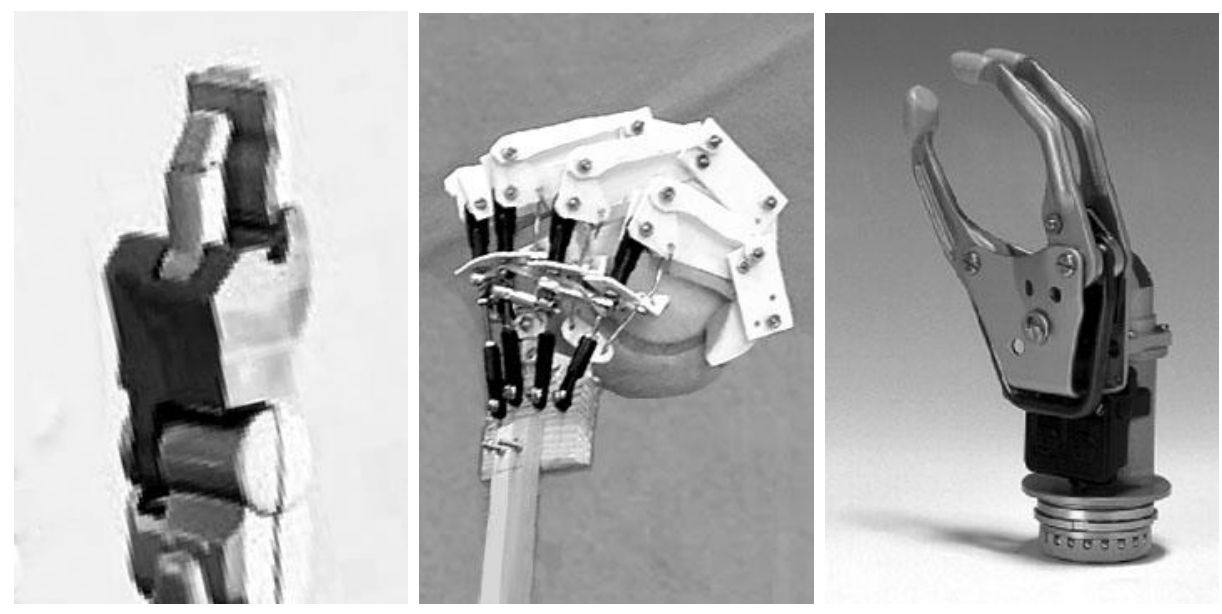

Abbildung 1.2: Beispiele für humanoide Roboterhände und eine Handprothese: HRP-2-Hand, Karlsruhe-TUAT-Hand, Otto Bock Elektrohand (v.l.n.r.).

\section{Handprothesen}

Im Kontext künstlicher menschenähnlicher Hände sind auch Handprothesen zu betrachten. Sie verhelfen einem Menschen, dem durch einen Unfall oder bereits von Geburt an eine Hand oder auch Teile eines Arms fehlen, zu einer größeren Selbstständigkeit im täglichen Leben. Neben passiven kosmetischen Handprothesen, die nur die Erscheinung der Hand wieder herstellen, stellen aktive Prothesen mit einem oder mehreren Freiheitsgraden auch einen Teil der Funktionen der fehlenden Hand wieder her.

Eigenkraftprothesen sind dabei von der Muskulatur des Menschen z.B. über ein Bowdenzugsystem von der gegenüberliegenden Schulter angetrieben [16]. Der Antrieb der moderneren Fremdkraftprothesen erfolgt meist durch batteriebetriebene Gleichstrommotoren [147, 148, 149]. Deren Ansteuerung erfolgt myoelektrisch, also über im Prothesenschaft angebrachte Oberflächenelektroden, die die von der Kontraktion der Restmuskulatur herrührenden Potentialunterschiede der Haut aufnehmen. Typische Greifer sind entweder als funktionale Haken mit einem Freiheitsgrad oder als Dreifingergreifer mit einem beweglichen, fest opponierten Daumen ausgeführt (Abbildung 1.2 rechts). Mit der geringen Anzahl an Antrieben ist auch die ebenso geringe Anzahl unterschiedlicher Bewegungen verbunden, dies führt zu einem unnatürlichen Aussehen der Greifhaltungen.

Derzeit wird in unterschiedlichen Forschungsprojekten wie z.B. der RTRHand [27, 28], der Dextra-Hand [32, 33], der Southampton-Remedi-Hand [104] untersucht, wie sich die Anzahl der Antriebe und Freiheitsgrade in Prothesen erhöhen lässt, um unterschiedliche Greifmuster und ein menschenähnlicheres Greifen zu ermöglichen. Neben der Realisierung der veränderten Antriebstechnik ist das Problem der Ansteuerung zu lösen, da die wenigen möglichen Kanäle einer myoelektrischen Steuerung nicht zum direkten Ansprechen der einzelnen Freiheitsgra- 
de verwendet werden können.

\section{FZK-Hand}

Einen neuartigen Ansatz für künstliche anthropomorphe Hände verwendet die am Institut für Angewandte Informatik des Forschungszentrums Karlsruhe entwickelte Hand [166, 172]. Sie wird von flexiblen Fluidaktoren angetrieben und kann sowohl in der Robotik (Abbildung 1.3, links) als auch als Handprothese (Abbildung 1.3, rechts) eingesetzt werden. Besonderheit dieser sowohl pneumatisch als auch hydraulisch einsetzbaren Aktoren ist ihre in den folgenden Kapiteln ausführlicher behandelte Nachgiebigkeit.
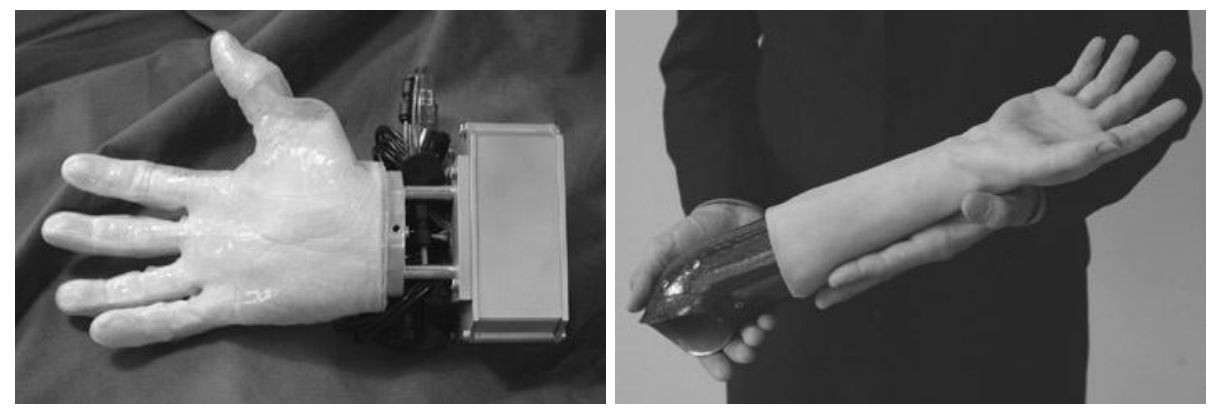

Abbildung 1.3: FZK-Hand: links als Konzept für eine modulare Roboterhand [125], rechts als Funktionsmuster einer Prothese [13].

\subsubsection{Gegenüberstellung der Einsatzgebiete}

Die im letzten Abschnitt vorgestellten Hände und Greifer mit ihren unterschiedlichen Zielstellungen und Einsatzgebieten lassen sich hinsichtlich ihrer Abhängigkeit vom Menschen als Bediener, ihrer Menschenähnlichkeit und ihrer Beweglichkeit, also der Anzahl an Gelenken und Freiheitsgraden einordnen (Abbildung 1.4).

Die menschliche Hand ist hier als Referenz anzusehen: sie ist über Nervenbahnen mit dem Gehirn verbunden und damit direkt unserem Willen unterworfen. Obwohl manche künstliche Hand ihr hinsichtlich Beweglichkeit und Geschick nahe kommt, ist ihre Leistungsfähigkeit auch hier unerreicht. Als Extrem auf der anderen Seite ist ein einfacher industrieller Greifer zu sehen, der mit einem einzigen angetriebenen Freiheitsgrad über eine Ablaufsteuerung immer wieder den gleichen, einmal von einem Bediener programmierten Arbeitsablauf ausführt.

Dazwischen sind die anderen Anwendungsfälle einzusortieren: Die Entwicklung von Mehrfingergreifern zielt auf die möglichst generische Planung und Ausführung von autonomen Greif- und Manipulationsaufgaben. Auf der anderen Seite sind die Hände in der Teleoperation mit einem sehr menschenähnlichen Verhalten höchstens als teilautonom zu bewerten, da sie über einen Datenhandschuh als Eingabegerät und eine Datenleitung direkt an einen Bediener gekoppelt sind. 


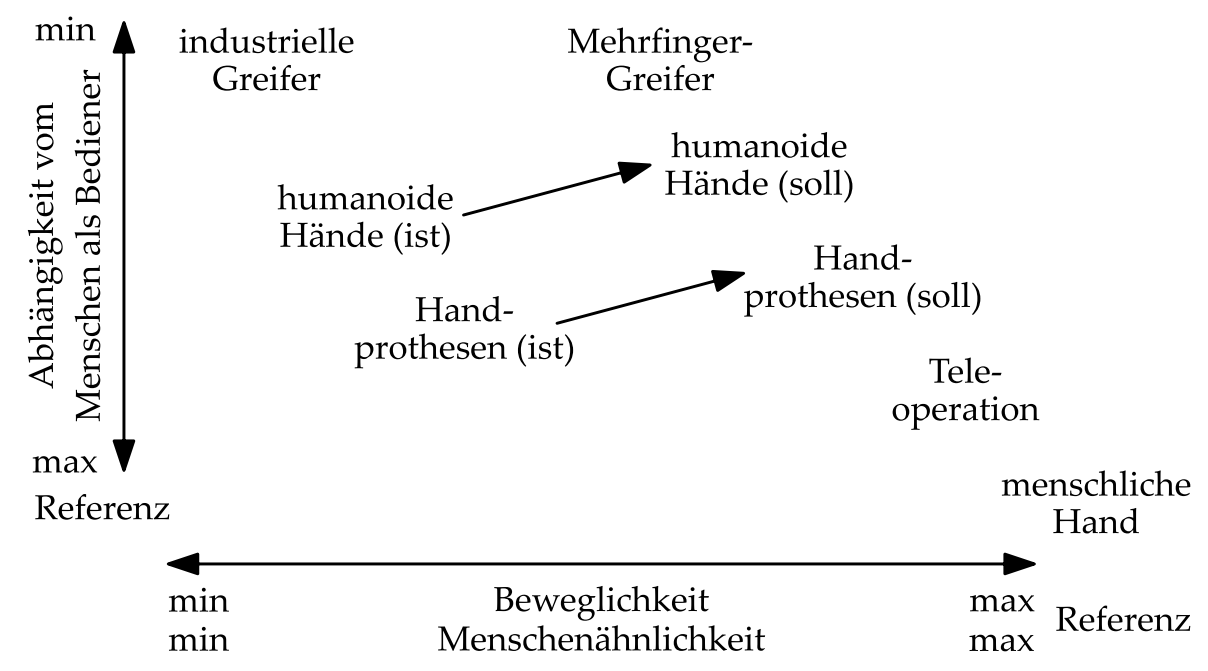

Abbildung 1.4: Einordnung von Händen und Greifern nach ihrer Abhängigkeit vom Menschen und ihrer Beweglichkeit.

Zwischen den beiden zuletzt genannten Fällen sind neue Entwicklungen im Bereich der humanoiden Robotik und der Handprothetik einzuordnen. Mehr Freiheitsgrade und Gelenke als bisher erlauben eine menschenähnlichere Anatomie wie z.B. die Opponierbarkeit des Daumens und unterschiedliche Bewegungsabläufe. Solche Hände erfordern einen höheren Grad der Autonomie von einem Bediener als bisher. Bei Handprothesen ist dies durch Einschränkungen der Ansteuerung mit Hautelektroden begründet. Im Gebiet der humanoiden Roboter reduzieren autonome künstliche Hände den Planungsaufwand für Greifvorgänge. Als weitere Optimierungskriterien folgen Energieverbrauch, Wiederholbarkeit von Aufgaben im menschlichen Umfeld sowie Zuverlässigkeit im regelmäßigen Gebrauch.

\subsubsection{Aktoren}

In künstlichen Händen werden unterschiedliche Antriebs- und Kraftübertragungsprinzipien eingesetzt. Stark verbreitet sind Gleichstrommotoren, die entweder im Unterarm, in der Handfläche oder in den Fingern selbst untergebracht sind. Für die Kraftübertragung zu den Fingergelenken werden meist Seilzüge [60], Spindeltriebe [55, 56], Zahnradgetriebe [112], Harmonic-Drive-Getriebe [22] oder Kombinationen derselben [23] verwendet.

Gemeinsamkeit dieser elektrischen Antriebe ist eine hohe Systemsteifigkeit. Ohne einen Eingriff der Regelung werden bei einem Objektkontakt sehr schnell große Kräfte ausgeübt. Bei der Realisierung der Regelung müssen Effekte wie Getriebespiel oder lastabhängige Dehnung der Seilzüge mit berücksichtigt werden.

In den hydraulisch oder pneumatisch angetriebenen Händen finden sich neben Zylindern mit Kolbenstangen und Hebelmechanik [128], Zylindern mit Bandund Rollenübertragung [37] auch flexible Aktoren, die nach dem Kontraktions- 
prinzip arbeiten [26, 193]. Ein solcher Aktor ist aus einer flexiblen Gummiblase, die von einem Gewebe aus nicht dehnbaren Fäden umschlossen ist, aufgebaut. Eine Erhöhung des Drucks bewirkt eine Querausdehnung der Gummiblase, die durch den äußeren Gewebeschlauch in eine Längskontraktion umgesetzt wird.

In der künstlichen Hand des Forschungszentrums Karlsruhe [172] werden flexible Fluidaktoren nach dem Expansionsprinzip eingesetzt. Diese sehr kompakten Aktoren befinden sich direkt in den Fingergelenken. Sie können beim Einsatz geeigneter Fluide sowohl pneumatisch als auch hydraulisch betrieben werden. Aus der Erhöhung des Drucks im Aktor resultiert eine Längenausdehnung und damit eine Beugung des Gelenks.

Die Finger einer mit solchen flexiblen Antrieben angetriebenen Hand verhalten sich also bei von außen wirkenden Kräften nicht so starr wie die meisten elektrisch angetriebenen Finger. Die Druckerzeugung erfolgt entweder zentral über eine Pumpe, einen Kompressor mit Ventilen oder voneinander getrennte Systeme mit Zylindern, die über Spindeltriebe und Elektromotoren bewegt werden.

\subsubsection{Sensoren}

Erst die vielfältigen Wahrnehmungsmöglichkeiten ermöglichen den Händen des Menschen ihre Geschicklichkeit und Greifsicherheit. Ebenso können sie hierdurch zur aktiven Erkundung der Umwelt eingesetzt werden. Gleiches gilt für künstliche Hände: nur das Wissen über den Zustand der Hand ermöglicht die Regelung ihres Verhaltens und damit die kontrollierte Reaktion auf Umwelteinflüsse [189].

Parallelen zur Physiologie der menschlichen Hand sind hier nur in der Art der gewonnenen Informationen, nicht jedoch in der Weise, wie und wo sie gewonnen werden, zu erkennen. Dies hängt hauptsächlich mit den in künstlichen Händen verwendeten Aktorprinzipien und der Ausführung ihrer mechanischen Struktur zusammen. Auch aus der geplanten Anwendung ergeben sich wichtige Einflüsse auf die Sensorausstattung einer künstlichen Hand. So erfordert die taktile Exploration der Umgebung viel empfindlichere und höher auflösende taktile Sensoren als das sichere Greifen von Objekten.

\section{Winkelpositionen}

Die für die kontrollierte Bewegung der Hand wichtigsten und auch in fast jeder künstlichen Hand vorzufindenden Sensoren sind Positionssensoren zur Messung der Gelenkstellungen. Sie sind die Grundlage für die Regelung der Winkelposition der Gelenke, mit deren Hilfe die Hand in eine definierte Haltung gebracht werden kann. Im naheliegendsten Fall sind diese Winkelsensoren in den Gelenken selbst oder in ihrer unmittelbaren Umgebung angebracht. Als Messprinzipien finden sich Potentiometer [23, 60], optische Sensoren [22] und Magnetfeldsensoren [28, 80, 193].

Befinden sich die Antriebe der Gelenke außerhalb der Finger, so kann die Gelenkwinkelmessung auch hier vorgenommen werden. Vorteilhaft sind die weniger strikten Anforderungen an die Größe der Sensoren und die hohe Auflösung durch die meist erst später im Antriebsstrang wirkende Untersetzung. Dem steht 
als Nachteil entgegen, dass für die Ermittlung der Gelenkposition das Übertragungsverhalten der mechanischen Verbindung zwischen Antrieb und Gelenk genau bekannt sein muss. Eingesetzte Messverfahren sind optische Encoder [51, 55], magnetische Encoder [95, 96] und Linearpotentiometer [128].

\section{Externe Kräfte}

Die Ausstattung mit Sensoren, die Rückmeldung über Kräfte geben, die auf Finger und Handfläche wirken, variiert sehr stark. Bei den auf das Greifen zielenden Projekten finden sich verschiedene Formen von Kraftsensoren, die die an einer oder mehreren Stellen wirkenden Kontaktkräfte bestimmen [108, 109, 139, 196].

Für die taktile Exploration der Umwelt werden Sensoren mit einer großen Anzahl von Messzellen, so genannten Taxels oder Tactels (tactile elements) verwendet, die in einer regelmäßigen Struktur angeordnet sind. Aus den hierbei erfassten Daten kann mittels Analyseverfahren, die meist aus der Bildverarbeitung abgeleitet sind, auf Objektdetails wie z.B. Kanten zurückgeschlossen werden.

\section{Gelenkmomente}

Ergänzend werden bei einigen Händen die in den Gelenken wirkenden Beugemomente bestimmt. Im allgemeinen Fall erfolgt die Messung der Gelenkmomente über Dehnmessstreifen an einem Teil der kraftübertragenden Struktur. Über die bekannte Geometrie und das Materialverhalten wird dann das übertragene Moment berechnet [22].

Je nach Aktorprinzip kann das Gelenkmoment auch aus aktorspezifischen Kenngrößen bestimmt werden: so kann beim Gleichstrommotor aus der Stromaufnahme oder bei einem Zylinder aus dem Fluiddruck über die Kraft und die Geometrie der Mechanik auf das Moment zurückgeschlossen werden. Bei seilzuggetriebenen Händen kann aus der direkt oder indirekt gemessenen Zugkraft der Seilzüge das Gelenkmoment gemessen werden [162]. Die Zuverlässigkeit dieser Ansätze variiert stark, da sie oft von Effekten wie Spiel, Reibung oder Elastizitäten überlagert sind.

Sehr viel aufwändiger sind multiaxiale Kraft-Momenten-Sensoren in den Fingerspitzen [19, 22, 198], die je nach geforderter Genauigkeit aus den Signalen mehrerer Dehnmessstreifen die auf die Fingerspitze wirkenden Kräfte und Momente nach Betrag und Richtung bestimmen können. Mit diesen Informationen kann unter Zuhilfenahme der Fingerspitzengeometrie auch die Lage des Kontaktzentrums ermittelt werden, was die präzise Manipulation von Objekten erleichtert.

\subsubsection{Planung, Steuerung und Regelung}

Die Planung und Synthese von Griffen ist ein der Regelung der Hand übergeordneter Prozess, in dem aus den gegebenen Rahmenbedingungen wie dem zu greifenden Objekt, seiner Lage, seiner Erreichbarkeit und der auszuführenden Aufgabe Zielgrößen für die Handregelung ermittelt werden. 


\section{Greifplanung}

Unterteilt werden die Ansätze hierzu in analytische und wissensbasierte Vorgehensweisen [174]. Analytische Methoden ermitteln aus Modellen und Restriktionen von Objektgeometrie, Handgeometrie, Kontaktbedingungen und Umfelddaten geeignete Kontaktpunkte und -kräfte. Vorteil dieser Methoden ist die Generalisierung der Greifvorgänge und der greifbaren Objekte. Dem steht allerdings ein meist erheblicher Rechenaufwand entgegen. Die generierten Bewegungstrajektorien weisen - wenn überhaupt - nur zufällige Ähnlichkeiten mit Greifvorgängen beim Menschen auf.

Im Gegensatz dazu verwenden wissensbasierte Ansätze A-Priori-Wissen über die zu greifenden Objekte, Regelungsparameter, Umfelddaten sowie Bewegungsund Greifprimitive zur Auswahl einer geeigneten Greifkonfiguration. Diese greifen oft auch auf die menschliche Hand als Vorbild für Greifkonfigurationen zurück und werden deshalb auch physiologische Ansätze genannt [133].

\section{Regelung und Steuerung}

Ein zentrales Regelungskonzept für Roboterhände ist die von Salisbury eingeführte kartesische Steifigkeitsregelung (cartesian stiffness control) [162]. Bei ihr wird mit den Fingerspitzen der Hand ein dem Objektkoordinatensystem achsparalleles Federsystem nachgebildet. So üben die Finger mit steigender Abweichung von ihrer Sollposition auch steigende Kräfte auf das Objekt aus. Hierfür sind in jedem Reglerzyklus die mit den Fingern gemessenen Kräfte in das Objektkoordinatensystem zu transformieren. Aus dem kartesischen Positionsfehler wird über das vorgegebene Federverhalten der Fingerspitze nach einer Rücktransformation das Verhalten der einzelnen Fingergelenke bestimmt.

Hogan erweitert dieses Prinzip zur kartesischen Impedanzregelung (cartesian impedance control) [73] um geschwindigkeitsproportionale Dämpfer. Hierdurch wird direkt auf die Systemdynamik Einfluss genommen. Aus Sicht der Regelungstechnik erfolgt also keine explizite Umschaltung zwischen Positions- und Kraftregelung, stattdessen werden die auszuübenden Kräfte implizit über Sollpositionen und Federkonstanten vorgegeben.

Voraussetzung für die Realisierung dieser Regelungsverfahren in Systemen mit starren Antrieben ist ein hoher Regelungstakt in Verbindung mit einer genauen Positions- und Momentensensorik. Eingesetzt wird sie z.B. in der Karlsruher Hand II [53, 54] oder der DLR-Hand II [22].

\subsection{Offene Probleme}

Heutige Prototypen humanoider Serviceroboter sind nur mit einfachen Greifern oder Händen mit wenigen Aktoren, so genannten underactuated hands ausgestattet. Durch die geringe Anzahl an Freiheitsgraden können solche Hände zwar als Bestandteil des Arms geregelt werden, die Geschicklichkeit im Umgang mit sehr unterschiedlichen Objekten ist dabei begrenzt. Anthropomorphe Hände mit einer 
höheren Zahl regelbarer Freiheitsgrade sind somit ein wichtiger Beitrag zur Weiterentwicklung humanoider Serviceroboter, die in für Menschen geschaffenen unstrukturierten Umgebungen immer wieder unterschiedliche Aufgaben übernehmen und auch mit Menschen interagieren sollen [125].

Ansätze für die mechanische Struktur und den Antrieb solcher Hände werden in [172] vorgestellt. Der Antrieb basiert auf neuartigen flexiblen Fluidaktoren, die die Konstruktion leichter Hände mit nachgiebigen Gelenken ermöglichen. Mit ihnen können bereits durch eine Ablaufsteuerung Greifvorgänge ausgeführt werden. Das wird am Einsatz in einer Handprothese demonstriert. Für dieses Handkonzept ist bisher offen, wie das Greifen weiter automatisiert und die Greifsicherheit und Geschicklichkeit erhöht werden kann.

Als offene Probleme sind zu nennen:

- Wie verhalten sich pneumatisch und hydraulisch angetriebene flexible Fluidaktoren in den Fingergelenken einer künstlichen Hand und welche Auswirkungen hat dies auf das Greifen?

- Welche Sensorklassen können in Verbindung mit einer Steuerung oder Regelung für die Erhöhung der Greifsicherheit vorteilhaft eingesetzt werden?

- Welche Sensoren sind für die gegebene Ausführung der Hand einsetzbar, ohne ihre anthropomorphe Gestalt nachteilig zu verändern?

- Welche Verfahren sind für die Regelung der Gelenke geeignet und wie kann das nachgiebige Verhalten der Aktoren vorteilhaft ausgenutzt werden?

- Mit welchen Steuerungsmethoden kann die Geschicklichkeit und Menschenähnlichkeit der Handbewegungen verbessert werden?

- Wie kann die Hand autonom auf die Veränderung der Umgebung reagieren?

\subsection{Ziele und Aufgaben}

Das Ziel dieser Arbeit besteht darin, Grundlagen für die Weiterentwicklung der Greiffähigkeiten einer von flexiblen Fluidaktoren angetriebenen anthropomorphen Hand für einen humanoiden Roboter zu schaffen. Dies ist in die Themen Modellierung, Sensorik, Regelung und Greifsteuerung aufzuteilen.

Im zweiten Kapitel werden aus der Analyse der Physiologie, der Aktionen und der Fähigkeiten der menschlichen Hand die Aufgaben und Fähigkeiten einer humanoiden Roboterhand abgeleitet sowie die sich daraus ergebenden Anforderungen an das Sensorsystem und die Steuerung beschrieben.

Das dritte Kapitel umfasst die Herleitung von mathematischen Modellen aus der Vermessung der Aktoren zur Beschreibung des Momenten-, Volumen- und Steifigkeitsverhaltens flexibler Fluidaktoren im pneumatischen und hydraulischen Betrieb. 
Als Grundlage für die Regelung und Überwachung der Roboterhand werden im vierten Kapitel verschiedene Sensorprinzipien dargestellt und ihre Einsatzmöglichkeiten in einer von flexiblen Fluidaktoren angetriebenen Hand bewertet und ausgewählt. Für die ausgewählten Sensoren werden anschließend Integrationslösungen erarbeitet, Varianten verglichen, Optimierungen hergeleitet, und ihre Funktion am Prototypen nachgewiesen. Diskutiert werden die Vor- und Nachteile unterschiedlicher Materialien und Strukturen der Fingerverkabelung.

Im fünften Kapitel wird der Einsatz der Sensoren bei der Gelenkregelung im pneumatischen und hydraulischen Betrieb demonstriert. Hierzu wird das Verhalten des Gelenks und weiterer Aggregate wie z.B. Pumpe und Ventile als Modelle beschrieben. Aufgezeigt wird, wie sich das nachgiebige Verhalten der Aktoren für die Greifsicherheit ausnutzen lässt und wie sich die Interaktion mit Objekten durch eine gezielte Momentenbegrenzung des Positionsreglers verbessern lässt. Die Steuerung von Greifvorgängen, die Koordination von Arm- und Handbewegungen sowie die Struktur einer Handsteuerung werden dargestellt.

Das sechste Kapitel fasst die Ergebnisse der Arbeit zusammen, gibt einen Ausblick auf die Verwendung der Sensoren in Händen für einen humanoiden Roboter und zeigt weitere Entwicklungsmöglichkeiten auf. 


\section{Anforderungen an humanoide Roboterhände}

Die Entwicklung von Händen für einen humanoiden Roboter stellt neue Herausforderungen, da die in der Forschung verbreiteten mehrfingrigen Robotergreifer meist nicht anthropomorph gestaltet sind und ihr Greifverhalten das des Menschen nur unbefriedigend abbildet.

In diesem Kapitel erfolgt nach einer Darstellung der Anatomie und Physiologie der menschlichen Hand eine Klassifikation und Ablaufbeschreibung menschlicher Greifvorgänge sowie eine Auswahl hiervon für den Einsatz in einem humanoiden Roboter.

Diese Tätigkeiten werden den verschiedenen Rezeptoren zugeordnet und daraus Anhaltspunkte für die Sensorausstattung künstlicher Hände in der humanoiden Robotik abgeleitet. Unter Berücksichtigung der gewählten Sensoren werden abschließend die Anforderungen an die Steuerungs- und Regelungsmechanismen einer solchen Hand beschrieben.

\subsection{Physiologie der menschlichen Hand}

In diesem Abschnitt wird der Aufbau der menschlichen Hand hinsichtlich Anatomie, Biomechanik, Gewebe, Haut und Wahrnehmung analysiert. Aus diesen Erkenntnissen werden später Anhaltspunkte für die Auswahl und Auslegung der Sensoren einer künstlichen Hand abgeleitet.

\subsubsection{Anatomie und Biomechanik}

Die Grundstruktur der menschlichen Hand ist ein fünffingriges Skelett, das über Sehnen von einen extrinsischen Muskelapparat im Unterarm bewegt wird [89]. Insgesamt besteht das Skelett aus 27 Knochen: Acht davon bilden das Handgelenk (Carpus), die Finger werden aus jeweils vier Knochen gebildet, der Daumen bildet mit nur drei Knochen eine Ausnahme. Die Mittelhandknochen (Metacarpus) befinden sich im Bereich der Handfläche, hieran schließen sich die Fingergrundglieder (proximale Phalanx), die Mittel- (mittlere Phalanx) und die Endglieder (distale Phalanx) an. Die drei Fingergelenke (MCP- metacarpophalangeal, PIP-proximal interphalangeal, DIP- distal interphalangeal) erlauben die Beugung der Finger zur Handinnenfläche, das Fingergrundgelenk eine zusätzliche seitliche Bewegung (Abduktion und Adduktion). 


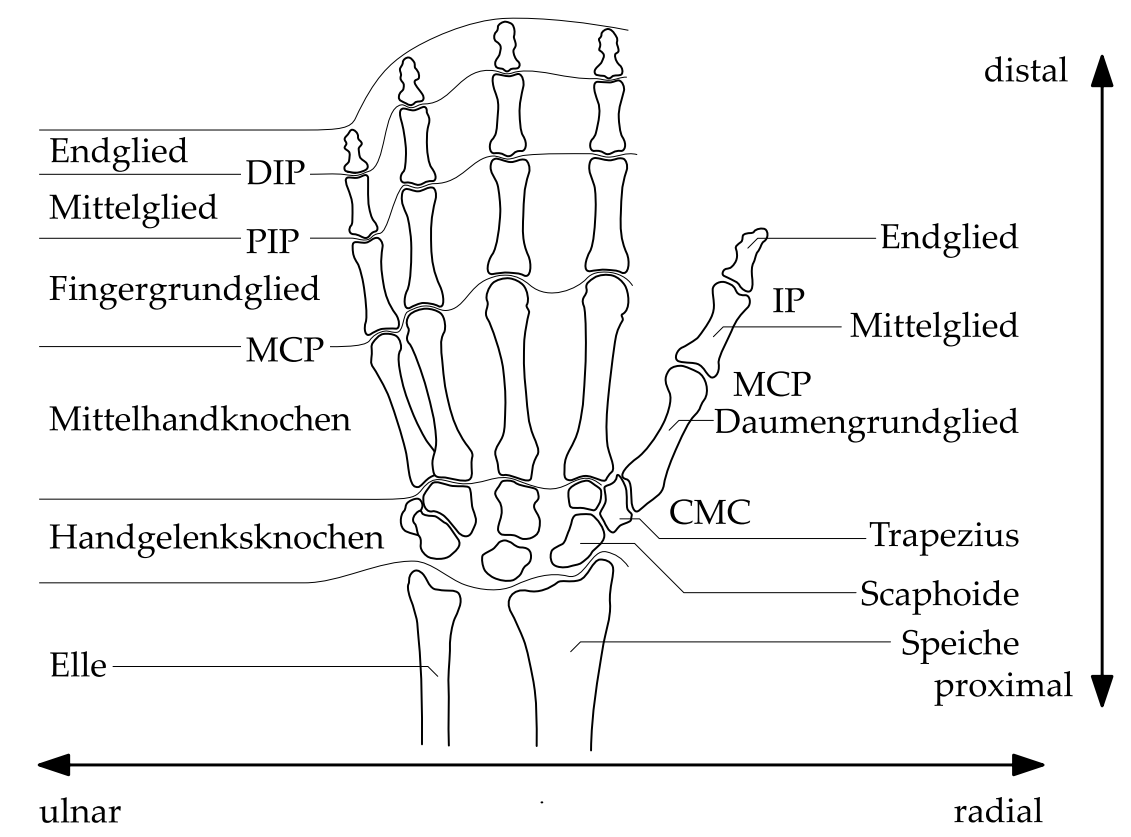

Abbildung 2.1: Benennung der Knochen und Gelenke der menschlichen Hand nach [137].

Der Besonderheit des Daumens ist sein Sattelgelenk an der Handwurzel (CMC-carpo-metacarpal), das die Opposition (Gegenüberstellung) zu den anderen Fingern möglich macht. Hierdurch können einerseits mit den Fingerspitzen kleine Gegenstände präzise gegriffen werden wie auch große Objekte sicher umschlossen werden.

Die Flexion (Beugung) und die Extension (Streckung) der Gelenke erfolgt antagonistisch, also mittels einander entgegengesetzt als Beuger und Strecker wirkenden Sehnen und Muskeln [188]. Dabei ist der Sehnenapparat für die Flexion deutlich aufwändiger und kräftiger als der für die Extension. Außerdem ergibt sich durch die Struktur des Sehnenapparats eine teilweise Kopplung der Bewegungen mehrerer Finger (Abbildung 2.2). Insbesondere die distalen Gelenke lassen sich nicht beliebig unabhängig von den proximalen bewegen.

\subsubsection{Gewebe und Haut}

Zum sicheren Greifen der menschlichen Hand leisten die Haut und das darunter liegende Gewebe einen wichtigen Beitrag, indem sie unabhängig von der Elastizität des gegriffenen Objekts auch bei geringen Greifkräften einen flächigen Kontakt herstellen.

Haut und Gewebe der Fingerspitze bilden vier Lagen (Abbildung 2.3): an der Hautoberfläche befindet sich eine nur wenige Zellen dicke Hornhaut, sie besteht aus abgestorbenen Zellen der darunter liegenden Epidermis. Corium und Subcutis stellen das nachgiebige Bindegewebe dar, das Verbindung zu den Knochen 


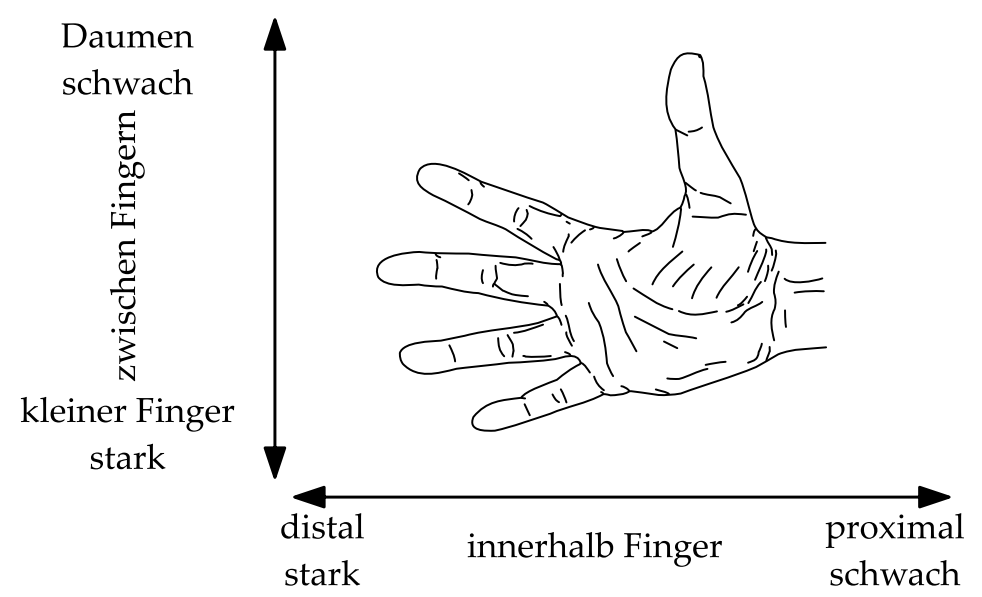

Abbildung 2.2: Qualitative Kopplung der Freiheitsgrade in den Fingern der menschlichen Hand: Zunahme der Kopplung von proximal nach distal und von Daumen zum kleinen Finger nach [89].

schafft [150]. Aus der Nachgiebigkeit dieser beiden Schichten ergibt sich beim Greifen eines Objekts schnell eine große Kontaktfläche. Hierdurch wird die Lage des Objekts in der Hand schneller stabilisiert als mit einer harten, glatten Oberfläche. Gleichzeitig verteilt sich die resultierende Kontaktkraft auf eine größere Fläche. Dadurch wird die Flächenpressung und damit auch das Risiko der Verletzung der Hand oder der Beschädigung eines gegriffenen Objekts reduziert.

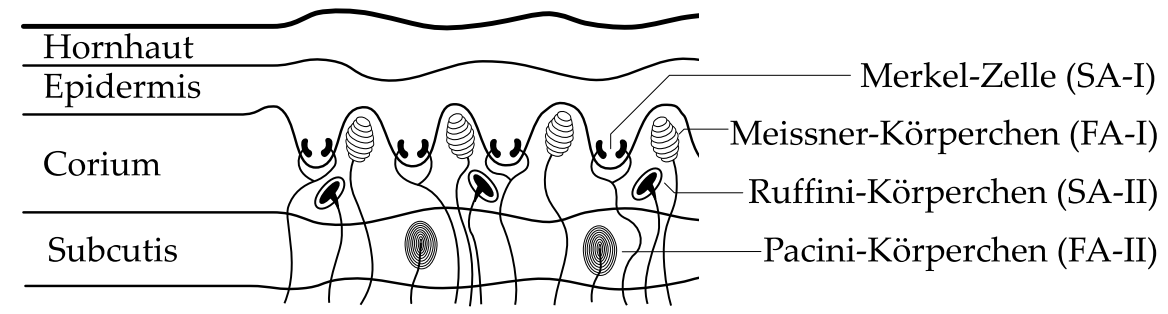

Abbildung 2.3: Schematischer Schichtenaufbau und Lage der Rezeptoren in der unbehaarten Haut nach [164].

\subsubsection{Wahrnehmung}

Neben ihrer Funktion als Werkzeug ist die Hand eines der wichtigen Sinnesorgane des Menschen. Ihre Wahrnehmung wird aus Sicht der Physiologie in die Außen- und die Eigenwahrnehmung sowie die Schmerzwahrnehmung aufgeteilt [134, 164].

Die haptische Wahrnehmung beschreibt die Integration der teilweise redundan- 
ten Informationen der Außen- und die Eigenwahrnehmung [106, 115], also z.B. die Feststellung von Größe, Form und Gewicht eines gegriffenen Objekts.

\section{Außenwahrnehmung}

Die Außenwahrnehmung (Exterozeption, extrinsische Wahrnehmung) ist die direkte taktile Wahrnehmung der Umwelt durch die Mechanorezeptoren der Haut. Mit ihnen nimmt der Mensch bei Berührung und Griffkontakt viele Umwelteigenschaften wie Oberflächenstruktur, Elastizität, Temperatur oder Wärmeleitfähigkeit wahr [106].

Die Mechanorezeptoren in der Haut sprechen auf unterschiedliche mechanische Reize bei der Verformung der Haut und des darunter liegenden Gewebes an. Sie sind im Bindegewebe und der unteren Epidermis zu finden (Abbildung 2.3) und werden nach ihrer Reaktionsgeschwindigkeit und Empfindlichkeit in vier Gruppen klassifiziert:

Merkel-Zellen (SA-I, slowly-adapting I) sind in der unteren Epidermis zu finden und geben die Intensität der Hautverformung wieder. Sie sind damit für die Bestimmung von dauerhaft wirkenden Kräften besonders wichtig.

Meissner-Körperchen (FA-I, fast-adapting I) finden sich in der äußeren Schicht des Corium und reagieren auf die Geschwindigkeit mechanischer Reize.

Ruffini-Körperchen (SA-II, slowly-adapting II) liegen tiefer als die MerkelZellen im Corium und werden ähnlich wie diese durch die Intensität statischer Belastungen angeregt.

Pacini-Körperchen (FA-II, fast-adapting II) in der unteren Subcutis detektieren Beschleunigung und Schwingungen des Gewebes.

Neben der Zunge und den Lippen ist die Dichte dieser Rezeptoren auf der Handinnenfläche am höchsten. In diesen Bereichen kann der Mensch Reizpunkte mit Abständen von nur einem Millimeter erkennen. Die Auflösung nimmt zusammen mit den SA-I- und den FA-I- Rezeptoren von den Fingerspitzen mit 1,7 mm auf etwa $8 \mathrm{~mm}$ zur Handfläche ab. Die Dichte der SA-II- und FA-II- Rezeptoren bleibt dagegen annähernd konstant. In der behaarten Haut sind statt der MeissnerKörperchen Haarfollikel-Sensoren an den Haarwurzeln zu finden, über die die Bewegung der Körperbehaarung wahrgenommen wird.

\section{Eigenwahrnehmung}

Die Eigenwahrnehmung (Propriozeption, intrinsische oder auch kinästhetische Wahrnehmung) ist die Wahrnehmung des inneren Zustands der Hand wie z.B. Stellung der Fingergelenke, auf sie wirkende Kräfte oder Anspannung der Sehnen in der Hand und der Muskeln im Unterarm [105].

Die drei Typen von Mechanorezeptoren in den Gelenken und ihrer direkten Umgebung sind ähnlich denen in der Haut aufgebaut: Einige Ruffini-Körperchen 
(SA-II) sind in den Fasern der Gelenkkapseln zu finden. Sie ermitteln Gelenkwinkel und -bewegungen. Pacini-Körperchen (FA-II) erfassen in den Muskelansätzen Beschleunigungen und Verzögerungen. Die Extremlagen der Gelenke werden von den Golgi-Sehnenorganen erkannt, die den Ruffini-Körperchen ähneln und sich in den Sehnen und den Übergängen zur Muskulatur befinden. Ihre Rückmeldung wird der Schmerzwahrnehmung zugerechnet.

Aus der Muskulatur liefern zwei Typen von dehnungsempfindlichen Rezeptoren, die Muskelspindeln und die Golgi-Sehnenorgane, eine Rückmeldung über die Kontraktion der Muskeln.

\subsection{Aktionen der menschlichen Hand}

Die Tätigkeiten der menschlichen Hand lassen sich in die vier Hauptgruppen unterteilen: Gesten, Haltungen, Greifen und Manipulation. Sie werden in den folgenden Abschnitten definiert und es werden ihre Abläufe analysiert.

\subsubsection{Definitionen}

\section{Gesten}

Unter den Gesten werden alle Handkonfigurationen und Bewegungsabläufe ohne eine beabsichtigte Interaktion mit einem Objekt zusammengefasst. Dies umfasst nicht nur die eigentlichen Gesten mit einer entsprechenden Bedeutung, sondern auch die in der Physiologie Formierung [164] oder in der Robotik Pregrasp [88] genannten Vorbereitung der Hand auf einen folgenden Objektkontakt. In dieser Phase wird z.B. der Daumen in Opposition zu Zeige- und Mittelfinger gebracht, um anschließend eine Flasche zu umgreifen. Auch das Anfahren einer entspannten Referenzposition gehört zu dieser Gruppe.

\section{Haltungen}

Der Gruppe der Haltungen sind alle Handaktivitäten zugeordnet, bei denen Kontakt mit einem Objekt besteht, ohne dieses fest zu umschließen. Bewegungen der Fingergelenke resultieren aus der Interaktion mit Objekten, gegenüber diesen weist die Hand ein vorgegebenes Kräfteverhalten auf.

Beispiele für Haltungen sind das Balancieren eines Tabletts auf der offenen Hand, das Betätigen eines Schalters mit der Spitze des Zeigefingers und das Öffnen einer Schublade mit einem Hakengriff. Ein Sonderfall ist die taktile Exploration, bei der mit aktiven Bewegungen von Arm und Fingern Informationen über Objekteigenschaften wie Geometrie und Oberfläche gewonnen werden.

\section{Greifen}

Die Zielsetzung des statischen Greifens ist es, ein Objekt in einer stabilen Lage relativ zur Hand zu halten. Ein Verrutschen oder Fallenlassen auch unter der Einwirkung externer Kräfte wird als unerwünscht angesehen. 
Bei Greifvorgängen werden Veränderungen von Position und Ausrichtung des gegriffenen Objekts im Raum durch Armbewegungen realisiert. Diese so genannte Arm-Manipulation ist heute sowohl in der industriellen Robotik als auch in der Handprothetik weit verbreitet. Sichere Greifvorgänge werden durch große Kontaktflächen und ein weitgehendes Umschließen des Objekts erreicht.

\section{Manipulation}

Im Gegensatz zum Greifen ist die Manipulation von Objekten mit den Fingern die kontrollierte Änderung von Position und Ausrichtung eines Objekts in der Hand [20]. Sie wird durch die gezielte Ausübung von Kräften und Momenten auf das gegriffene Objekt und die Veränderung der Fingerkonfiguration erreicht.

So stellt als Beispiel das Eindrehen einer Glühlampe in eine Fassung durch koordinierte Abduktions- und Adduktionsbewegungen der Finger einer feststehenden Hand an diese deutlich höhere Ansprüche als der gleiche Vorgang mit einem statischen Griff und der Ausführung der Rotation durch Einwärts- und Auswärtsdrehen des Unterarms (Pronation und Supination).

Ein Manipulationsvorgang hat als Ausgang und Ziel geeignete Handkonfigurationen, die sowohl das Objekt sicher gegriffen halten, der Hand jedoch immer noch genug Freiheitsgrade für Bewegungen geben.

\subsubsection{Ablauf von Handaktionen}

Die im vorangegangenen Abschnitt definierten Aktionen der menschlichen Hand sind als voneinander abhängige Vorgänge zu betrachten [88]. Eine Reihe möglicher Abfolgen und der ausgeübten Kräfte sind in Abbildung 2.4 dargestellt.

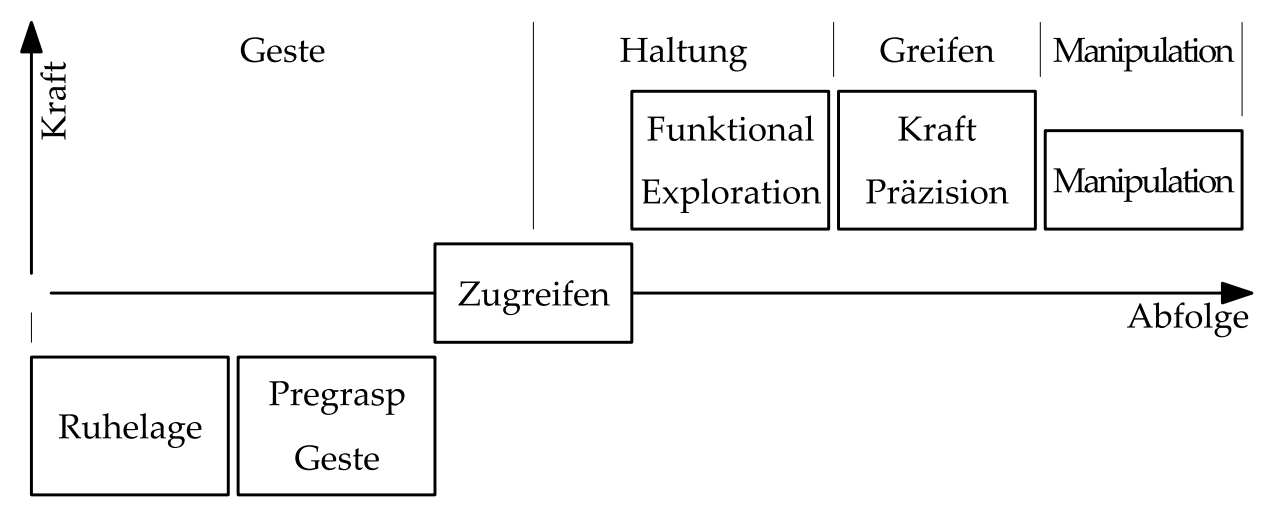

Abbildung 2.4: Ablauf von Greif- und Manipulationsvorgängen.

\section{Ruhelage, Pregrasp und Gesten}

Ausgehend von einer Ruhelage führt die Hand ohne Objektkontakt eine freie Bewegung aus. Sie ist meist mit einer Armbewegung zur Positionierung der Hand 
im Raum verbunden. Ein Objektkontakt ist noch nicht erwünscht und kann als Fehlerfall behandelt werden.

Aus technischer Sicht liegt eine ungehinderte Bewegung vor. Ein Kontakt mit Objekten wird als Fehlerfall betrachtet. Gesten werden mit Hilfe einer Positionsregelung der Gelenke und entweder statischen oder zeitlich veränderlichen Führungsgrößen realisiert.

\section{Zugreifen}

In dieser Phase erfolgt erst der Übergang in eine Haltung, in der bereits Objektkontakt besteht, das zu greifende Objekt aber noch nicht sicher umschlossen ist. Sukzessive werden die Finger an die erforderlichen Kontaktpunkte herangefahren. Um ein Verschieben oder Umfallen des zu greifenden Objekts zu verhindern, dürfen nur kleine Kräfte ausgeübt werden.

\section{Greifen}

Nach der Haltung erfolgt der Übergang zum Greifen fließend, bis an allen erforderlichen Kontaktpunkten das geforderte Kraftniveau erreicht ist. Bedingung für das Greifen ist, dass sich die Lage des Objekts in der Hand unter den gegebenen Randbedingungen (z.B. Beschleunigung durch Anheben) nicht mehr ändert. Eine Lageänderung des Objekts in der Hand wird beim Greifen als Fehler- oder Versagensfall angesehen. Der eigentliche Greifvorgang ist also als Kraftregelung darstellbar.

\section{Manipulation}

Als weiterer Schritt nach dem Greifen kann sich eine Manipulationsphase anschließen. Während dieser wird durch eine gezielte Änderung des Griffmusters die Lage des Objekts in der Hand kontrolliert verändert. Abhängig von Faktoren wie Handkinematik, Greifmuster, Größe und Gewicht des Objekts kann eine Manipulationsbewegung aber auch unmöglich werden, da z.B. die Stabilität des Griffs nicht mehr gegeben ist. In jedem Falle erfordert diese Tätigkeit eine gleichzeitige Kontrolle von Kraft und Bewegung aller Finger und stellt damit deutlich höhere Anforderungen als ein Greifvorgang.

\subsubsection{Klassifikation von Greifmustern}

Die menschliche Hand kann durch ihre vielen Freiheitsgrade eine große Anzahl unterschiedlicher Greifhaltungen annehmen. Die Klassifikationsschemata in der Physiologie [89] orientieren sich meist nur an der Erscheinung, bestenfalls erfolgt eine Einordnung in Kraft- und Präzisionsgriffe [136]: Mit Kraftgriffen werden Objekte sicher mit einer möglichst großen Kontaktfläche, die auch die Handfläche einschließt und hohen Kräften umgriffen. Dagegen sind die Kontaktflächen bei Präzisionsgriffen sehr viel kleiner. Sie beschränken sich meist auf die Fingerspitzen. Dies erlaubt eine größere Geschicklichkeit und Feinfühligkeit im Umgang mit Objekten sowie die gezielte Manipulation der Objektlage in der Hand. 
Neuere Schemata (Abbildung 2.5) erweitern diesen Ansatz zu einer Grifftaxonomie für Produktionsumgebungen, die Kraft- und Präzisionsgriffe nach Objektgeometrie um verschiedene Untergruppen wie sphärische und zylindrische Griffe erweitert. Außerdem wird die hier als Haltung beschriebene Klasse der 'nicht umgreifenden Griffe' (non-prehensile grasps) eingeführt.

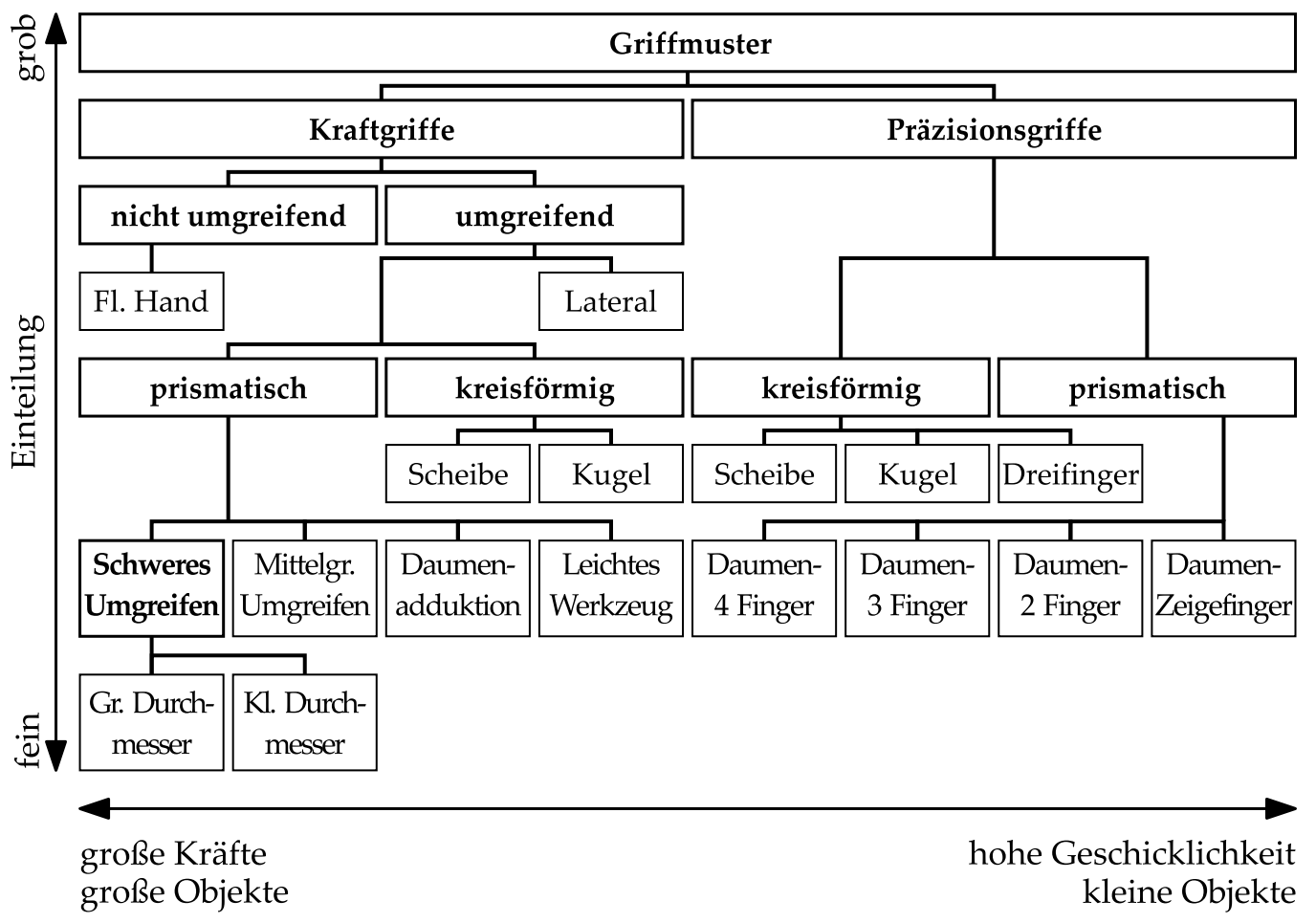

Abbildung 2.5: Grifftaxonomie für Produktionsumgebungen nach [34, 35].

\subsubsection{Programmieren durch Vormachen}

Weitere Ansätze zur Klassifikation von Handaktionen kommen aus dem Programmieren durch Vormachen (programming by demonstration). Hier wird aus der Demonstration eines Ablaufes durch automatisierte Zerlegung eine verallgemeinerte Form der aufeinander folgenden Arbeitsschritte abgeleitet [45, 57, 157]. Diese kann dann auf ähnliche Situationen übertragen und von einem Roboter wieder ausgeführt werden. Die Details von Greifvorgängen werden dabei meist von Datenhandschuhen mit zusätzlichen Sensoren zur Messung der Kontaktkräfte aufgenommen [204]. Die Erkennung von Objekten erfolgt über Stereokameras, die Position der Hand wird von zusätzlichen 3D-Trackingsensoren erfasst.

Für die Erkennung von Greifmustern werden Methoden wie das Kontaktnetz (contact web) verwendet [88]. Aus der Geometrie des Verbindungsnetzes aller Kontaktpunkte zwischen Objekt und Hand wird dabei auf das Greifmuster geschlossen. Unterschieden wird dabei zuerst an Hand der Tatsache, ob Griffe die Handflä- 
che einbeziehen (volar grasps) oder nicht (non-volar grasps). Als zweites Kriterium dient bei letzteren die Planarität des Kontaktnetzes.

Ein weiterer Teilaspekt des Programmierens durch Vormachen ist die Übertragung der gewonnenen Arbeitsschritte von der menschlichen Hand auf nichtanthropomorphe Greifergeometrien. In etwas veränderter Form ist diese Aufgabe auch bei der Teleoperation zu lösen. Die Klasse der Gesten als Kommunikationsform findet bisher in keines dieser Schemata Eingang.

\subsection{Einzelanforderungen}

\subsubsection{Aufgaben und Fähigkeiten}

Die Fähigkeiten eines humanoiden Roboters können durch Hände mit mehr unabhängigen Freiheitsgraden merklich erweitert werden. Dies ist mit einem deutlich erhöhten Aufwand zur Steuerung und Regelung verbunden. Zur Vereinfachung von Greifaufgaben wird daher eine zusätzliche Systemebene, die teilautonom die Ausführung von Greifaufgaben übernimmt, eingesetzt. So kann eine übergeordnete Planungsebene auf die Ermittlung eines geeigneten Greifmusters mit den dazugehörigen Objektparametern beschränkt werden. Während der Durchführung der geplanten Aktion erfolgt eine Synchronisation der Arm- und Handaktivitäten nur zu einzelnen Zeitpunkten.

In Anlehnung an die bisher behandelten analyseorientierten Greifklassifikationen wird eine neue, ausführungsorientierte Aufgabenklassifikation (Abbildung 2.6) für humanoide Roboter notwendig [146]. Die Einordnung erfolgt an den bereits eingeführten Aufgabentypen Gesten, Haltung und Greifen, wobei hier wie bei der Klassifikation in Abbildung 2.5 auch zwischen Kraft- und Präzisionsgriffen unterschieden wird.

\begin{tabular}{|c|c|c|c|}
\hline \multicolumn{4}{|c|}{ Griffmuster } \\
\hline Gesten & Haltungen & Kraft & Präzision \\
\hline Kein Objektkontakt & $\begin{array}{c}\text { Objektkontakt, } \\
\text { nicht umgreifend }\end{array}$ & $\begin{array}{c}\text { Große } \\
\text { Kontaktfläche }\end{array}$ & $\begin{array}{c}\text { Fingerspitzen- } \\
\text { Kontakt }\end{array}$ \\
\hline Referenz & Taster/Schalter & Prismatisch* & Prismatisch ${ }^{*}$ \\
\hline Zeigen & Schieben & Sphärisch* & Kreisförmig* \\
\hline Pregrasp & Haken & Lateral & Dreifinger* \\
\hline
\end{tabular}

Abbildung 2.6: Grifftaxonomie für einen humanoiden Roboter, mit * markierte Griffmuster sind in unterschiedlichen Größen zu realisieren [146]. 
Die Griffmuster in Abbildung 2.5 werden in diskreten Größen unterschieden. Für einen humanoiden Roboter sind diese zu einem Muster zusammengefasst, das mit unterschiedlichen Objektgrößen versehen werden kann. Damit ist auch implizit definiert, wie sich eine Hand beim Zugreifen weiterbewegen kann, wenn der erwartete Objektkontakt nicht eintritt. So kann beispielsweise auch mit der Vorgabe eines zylindrischen Objekts ein konisches Glas gegriffen werden, ohne hierfür ein exaktes Greifverhalten vorzugeben.

Die Auswahl der Greifmuster in der Taxonomie für einen humanoiden Roboter ist aus alltäglichen Aktivitäten in einem Haushaltsumfeld abgeleitet. Diese umfassen im Einzelnen:

- Greifen von aufrecht stehenden mittelgroßen und großen Objekten (z.B. Gläser, Tassen und Flaschen) von der Seite und von oben,

- Greifen von kleinen, liegenden Objekten (z.B. Stifte und Besteck) von oben,

- Betätigung von Tasten, Druck- und Drehschaltern,

- Öffnen von Türen und Schubladen,

- Schiebebewegungen (z.B. Schließen einer Schublade, Objekte auf einer Arbeitsfläche) sowie

- einfache Gesten (z.B. Zeigebewegungen).

Auf die Realisierung weiter gehender Fähigkeiten wie z.B. der Fingermanipulation wird hier verzichtet, da sich solche Operationen zumindest teilweise auch auf die Arm-Manipulation übertragen lassen. Ebenso sollen keine spezifischen Fähigkeiten für die taktile Exploration implementiert werden.

\subsubsection{Handkinematik}

Aus der Analyse der menschlichen Hand und den geforderten Fähigkeiten einer humanoiden Roboterhand sind auch Anhaltspunkte für Kinematik und Aktoranordnung abzuleiten. Zuallererst ergibt sich aus der Zielstellung einer möglichst menschenähnlich erscheinenden Hand ein fünffingriges Design mit einem opponierbaren Daumen.

Im Unterschied zu den underactuated hands mit sehr wenigen Aktoren und einer meist elastischen Kopplung der Freiheitsgrade sind möglichst wenige der Freiheitsgrade miteinander zu koppeln. Besonders wichtig ist die Entkopplung des Daumens, da seine Positionierung in Relation zu den anderen Fingern sehr präzise erfolgen muss. Eine Kopplung von Freiheitsgraden ist bei Ringfinger und kleinem Finger am ehesten zulässig, da sie bei Greifvorgängen oft nur unterstützend wirken [120].

Aus der vorgestellten Grifftaxonomie ergeben sich eine Reihe weiterer notwendiger Entkopplungen: Für die Betätigung von Tastern und Zeigebewegungen muss es möglich sein, den Zeigefinger unabhängig von den anderen Fingern gestreckt zu lassen. Dreifingergriffe erfordern eine Entkopplung des Ringfingers und des kleinen Fingers von Zeige- und Mittelfinger. 
Wird die Flexion der Finger wie beim Menschen mit drei Freiheitsgraden ausgeführt, so dürfen am ehesten die beiden distalen Gelenke miteinander gekoppelt werden. Sollen weitere Freiheitsgrade eingespart werden, so kann zuerst auf die distalen Aktoren in den Gelenken verzichtet werden.

Die Verbesserung der Manipulationsfähigkeit erfordert die Realisierung einer unabhängigen Abduktion und Adduktion der Finger [162]. Für die weitere Diskussion wird von einer Hand mit 11 unabhängigen Freiheitsgraden ausgegangen, zwei Freiheitsgrade je Finger dienen dabei der Flexion, der Daumen ist mit einem zusätzlichen Gelenk für die Oppositionsbewegung ausgestattet.

\subsubsection{Auswahl der Sensorklassen}

Für den Einsatz in einem humanoiden Roboter sollen, in Anbetracht der möglichst menschenähnlichen Gestaltung, externe Sensoren wie z.B. zur Objekterkennung verwendbare Näherungssensoren, Laserscanner oder Kameras in Handteller und Umgebung vermieden werden. Verzichtet werden soll ebenso auf einen differentiell wirkenden Tastsinn, mit dem beispielsweise raue Oberflächen abgetastet werden können oder das Verrutschen eines Objekts in der Hand durch Vibrationen erkannt werden kann.

In unstrukturierten Umgebungen können einem humanoiden Roboter Planungsfehler unterlaufen, die zu unerwünschten Kollisionen der Hand mit der Umgebung führen. Zur Vermeidung von Schäden oder gar der Verletzung von Menschen muss der Handsteuerung die Gelegenheit gegeben werden, solche Situationen zu erkennen. Dies sind insbesondere das Erreichen der Gelenkextremlagen über den Arbeitsbereich des Aktors hinaus, das Überschreiten der von den Aktoren maximal ausübbaren Kontaktkräfte z.B. durch Einquetschen der Finger und das Erreichen des maximalen Aktordrucks durch von außen wirkende Kräfte. Das entspricht aus Sicht eines humanoiden Roboters der Schmerzwahrnehmung und den damit verbundenen Reflexen.

Somit verbleiben drei Sensorklassen, für die geeignete Messprinzipien zu finden sind:

- Winkelsensoren zur Erfassung der Gelenkpositionen,

- Flächige taktile Kraftsensoren zur Bestimmung der Kontaktkräfte insbesondere an den Fingerinnenseiten sowie

- Momentensensoren für die Messung der in den angetriebenen Gelenken übertragenen Drehmomente.

\subsubsection{Anforderungen an die Sensoren}

Alle zu entwickelnden Sensoren haben eine Reihe typenunabhängiger Anforderungen zu erfüllen. Diese sind:

Einfachheit: Die Sensoren sind möglichst einfach und preiswert auszuführen, auch auf Kosten ihrer Messgenauigkeit. 
Kompatibilität zu Aktoren und Mechanik: Die Sensoren sollen sich Eigenschaften der Aktoren, mit denen sie zusammen eingesetzt werden, zu Nutze machen. Die Mechanik ist so wenig wie möglich zu verändern.

Größe: Die Sensoren für den Einsatz in den Fingern sind so kompakt wie möglich auszuführen und dürfen die Außenmaße der Finger nicht verändern.

Gewicht: Die Sensoren dürfen das Gewicht der sensorlosen Finger von etwa $40 \mathrm{~g}$ nicht wesentlich erhöhen.

Räumliche Anordnung: Die Sensoren sind möglichst direkt am Messort anzubringen. Die zu messende Größe soll nicht durch mechanische Übertragungsstrecken verfälscht werden. Kann eine bereits vorhandene, in ihrem Verhalten gut beschreibbare Übertragungsstrecke genutzt werden und dadurch ein Sensor aus den Fingern ausgelagert werden, so ist diese Variante zu wählen.

Absolute Messung: Die Sensoren sollen absolute Signale liefern. Dies erlaubt eine einfache Ausführung der Datenerfassung.

Kalibrierung: Die Sensoren sollen nach einer einmaligen Kalibrierung sicher wiederholbare Ausgangssignale liefern. Eine wiederholte Kalibrierung soll nicht erforderlich sein.

Elektrische Schnittstelle: Die Sensorsignale sind so aufzubereiten, dass sie direkt von einem A/D-Wandler aufgenommen werden können. Die Sensoren sollen keine besondere Initialisierung benötigen. Eine Deaktivierung zur Energieersparnis soll möglich sein.

Temperaturbereiche: Solange Prototypen des humanoiden Roboters nur unter Laborbedingungen mit Raumtemperatur und moderaten Abweichungen davon betrieben werden, ist ein zulässiger Temperaturbereich von $\left(23 \pm 5^{\circ} \mathrm{C}\right)$ ausreichend. 


\section{Flexible Fluidaktoren}

Neben den in der Industrie weit verbreiteten starren Fluidaktoren in Form von Zylindern unterschiedlicher Bauform werden auch flexible Fluidaktoren in Form von elastischen Kammern eingesetzt. Gemeinsam ist all diesen Aktoren die Ausübung einer Kraft und die Ausführung einer Bewegung entlang der Arbeitsrichtung bei Beaufschlagung mit einem Fluid, also einem Gas oder einer Flüssigkeit. Bei Zylindern wird dies durch einen im Zylinderkörper beweglich gelagerten Stempel realisiert. Flexible Fluidaktoren bestehen dagegen aus einer elastischen, geschlossenen Kammer, die in Abhängigkeit vom Innendruck ihre Form ändert.

Hydraulik- und Pneumatikzylinder sind an die Anforderungen des jeweiligen Mediums angepasst. Dies betrifft insbesondere Festigkeit und Dichtungen für die unterschiedlichen Druckbereiche und die damit verbundenen Aktorkräfte. Flexible Fluidaktoren können durch ihre geschlossene Bauweise sowohl pneumatisch als auch hydraulisch betrieben werden. Voraussetzung ist jedoch die Kompatibilität des Fluids mit dem Material der Membran.

Dieses Kapitel behandelt den Aufbau verschiedener flexibler Fluidaktoren [165, 166], die Modellierung der Momenten- und Volumenkennfelder sowie das Federverhalten im pneumatischen und hydraulischen Betrieb. Das Verständnis des Aktorverhaltens und die hergeleiteten Modelle bildet später die Grundlage für Sensorauswahl, Momentenschätzung und Gelenkregelung.

\subsection{Aufbau flexibler Fluidaktoren}

Flexible Fluidaktoren wirken entweder nach dem Kontraktions- oder Expansionsprinzip. Beim Kontraktionsprinzip zieht sich der Aktor entlang seiner Wirkrichtung zusammen und dehnt sich dabei quer dazu aus, ein Aktor nach dem Expansionsprinzip verhält sich umgekehrt dazu. Die Geometrie und die Materialien der elastischen Kammer sind für den jeweiligen Anwendungsfall optimiert.

\subsubsection{Kontraktionsprinzip}

Auf das Kontraktionsprinzip optimierte Aktoren (Abbildung 3.1) bieten eine hohe Leistungsdichte und besitzen meist eine längliche Ausgangsform. Sie verkürzen sich bei maximaler Druckbeaufschlagung um $25-40 \%$. Dieses Verhalten wird durch den Aufbau der Aktoren unterstützt.

Ein typischer Aufbau besteht aus einer von einem diagonal verwobenen Netzschlauch umspannten länglichen Gummiblase wie z.B. im McKibben Actua- 


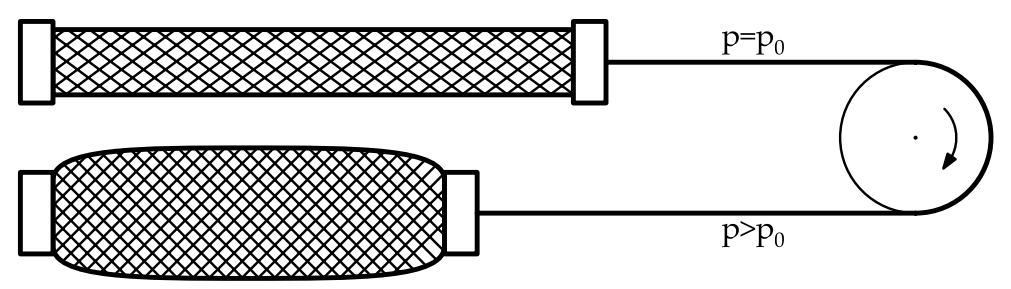

Abbildung 3.1: Antagonistische Anordnung zweier flexibler Fluidaktoren nach dem Kontraktionsprinzip (Aktordruck $p$, Umgebungsdruck $p_{0}$ ) nach [143, 178].

tor [99, 100, 101]. Dieser wurde auch von der Fa. Bridgestone als Rubbertuator [161] mit einer optimierten Netzstruktur [160] hergestellt. Varianten dieser Aktoren sind auch doppelt wirkend [138] ausgeführt oder mit Sensoren zur Zugkraftmessung [159] ausgestattet. Von den McKibben-Aktoren abgeleitete pneumatic muscle actuators werden in den Unterarmen der Salford Hand [26], ähnliche air muscles in der Shadow Hand [193] eingesetzt.

Aktoren mit einem einteiligen Aufbau der Außenhülle bieten den Vorteil geringerer Reibungsverluste. Die Fa. Festo integriert in ihrem fluidic muscle MAS [50] die Außenhaut des Aktors $\mathrm{zu}$ einem faserverstärkten elastischen Schlauch. Ganz auf Materialausdehnung verzichten die pleated pneumatic artificial muscles (PPAM) [38], deren Hülle aus einem Aramidgewebe mit einer Abdichtung aus Polypropylen besteht. Die Außenhaut ist in Längsrichtung gefaltet, so kann sich der Aktor in Querrichtung ohne Materialdehnung ausbreiten.

Da kontrahierende Aktoren keine Quer- oder Druckbelastungen aufnehmen können, erfolgt ihr Einsatz meist in einer antagonistischen Anordnung, bei der an jedem Gelenk zwei Aktoren in entgegengesetzter Zugrichtung wirken (Abbildung 3.1). Ihr Verhalten wurde in mehreren Armen [143, 178, 184] und Beinen [190, 192] untersucht. Vorteilhaft ist hierbei, dass sich über die Differenz der Aktordrücke die Position und über die Summe der Aktordrücke die Steifigkeit des Gelenks gleichzeitig einstellen lassen.

\subsubsection{Expansionsprinzip}

Beim Expansionsprinzip dehnt sich der Aktor durch Erhöhung des Fluiddrucks entlang seiner Hauptachse aus. Solche Aktoren werden z.B. als flache Hebekissen oder in Balgenform zur Luftfederung im Nutzfahrzeugen eingesetzt. Kleine Ausführungen solcher Aktoren mit ca. $10 \mathrm{~mm}$ Durchmesser können auch zum Beugen der Fingergelenke einer künstlichen Hand (Abbildung 3.2) verwendet werden [167, 168, 169, 170, 171, 173].

Eine weitere Variante der Aktoren nach dem Expansionsprinzip sind flexible microactuators (FMA), deren Schlauchform entlang der Längsachse sternförmig in drei Kammern unterteilt ist. Durch unterschiedliche Druckbeaufschlagung dieser Kammern beugt sich der Aktor in unterschiedliche Richtungen [39, 48, 70, 71, 107, 

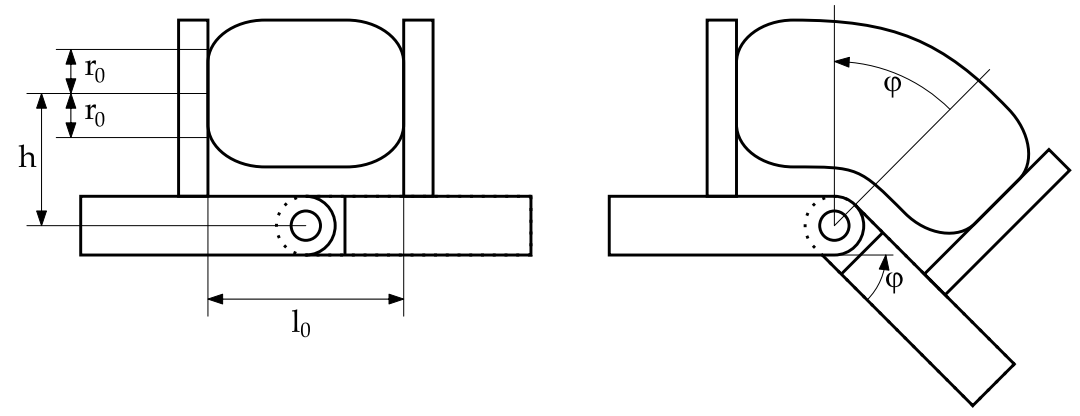

Abbildung 3.2: Schematische Darstellung eines flexiblen Fluidaktors nach dem Expansionssprinzip in einem Fingergelenk.

182, 183]. In geeigneter Anordnung können diese Aktoren auch als elastische Finger einer Hand genutzt werden.

\subsection{Kenngrößen und Modellierung des Gelenkaktors}

Die für die Beschreibung des Aktors wichtigsten Größen sind das Gelenkmoment und das Aktorvolumen. In diesem Abschnitt werden für den flexiblen Gelenkaktor aus Abbildung 3.2 Modelle für die Abhängigkeit von Aktordruck und Gelenkwinkel diskutiert und an Hand von Messdaten validiert.

\subsubsection{Momentenkennfeld}

Das Gelenkmoment ist die zentrale Größe sowohl für die Winkelbeschleunigung $\ddot{\varphi}$ eines sich frei bewegenden Gelenks als auch für die Bestimmung der Kräfte, die auf ein mit dem Finger in Kontakt stehendes Objekt ausgeübt werden.

\section{Qualitatives theoretisches Modell}

Das von einem flexiblen Fluidaktor auf das in Abbildung 3.2 gezeigte Gelenk ausgeübte Drehmoment $M_{\mathrm{A}}$ lässt sich aus Rückstellmoment, Druck $p$ des Aktors, Gelenkgeometrie und Gelenkwinkel $\varphi$ abschätzen. Ein Modell hierfür wird in [12,119] ausführlicher hergeleitet. Mit dem durch den Parameter $a_{00}$ beschriebenen Moment bei gestrecktem Gelenk und einer linearen Drehfeder mit der Federkonstante $a_{01}$ wird das Rückstellverhalten des drucklosen Aktors in $M_{0}$ beschrieben. $M_{1}$ ist das durch den Aktordruck auf die kreisförmigen Endflächen über die Aktorhalter ausgeübte Moment. Das durch Innendruck und Gelenkwinkel veränderte elastische Verhalten wird in $M_{2}$ mit der druckabhängigen Federkonstante $a_{11}$ berücksichtigt. 


$$
\begin{aligned}
M_{0}(\varphi) & =a_{00}+a_{01} \cdot \varphi \\
M_{1}(p) & =F \cdot h=p \cdot A_{0} \cdot h=p \cdot \pi \cdot r_{0}^{2} \cdot h=a_{10} \cdot p \\
M_{2}(\varphi, p) & =a_{11} \cdot \varphi \cdot p \\
M_{\mathrm{A}}(\varphi, p) & =M_{0}(\varphi)+M_{1}(p)+M_{2}(\varphi, p) \\
& =a_{00}+a_{01} \cdot \varphi+a_{10} \cdot p+a_{11} \cdot \varphi \cdot p
\end{aligned}
$$

Aus der Summation der Einzelmomente ergibt sich das Aktormoment $M_{\mathrm{A}}$. Nach Zusammenfassung der Einzelterme kann es mit Hilfe der vier Parameter $a_{00} \ldots a_{11}$ aus dem Druck $p$ und dem Gelenkwinkel $\varphi$ angenähert werden.

\section{Approximationsmodell aus Messdaten}

Mit einem Teststand [11] wurde für einen Aktor das Drehmomentenkennfeld $M_{\mathrm{A}}$ (Abbildung 3.3) eines Aktors in Abhängigkeit vom Gelenkwinkel $\varphi$ und dem Aktordruck $p$ gemessen. Hierbei wird ein Gelenk mit Aktor durch einen Schrittmotor positioniert, der Aktordruck wird durch ein Druckregelventil konstant gehalten und das durch das Gelenk ausgeübte Moment mit einer Messdose aufgenommen. Die Messung erfolgt über den gesamten Bewegungsbereich des Gelenks und den Arbeitsdruckbereich des Aktors [13]. Abschließend wurden die Parameter $a_{00} \ldots a_{11}$ der Gleichung (3.4) nach der Methode der kleinsten Fehlerquadrate [30, 197] ermittelt. Der maximale Fehlerbetrag $\left|\Delta M_{\mathrm{A}}\right|$ des Modells ist bei den dargestellten Messdaten 0,1 Nm , dies entspricht bei einem Maximalmoment von $0,6 \mathrm{Nm}$ einem relativen Fehler von $\frac{0,1 \mathrm{Nm}}{0,6 \mathrm{Nm}}=16,5 \%$. Im Mittel liegt der Fehlerbetrag bei $0,02 \mathrm{Nm}(2,8 \%)$.

\subsubsection{Volumenkennfeld}

Die Kenntnis des Aktorvolumens ist insbesondere im Anwendungsfall der Hydraulik wichtig, wo das Bewegungsverhalten durch den Volumenstrom des inkompressiblen Mediums bestimmt ist. Im pneumatischen Aktorbetrieb kann ein geschätztes Aktorvolumen zur Adaption eines Druckreglers vorteilhaft eingesetzt werden [62].

\section{Qualitatives theoretisches Modell}

Ebenso wie das Drehmoment hängt das Volumen des Aktors in Abbildung 3.2 primär vom Aktordruck und der Winkelstellung des Gelenks ab. Bisherige Modelle [116] berücksichtigen jedoch nur den winkelabhängigen Anteil. Das hier vorgestellte Modell verbessert diesen Ansatz, indem er durch den druckabhängigen Beitrag erweitert wird.

In der Ruhelage wird der Aktor als ein Zylinder mit Radius $r_{0}$ und Länge $l_{0}$ angenähert. Die Beugung des Gelenks wird durch einen Torussektor mit dem Gelenkwinkel $\varphi$ und dem Abstand $h$ zur Rotationsachse beschrieben, wobei die Quer- 


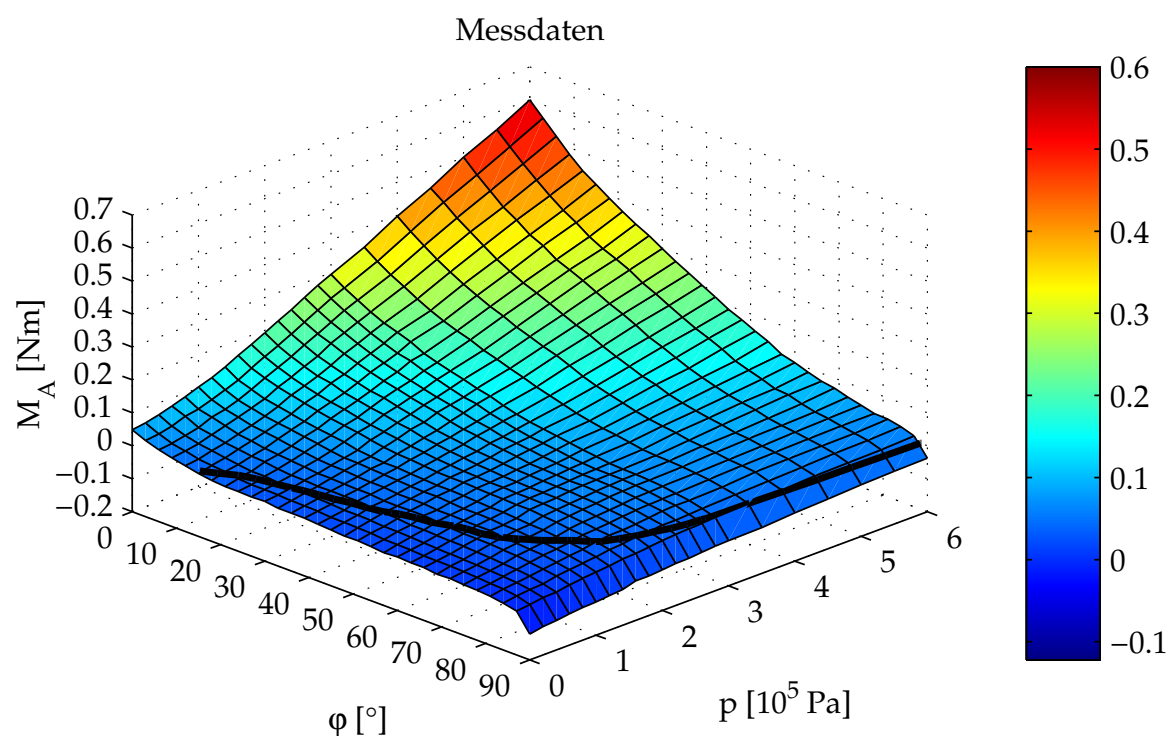

Abbildung 3.3: Messdaten: Drehmoment $M_{\mathrm{A}}$ des Aktors in Abhängigkeit von Gelenkwinkel $\varphi$ und Aktordruck $p$ und hervorgehobene Nullmomentenkennlinie.

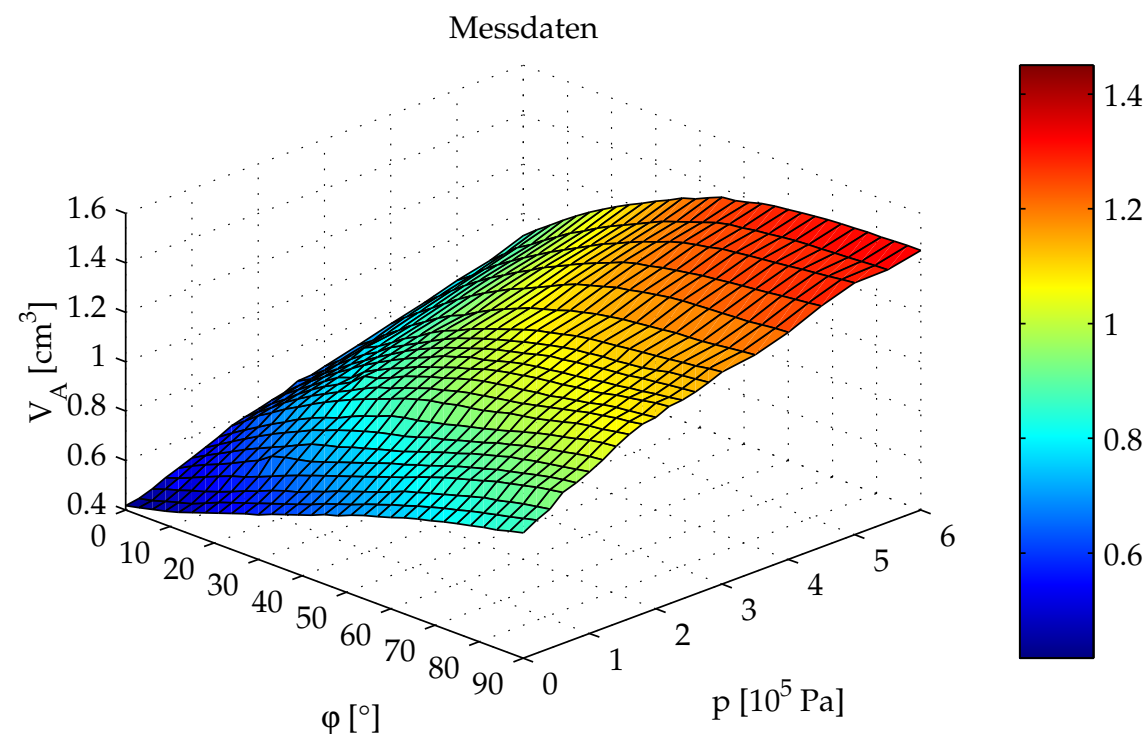

Abbildung 3.4: Messdaten: Volumen $V_{\mathrm{A}}$ des Aktors in Abhängigkeit von Gelenkwinkel $\varphi$ und Aktordruck $p$. 
schnittsflächen $A$ von Zylinder und Torussektor als gleich groß angenommen werden. Die linear mit dem Druck zunehmende Querschnittsfläche wird über die relative Flächenausdehnung $E$ ermittelt.

$$
\begin{aligned}
A & =A_{0} \cdot(1+E \cdot p)=\pi \cdot r_{0}^{2} \cdot(1+E \cdot p) \\
V_{Z} & =A \cdot l_{0} \\
V_{T} & =A \cdot h \cdot \varphi \\
V_{\mathrm{A}} & =V_{Z}+V_{T}=A \cdot\left(l_{0}+h \cdot \varphi\right) \\
& =\pi \cdot r_{0}^{2} \cdot(1+E \cdot p) \cdot\left(l_{0}+h \cdot \varphi\right) \\
& =b_{0}+b_{1} \cdot \varphi+b_{2} \cdot p+b_{3} \cdot \varphi \cdot p
\end{aligned}
$$

Durch Umstellung der Gleichungen kann das Volumenverhalten ähnlich dem Momentenverhalten in eine Darstellungsform mit den vier Parametern $b_{0} \ldots b_{3}$ gebracht werden.

\section{Approximationsmodell aus Messdaten}

Für die Bestimmung des Aktorvolumens wurde der Messaufbau aus dem vorangegangenen Abschnitt angepasst: Der Aktor wird durch ein transparentes Steigrohr mit einem inkompressiblen Medium (Silikonöl) befüllt. Die Druckerzeugung und -regelung bleibt pneumatisch. Aus dem Flüssigkeitsstand im Steigrohr wird in gleicher Vorgehensweise wie bei der Momentenmessung das winkel- und druckabhängige Volumenkennfeld (Abbildung 3.4) ermittelt [119].

Der maximale Approximationsfehler für das Modell aus Gleichung (3.8) beträgt für die gezeigten Messdaten $0,16 \mathrm{~cm}^{3}(11 \%)$, im Mittel liegt er bei $0,05 \mathrm{~cm}^{3}(3 \%)$.

\subsection{Passive Momententrajektorien}

Die meisten in künstlichen Händen eingesetzten Aktoren zeigen ein sehr steifes Verhalten. Finger weichen bei Ausübung einer Kraft nicht oder nur minimal aus. Ist ein nachgiebiges Verhalten gewünscht, so ist dies entweder mit Hilfe von elastischen Elementen im Antriebsstrang oder durch eine Regelung zu realisieren. Im Gegensatz dazu ist die Nachgiebigkeit eine inhärente Eigenschaft flexibler Fluidaktoren, die zudem vom eingesetzten Medium abhängt.

In den folgenden Abschnitten wird erstmalig das Verhalten eines solchen Aktors bei Befüllung mit einer konstanten Fluidmasse und einem von außen wirkenden Moment analysiert. Hierfür wird zuerst im Volumenkennfeld die Beziehung zwischen Winkel und Druck bei unterschiedlichen Fluidmassen betrachtet. Diese Trajektorien werden anschließend auf das Momentenkennfeld des Aktors übertragen. Die Analyse wird am Beispiel eines idealen Gases und einer inkompressiblen Flüssigkeit durchgeführt. 


\subsubsection{Pneumatischer Betrieb}

Für den pneumatischen Betrieb wird das Verhalten eines idealen Gases mit der allgemeinen Gasgleichung (3.9) beschrieben.

$$
p \cdot V_{\mathrm{A}}=m \cdot R \cdot T_{\mathrm{R}}
$$

Dabei ist $R$ die allgemeine Gaskonstante $\left(287,2 \frac{\mathrm{J}}{\mathrm{kg} \cdot \mathrm{K}}\right)$ und $T_{\mathrm{R}}$ die Raumtemperatur $(296 \mathrm{~K})$. Es wird eine isotherme Zustandsänderung nach dem Gesetz von BoyleMariotte in Gleichung (3.10) angenommen, wonach das Produkt aus Druck und Volumen einer bestimmten Masse $m$ eines idealen Gases bei konstanter Temperatur $T$ unverändert bleibt.

$$
p \cdot V_{\mathrm{A}}=\text { konstant }_{T=\text { konstant }, m=\text { konstant }}
$$

So kann aus dem im Abschnitt 3.2 ermittelten Volumenkennfeld ein Winkelkennfeld $\varphi(p, m)$ für die sich bei unterschiedlichen $p$ und $m$ ergebenden Gelenkwinkel berechnet werden (Abbildung 3.5). Die schwarz hervorgehobenen Linien zeigen dabei Trajektorien gleicher Fluidmasse. Für dieses Verhalten wird vorausgesetzt, dass das Totvolumen im Schlauch zwischen Ventil und Aktor deutlich kleiner als das Aktorvolumen ist. Mit steigendem Totvolumen wird die Druckänderung immer geringer.

Diese Kennlinien mit konstanter Fluidmasse lassen sich wiederum auf das Momentenkennfeld des Aktors übertragen (Abbildung 3.6). Deutlich sichtbar ist, dass sich mit der Verringerung des Gelenkwinkels ein Druckanstieg ergibt. Bis zu einem Ausgangsdruck von fast $4 \cdot 10^{5} P a$ enden alle Kennlinien bei $\varphi=0^{\circ}$. Das Gelenk kann also ohne Erreichen des Maximaldrucks von $6 \cdot 10^{5} \mathrm{~Pa}$ vollständig geöffnet werden.

\subsubsection{Hydraulischer Betrieb}

Im hydraulischen Fall wird ein nicht kompressibles Fluid vorausgesetzt, dessen Volumen unabhängig vom Druck konstant bleibt. Die Trajektorien konstanter Fluidmasse können damit in Trajektorien konstanten Volumens übertragen werden. Die Daten entsprechen damit dem in die Form $\varphi\left(p, V_{\mathrm{A}}\right)$ umgestellten Volumenkennfeld (Abbildung 3.7). Im Kennfeld sind die Bahnen gleichen Volumens schwarz markiert.

Die Übertragung dieser Kennlinien auf das Momentenkennfeld zeigt in Abbildung 3.8 ein gegenüber der Pneumatik steiferes Verhalten, da nur noch die Nachgiebigkeit des Aktors zu berücksichtigen ist. Wird das Gelenk durch ein von außen wirkendes Moment geöffnet, so steigt der Druck im Aktor deutlich schneller als im pneumatischen Betrieb. Die meisten Kennlinien enden bei $p=6 \cdot 10^{5} \mathrm{~Pa}$, ohne dass das Gelenk vollständig geöffnet wird. Es besteht also viel eher als bei der Pneumatik die Gefahr, dass durch externe Drehmomente ein maximaler Aktordruck überschritten wird. 


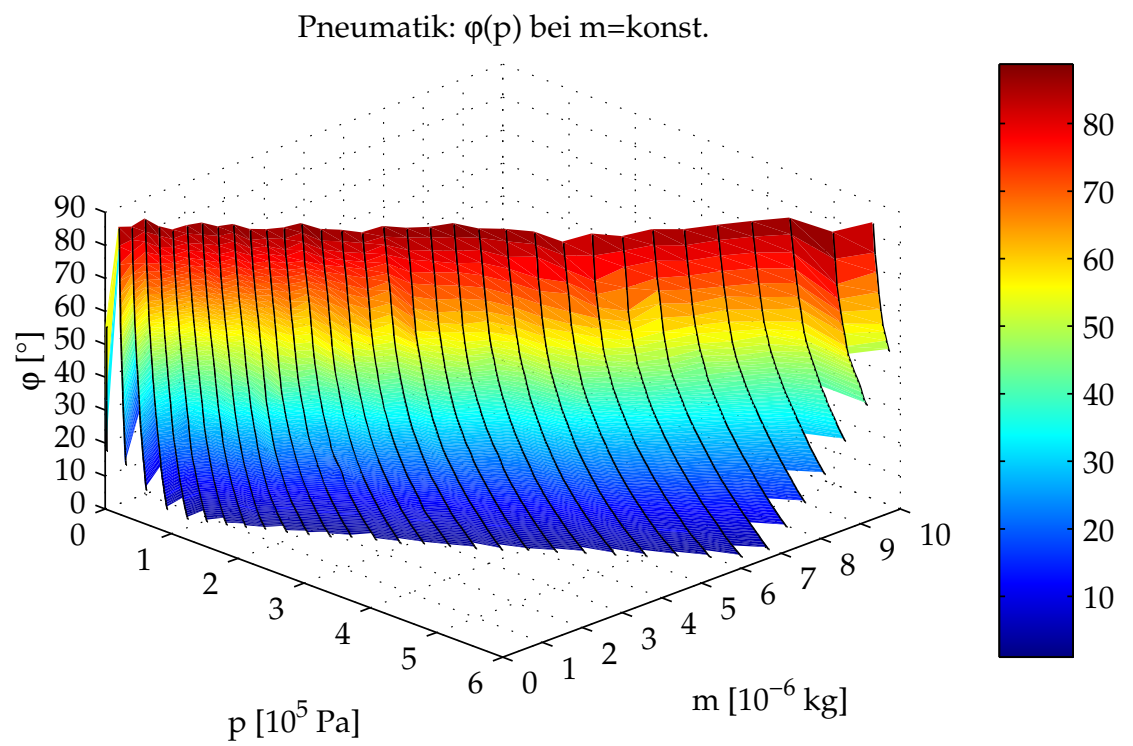

Abbildung 3.5: Aus Messdaten berechnetes Winkelkennfeld $\varphi(p, m)$ eines mit einem idealen Gas betriebenen flexiblen Fluidaktors mit $m=$ konstant. Die Linien gleicher Fluidmasse sind schwarz eingezeichnet.

Pneumatik: Momententrajektorien bei $\mathrm{p}^{*} \mathrm{~V}_{\mathrm{A}}=$ konst.

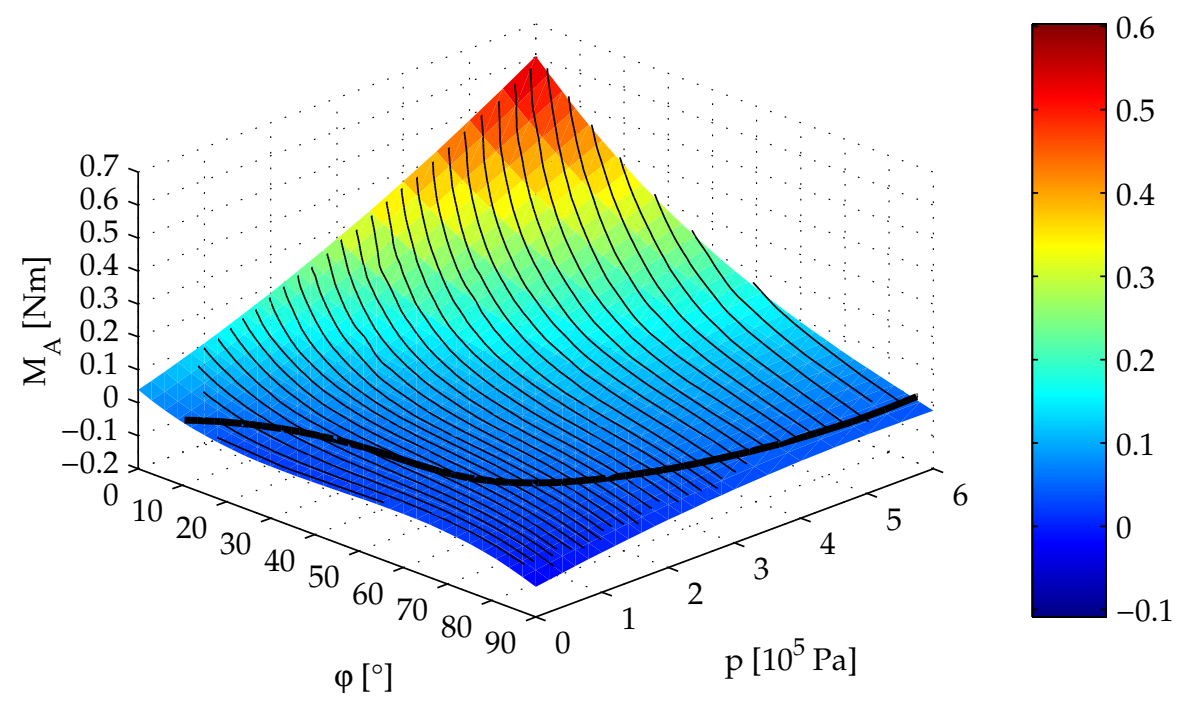

Abbildung 3.6: Aus Messdaten berechnete Momententrajektorien $\left.M_{\mathrm{A}}(\varphi, p)\right|_{T=k o n s t a n t, m=k o n s t a n t}$ eines mit einem idealen Gas betriebenen flexiblen Fluidaktors $\left(p \cdot V_{\mathrm{A}}=k o n s t a n t\right)$. Die Nullmomentenkennlinie ist dick hervorgehoben. 


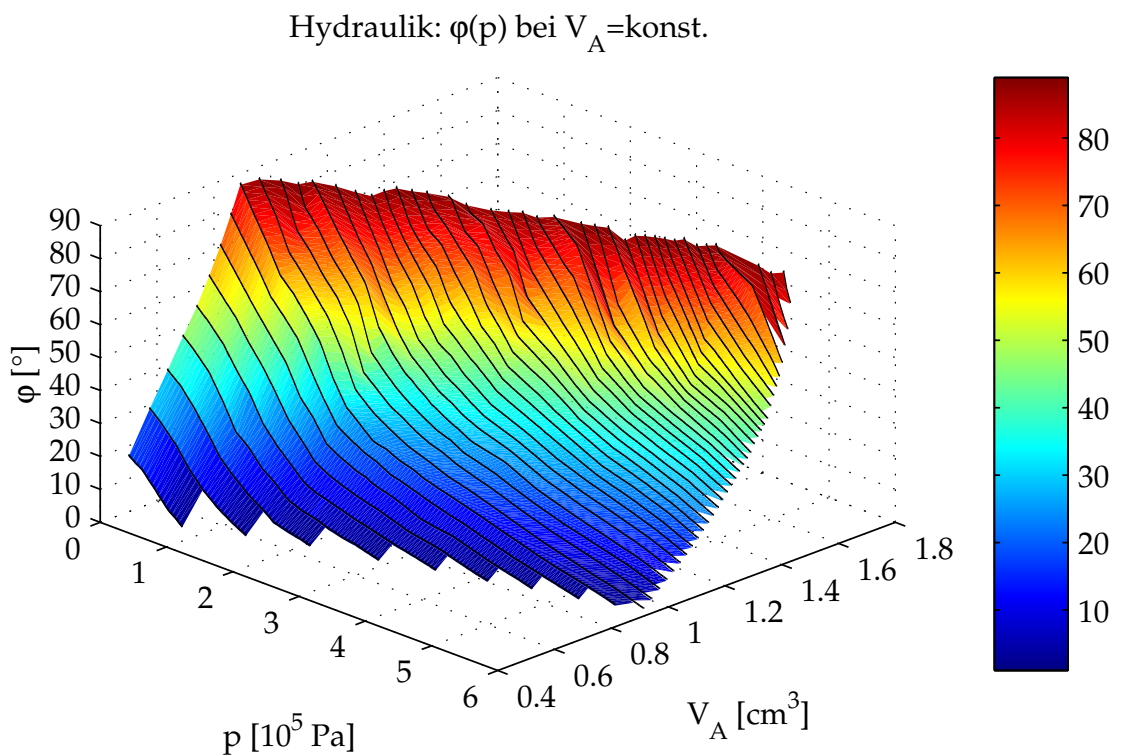

Abbildung 3.7: Messdaten des Winkelkennfelds $\varphi\left(V_{\mathrm{A}}, p\right)$ eines mit einem inkompressiblen Medium betriebenen flexiblen Fluidaktors mit $V_{\mathrm{A}}=$ konstant. Die Linien gleichen Fluidvolumens sind schwarz eingezeichnet.

Hydraulik: Momententrajektorien bei $\mathrm{V}_{\mathrm{A}}=$ konst.

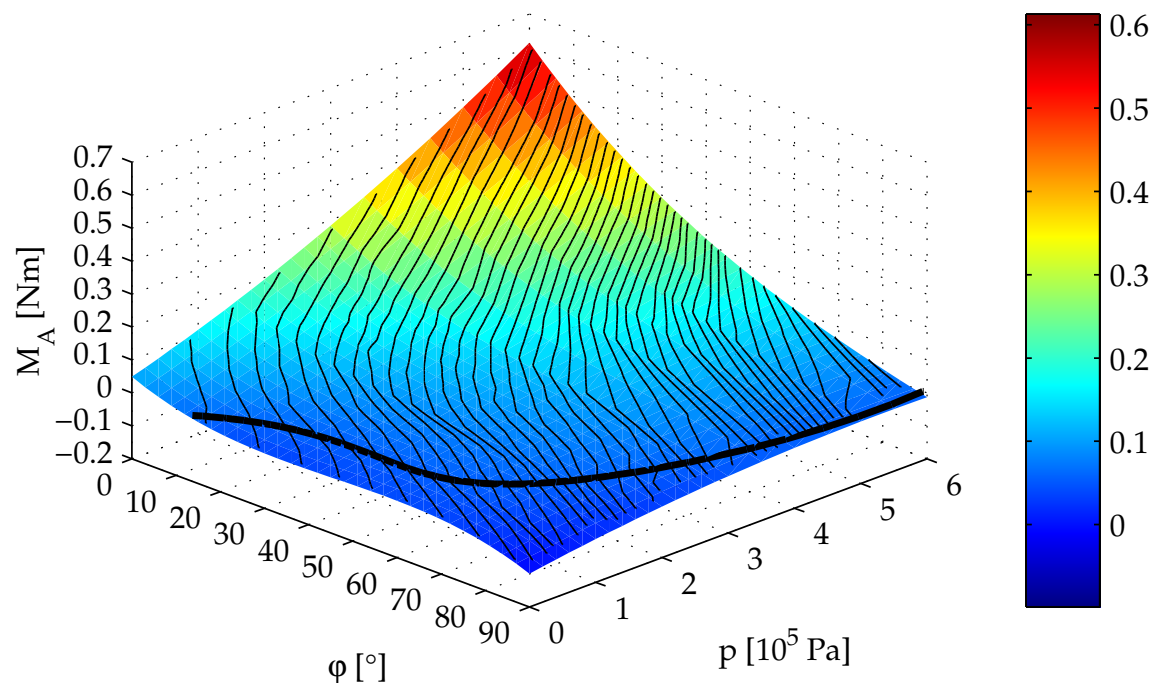

Abbildung 3.8: Aus Messdaten berechnete Momententrajektorien $M_{\mathrm{A}}(\varphi, p)$ eines mit einem inkompressiblen Medium betriebenen flexiblen Fluidaktors mit $V_{\mathrm{A}}=$ konstant. Die Nullmomentenkennlinie ist dick hervorgehoben. 


\subsection{Medienabhängiges Steifigkeitsverhalten}

Die Beschreibung der Momententrajektorien wird in diesem Abschnitt um das passive Steifigkeitsverhalten eines Aktors in Analogie zu einer nichtlinearen Drehfeder erweitert. Die Steifigkeit $c(\varphi, m)$ dieser Feder wird aus der partiellen Ableitung der Momententrajektorien nach Gleichung (3.11) berechnet, die zu $c$ reziproke Größe $k$ wird als Nachgiebigkeit bezeichnet.

$$
c(\varphi, m)=\frac{1}{k(\varphi, m)}=-\left.\frac{\delta M_{\mathrm{A}}(\varphi, m)}{\delta \varphi}\right|_{T=k o n s t a n t, m=k o n s t a n t}
$$

Aus Gründen der Anschaulichkeit wird auch $c(\varphi, m)$ nach entsprechender Rückrechnung als $c(\varphi, p)$ dargestellt.

\subsubsection{Pneumatischer Betrieb}

Das pneumatische Federkonstantenkennfeld (Abbildung 3.9) zeigt im Umfeld der statischen Kennlinie ein sehr flach ansteigendes Verhalten. Das Gelenk weist damit in diesem Bereich nur eine geringe Progression der Federsteifigkeit auf, es verhält sich also annähernd wie eine lineare Feder. Daraus ist zu schließen, dass ein Finger mit solchen Aktoren beim Berühren eines Objekts zunächst sehr leicht nachgibt und keine großen Kräfte auf das Objekt ausübt.

Dagegen ist beim stark gestreckten Gelenk ein deutliches progressives Verhalten zu erkennen, das mit dem Aktordruck ansteigt. Wird der Aktor beim Greifen in diesem Bereich betrieben, so steigt das Gelenkmoment auch mit kleinen Positionsänderungen schnell an.

\subsubsection{Hydraulischer Betrieb}

Im Fall der Hydraulik zeigt das Kennfeld (Abbildung 3.10) ebenfalls ein weiches Verhalten im direkten Umfeld der Nullmomentenkennlinie. Der wesentliche Unterschied zum pneumatischen Betrieb ist die fast doppelt so große maximale Federsteifigkeit.

Die Trajektorien weichen in gleicher Weise wie die pneumatischen und hydraulischen Momentenkennlinien voneinander ab. Ein hoher Anteil von ihnen erreicht den Maximaldruck des Aktors bereits, ohne dass das Gelenk vollständig geöffnet ist. Dabei ist gerade bei im unbelasteten Zustand stark gebeugten Gelenken nur eine geringe Progression zu beobachten, bevor der Maximaldruck erreicht wird. Deutlicher ausgeprägt ist das Progressionsverhalten im mittleren Ruhelagebereich. 


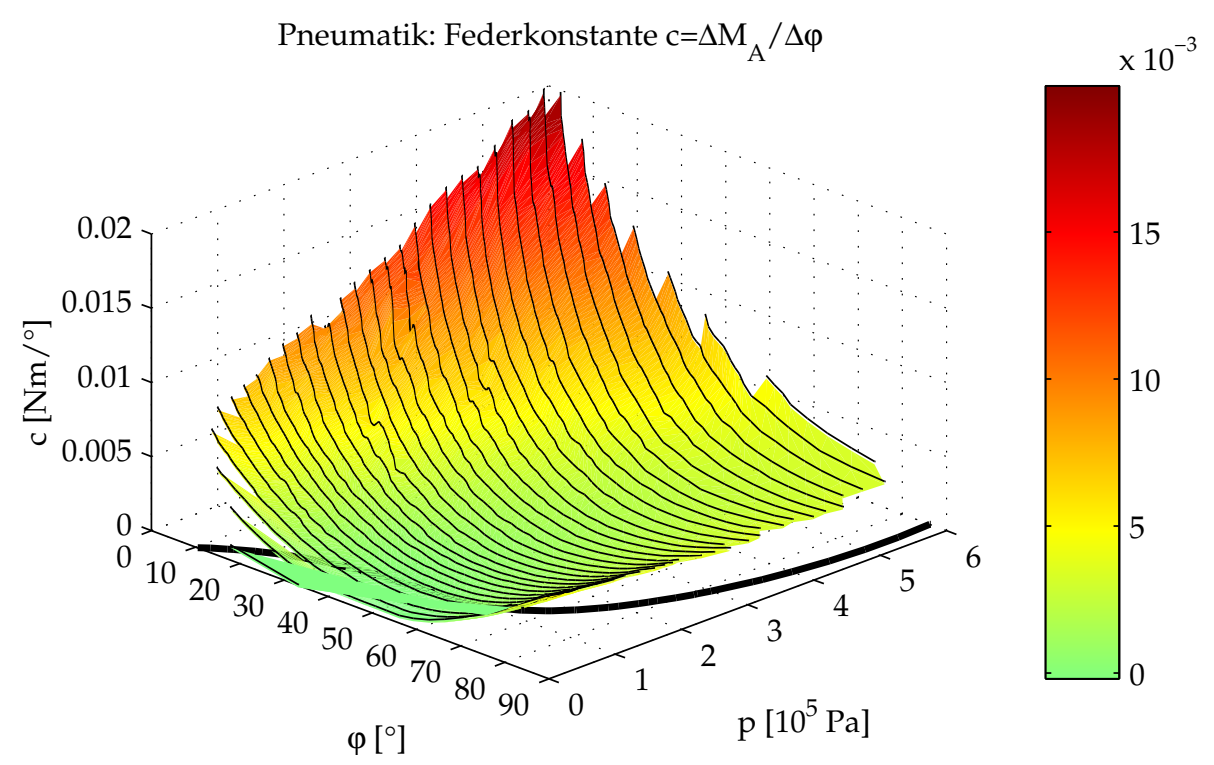

Abbildung 3.9: Federkonstantenkennfeld Pneumatik mit $m=$ konstant .
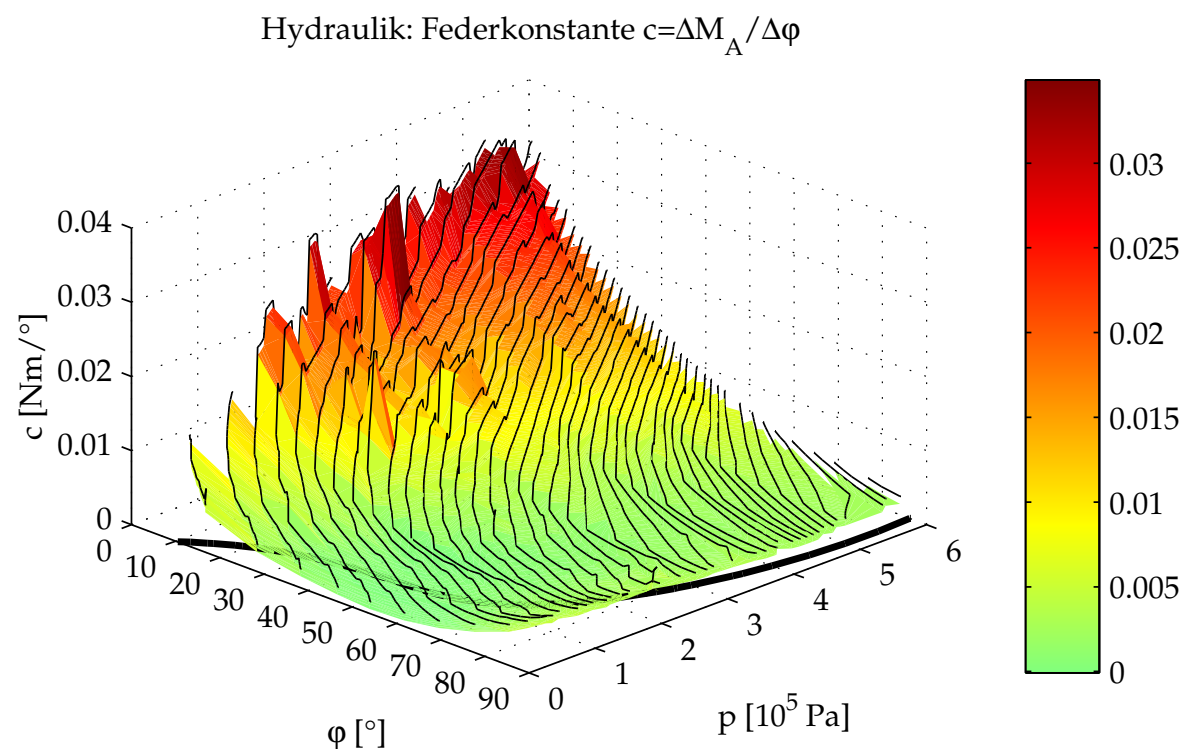

Abbildung 3.10: Federkonstantenkennfeld Hydraulik mit $V_{\mathrm{A}}=$ konstant . 


\section{Auswahl und Optimierung der Sensoren}

Erst die Sinnesorgane erlauben dem Menschen die feinfühlige Wahrnehmung seiner Umgebung und die präzise koordinierte Bewegung seiner Hände. Dies gilt gleichermaßen für die Sensoren einer künstlichen Hand, ohne die die Handsteuerung keine Rückmeldung über den Zustand der Hand und ihre Interaktion mit der Außenwelt erhält. Die Ausstattung mit geeigneten Sensoren ist die Grundlage für die Regelung der einzelnen Gelenke und die Koordination von Bewegungen und Greifvorgängen der Hand [123, 124].

In diesem Kapitel werden Winkelsensoren, taktile Sensoren sowie Gelenkmomentensensoren für den Einsatz in von flexiblen Fluidaktoren angetriebenen künstlichen Händen vorgestellt. Für die drei gewählten Sensorklassen erfolgt nach einer Übersicht verschiedener Messprinzipien und einer Zusammenstellung der jeweils spezifischen Sensoranforderungen die Auswahl eines geeigneten Sensorprinzips und die Auslegung für den gegebenen Anwendungsfall.

\subsection{Winkelsensoren}

Die Bestimmung der Gelenkpositionen einer künstlichen Hand durch Winkelsensoren ist insbesondere in der Robotik von hoher Bedeutung, da eine visuelle Erfassung der Handhaltung mit Kamera und Bilderkennungsverfahren sehr aufwändig und nur unter genau definierten Umgebungen zuverlässig realisierbar ist [141, 142, 187].

Die ermittelten Winkelpositionen sind Grundlage für die Positionsregelung der Gelenke und die Berechnung der kartesischen Fingerpositionen aus den kinematischen Daten der Hand zur vorausschauenden Kollisionsvermeidung mit der Umgebung.

In diesem Abschnitt werden mehrere Messverfahren für Gelenkpositionen vorgestellt, die Anforderungen an Winkelsensoren für Gelenke mit flexiblen Fluidaktoren zusammengetragen und nach einer Auswahl des Sensorprinzips die Realisierung und Optimierung der Sensoren diskutiert.

Die Verfahren zur Winkelpositionsmessung der Fingergelenke sind in zwei Hauptgruppen zu unterteilen: gelenkexterne Sensoren messen die Position indirekt an der Antriebsseite, während gelenkinterne Sensoren eine abtriebsseitige Messung im direkten Umfeld des Gelenks erlauben. Erstere werden daher meist bei externen Aktoren mit einer Getriebe- oder Spindeluntersetzung verwendet. In 
diesem Fall muss auch auf die beengten Platzverhältnisse innerhalb der Finger keine Rücksicht mehr genommen werden.

\subsubsection{Gelenkinterne Sensoren}

Der Hauptvorteil gelenkinterner Sensoren ist wegen der direkten Verbindung die geringe Verfälschung der Messergebnisse durch das Übertragungsverhalten eines Antriebsstrangs. Herausforderungen sind jedoch die beengten Platzverhältnisse in den Gelenken und Fingergliedern, die mechanische Integration mit einer möglichst geringen Schwächung der Gelenkstruktur sowie die erforderliche Verkabelung bewegter Komponenten.

\section{Potentiometer}

Ein einfach zu realisierendes Messverfahren für Gelenkwinkel ist die Messung mit einem als Spannungsteiler geschalteten linearen Drehpotentiometer, dessen Ausgangssignal am Schleifkontakt proportional zum Gelenkwinkel ist. Der geometrische Aufbau verfügbarer Potentiometer verlangt eine in der Fingermitte liegende Gelenkachse, bei zu weit palmar oder dorsal liegenden Achsen steht ein Teil des Potentiometers über die Fingerkontur hinaus. Ein weiterer Nachteil ist die Erhöhung der Reibung durch den Schleifkontakt. Die Potentiometer in der DLRHand II [22] sind Sonderanfertigungen aus leitfähigem Kunststoff. Durch sie kann bei der Initialisierung auf eine Referenzfahrt für die Motorencoder und auf die Modellierung der Winkelfehler durch Elastizitäten im Antrieb verzichtet werden.

\section{Resistive Beugesensoren}

Resistive Beugesensoren werden typischerweise als auf eine flexible PolyimidTrägerfolie aufgedruckte Widerstands- und Leiterstruktur realisiert [61]. Beim Biegen des Sensors wird die Widerstandsschicht elastisch gedehnt und ihr Widerstand steigt. Solche Sensoren werden z.B. in den Cyberglove Datenhandschuhen [79] entlang des Fingerrückens eingesetzt. In ähnlicher Weise werden sie in der UB-Hand III der Universität Bologna [18, 117] verwendet. Die dort realisierte Fingerstruktur hat keine definierten Gelenkachsen [118], womit viele andere Messprinzipien nicht einsetzbar sind.

Beim Langzeitbetrieb resistiver Beugesensoren zeigt sich insbesondere bei kleinen Beugeradien eine Abnutzung, die eine regelmäßige Rekalibrierung erfordert [116]. Für Datenhandschuhe ist dies unkritisch, da dieser ohnehin nach jedem Anziehen auf die Geometrie und Beweglichkeit der Hand des Trägers zu kalibrieren ist.

\section{Optische Beugesensoren}

Beugesensoren können ebenso aus flexiblen Lichtleitern aufgebaut werden, deren Lichtleitfähigkeit mit engeren Biegeradien abnimmt [202]. Verwendet wurde dieses Prinzip in den Datenhandschuhen der Fa. VPL Research [203]. 


\section{Position Sensitive Detector}

In der ersten Generation der DLR-Hand [23, 113] wird ein linearer PSD (position sensitive detector, position sensing device) und eine Scheibe mit einer spiralförmigen Schlitzblende zur Winkelmessung im Gelenk eingesetzt. Durch die Rotation der Scheibe ändert sich die Position des von einer Infrarot-LED beleuchteten Bereichs auf der Oberfläche des PSD. Aus dem positionsabhängigen Ausgangssignal wird die Gelenkposition bestimmt.

\section{Magnetfeldsensoren}

In Verbindung mit Permanentmagneten werden auch Magnetfeldsensoren wie z.B. Hallsensoren als Sensorprinzip zur direkten Winkelmessung herangezogen. Magnet und Sensor werden an beiden Gelenkseiten so zueinander angeordnet, dass sich die gemessene magnetische Flussdichte über den geforderten Messbereich möglichst linear ändert. Dieses Messverfahren ermöglicht nach einer Kalibrierung eine absolute Positionsmessung.

In der Utah/MIT-Hand [81, 199] werden das Gelenk ausfüllende Scheibenmagnete und seitlich dazu angeordnete Hallsensoren zur Winkelmessung verwendet. Die in [28] für die RTR-Hand vorgeschlagene Anordnung nutzt eine Reihe von am Antriebsgestänge angebrachten Magneten, die durch die Bewegung des Antriebsgestänges linear an einem fest stehenden Hallsensor vorbei bewegt werden.

\subsubsection{Externe Sensoren}

Erfolgt der Antrieb eines Gelenks durch einen außerhalb des Fingers liegenden Aktor, so bieten sich auch antriebsseitige Sensoren an. Sie profitieren von den meist vorhandenen Untersetzungen im Antrieb und bieten eine hohe Auflösung. Ihre Genauigkeit ist jedoch durch das meist nur teilweise bekannte last-, positions-, spiel- und zeitabhängige Übertragungsverhalten des Antriebsstrangs begrenzt. Die Korrektur solcher Messfehler erfordert also ein Modell des Übertragungsverhaltens sowie die Messung der einfließenden Größen. Externe Winkelsensoren werden daher in manchen Fällen durch zusätzliche gelenkinterne Winkelsensoren ergänzt [22]. Ein weiterer Vorteil liegt in den weniger beengten Platzverhältnissen außerhalb der Finger und einer vereinfachten Verkabelung der Sensoren, da die Leitungsführung durch die Gelenke entfällt.

\section{Optische und magnetische Inkrementalgeber}

Als Positionsmessverfahren für motorgetriebene Fingergelenke bieten sich Inkrementalgeber an, die direkt am Motor mit hoher Drehzahl und geringer Winkelauflösung messen. Sie liefern Impulsfolgen zweier phasenversetzter Aufnehmer, aus denen die Anzahl der Drehschritte und die Drehrichtung bestimmt werden. Dieses inkrementelle Messverfahren erfordert zur Ermittlung der Gelenkposition eine Kalibrierung durch eine Referenzfahrt gegen einen Endanschlag oder einen Kontakt mit bekannter Winkelposition. Ebenso muss die Schlupffreiheit der mechanischen Verbindung zum Fingergelenk sichergestellt sein. 
Optische Ausführungen dieses Winkelmessverfahrens sind z.B. in der Karlsruher Hand II in Verbindung mit Spindeltrieben [55, 56] anzutreffen. Die Encoder in der Gifu-Hand [96] sind mit Permanentmagneten und Hallsensoren aufgebaut.

\section{Linearpotentiometer}

Die Druckerzeugung für die hydraulisch bewegte TUM-Hand [128] erfolgt mittels externer Zylinder und elektromotorisch betätigter Spindeltriebe. Die Winkelmessung erfolgt durch Linearpotentiometer an den Spindeltrieben. Die Genauigkeit dieses Verfahrens leidet an der Komplexität der Übertragungsstrecke. Faktoren wie das viskoelastische Verhalten des Hydraulikschlauchs können unter Zuhilfenahme einer Druckmessung ausgeglichen werden. Mit dem durch Undichtigkeiten in den Zylinderdichtungen steigenden Luftanteil steigt auch die Kompressibilität des Hydraulikmediums. Dies kann nur mit sehr großem Zusatzaufwand berücksichtigt werden [83, 194, 195].

\subsubsection{Anforderungen und Auswahl}

Aus der Analyse der mechanischen Struktur und des Antriebs der Hand sind unter Berücksichtigung der in anderen Händen verwendeten Messverfahren eine Reihe spezifischer Anforderungen an einen Winkelsensor abzuleiten. Sie werden durch die allgemeinen Anforderungen aus dem Abschnitt 2.3.4 ergänzt.

Messbereich und Genauigkeit: Der Sensor soll über einen Winkelbereich von $110^{\circ}\left(90^{\circ}\right.$ zwischen gerade gestrecktem und rechtwinklig gebeugtem Gelenk, zusätzlich an beiden Seiten $10^{\circ}$ Sicherheit) ein möglichst lineares, absolutes Winkelsignal liefern. Der Gelenkwinkel ist auf $\pm 0,5^{\circ}$ genau zu bestimmen, dies entspricht bei einer Fingerlänge von $100 \mathrm{~mm}$ einem Positionsfehler von $\pm 0,87 \mathrm{~mm}$ an der Fingerspitze. Messfehler durch Toleranzen und Spiel in der Fingermechanik sind zu minimieren.

Gelenkinterne Messung: Die flexiblen Fluidaktoren befinden sich direkt in den Fingergelenken. Eine mechanische Übertragungsstrecke wie bei externen Antrieben ist nicht vorhanden. Um eine Übertragungsmechanik für die Messung der Gelenkposition zu vermeiden, ist diese direkt am Gelenk durchzuführen. Dabei ist die Reibung zu minimieren. Zwischen Sensor und Aktor darf kein Scheuern auftreten. Die Sensoren sind vor externen mechanischen Einflüssen, die zu dauerhaften Messfehlern oder der Zerstörung des Sensors führen, zu schützen.

Gelenkintegration: Die Gelenkachse liegt nahe an der Handfläche. Der Sensor ist daher auf den dorsal zur Achse liegenden Bereich zu beschränken. Konzeptionelle Änderungen am Gelenk sind auszuschließen. Die mechanische Stabilität des sensorlosen Gelenks ist nicht oder nur minimal zu verringern.

Kalibrierung: Die Kalibrierdaten für den Sensor sollen einfach aufgenommen werden können. Nach der erstmaligen Inbetriebnahme soll keine Rekalibrierung mehr erforderlich sein. 
Bei der Auswahl des Winkelsensorprinzips werden die externen Sensoren nicht weiter betrachtet, da sie nur mit externen Antrieben sinnvoll einzusetzen sind und eine Übertragungsmechanik für Winkelsensoren alleine nicht sinnvoll erscheint. In der Nähe der Gelenkachsen verlegte Beugesensoren leiden unter den kleinen Biegeradien und erfordern eine regelmäßige Neukalibrierung. Die exponierte Lage auf dem Fingerrücken ist für sie ebenso wenig zu empfehlen, da die Sensoren nicht gegen äußere Einflüsse geschützt sind.

Messprinzipien wie Potentiometer und PSD sind nur bei mittig im Finger angeordneten Gelenkachsen gut einsetzbar. Im gegebenen Fall schränken die palmar liegende Achse und der Aktor im Gelenk den zur Verfügung stehenden Platz so weit ein, dass diese Sensoren nur am Achsende angebracht werden können. Damit sind sie nur schwer vor äußeren mechanischen Einflüssen zu schützen.

Von den zu Beginn dieses Abschnitts beschriebenen möglichen Messverfahren verbleiben somit nur noch aus Magnetfeldsensoren und Permanentmagneten aufgebaute Sensoren. In den folgenden Abschnitten wird diese Messmethode genauer analysiert und auf die Anforderungen des Einsatzes in Fingergelenken mit flexiblen Fluidaktoren optimiert. Dies beinhaltet die Untersuchung verschiedener Varianten für Komponentenanordnung, Sensoren sowie Magnettypen und -geometrien.

\subsubsection{Winkelmessung mit Magnetfeldsensoren}

Dieser Abschnitt analysiert die Eigenschaften verschiedener Magnetfeldsensoren und betrachtet die zu erwartenden Signalverläufe verschiedener Anordnungen mit Permanentmagneten.

\section{Magnetfeldsensoren}

Die am weitesten verbreiteten Halbleitersensoren zur Erfassung von Magnetfeldern nutzen den Halleffekt, den anisotropen magnetoresistiven Effekt (AMR) oder den Riesen-Magnetowiderstandseffekt (giant magnetoresistive effect, GMR). Beim Halleffekt (Abbildung 4.1 links) wird an einem stromdurchflossenen Leiter normal zur Richtung des Stromes $I$ und der Richtung der magnetischen Flussdichte $B$ die Hall-Spannung $U$ gemessen. Bei den magnetoresistiven Effekten (Abbildung 4.1 rechts) ändert sich der Widerstand $R$ des stromdurchflossenen Sensors in Richtung der magnetischen Flussdichte. Durch Anordnung als Wheatstone-Brücke wird bei diesen Sensoren ein Spannungssignal erzeugt.

AMR- und GMR-Sensoren sind mit Messbereichen von etwa $\pm 1 m T$ bis $\pm 10 \mathrm{mT}$ sehr empfindlich und können bei einem Erdmagnetfeld von ca. $\pm 50 \mu T$ auch als elektronischer Kompass verwendet werden. Hallsensoren sind mit einem Messbereich von bis zu etwa $\pm 100 m T$ um eine bis zwei Größenordnungen weniger empfindlich [151].

Um die Messfehler durch von außen wirkende Magnetfelder möglichst klein zu halten, wird die weitere Betrachtung auf Hallsensoren ausgerichtet. Der zu erwartende maximale Messfehler durch das Erdmagnetfeld bei einem Messbereich 

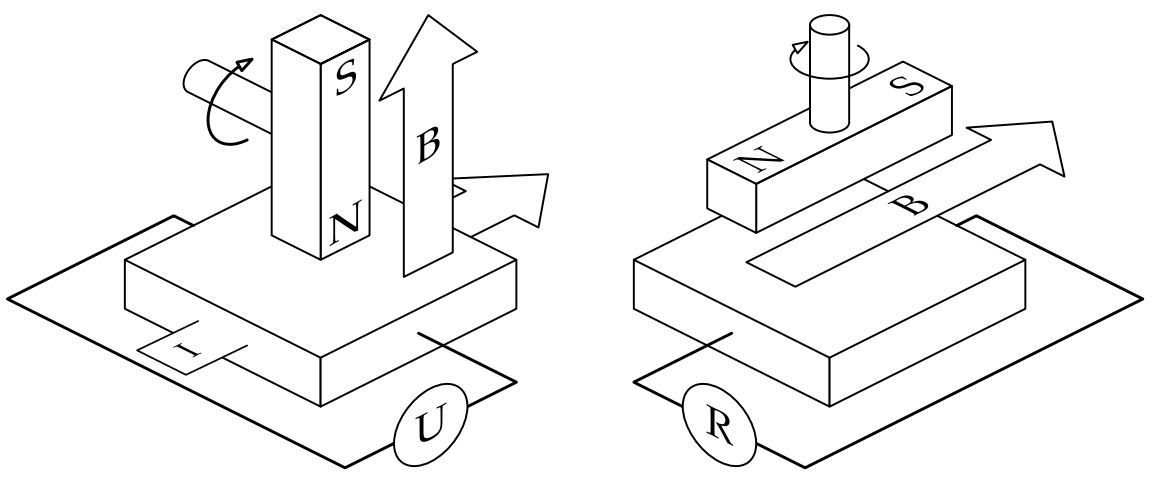

Abbildung 4.1: Prinzip: Hallsensor (links), magnetoresistiver Sensor (rechts).

von $\pm 100 \mathrm{mT}$ ist mit etwa $\pm 50 \mu T \cdot \frac{110^{\circ}}{200 \mathrm{mT}}= \pm 0,028^{\circ}$ abzuschätzen. Er liegt damit bei etwa $5 \%$ des zulässigen Messfehlers.

Ebenfalls für Hallsensoren spricht die Verfügbarkeit von integrierten Sensoren, die nicht nur ein Hallelement, sondern auch Stromregler, Signalverstärker und Temperaturkompensation beinhalten [7]. Das Ausgangssignal $U_{\mathrm{A}}$ dieser Sensoren in Gleichung (4.1) ist innerhalb des Messbereichs $\left(B_{\mathrm{n}} \leq \pm 100 \mathrm{mT}\right)$ ratiometrisch, d.h. Nullpunkt und Verstärkung sind proportional zur Versorgungsspannung $U_{\mathrm{V}}$. Damit können bei Verwendung der Versorgungsspannung als Referenzspannung des A/D-Wandlers Fehler durch Störungen in der Versorgungsspannung minimiert werden.

$$
U_{\mathrm{A}}=\frac{U_{\mathrm{V}}}{2}\left(1+\frac{B_{\mathrm{n}}}{200 m T}\right)
$$

\section{Anordnung}

Verschiedene Anordnungen von Magneten und Hallsensoren zur Winkelmessung wurden bereits in [153] mit der 2D-FEM-Magnetsimulation FEMM [126] untersucht. Varianten, bei denen ein Stabmagnet mit einem seiner Pole auf einen Sensor zu bewegt wird, nutzen nur die Hälfte des Messbereichs und weisen starke Nichtlinearitäten auf (Abbildung 4.2, Fall 1). Werden Sensor und Magnet aneinander vorbei bewegt, kann der gesamte Messbereich ausgenutzt werden. Das Ausgangssignal ist jedoch bestenfalls auf der Länge des Magneten gut linearisierbar (Abbildung 4.2, Fall 2). Dies gilt jedoch nur für kurze Stabmagnete, was eine entsprechende Mechanik zu Umsetzung der Gelenkrotation in eine Translation erfordert.

Bei einem Scheiben- oder Ringmagnet mit diametraler Magnetisierung ist der Betrag der magnetischen Flussdichte in der Mittelebene von der Entfernung und der Richtung der Feldlinien proportional vom Winkel abhängig. Gleiches gilt auch für Ringmagnete, die magnetische Flussdichte ist dabei geringer. Mit einem Hallsensor, der nur die Normalkomponente $B_{\mathrm{n}}$ der magnetischen Flussdichte $B_{0}$ er- 

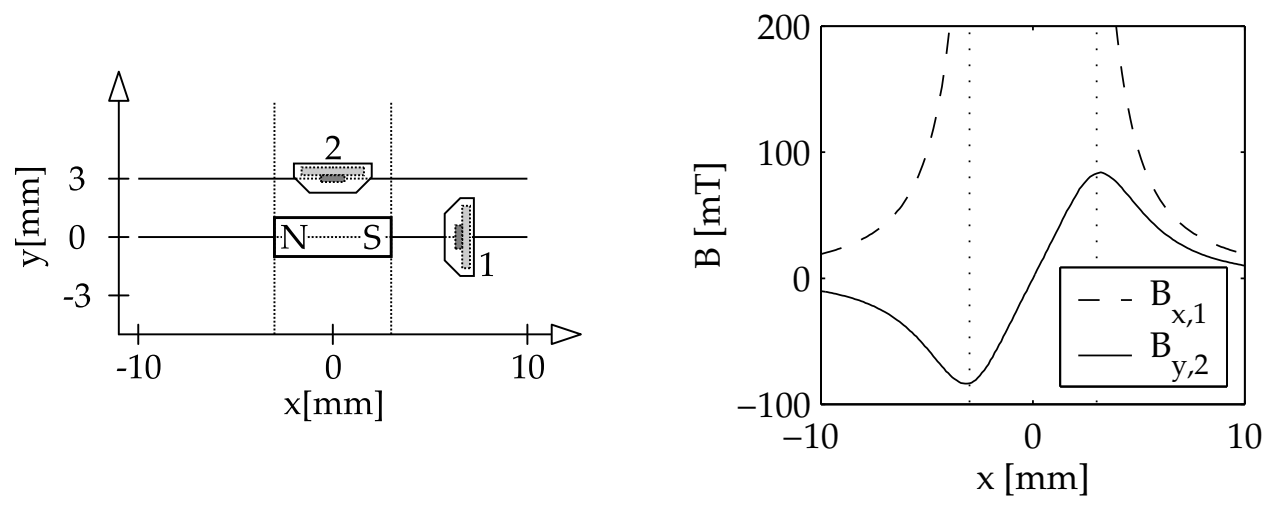

Abbildung 4.2: Lineare Bewegung von Hallsensoren relativ zu einem Stabmagneten. Fall 1: Annäherung an eine Polseite, der Hallsensor erfasst $B_{\mathrm{x}}$; Fall 2: Bewegung parallel zur Polachse, gemessen wird $B_{\mathrm{y}}$. Links: Anordnung. Rechts: Sensorsignal.

fasst, ergibt sich ein sinusförmiges Signal (Abbildung 4.3 rechts).

$$
B_{\mathrm{n}}=B_{0} \cdot \sin \left(\varphi-90^{\circ}\right)
$$
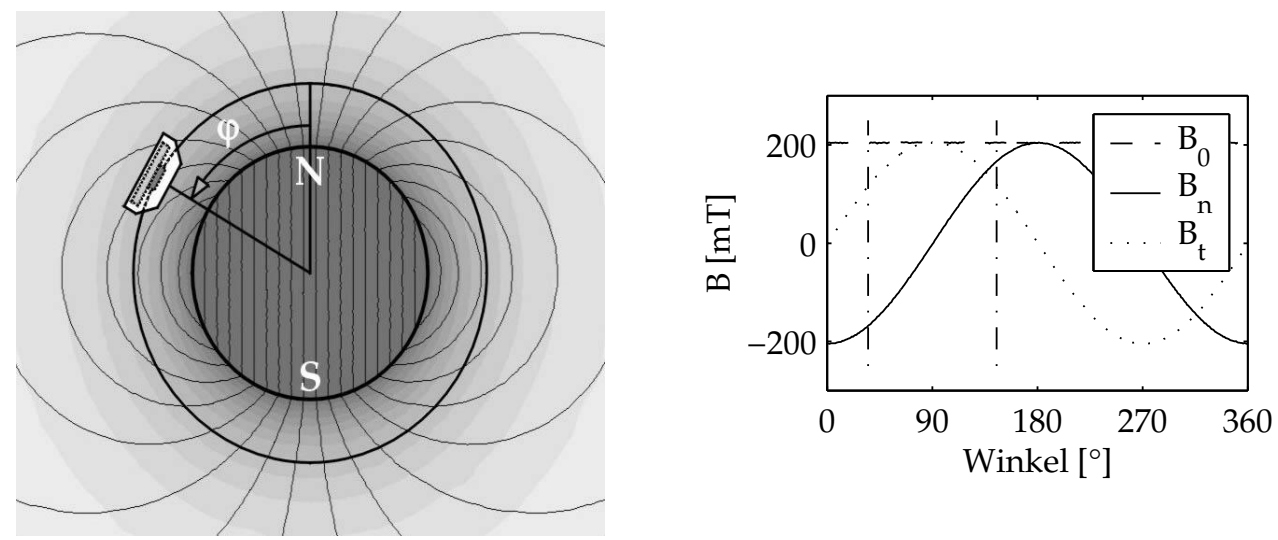

Abbildung 4.3: Rotation eines Hallsensors um einen Scheibenmagneten mit diametraler Magnetisierung. Links: Anordnung. Rechts: Signalverläufe der magnetischen Flussdichten ( $B_{0}$ absolut, $B_{\mathrm{n}}$ normal, $B_{\mathrm{t}}$ tangential).

Für die absolute Messung der Gelenkposition im geforderten Bereich von $110^{\circ}$ ist daher ein einzelner Hallsensor ausreichend. Sensor und Magnet sind so zueinander anzuordnen, dass bei mittlerer Gelenkstellung $\left(\varphi=45^{\circ}\right) B_{\mathrm{n}}=0$ ist. Hierfür ist der Magnet um $45^{\circ}$ entgegen der Beugerichtung des Gelenks zu drehen, so dass sich das Sensorsignal aus Gleichung (4.2) zu Gleichung (4.3) ändert.

$$
B_{\mathrm{n}}=B_{0} \cdot \sin \left(\varphi-45^{\circ}\right) \text {. }
$$


Da die dem Sensor abgewandte Seite des Magneten im Messbereich nur wenig zur magnetischen Flussdichte beiträgt, kann der Magnet auch auf Kosten der Signallinearität und -amplitude halbiert werden. Durch diese Maßnahme wird das Gelenkinnenteil weniger als bisher geschwächt. Gleichzeitig wird die magnetische Flussdichte auf der Innenseite der Hand reduziert.

\section{Kalibrierung und Signalapproximation}

Zur Ermittlung der Winkelstellung aus dem Sensorsignal ist die Kalibrierung des Sensorsystems erforderlich. Steht für die definierte Positionierung des Gelenks über den gesamten Bewegungsbereich ein Teststand zur Verfügung, können Kalibrierdaten aufgenommen werden und mit der Methode der kleinsten Fehlerquadrate die Polynomkoeffizienten $w_{i}$ für Gleichung (4.4) bestimmt werden.

$$
\hat{\varphi}=\sum_{i=1}^{n}\left(w_{i} \cdot B_{\mathrm{n}}^{i}\right)
$$

$\mathrm{Zu}$ den Signalverläufen aus der Simulation von Ring- und Halbringmagneten (Abbildung 4.4 links) wurden mit Hilfe eines Matlab-Programms die in Abbildung 4.4 gezeigten maximalen und mittleren Approximationsfehler für unterschiedliche Polynomgrade ermittelt.

Am linken Rand des Fehlerdiagramms sind die Messfehler für eine vereinfachte Kalibrierung dargestellt, bei der die Winkelstellung durch lineare Interpolation zwischen den an den Endanschlägen ermittelten Signalwerten berechnet wird. Dies reduziert nicht nur den Rechenaufwand gegenüber Polynomen höherer Ordnung, sondern ermöglicht bei Ergänzung der Steuersoftware auch eine sehr einfache manuelle oder teilautomatisierte Kalibrierung der kompletten Hand. Kann durch die Aktoren die Bewegung der Gelenke bis an die Endanschläge sichergestellt werden, ist der Kalibriervorgang vollständig automatisierbar, indem bei der ersten Inbetriebnahme einer Hand nacheinander mit allen Gelenken der gesamte Arbeitsbereich abgefahren wird.

Für beide Magnetformen wird ab einem Polynom dritter Ordnung ein mittlerer Fehlerbetrag $\delta$ von $0,5^{\circ}$ unterschritten. Die Kalibrierung durch Vermessung an den Endanschlägen alleine liefert erwartungsgemäß die größten Fehler, mit $4^{\circ}$ für den Ring und $6^{\circ}$ beim Halbring liegt der maximale Fehler deutlich über den Zielwerten. Die Approximation des Signalverlaufes mit Hilfe einer Gerade und einer Exponentialfunktion nach Gleichung (4.5) liefert auch bei Teilung in zwei Abschnitte $\left(B_{\mathrm{n}}<0 ; B_{\mathrm{n}} \geq 0\right)$ einen mittleren Fehlerbetrag von $0,58^{\circ}$ und einen Maximalfehler von $2,18^{\circ}$. Das Ergebnis ist damit bei erhöhtem Aufwand in der Online-Berechnung etwas schlechter als das Polynom dritter Ordnung.

$$
\hat{\varphi}=w_{0}+w_{1} \cdot B_{\mathrm{n}}+w_{2} \cdot e^{B_{\mathrm{n}}}
$$

\subsubsection{Mechanische Integration}

Das im vorangegangenen Abschnitt hergeleitete Prinzip lässt sich auf mehrere Weisen in ein Gelenk (Abbildung 4.5 links) integrieren. 

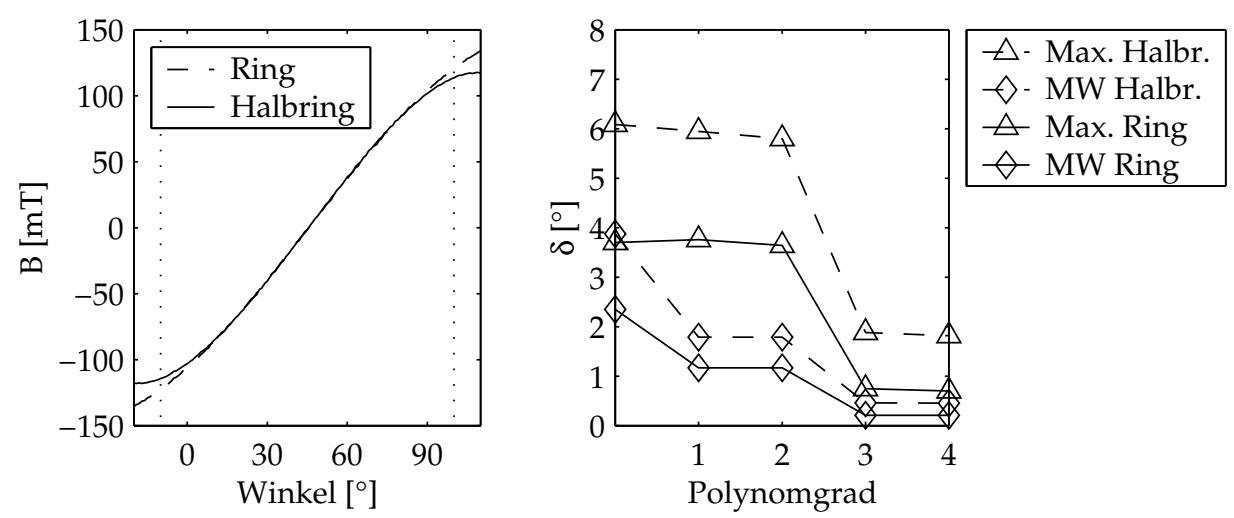

Abbildung 4.4: Links: FEM-Simulation des Sensorsignals für Ring- und Halbringmagnet. Rechts: maximaler (Max.) und mittlerer (MW) Approximationsfehler in Abhängigkeit vom Polynomgrad der Gleichung (4.4).

\section{Sensor in Gabelvertiefung}

Die in [153] vorgeschlagene Variante schlägt eine Vertiefung der Gabel für den Hallsensor und eine Schlitzung des Gelenkinnenteils für den Magneten vor (Abbildung 4.5 rechts). Diese wirkt sich auf die Stabilität des Gelenks sehr nachteilig aus [66].
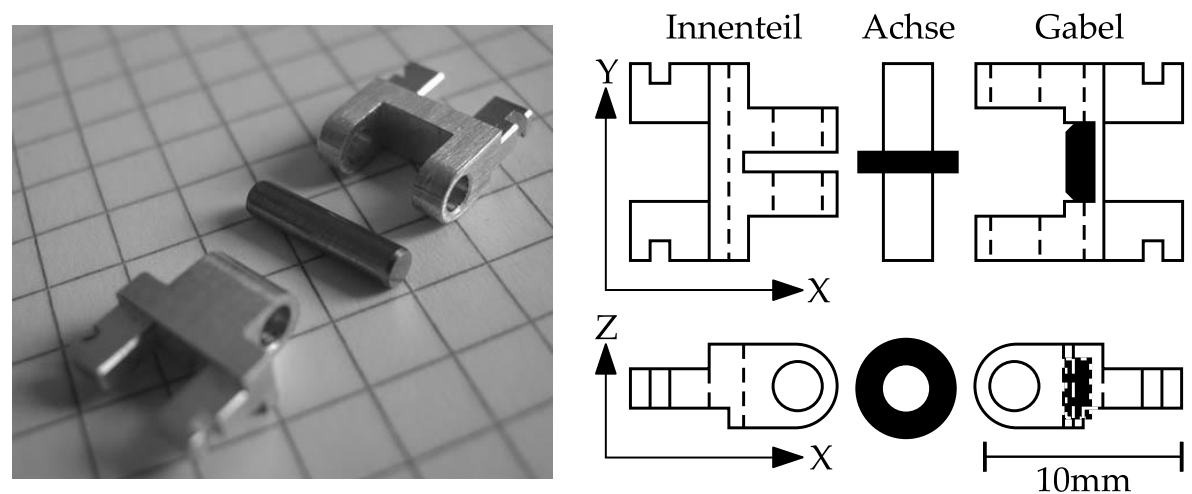

Abbildung 4.5: Links: Einzelteile des Fingergelenks (Innenteil, Achse und Gabel). Rechts: Integrationsvorschlag nach [153].

\section{Sensor an Gelenkaußenseite}

Die Anordnung des Sensors an der Gelenkaußenseite (Abbildung 4.6) ist ebenfalls nicht zu empfehlen, da der Sensor sehr exponiert angebracht ist und damit einem erhöhten Risiko mechanischer Beschädigung ausgesetzt ist. Bei dieser Anordnung wirken sich die aus dem Gelenkaufbau resultierenden Toleranzen (Abbil- 
dung 4.7) besonders stark aus. Die Achse ist in die untere Gabelhälfte eingepresst, zum Innenteil und der oberen Gabelhälfte liegen Spielpassungen vor. Zusätzlich kann sich das Innenteil in der Gabel entlang der Achse bewegen. Als Messfehler wirkt sich bei Querbelastungen die Abstandsänderung zwischen Hallsensor und Magnet des Fingers besonders stark aus.

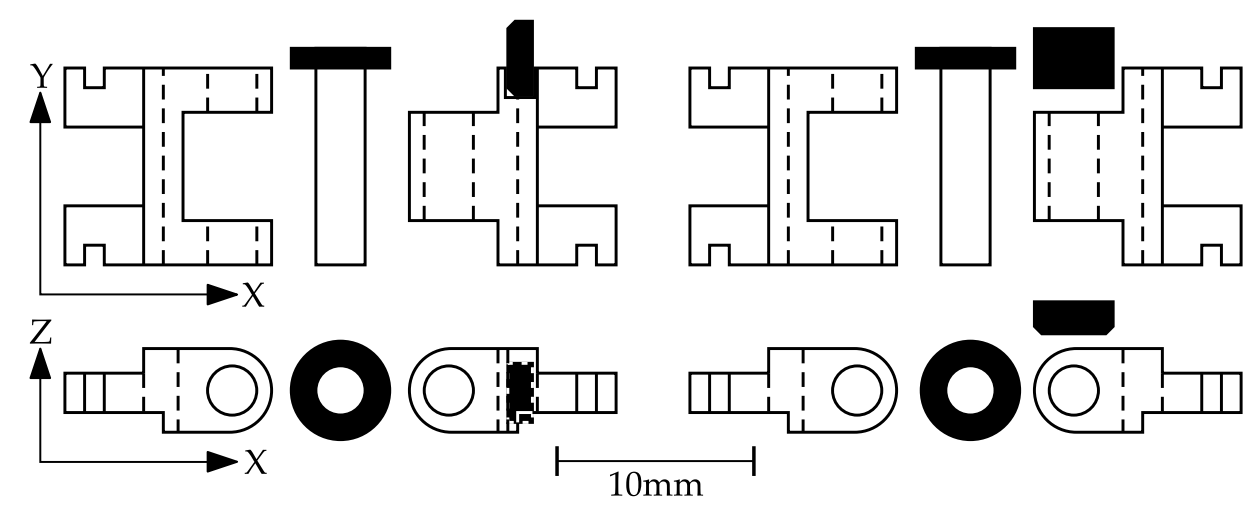

Abbildung 4.6: Sensoranordnungen an der Gelenkaußenseite. Links: Sensor neben Magnet; Rechts: Sensor über Magnet.

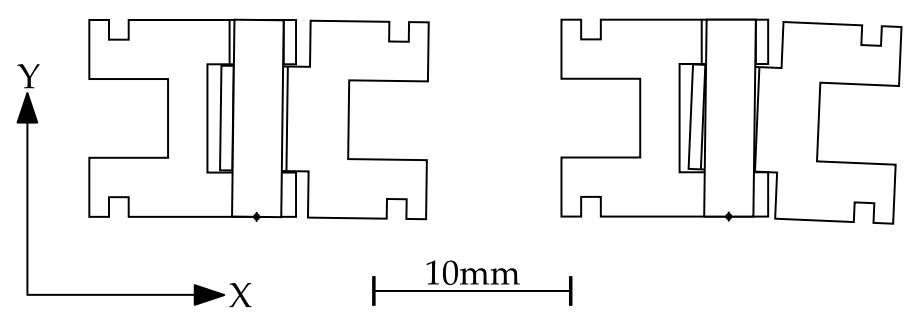

Abbildung 4.7: Schnittdarstellung der Toleranzen des Fingergelenks, die Überund Untermaße sind überhöht dargestellt. Links: Zugbelastung, Rechts: Querbelastung.

\section{Sensor dorsal der Achsmitte}

$\mathrm{Zu}$ bevorzugen ist eine achsmittige Anordnung von Magnet und Sensor wie in Abbildung 4.8 links. Der Hallsensor befindet sich dabei auf der Aktorseite des Gelenks. Der Zusammenbau des Gelenks wird durch einen Halbringmagneten (Abbildung 4.8 rechts) vereinfacht, da er nachträglich eingesetzt werden kann und durch die Form der Nut der bei Ringmagneten erforderliche Ausrichtvorgang entfällt. 


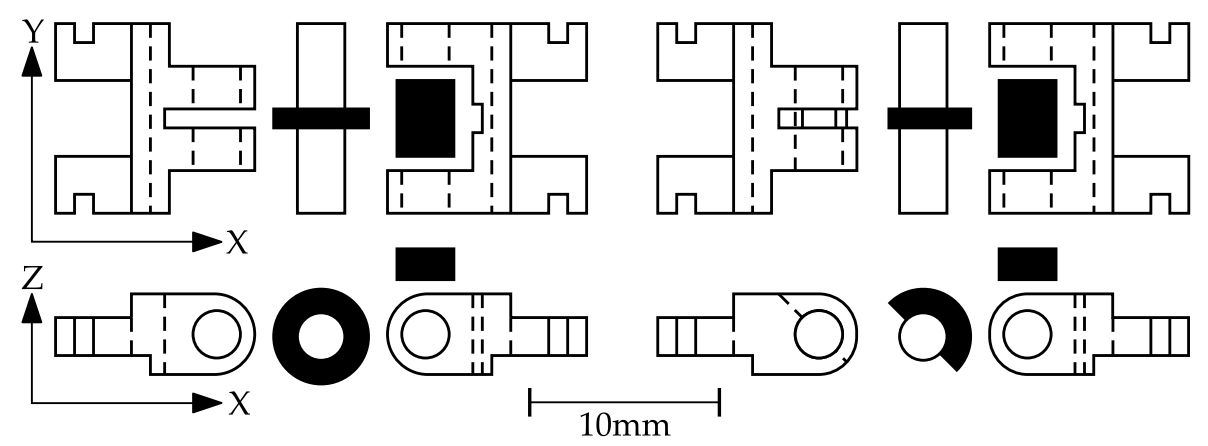

Abbildung 4.8: Achsmittige Sensoranordnungen. Links: Ringmagnet. Rechts: Halbringmagnet.

\section{Positionierung des Hallsensors}

Für die horizontale Anbringung des Hallsensors (Daten siehe Anhang, Abschnitt 7.1.1) wie in Abbildung 4.8 bietet sich eine Leiterplatte auf der Rückenseite der Fingerstruktur mit einer Aussparung für das Aktorblech an. Durch die außermittige Positionierung des Hallelements im Gehäuse lassen sich mit zwei möglichen Abständen zum Magneten unterschiedliche Signalamplituden realisieren. Abbildung 4.9 zeigt die Anordnung für eine Leiterplattendicke von 1,5 $\mathrm{mm}$, einen Magnetdurchmesser von $5 \mathrm{~mm}$ und eine Dicke der Fingerstruktur von $4 \mathrm{~mm}$, der Magnet steht also 0,5 $\mathrm{mm}$ aus dem Gelenkkörper über.

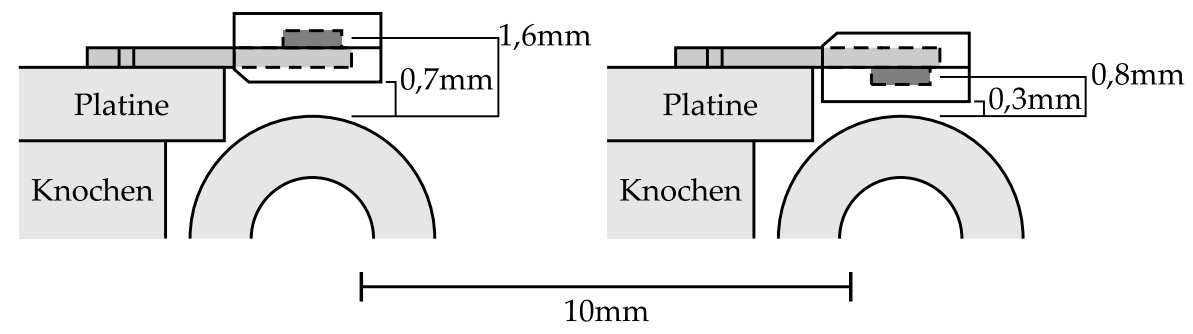

Abbildung 4.9: Anbringungsvarianten für einen liegenden Hallsensor an einer Leiterplatte. Links ist die beschriftete Vorderseite vom Magneten abgewandt, rechts dem Magneten zugewandt.

Die rechts dargestellte Anordnung ist Platz sparender und liefert durch den geringeren Sensorabstand eine größere Signalamplitude.

\subsubsection{Dimensionierung}

Aus der vorangegangenen Betrachtung verbleiben für die Dimensionierung des Magneten zunächst die Faktoren Material und Außendurchmesser. Der darauf folgende Abschnitt betrachtet die weitere Optimierung der Magnetgeometrie. 


\section{Magnettypen}

Neben den sehr weit verbreiteten Aluminium-Nickel-Kobalt-Magneten (AlNiCo) und Ferritmagneten (Barium- und Strontiumferrit) werden immer häufiger Seltenerdmagnete aus Neodym-Eisen-Bor (NdFeB) und Samarium-Kobalt (SmCo) eingesetzt. Sie liefern in der genannten Reihenfolge bei gleicher Bauform stärkere Magnetfelder. Für gesinterte Ferritmagnete spricht ihre preiswerte Herstellung in großen Stückzahlen und ihre hohe Korrosionsbeständigkeit. AlNiCo-Magnete, die auch gegossen werden können, sind relativ leicht zu demagnetisieren.

Gesinterte Seltenerdmagnete sind noch spröder, leichter splitternd und weniger korrosionsfest als AlNiCo-Magnete. Sie zeichnen sich jedoch durch eine höhere Leistungsfähigkeit aus. Unter Verzicht auf einen Teil dieser Leistungsfähigkeit können durch Vermischen mit Kunststoff im Spritzgussverfahren sehr komplizierte Formen mit hoher Formgenauigkeit hergestellt werden, was insbesondere bei großen Stückzahlen interessant ist. Die Korrosionsfestigkeit wird typischerweise durch Beschichtung mit Nickel, Zink oder Zinn erhöht.

\section{Magnetdurchmesser}

Für den Aufbau aus Abbildung 4.9 ergibt sich bei unterschiedlichen Magnettypen für Ringmagneten die in Abbildung 4.10 dargestellte Entfernungsabhängigkeit. AlNiCo und Ferrit sind bei einer gewünschten Signalamplitude von $100 \mathrm{mT}$ nicht ausreichend. Die kunststoffgebundenen NdFeB10-Magnete weisen eine gut ausreichende Amplitude auf. Sie sind daher für den Einsatz bei großen Stückzahlen interessant. Für die später aufgebauten Prototypen wird der Typ ME30 NdFeB verwendet.

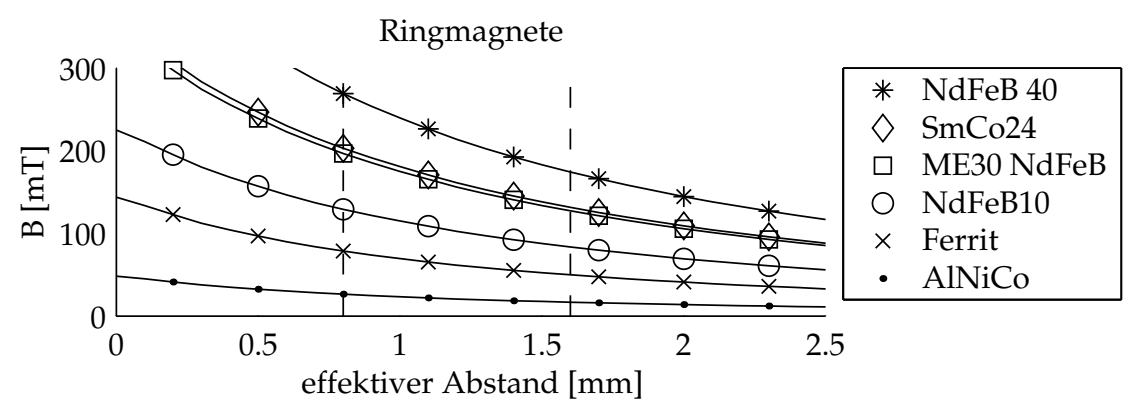

Abbildung 4.10: Entfernungsabhängigkeit der magnetischen Flussdichte unterschiedlicher Materialien für Ringmagnete nach Abbildung 4.9 .

Aus Abbildung 4.11 geht die bei Halbringen in den Endanschlägen des Gelenks zu erwartende geringere Amplitude hervor, der relative Unterschied bei gleichem Magnettyp vergrößert sich mit steigendem Abstand von etwa $8 \%$ an der Magnetoberfläche auf $46 \%$ bei 2,5 $\mathrm{mm}$ Abstand.

Die Auswahl des Magnettyps für den Halbringmagnet führt zu den gleichen Typen wie beim Ringmagneten. 


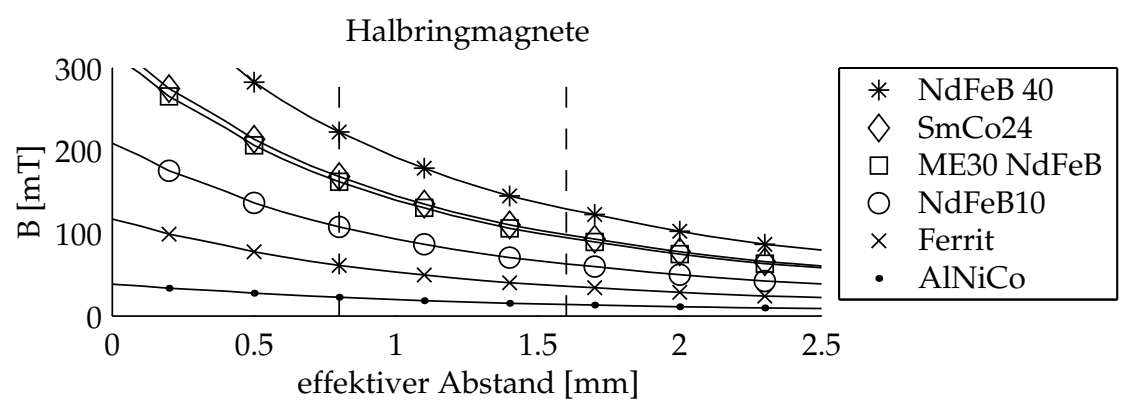

Abbildung 4.11: Entfernungsabhängigkeit der magnetischen Flussdichte unterschiedlicher Materialien für Halbringmagnete nach Abbildung 4.9 .

\subsubsection{Geometrieoptimierung des Magneten}

Mit der halbkreisförmigen Magnetkontur lässt sich bereits eine ausreichende Funktionalität erreichen, die jedoch wegen der Nichtlinearität in den Endlagen des Gelenks das Kriterium der einfachen Kalibrierung noch nicht erfüllt. Zum Erreichen dieses Ziels wurde die Magnetgeometrie auf eine höhere Signallinearität optimiert. Dabei werden als Randbedingung Magnetsymmetrie, Richtung der Magnetisierung, Minimal- und Maximaldurchmesser fest vorgegeben [124].

\section{Genetischer Algorithmus}

Die zu lösende Optimierungsaufgabe stellt an der Außenseite und in der Mitte des Magneten besondere Herausforderungen an den Optimierungsalgorithmus: Die vom Hallsensor nicht überstrichenen Außenbereiche des Magneten beeinflussen den Signalverlauf im Messbereich, umgekehrt dazu kann in der Magnetmitte mit einem beliebigen Magnetdurchmesser das gewünschte Ergebnis erzielt werden.

Als Optimierungsverfahren wurde ein genetischer Algorithmus gewählt und die in [67] vorgestellte Variante in Matlab implementiert (Code siehe Abschnitt 7.3 im Anhang) und für die Optimierungsaufgabe modifiziert. Der genetische Algorithmus ist als steady-state mit Turnierselektion ausgeführt. Auf Nachbarschaftsbeziehungen in der Population mit 25 Genotypen wird dabei verzichtet.

\section{Kodierung des Genotyps}

Der Genotyp beschreibt die Magnetaußenkontur an Stützpunkten $g_{i}$ mit einem Abstand von $10^{\circ}$. Alle Stützpunkte außer $g_{n}$ werden doppelt verwendet (Abbildung 4.12). Durch diese Maßnahme wird eine symmetrische Magnetkontur sichergestellt.

Zur Erreichung einer gleichmäßigen Außenkontur werden weitere Zwischenpunkte nach Gleichung (4.6) ähnlich einem einschichtigen neuronalen Netzwerk radialer Basisfunktionen (RBF) mit einem fest vorgegebenen Weiteparameter $\sigma$ be- 


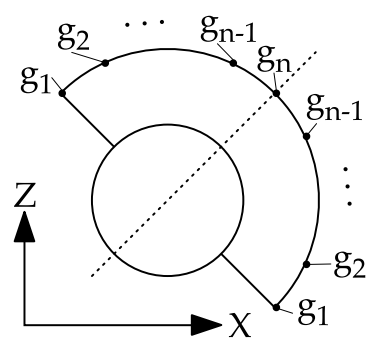

Abbildung 4.12: Kodierung der Magnetkontur im Genotyp.

rechnet.

$$
r(\varphi)=r_{0}+\sum_{i=1}^{n} g_{i} \cdot e^{\frac{\left(\beta_{i}-\varphi\right)^{2}}{(2 \cdot \sigma)^{2}}}
$$

Die Radien der Magnetkontur werden in $1^{\circ}$-Schritten ermittelt und unter Berücksichtigung der Minimal- und Maximalradien kartesische Koordinatenpunkte umgerechnet. Diese werden in ein Skript zur Erzeugung der Magnetkontur und der Durchführung der FEM-Simulation geschrieben. Als Ergebnis wird die normale Flussdichte entlang der vorgesehenen Sensortrajektorie geliefert.

\section{Fitnessfunktion}

Die Qualität $f$ einer Lösung wird in Gleichung (4.7) als eine gewichtete Summe mehrerer Fitnessfunktionen bestimmt, die die Signallinearität $f_{1}$, die Ausnutzung des Sensormessbereichs $f_{2}$ und die Mindestmaterialstärke $f_{3}$ des Magneten bewerten. Die Gewichtung in der Fitnessfunktion $f$ erfolgte an Hand der empirischen Bewertung der Startpopulation.

$$
f=5 \cdot f_{1}+f_{2}+f_{3}
$$

Aus dem Fehler bei der Approximation des Sensorsignals an die Gleichung (4.8) nach der Methode der kleinsten Fehlerquadrate wird in Gleichung (4.9) das Linearitätsmaß $f_{1}$ ermittelt.

$$
\hat{\varphi}=m \cdot B_{\mathrm{n}}+b
$$

Für die Steigung $m$ wird ein zulässiger Bereich von $m_{\min }$ bis $m_{\max }$ definiert. Die Obergrenze $m_{\max }$ sichert, dass der Sensor in den Extremlagen der Gelenke nicht übersteuert wird, während durch die Untergrenze $m_{\min }$ eine Mindestausnutzung des Sensormessbereichs erreicht wird. Das Unter- oder Überschreiten der geforderten Grenzen bewirkt eine Abwertung der Lösung über den Strafterm $f_{2}$ in Gleichung (4.10); auf gleiche Weise wirkt $f_{3}$ der Gleichung (4.11) beim Unterschreiten des geforderten Mindestradius $r_{\text {min }}$. 


$$
\begin{aligned}
& f_{1}=-\log \left(1+\sqrt{\sum_{i=1}^{n}\left(\varphi_{i}-\hat{\varphi}_{i}\right)^{2}}\right) \\
& f_{2}=\left\{\begin{aligned}
-\log \left(1+\left(m_{\min }-m\right)^{2}\right) & : m<m_{\min } \\
0 & : m_{\min } \leq m \leq m_{\max } \\
-\log \left(1+\left(m_{\max }-m\right)^{2}\right) & : \quad m>m_{\max }
\end{aligned}\right. \\
& f_{3}=\left\{\begin{aligned}
0 & : \min (r) \geq r_{\text {min }} \\
-\log \left(1+\left(r_{\text {min }}-\min (r)\right)^{2}\right) & : \min (r)<r_{\text {min }}
\end{aligned}\right.
\end{aligned}
$$

\section{Initialisierung der Population}

Das A-Priori-Wissen über die Einsetzbarkeit halbkreisförmiger Magnete wird bei der Initialisierung der Population anstatt der meist üblichen Zufallsinitialisierung genutzt. Die Radien der Startpopulation sind etwas über den Bereich zulässiger Radien hinaus gleichverteilt angeordnet. Hierdurch werden nicht nur bereits relativ gute Startlösungen, sondern auch regelmäßige Formen vorgegeben, deren Diskretisierung für die FEM-Analyse im Normalfall unproblematisch ist.

\section{Mutations- und Rekombinationsoperatoren}

Die Turnierselektion stellt sicher, dass der Gewinner eines Vergleiches immer unverändert erhalten bleibt. Mutation und Rekombination werden nur auf den Verlierer angewendet.

Da die Population mehrheitlich mit zu großen Magnetdurchmessern initialisiert wird, ist die Mutation auf die Erzeugung kleinerer Magnete ausgerichtet. Die Änderung der per Zufall ausgewählten Gene wird daher mittels im Intervall $-0.2 \mathrm{~mm}<\Delta g<+0.1 \mathrm{~mm}$ gleichverteilter Zufallszahlen ermittelt.

Bei der Rekombination wird ein Abschnitt der Gene des Gewinners auf den Verlierer kopiert, Position und Länge werden per Zufall bestimmt. Dabei werden maximal $30 \%$ der Gene überschrieben.

\section{Ergebnis}

Der beschriebene genetische Algorithmus liefert den in Abbildung 4.1.7 links gezeigten Radienverlauf des Magneten, aus der die Magnetkontur berechnet werden kann (Abbildung 4.1.7, rechts). Der Magnetradius reicht im Außenbereich nahe an den zulässigen Maximalradius heran und nimmt zur Mitte hin auf das zulässige Minimum ab.

Der Bereich der zu erwartenden magnetische Flussdichte nutzt den Messbereich des Hallsensors vollständig aus (Abbildung 4.14, links). Unmittelbar jenseits der beiden mechanischen Begrenzungen des Gelenks wird die maximale Flussdichte des Hallsensors erreicht. Innerhalb des geforderten Winkelmessbereichs ist der in Abbildung 4.14 rechts dargestellte Verlauf des Positionsfehlers zu erwarten. So beträgt der maximale Fehlerbetrag bei linearer Interpolation zwischen den 

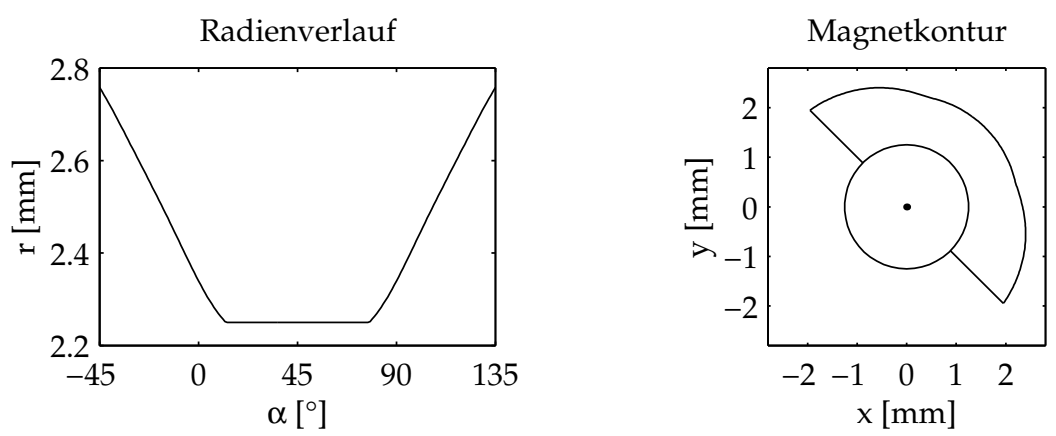

Abbildung 4.13: Radienverlauf (links) und Kontur (rechts) des optimierten Magneten.

Endwerten $0,4^{\circ}$, der mittlere Fehlerbetrag liegt bei $0,3^{\circ}$. Damit kann die geforderte Genauigkeit auch ohne die aufwändige Aufnahme einer Kalibrierkennlinie in einer Testvorrichtung erreicht werden.
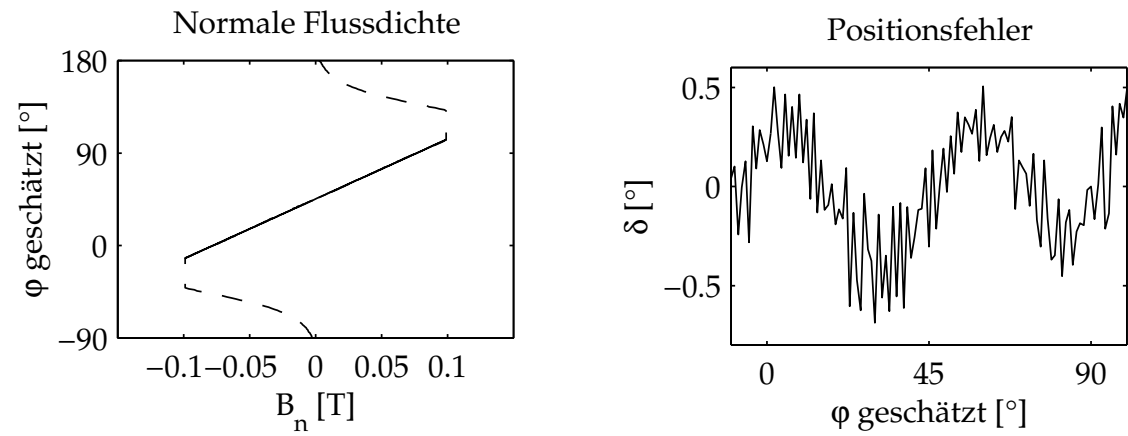

Abbildung 4.14: Signalverlauf der normale Flussdichte (links) und des Positionsfehlers (rechts) beim optimierten Magneten.

\subsubsection{Aufbau und Test von Prototypen}

Für Tests wurde eine Reihe von Prototypen in unterschiedlichen Konfigurationen (Tabelle 4.1) entwickelt. Sie umfassen die vier bereits diskutierten Sensoranordnungen, bei denen zusätzlich die Ausrichtung der Hallsensoren variiert wird.

Die Prototypen (Abbildung 4.15) bestehen aus modifizierten Gelenken und Fingergliedern, an denen Sensorplatine und Magnet (Daten siehe Anhang, Abschnitt 7.1.2) angebracht sind. Auf der Platinenseite befindet sich ein Aktorblech zur Führung der Platine, auf den Rest des Gelenkantriebs wird verzichtet.

Messungen wurden mit einem Aktorteststand (Abbildung 4.16) durchgeführt, in dem das Gelenk durch einen getriebeuntersetzten Schrittmotor positioniert wird [11]. Über den Bewegungsbereich des Gelenks wird das Sensorsignal in $1^{\circ}$ Schritten von einem Messrechner aufgezeichnet. Die über zehn Messungen gemittelten Signalverläufe (Abbildung 4.17) zeigen, dass die Varianten mit vom Magne- 


\begin{tabular}{l|c|c|c|c|c|} 
Variante & \multicolumn{4}{|c|}{ Magnet } & \multicolumn{2}{c|}{ Hallsensor } \\
& Typ & Anordnung & Befestigung & Ausrichtung & Anordnung \\
\hline A1 & Ring & mittig & Innenteil & hor. oben & abgewandt \\
A2 & Ring & mittig & Innenteil & hor. oben & zugewandt \\
B1 & Halbring & mittig & Innenteil & hor. oben & abgewandt \\
B2 & Halbring & mittig & Innenteil & hor. oben & zugewandt \\
C2 & Ring & außen & Gabel & vert. seitlich & zugewandt \\
D1 & Ring & außen & Gabel & hor. oben & abgewandt \\
D2 & Ring & außen & Gabel & hor. oben & zugewandt \\
\hline
\end{tabular}

Tabelle 4.1: Konfigurationen der Winkelsensorprototypen. Der Buchstabe kennzeichnet die Variante, 1 einen vom Magneten abgewandten Sensor, 2 einen dem Magneten zugewandten Sensor (hor.=horizontal; vert.=vertikal).

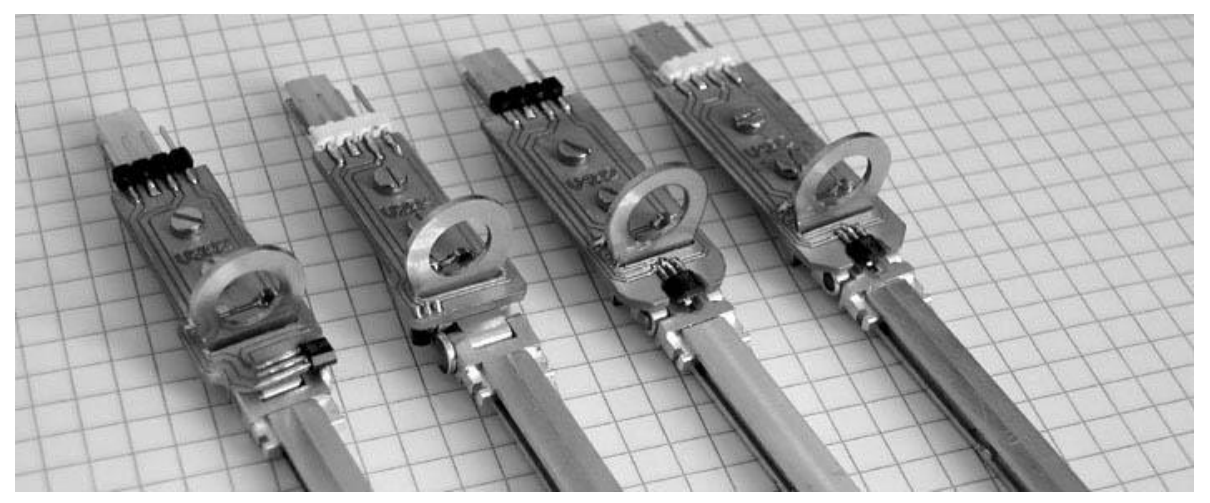

Abbildung 4.15: Prototypen der Varianten A1, B1, C2 und D1 (v.l.n.r). 
ten abgewandten Sensoren den Messbereich des Hallsensors nur teilweise ausnutzen; die anderen Varianten nutzen den Signalbereich sehr gut aus.

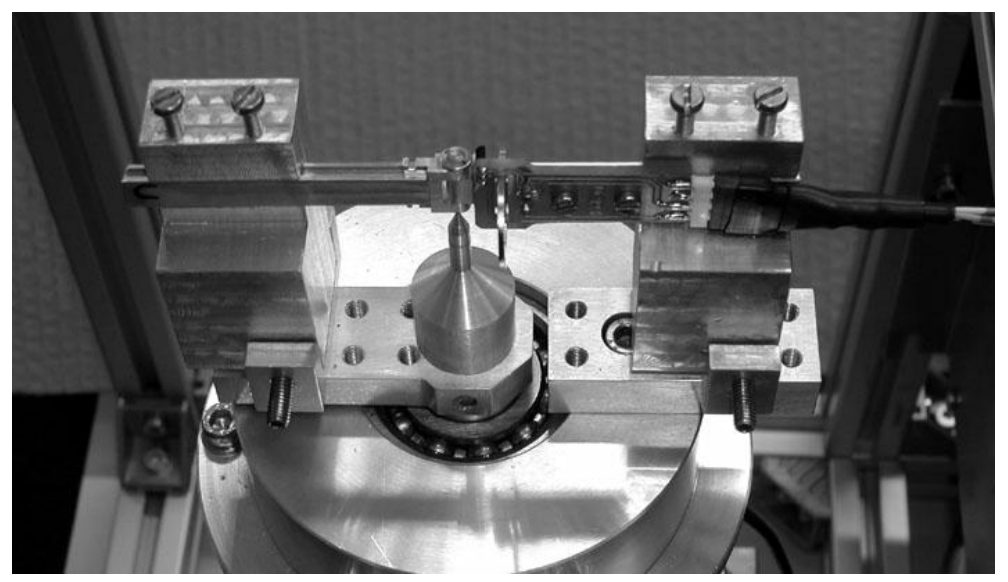

Abbildung 4.16: Aktorteststand zur Vermessung der Sensoren.

Die Sensorkennlinie wird aus den gemittelten Sensormessdaten nach der Methode der kleinsten Fehlerquadrate berechnet. Abbildung 4.18 zeigt die Standardabweichung der Messwerte gegenüber der approximierten Sensorkennlinie; außer beim Sensor A1 liegen die Werte unterhalb von $0,28^{\circ}$.

\subsection{Taktile Sensoren}

Der Einsatz taktiler Sensoren in künstlichen Händen und Greifern dient beim Zugreifen der Erkennung des Objektkontakts und der Erfassung der auf das Objekt ausgeübten Kräfte. So kann mit einer Kraftregelung sichergestellt werden, dass für das sichere Greifen ausreichende Kräfte ausgeübt werden. Die Beschädigung eines Objekts durch zu hohe Greifkräfte kann ebenfalls verhindert werden. Ferner kann in Phasen ohne gewünschten Objektkontakt eine Kollisionsüberwachung realisiert werden.

Die Nachbildung des menschlichen Tastsinns mittels taktiler Sensoren gilt als eine der großen Herausforderungen bei der Weiterentwicklung künstlicher Hände. Besonders schwierig zu lösen ist der Konflikt zwischen dem Ziel einer möglichst genauen Kraftmessung und dem Wunsch nach dem Einsatz eines elastischen Fingergewebes. Letzteres ist für das sichere Greifen sehr vorteilhaft, da bereits bei geringen Kontaktkräften unabhängig von der Oberflächengeometrie eine große Kontaktfläche erreicht wird [36, 90, 91, 92].

Bei der Auswahl des Messprinzips und der Realisierung der Sensoren sind als Hauptkriterien sowohl die von der Hand zu übernehmenden Aufgaben als auch die verwendete Antriebstechnik zu berücksichtigen.

Eine umfassende Darstellung des Standes der Technik taktiler Sensortechnologien der Robotik in den 80er Jahren ist in [196] gegeben. Einen weiter gehenden 
Vergleich der Varianten

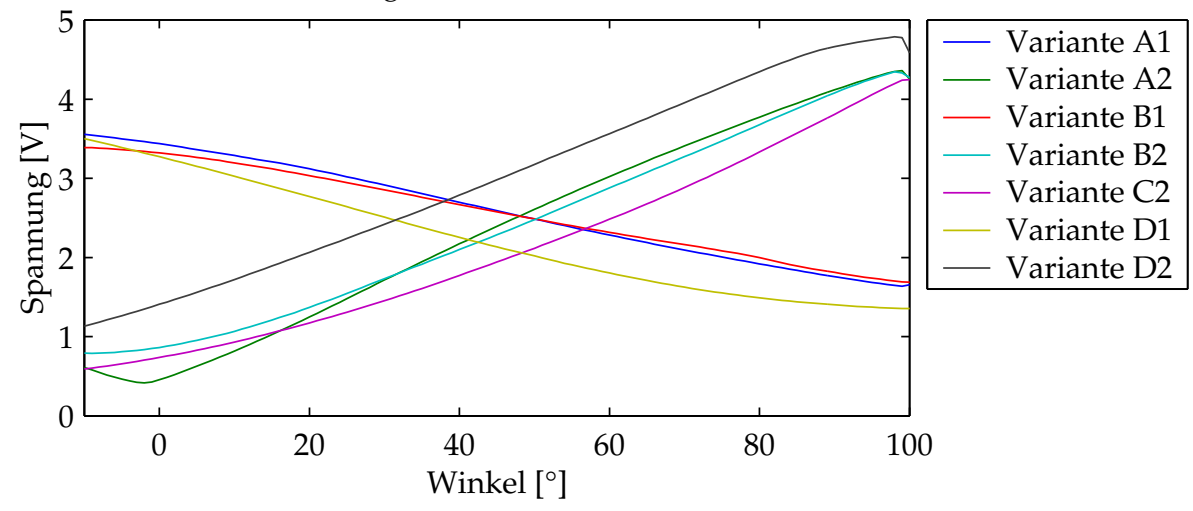

Abbildung 4.17: Aus zehn Messungen gemittelte Signalverläufe der Prototypen beim Beugen der Gelenke von $-10^{\circ}$ bis $+100^{\circ}$.

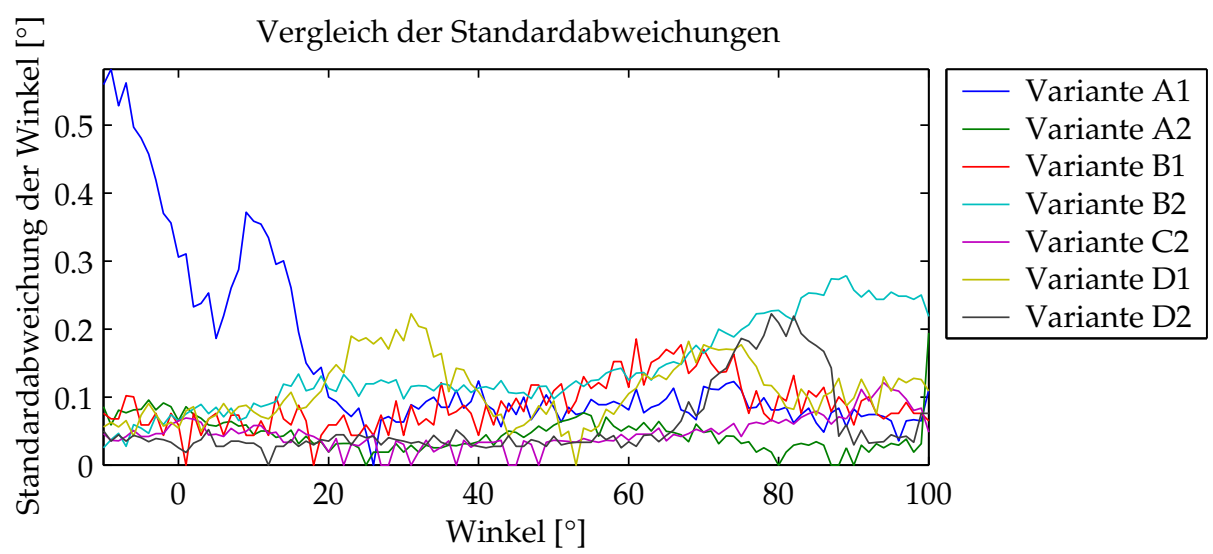

Abbildung 4.18: Standardabweichung der aus den Sensorsignalen von zehn Messungen ermittelten Winkelwerte beim Beugen der Gelenke von $-10^{\circ}$ bis $+100^{\circ}$. 
Blick auf taktile Sensoren und Auswerteverfahren in der Robotik bietet [139], neuere Entwicklungen sind in [108,109] zusammengefasst.

In den folgenden Abschnitten werden verschiedene Ansätze für taktile Sensoren vorgestellt. Sie sind nach Kraftsensoren, Mehrfeldsensoren und Sensorarrays unterschieden. Unter den Kraftsensoren werden diejenigen Sensoren zusammengefasst, die nur einen Messwert je Fingerglied liefern. Mehrfeldsensoren erlauben einen gewissen Rückschluss auf die Kontaktposition oder die Richtung der wirkenden Kraft. Sensorarrays sind regelmäßige Anordnungen kleiner Messaufnehmer, mit deren Hilfe die Form und Lage von Kontaktflächen ermittelt werden kann.

\subsubsection{Kraft- und Drucksensoren}

Unter den Kraft- und Drucksensoren werden alle Prinzipien zusammengefasst, die je Fingerglied ein Summensignal liefern. Diese Sensoren erlauben also einen Rückschluss auf den Betrag der wirkenden Kraft. Weitergehende Details wie Wirkrichtung und Kraftverteilung werden nicht ermittelt.

\section{Drucksensoren}

Sensoren zur Messung von Fluiddrücken können in Verbindung mit einer angepassten Fingerspitze [68] auch zur Messung von Kontaktkräften herangezogen werden. Die Fingerspitze ist aus elastischem Gummi ausgeführt. Sie ist unter der Hautoberfläche mit einer ölgefüllten Fluidkammer ausgestattet (Abbildung 4.19). Bei Objektkontakt erhöht sich der von einem Drucksensor im Inneren der Fingerstruktur aufgenommene Druck in der Fingerspitze.

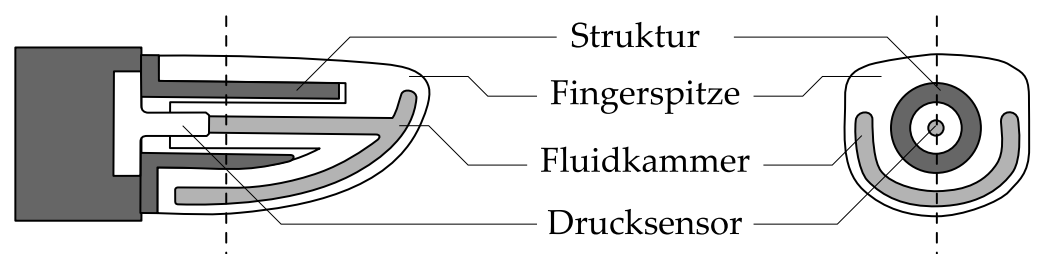

Abbildung 4.19: Taktiler Drucksensor nach [68]. Links: Längsschnitt, Rechts: Querschnitt.

\section{Leitfähige Elastomere}

Leitfähige Elastomere wie z.B. mit Graphit versetztes Silikon ergeben zusammen mit einer isolierenden Abstandshalterschicht und einer Leiterplatte einen Aufbau zur Messung des auf eine Fläche wirkenden Drucks [63].

Im unbelasteten Fall besteht keine elektrische Verbindung zwischen dem leitfähigen Silikongummi und der Leiterplatte (Abbildung 4.20). Mit steigendem Druck wird die Kontaktfläche zwischen den beiden Bauteilen vergrößert, gemessen wird 


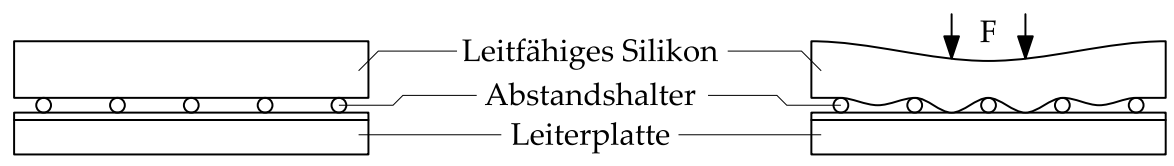

Abbildung 4.20: Taktiler Sensor aus leitfähigem Silikon [63].

ein sinkender Widerstand. Der größte Nachteil dieses Messverfahrens ist das Hystereseverhalten und die geringe mechanische Belastbarkeit des leitfähigen Silikons. Zudem nimmt die Empfindlichkeit des Systems mit steigender Schichtdicke ab.

\section{Kraftabhängige Widerstände}

Eine weit verbreitete Ausführung kraftabhängiger Widerstände sind FSR- (force sensing resistor) Sensoren [78]. Sie sind aus drei beschichteten Folienlagen aufgebaute piezoresistive Sensoren (Abbildung 4.21) mit einer Gesamtdicke von etwa $0,3 \mathrm{~mm}$. Die obere Folienlage ist mit zwei leitfähigen, kammartig ineinander greifenden Strukturen ausgestattet. Sie ist im Randbereich des Sensors durch einen Abstandshalter von der unteren Lage mit einer piezoresistiven Oberfläche getrennt. Beim Einwirken einer Kraft wird zunächst ein Kontakt zwischen den beiden äuBeren Lagen hergestellt, mit steigender Kraft sinkt der Widerstand von mehreren Megaohm um etwa drei Zehnerpotenzen.

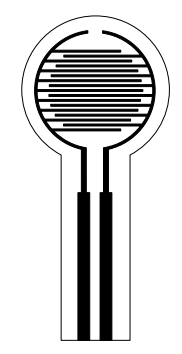

Leitfähige Schicht (1)

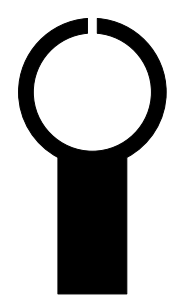

Abstandshalter (2)

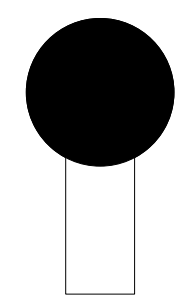

Widerstandsschicht (3)

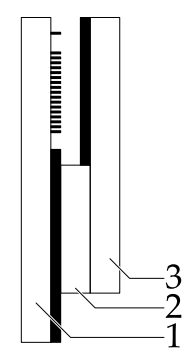

Lagenaufbau (Schnitt, Dicke überhöht)

Abbildung 4.21: Schichtenaufbau von FSR-Sensoren [78].

Neben technischen Anwendungen wie der Sitzbelegungserkennung in Kraftfahrzeugen werden FSR-Sensoren oft in der Physiologie und Ergonomie eingesetzt. Aufgabenstellungen sind die Messung von Greifkräften [49, 69, 82, 127, 179], die teilweise Wiederherstellung des Tastsinns bei Nervenschädigungen [29, 121], die ergonomische Gestaltung von Werkzeuggriffen [201] und die Bestimmung von Fußaufstandskräften [122]. 


\section{Beschleunigungssensoren}

Zur Erkennung des beginnenden Objektkontakts lassen sich in elastischen Fingerspitzen nahe an der Oberfläche angebrachte Beschleunigungssensoren verwenden [75, 76]. Der Aufbau in Abbildung 4.22 zeigt den als Referenz verwendeten Beschleunigungssensor und die in die Silikonhaut integrierten piezoelektrischen Sensoren aus PVDF (Polyvinylidenfluorid, $\mathrm{PVF}_{2}$ ). Der darunter liegende nachgiebige Schaumgummikern erlaubt die Messung von Beschleunigungen bei bereits sehr geringen Kontaktkräften.

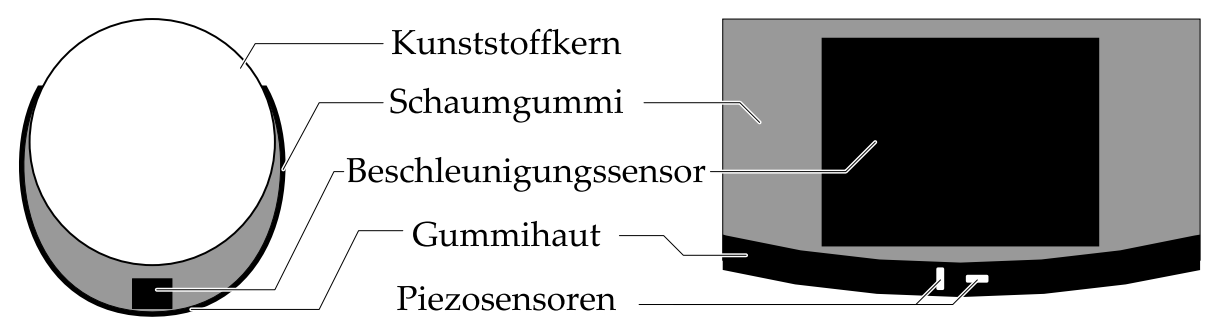

Abbildung 4.22: Testaufbau für taktile Beschleunigungssensoren nach [75]. Links: Schnittdarstellung, Rechts: vergrößerter Ausschnitt mit piezoelektrischen Sensoren in der Gummihaut.

Dieser Aufbau kann auch zur Erkennung eines verrutschenden Objekts verwendet werden. Großen Einfluss auf das Messverhalten hat dabei die Struktur der Hautoberfläche: Eine glatte Silikonoberfläche weist einen sehr ausgeprägten StickSlip-Effekt auf und liefert damit nur einzelne große Signalspitzen. Dieser Effekt wird durch das Anrauen der Oberfläche deutlich reduziert, wobei parallele Riefen die besten Ergebnisse liefern.

Soll das beginnende Verrutschen besonders frühzeitig erkannt werden, so bietet sich die Ausstattung der Oberfläche mit abgerundeten Noppen an [186]. Die Sensoren detektieren hier bei Veränderung der Objektlage kurzzeitig auftretende Schwingungen einzelner Noppen am Rande der Kontaktfläche, ohne dass die gesamte Fingerspitze mit vielen Beschleunigungssensoren auszustatten ist.

\subsubsection{Mehrfeldsensoren}

Mehrfeldsensoren erlauben einen detaillierteren Rückschluss auf die auf ein Fingerglied wirkenden Kräfte. Zusätzliche Parameter sind z.B. die Position des Angriffspunktes, die Wirkrichtung der Kraft oder die Größe der Kontaktfläche. Die Messprinzipien überdecken sich teilweise mit dem vorangegangenen Abschnitt, sind jedoch entweder mit mehreren Sensorfeldern oder als Kombination mehrerer Messprinzipien ausgeführt. 


\title{
Hallsensoren
}

In Kombination mit einer elastischen Silikonschicht und Permanentmagneten lassen sich auch Hallsensoren zur Kräftemessung einsetzen. Aus der wirkenden Kraft resultiert eine Abstandsänderung zwischen Magnet und Hallsensor und damit auch eine Änderung der magnetischen Flussdichte.

Durch die Kopplung von zwei Sensoren (Abbildung 4.23) kann aus Summe und Differenz der Signale auf Betrag und Position einer normal angreifenden Kraft geschlossen werden [103].

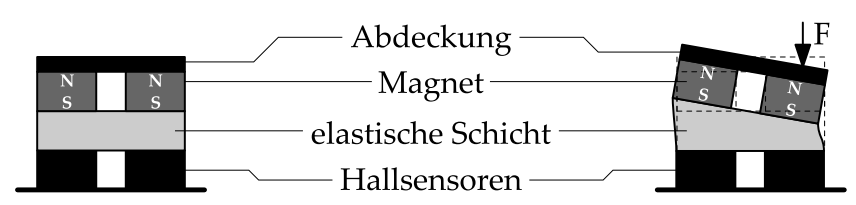

Abbildung 4.23: Schematischer Aufbau eines taktilen Sensors mit Hallsensoren nach [103].

\section{Kraft- und positionsabhängige Widerstände}

Angepasst an den Aufbau der ursprünglich ohne taktile Sensoren entwickelte TUM-Hand [128] wird in [83, 84, 85] eine Kombination aus kraft- und positionsabhängigen Widerständen (FPSR, force and position sensitive resistor) und piezoelektrischen Sensoren beschrieben.
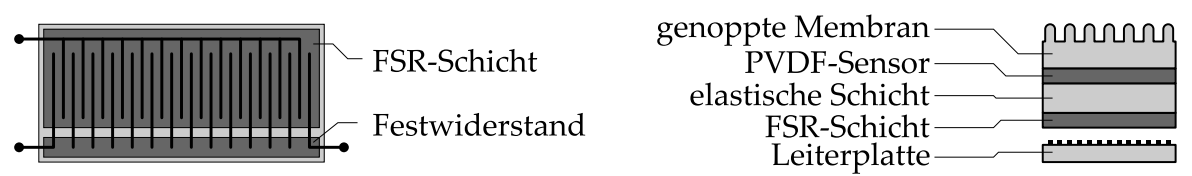

\begin{abstract}
Abbildung 4.24: Links: Schematischer Aufbau eines linearen FPSR-Sensors. Rechts: Schichtenaufbau in Kombination mit PVDFSensor [83].
\end{abstract}

Ein FPSR liefert ähnlich dem bereits beschriebenen FSR ein Kraftsignal. Entlang der Richtung der Positionsbestimmung wirkt ein Festwiderstand in der Kammstruktur als Spannungsteiler (Abbildung 4.24 links). Zur Erfassung von durch Kontakt hervorgerufenen Beschleunigungen befindet sich über dem FPSRSensor zwischen zwei elastischen Schichten ein PVDF-Sensor (Abbildung 4.24 rechts).

In den Fingerspitzen der DLR-Hand I [23, 114] wird ein runder FPSR (Abbildung 4.25 links) mit einem entlang des Umfang liegenden Festwiderstand eingesetzt. Der Korpus der Fingerspitze wird von drei XYZ-FPSR umspannt (Abbildung 4.25 rechts), die aus je zwei linearen FPSR aufgebaut sind. Mit ihnen kann 

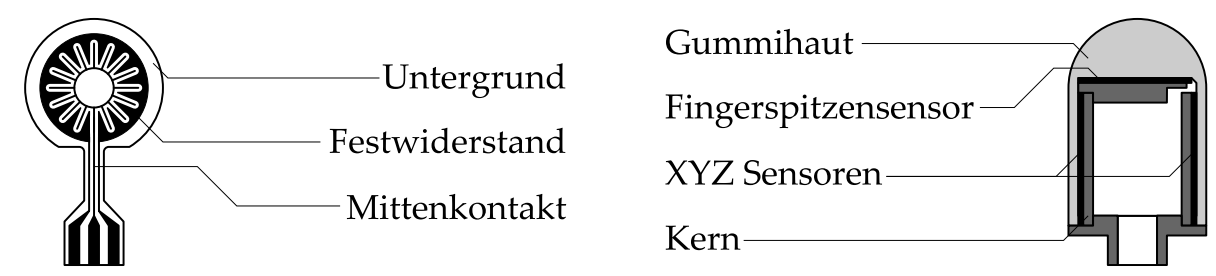

Abbildung 4.25: Links: Runder FPSR-Sensor für Fingerspitzen. Rechts: Anordnung in der Fingerspitze der DLR-Hand I.

die Position des Kraftzentrums sowie der Betrag der Kraft bestimmt werden. Beide Aufbauten weisen an den Übergängen zwischen den einzelnen Messfeldern Bereiche mit einer geringen Empfindlichkeit auf.

\section{Berührungsempfindliche Bildschirme}

Die Kontaktposition von Fingern oder Stiften auf berührungsempfindlichen Bildschirmen wird meist mit resistiven Sensoren bestimmt. Auf der Displayoberfläche sind eine Glas- und eine Folienschicht mit einer leitfähigen Beschichtung aus Indium-Zinn-Oxid (ITO, indium tin oxide) angebracht. Einer der beiden Leiter ist an ihrer rechten und linken Kante, der andere an der oberen und unteren Kante mit Elektroden versehen (Abbildung 4.26 links). Aus dem Spannungsteilerverhalten bei unterschiedlicher Beschaltung kann auf die Lage des Kontaktpunkts geschlossen werden. Kraftinformationen, Kontaktflächen oder mehrere Kontaktpunkte können mit diesem Verfahren nicht ermittelt werden.
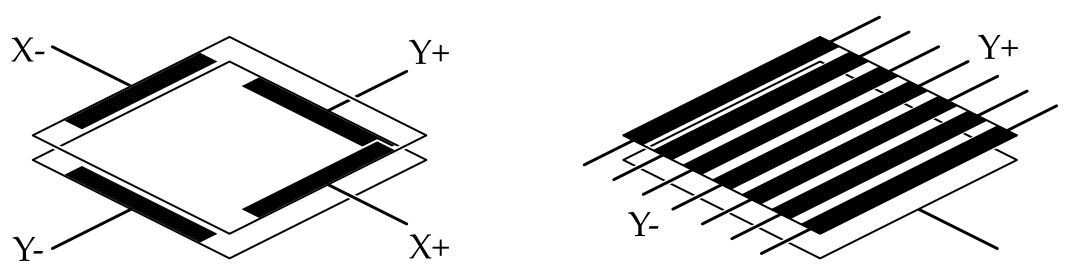

Abbildung 4.26: Links: Schematischer Aufbau eines berührungsempfindlichen Bildschirms. Rechts: resistive Kontaktflächenmessung nach [180].

Ein über das Prinzip des Touchscreens hinaus gehendes Verfahren der resistiven Kontaktflächenmessung ist in [180] beschrieben: Statt der resistiven Folien wird eine Reihe parallel ausgerichteter leitfähiger Gummistreifen und eine leitfähige Unterlage verwendet (Abbildung 4.26 rechts). An jedem Streifen wird mit zwei Messungen der Widerstand zwischen der Unterlage und den beiden Enden bestimmt. Aus diesen Daten wird auf die konvexe Außenkontur des Kontaktbereichs geschlossen. Konkave Konturen können mit diesem Verfahren nicht erfasst werden. 


\subsubsection{Arraysensoren}

Sensorarrays sind regelmäßige Anordnungen vieler kleiner Sensorelemente (Taxels oder auch Tactels, Kurzform von tactile elements). Sie erlauben den Rückschluss auf Form und Kraftverteilung der Kontaktfläche mit dem gegriffenen Objekt.

Die meisten dieser Sensoren sind in einer Matrix aus Zeilen und Spalten angeordnet, die sequentiell zeilen- oder zellenweise ausgelesen wird. Für den Einsatz in Fingern werden Sensorarrays meist mit einer lokalen Elektronik ausgestattet, die die Ansteuerung des Arrays, die Aufnahme der Messwerte und die Datenübertragung auf einem Bus übernimmt. So wird die Auslesegeschwindigkeit der Sensoren nicht durch lange Leitungen und die Zuverlässigkeit des Systems nicht durch eine große Anzahl an Leitungen begrenzt.

Die Leistungsfähigkeit von Sensorarrays ist sehr stark von den verwendeten Auswertungsverfahren abhängig. Aus den Einzelsignalen müssen Merkmale wie z.B. Kontaktkonturen und Mittelpunkte von Kontaktflächen erst noch extrahiert werden. Hierfür werden meist an die Bildverarbeitung angelehnte Algorithmen verwendet.

Eine besondere Herausforderung beim Einsatz von Sensorarrays mit einer elastischen Oberfläche ist die taktile Inversion. Sie beschreibt den Umstand, dass die Signale hochauflösender Sensorarrays durch eine elastische Fingeroberfläche verfälscht werden und nur auf einen Teil der Objektinformationen zurückgeschlossen werden kann [175, 176]. So wirkt sich ein punktförmiger Kontakt an der Hautoberfläche durch das elastische Gewebe hindurch auf einen wesentlich größeren Bereich des darunter liegenden Sensorarrays aus; ein Rückschluss auf Objektgeometrie und Kontaktpunkte an der Fingeroberfläche ist nur unter Annahme von Randbedingungen möglich.

\section{Resistive Arrays}

Bei den Arrays sind resistive Sensoren ein weit verbreitetes Messverfahren. In [63] werden mehrere Entwicklungen der 80er Jahre beschrieben.

Ein Ansatz zu resistiven Arrays ist die Durchsetzung druckempfindlichen Gummis mit einer leitfähigen Gitterstruktur (Abbildung 4.27). Die Literatur beschreibt sowohl die Anwendung in den Fingerspitzen einer Roboterhand [177] als auch die Integration unterschiedlich großer flexibler Teilarrays auf der Handinnenfläche eines Datenhandschuhs [163].

In [158] wird eine Anordnung aus feststehenden vergoldeten Zeilenelektroden und Spaltenelektroden aus leitfähigem Gummi an der Innenseite einer Neoprenhaut als Sensor verwendet. Der Zwischenraum ist mit destilliertem Wasser gefüllt, gemessen wird der Strom, der umgekehrt proportional zum Abstand der beiden Elektroden ist.

Um das Messergebnis eines einzelnen resistiven Sensors nicht durch die parallel geschalteten anderen Sensoren zu verfälschen, müssen die nicht genutzten Zeilen- und Spaltenelektroden des Arrays in einen definierten Zustand gebracht werden. Dies ist entweder Masse (Nullpotential-Methode) oder das vom derzeit 


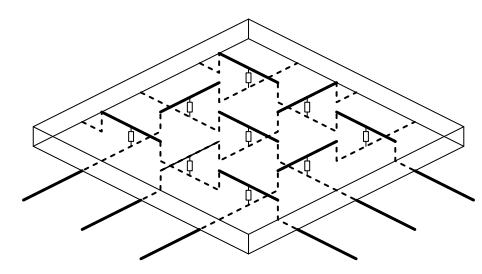

Abbildung 4.27: Array aus druckempfindlichem Gummi mit vernähten Leitern [163, 177].

aktiven Sensor zurückgeführte Potential (Gleichpotential-Methode).

\section{Kapazitive Arrays}

Für die Stanford/JPL-Hand wurde eine Fingerspitze mit einem kapazitiven taktilen Array entwickelt [47]. Es besteht aus flachen Elektroden, die einander überkreuzend in Zeilen und Spalten angeordnet und durch eine elastische Gummischicht als Dielektrikum getrennt sind. Kontaktkräfte verringern den Abstand zwischen den Kondensatorplatten und erhöhen damit seine Kapazität. Das Array wird durch eine externe Elektronik über eine Leitungslänge von etwa $30 \mathrm{~cm}$ ausgelesen. Zur Verringerung von Störungen werden während des Auslesens alle nicht verwendeten Zeilen und Spalten auf Masse geschaltet.
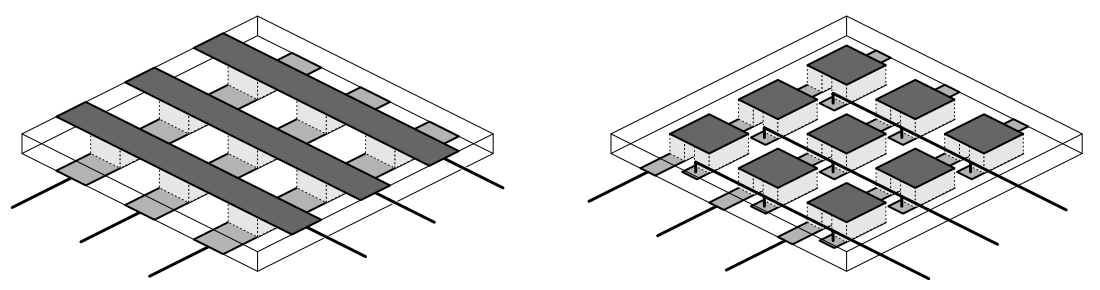

Abbildung 4.28: Schematische Darstellung kapazitiver Sensoarrays: links nach [47], rechts nach [86].

Eine verbesserte Variante dieses Messprinzips wird in der Utah/MIT-Hand eingesetzt [86]. Die bisher verwendeten in Gummi eingebetteten Streifenelektroden werden wegen ihrer Bruchgefahr durch einzelne Platten ersetzt und statt einer werden nun zwei nur noch halb so große feststehende Gegenelektroden verwendet, von denen jeweils eine als Zeilen- und Spaltenelektrode dient. Die Nachteile der hieraus resultierenden kleineren Sensorkapazität werden durch eine in die einzelnen Fingerglieder integrierte Auswerteelektronik kompensiert.

\section{Optische Arrays}

Optische Arrays setzen die von außen wirkenden Kräfte so in optische Signale um, dass sie mit CCD- oder CMOS-Bildwandlern in Videosignale umgesetzt und von Bildverarbeitungssystemen weiter verarbeitet werden können. 
Ein Ansatz hierzu ist die Verwendung eines flachen Lichtleiterkörpers, in den von der Seite Licht so eingekoppelt wird, dass es zwischen den Hauptflächen reflektiert wird [63, 98]. Auf der messenden Seite befindet sich eine lichtundurchlässige, strukturierte elastische Schicht, deren Kontaktfläche zum Lichtleiter sich durch von außen wirkende Kräfte vergrößert (Abbildung 4.29 links). Durch die höhere optische Dichte wird das Licht nun aus dem Lichtleiter ausgekoppelt und durch die berührende elastische Fläche reflektiert. Dieses Lichtsignal wird von einem Bildwandler auf der gegenüberliegenden Seite des Sensors aufgenommen.
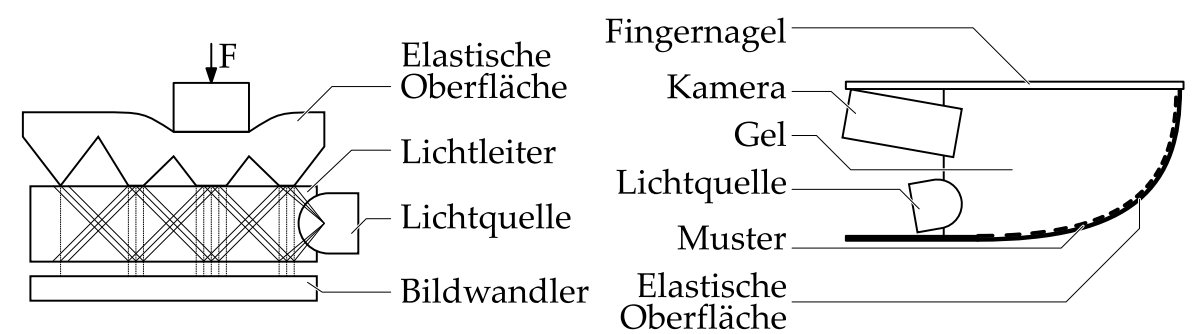

Abbildung 4.29: Schematische Darstellung optischer Arrays. Links: Planares Array mit Lichtleiter. Rechts: Fingerkuppe mit transparenter Gelfüllung.

Alternativ dazu kann mit einer Kamera ein bekanntes Muster auf der Hautinnenseite einer mit einem transparenten Gel gefüllten Fingerkuppe aufgenommen werden (Abbildung 4.29 rechts). Unter Verwendung von Bilderkennungsalgorithmen kann aus der Veränderung des Musters auf die Kraftverteilung an der Hautoberfläche geschlossen werden [77]. Unter Verwendung von Lichtleitern kann dieses Prinzip auch auf größere Hautflächen übertragen werden [200].

\section{Arrays aus Dehnmessstreifen}

Ein auf eine flexible Polyimidfolie aufgebrachtes Array von Dehnmessstreifen wird in [152] genutzt, um die Biegebelastung und damit auch die lokale Geometrie der Haut zu ermitteln. Aus dieser kann wiederum auf die Objektgeometrie im Kontaktbereich geschlossen werden. Als Alternative zum diskreten Aufbau eines solchen Arrays wird in [46] ein Herstellungsverfahren für in die Polyimidfolie eingelassene Dehnmessstreifen und eine Geometrie für den Überlastschutz vorgestellt.

\subsubsection{Anforderungen und Auswahl}

Neben den bereits im Abschnitt 2.3.4 gestellten Anforderungen an Sensoren in einer künstlichen Hand soll ein Kraftsensor die folgenden Anforderungen erfüllen:

Aufgabe: Der Sensor soll bei Kraft- und Präzisionsgriffen eine Rückmeldung zur Kontaktkraft an der Innenfläche des Fingerglieds liefern und eine Überlastungserkennung erlauben. 
Abdeckung des Sensors: Der Einsatz zusammen mit einem nachgiebigen Fingergewebe ist vorzusehen. Zur Vermeidung von Sensorausfällen sind keine Leitungen durch das Fingergewebe zu führen. Ein kosmetischer Schutzhandschuh darf die Funktion des Sensors nicht zu weit einschränken.

Messbereich und Genauigkeit: Der Sensor soll ohne Initialisierung ein absolutes Kraftsignal liefern. Für die Berührungserkennung soll der Sensor im Kraftbereich bis etwa $2 N$ eine hohe Empfindlichkeit aufweisen und etwa die Hälfte des Signalbereichs ausnutzen. Bei größeren Kräften darf die Empfindlichkeit abnehmen, der Sensor darf jedoch durch Überlastung nicht beschädigt werden.

Fingerintegration: Die Tragstruktur der Finger liegt nahe an der Handfläche. Da ihre mechanische Stabilität nicht verringert werden soll, ist der Sensor so flach wie möglich auszuführen.

Die gestellten Anforderungen lassen sich mit Kraftsensoren realisieren, die nur ein Signal je Fingerglied liefern. Die von Mehrfeldsensoren erfassten zusätzlichen Größen wie Kontaktposition entlang des Fingerglieds sind bei der Manipulation von Objekten hilfreich, für das statische Greifen jedoch von geringer Bedeutung. Gegen den Einsatz von Arrays spricht der aufwändigere Aufbau des Sensors sowie der hohe Aufwand für die Aufnahme und Auswertung der Daten.

Unter den vorgestellten Kraftsensoren sind FSR-Sensoren zu favorisieren, da sie den Kriterien des flachen Aufbaus und der Überlastsicherheit genügen. Die Gestaltung des elastischen Oberflächenmaterials kann im Gegensatz zu Sensoren aus leitfähigen Elastomeren unabhängig von Anforderungen an die elektrischen Eigenschaften vorgenommen werden.

\subsubsection{Kraftmessung mit FSR-Sensoren}

\section{Ansprechverhalten}

Zur Erzeugung eines Signals ist zwischen den beiden Folienschichten ein Kontakt herzustellen. Ein frühes Ansprechen wird durch eine kleine Kontaktfläche in der Mitte der Sensorfläche erreicht (Abbildung 4.30 links). Das Signal bei steigenden Kräften wird durch die Größe der Kontaktfläche und den herrschenden Anpressdruck bestimmt. Eine mit steigender Kraft größer werdende Fläche ist daher als vorteilhaft anzusehen. Dies kann auf elegante Weise mit der Anforderung einer elastischen Sensorabdeckung verbunden werden.

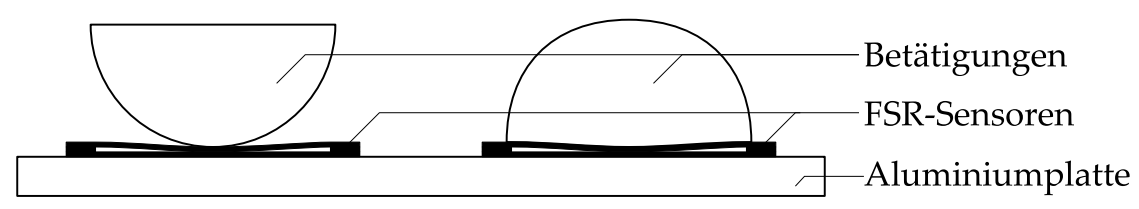

Abbildung 4.30: Betätigung für FSR-Sensoren. 
Die Anbringung der Sensorabdeckung im Randbereich (Abbildung 4.30 rechts) bewirkt die Senkung der Empfindlichkeit und ein späteres Ansprechen, da die Abdeckung schon für die Herstellung des ersten Kontakts verformt werden muss.

FSR-Sensoren sind biegeempfindlich, da beim Beugen auch ohne eine normal zur Oberfläche wirkende Kraft ein Kontakt zwischen Leiterfolie und FSR-Schicht hergestellt wird. Eine der Sensorseiten ist also zur Vermeidung von Messfehlern mit einer ebenen, nicht verformbaren Fläche zu verbinden. Hierfür kann die palmare Seite der Fingerstruktur verwendet werden.

Aus diesen Betrachtungen sind die an verschiedenen Stellen beschriebenen Anordnungen mit einer Abdeckung aus Epoxidharz [69, 82] nicht zu empfehlen. Gleiches gilt für eine rückseitige Polsterung der Sensoren mit Schaum [21].

\section{Vermessung von Sensoren}

Zur Aufnahme von Sensorkennlinien wurde ein Teststand (Abbildung 4.31 links) zur Vermessung und Kalibrierung von Kraftsensoren entwickelt [102]. Die Betätigung des Sensors erfolgt über einen gefederten Stempel, der mit Hilfe eines Gewindebolzens gegen den Sensor gepresst wird. Ein Messrechner zeichnet das Sensorsignal mit einer A/D-Wandlerkarte und die Daten der als Referenz dienenden Laborwaage über eine serielle Schnittstelle auf. Aus dem Messdaten wird die Widerstandskennlininie des Sensors ermittelt (Abbildung 4.31 rechts).
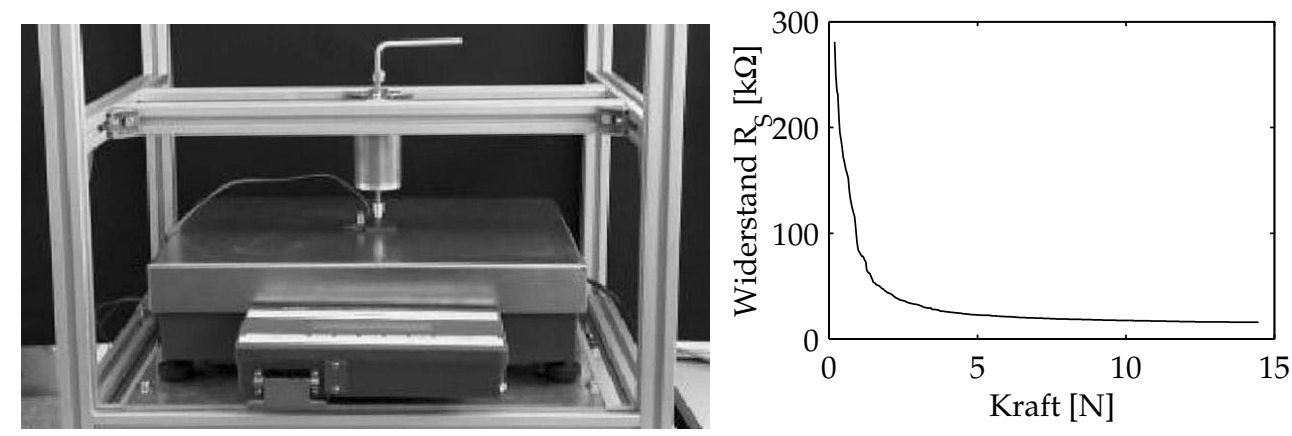

Abbildung 4.31: Links: Teststand zur Vermessung taktiler Sensoren. Rechts: Kraft-Widerstands-Kennlinie eines FSR-Sensors.

\subsubsection{Betätigung und mechanische Integration}

Für den Einsatz im Finger wurde eine neue Sensorabdeckung (Abbildung 4.32) aus Silikon konstruiert, die die Funktionen der Sensorbetätigung mit der Forderung nach einer nachgiebigen Fingeroberfläche verbindet.

Die Abdeckung bildet an beiden Enden die Kontur der Fingerstruktur ab, so dass sie nach der Anpassung der Länge auf sie aufgesetzt werden kann. Im Mittenbereich über dem Sensor befindet sich eine halbkugelförmige Aussparung. Von ihrer Mitte ragt eine halbkugelförmige Sensorbetätigung zur Fingerstruktur hin, 


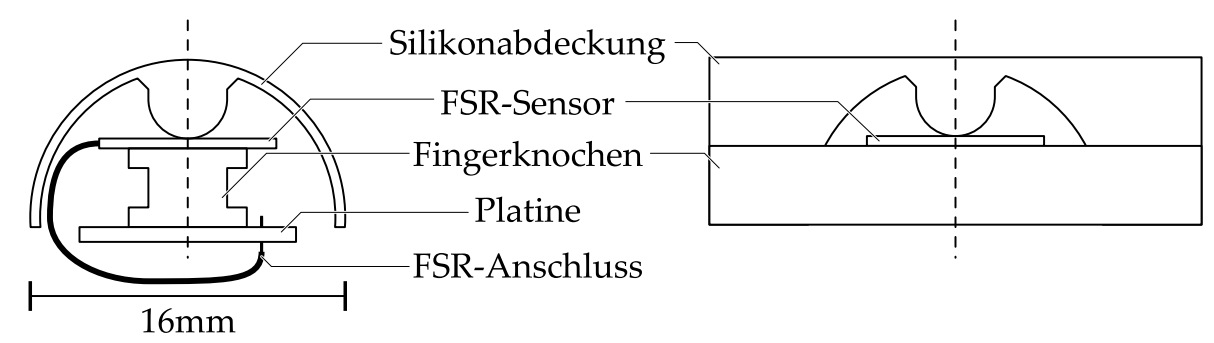

Abbildung 4.32: Quer- und Längsschnitt der FSR-Fingerabdeckung.

sie berührt die Oberfläche des FSR-Sensors (Daten siehe Anhang, Abschnitt 7.1.3) ohne ihn zu betätigen.

Die Musterteile werden in einer hierfür neu angefertigten AluminiumGussform (Abbildung 4.33) aus Zweikomponenten-Silikon hergestellt. Eine weitere Kunststoff-Formhälfte (Abbildung 4.34) ermöglicht die Herstellung von Fingerspitzen mit einer Außenkontur in Form einer Viertelkugel.

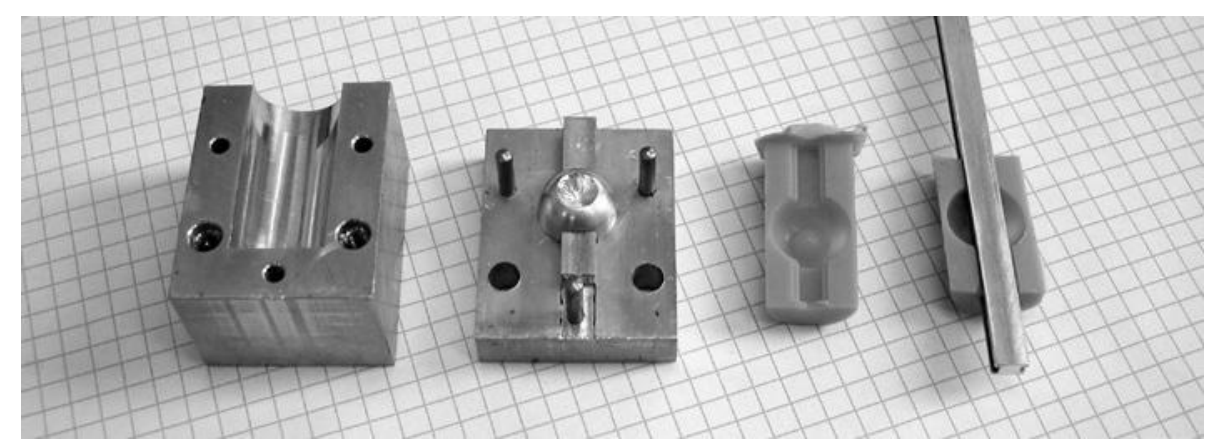

Abbildung 4.33: Form und Musterteile für die Sensorabdeckung der Fingerglieder.

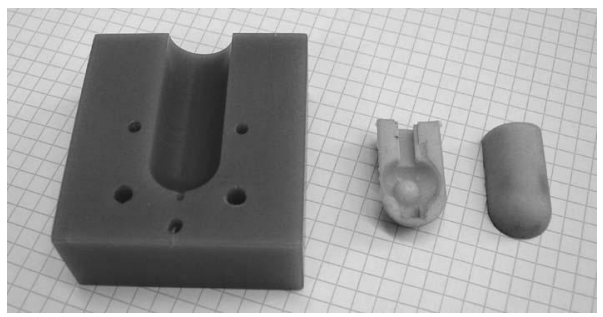

Abbildung 4.34: Formhälfte und Musterteile für die Sensorabdeckung der Fingerspitze. 


\subsubsection{Messverfahren, Sensorkennlinie und Approximation}

\section{Messverfahren}

Die Änderung des Widerstands $R_{\mathrm{S}}$ von FSR-Sensoren kann entweder durch einen Spannungsteiler mit einem Festwiderstand $R_{\mathrm{T}}$ oder einen als Strom-SpannungsWandler beschalteten Operationsverstärker in ein Spannungssignal zur A/DWandlung umgesetzt werden.
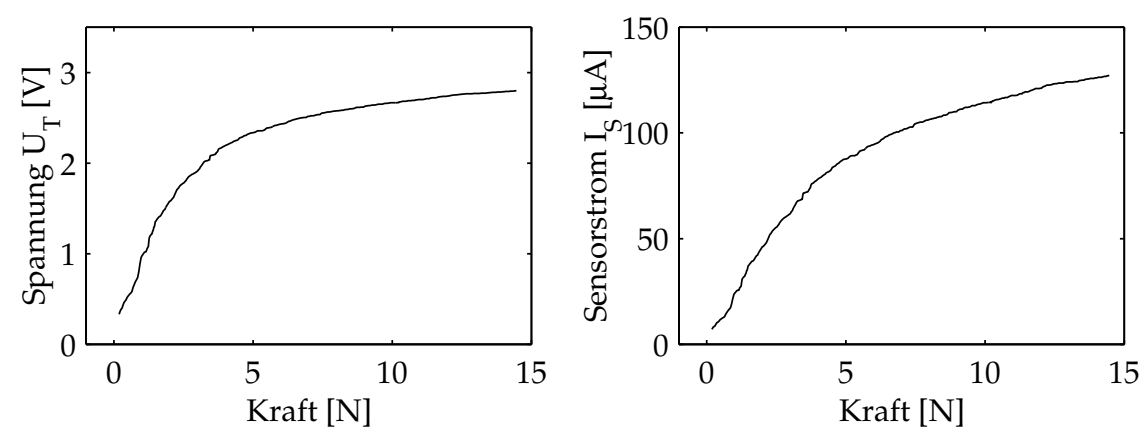

Abbildung 4.35: Kraft-Spannung-Kennlinie bei $U_{\mathrm{V}}=5 \mathrm{~V}$ und $R_{\mathrm{T}}=20 \mathrm{k} \Omega$ (links) und Kraft-Strom-Kennlinie bei $U_{\mathrm{V}}=2 \mathrm{~V}$ (rechts) eines FSR-Sensors (Messung).

Die Wahl des Teilerwiderstands $R_{\mathrm{T}}$ beeinflusst bei der Spannungsteilerschaltung die Nichtlinearität des Sensorsignals. Damit kann auch die Sensorauflösung in den verschiedenen Kraftbereichen gezielt verändert werden (Abbildung 4.35 links). Für den Einsatz in der Hand wurde $R_{\mathrm{T}}=20 \mathrm{k} \Omega$ gewählt, was dem Widerstand des FSR bei einer ausgeübten Kraft von $5 N$ entspricht. Der Widerstand dient gleichzeitig der Begrenzung des Maximalstroms auf einen zulässigen Wert.

Der Signalverlauf bei der Strom-Spannungs-Wandlung flacht im oberen Kraftbereich weniger ab als bei der Spannungsteilerschaltung. Dabei darf zur Einhaltung des maximal zulässigen Sensorstroms die Versorgungsspannung $2 \mathrm{~V}$ nicht überschreiten (Abbildung 4.35 rechts). Die Leistungsaufnahme des Sensors liegt in beiden Fällen deutlich unter dem zulässigen Wert. Für Kräfte bis etwa $3 N$ ist die Leistungsaufnahme der Spannungsteilerschaltung geringer als die des StromSpannungs-Wandlers.

Gewählt wird die Spannungsteilerschaltung, da sie mit der gleichen Versorgungsspannung wie die Hallsensoren von $5 V$ betrieben werden kann. Für die Strom-Spannungs-Wandlung ist dagegen eine separate Versorgung oder ein Spannungsregler in den Fingergliedern notwendig.

\section{Approximation der Sensorkennlinie}

Die direkte Schätzung der wirkenden Kraft aus der gemessenen Spannung durch Polynommodelle lieferte keine befriedigenden Ergebnisse. Gute Ergebnisse wurden dagegen bei der Approximation der doppelt logarithmischen Kraft- 
Widerstands-Kennlinie (Abbildung 4.36 links) erreicht. Die Widerstandswerte wurden dabei nach Gleichung (4.12) aus den Spannungswerten und dem Teilerwiderstand berechnet.

$$
R_{\mathrm{S}}=R_{\mathrm{T}} \cdot\left(\frac{U_{\mathrm{V}}-U_{\mathrm{T}}}{U_{\mathrm{T}}}\right)
$$
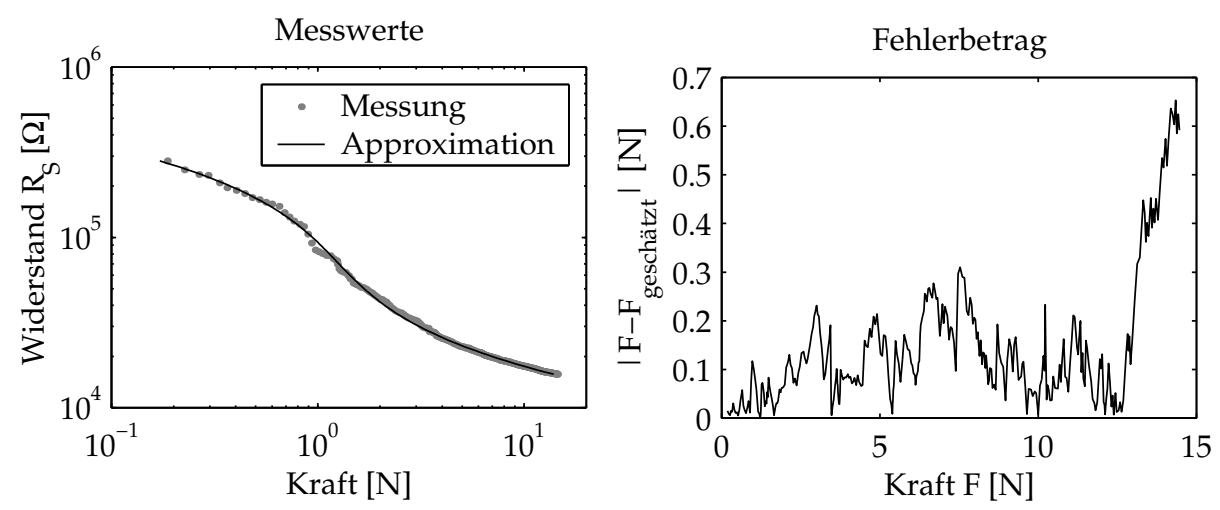

Abbildung 4.36: Links: Aus Spennungswerten berechnete und approximierte doppelt logarithmische Kraft-Widerstands-Kennlinie eines FSR-Sensors. Rechts: Fehler bei Approximation mit Polynom dritter Ordnung.

Nach der Methode der kleinsten Fehlerquadrate wurden für die Widerstandskennlinie die Koeffizienten $d_{i}$ in Gleichung (4.13) ermittelt.

$$
\begin{aligned}
\log \hat{F} & =\sum_{i=0}^{n} d_{i} \cdot\left(\log R_{\mathrm{S}}\right)^{i} \\
& =\sum_{i=0}^{n} d_{i} \cdot\left(\log \left(R_{\mathrm{T}} \cdot \frac{U_{\mathrm{V}}-U_{\mathrm{T}}}{U_{\mathrm{T}}}\right)\right)^{i}
\end{aligned}
$$

Aus der Fehlerkennlinie in Abbildung 4.36 rechts ist zudem ersichtlich, dass durch die Logarithmierung der Bereich der kleinen Kräfte sehr gut abgebildet wird ohne dass bei Kräften oberhalb von $10 \mathrm{~N}$ zu große Fehler in Kauf genommen werden müssen. Durch den größeren Rechenaufwand im Vergleich zur direkten Approximation der gemessenen Spannungs-Kraft-Kennlinie wird zusätzlich ein gutes Extrapolationsverhalten über den Kalibrierungsbereich hinaus erreicht.

\section{Sensorauflösung}

Aus der nichtlinearen Kennlinie ergibt sich bei Verwendung eines 10 bit-A/DWandlers die in Abbildung 4.37 gezeigte Kraftauflösung. So wird auch ohne optimale Ausnutzung des Messbereichs für Kräfte unter $2 N$ eine Auflösung besser 
als $\frac{10 \mathrm{mN}}{\text { bit }}$ erreicht, bis $15 \mathrm{~N}$ steigt dieser Wert um den Faktor 20. Zum Vergleich erreicht ein linearer Sensor mit einem Messbereich von $15 N$ bei optimaler Nutzung des Messbereichs eine Auflösung von $\frac{15 \mathrm{mN}}{\text { bit }}$.

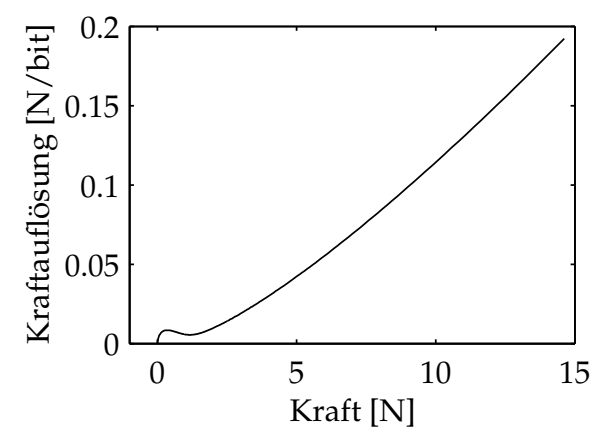

Abbildung 4.37: Kraftauflösung des FSR-Sensors bei 10 bit Wandlerauflösung.

\subsubsection{Messung von Greifkräften}

Eine Abwandlung des für die künstliche Hand entwickelten Kraftsensors wurde für die Messung von Greifkräften der menschlichen Hand und verschiedener Handprothesen entwickelt. Das Ziel war hierbei der Vergleich der Greifkraftverteilung auf den Fingerinnenseiten und der Handfläche [90, 91, 92, 93, 94].

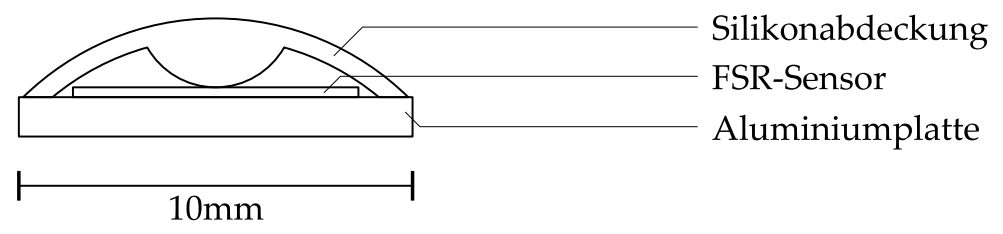

Abbildung 4.38: Schnittdarstellung der zur Messung von Greifkräften verwendeten FSR-Anordnung.

Für die Sensorabdeckung wurde die Halbkugelform der Betätigung und die steife Ausführung der Sensorrückseite übernommen (Abbildung 4.38). Als Träger dient eine quadratische Aluminiumplatte mit $10 \mathrm{~mm}$ Kantenlänge, die an ihrer Rückseite mit einem größenverstellbaren Klettband vernäht ist (Abbildung 4.39). So kann der Sensor an Fingern unterschiedlichen Durchmessers befestigt werden. Bei Messungen im Bereich der Handfläche erfolgt die Positionierung mit doppelseitigem Klebeband.

Das Kalibrierverfahren und die Approximationsmethode der Sensorkennlinie sind von den fingerintegrierten Sensoren übernommen. Die Kontaktkräfte der 20 Messpunkte werden über den Greifzeitraum gemittelt und auf einer schematisch dargestellten Hand visualisiert. 


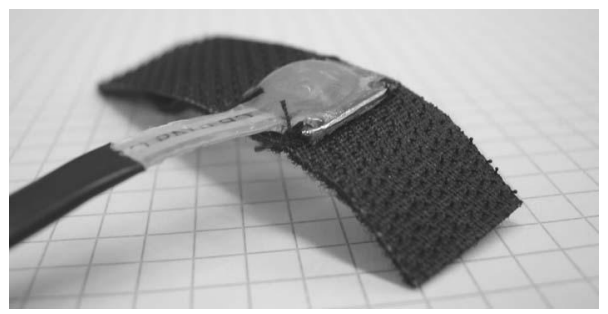

Abbildung 4.39: Sensor zur Greifkraftmessung mit Klettbandbefestigung.
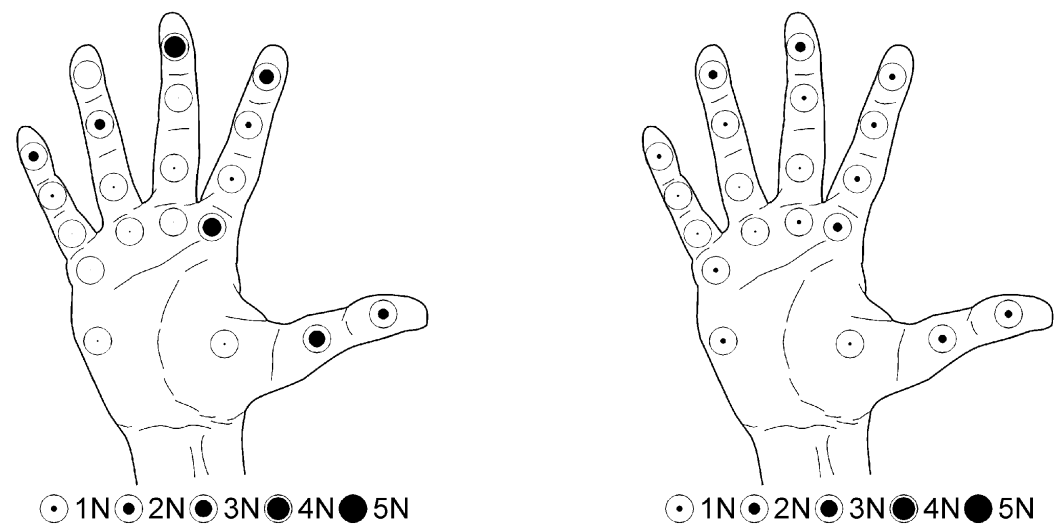

Abbildung 4.40: Vergleich der Kontaktkraftverteilung beim Greifen eines zylindrischen Gegenstands: FZK-Hand (links) und menschliche Hand (rechts) nach [90].

Der Vergleich der Kontaktkraftverteilung zeigt eine große Ähnlichkeit von menschlicher Hand und FZK-Handprothese (Abbildung 4.40). Die Kontaktpunkte verteilen sich über alle Finger und die Handfläche. Konventionelle Handprothesen mit nur einem Freiheitsgrad und starren Fingern heben sich hiervon deutlich ab (Abbildung 4.41). Sie weisen nur wenige Kontaktpunkte mit hohen Kontaktkräften auf, womit auch die Gefahr der Beschädigung eines zu greifenden Objektes höher ist. Für die gestellte Greifaufgabe lässt sich damit zeigen, dass das Greifverhalten der vielgliedrigen Handprothese mit flexiblen Aktoren der menschlichen Hand näher kommt als konventionelle Prothesen.

\subsection{Momentensensoren}

Die Bestimmung von in Fingern wirkenden Momenten und Kräften ist neben der Messung der Gelenkpositionen eine weitere Analogie zur Propriozeption des Menschen. Sie dient bei der Ausführung von Greifvorgängen primär der Begrenzung und Regelung der durch die Finger ausgeübten Momente. So können nicht 

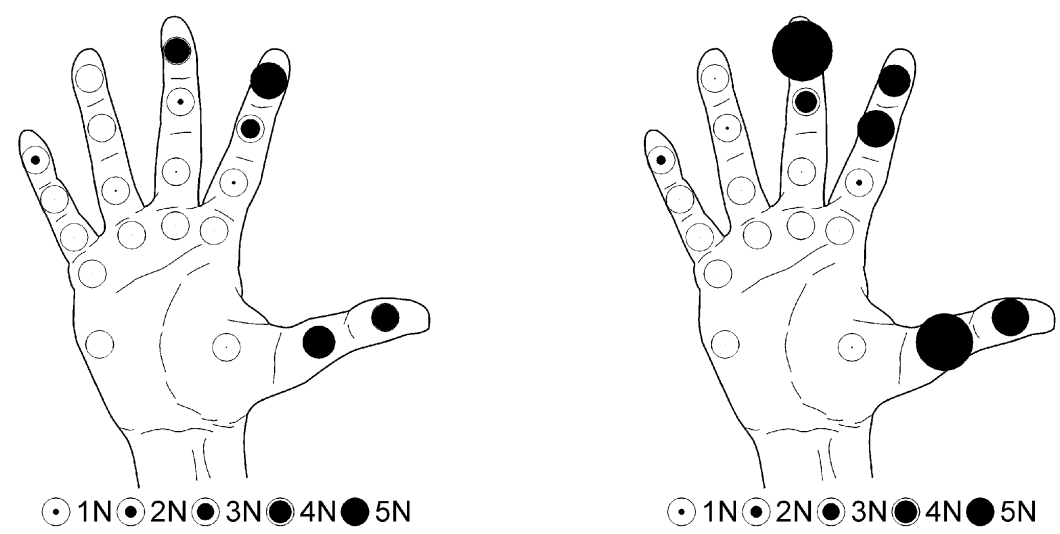

Abbildung 4.41: Vergleich der Kontaktkraftverteilung beim Greifen eines zylindrischen Gegenstands: Otto Bock Systemhand (links) und Otto Bock Sensorhand (rechts) nach [90].

nur zu greifende Objekte, sondern auch der Antrieb und die Struktur der Hand vor Beschädigungen durch Überlastung geschützt werden.

$\mathrm{Zu}$ unterscheiden ist zwischen der Messung an der Struktur der Finger und der Messung an den Antrieben. In der Fingerstruktur sind besonders die Fingerspitzen von hohem Interesse, da sie in die meisten Greifvorgänge einbezogen sind. Die antriebsseitige Messung erfolgt entweder an den Aktoren selbst oder in den Kraftübertragungen zu den Gelenken.

In der Robotik werden multiaxiale Kraft-Momenten-Sensoren oft im Bereich des Handgelenks eingesetzt. Sie liefern aus den Signalen mehrerer Dehnmessstreifen die Kräfte und Momente entlang der drei Hauptachsen. Aus diesen Informationen kann auf Masse und Schwerpunktlage eines gegriffenen Objekts geschlossen werden. Die Optimierung solcher Strukturen wird in [19] ausführlich diskutiert.

\subsubsection{Multiaxiale Kraft-Momenten-Sensoren}

Miniaturisierte Kraft-Momenten-Sensoren werden vor allem in den Fingerspitzen künstlicher Hände eingesetzt. Die Drehmomente und Kräfte werden meist über die Messung der elastischen Materialdehnung an mehreren Stellen der Tragstruktur des Sensors bestimmt. Aus ihrem bekannten Nachgiebigkeitsverhalten können die einzelnen Kraft- und Momentenkomponenten berechnet werden.

Die Annahme, dass nur an einer Stelle ein Objektkontakt herrscht, erlaubt die Lagebestimmung des Kontaktpunkts. Die dabei durch Messfehler auftretenden Abweichungen von der Fingerkontur sind zu kompensieren. Die gewonnenen Informationen sind für die Manipulation von mit den Fingerspitzen gegriffenen $\mathrm{Ob}-$ jekten hilfreich. Die in den Antrieben wirkenden Kräfte können hier über die Fingerkinematik berechnet werden. Als Randbedingung gilt dabei, dass außer an der Fingerspitze keine Kräfte auf den Finger wirken. 


\section{Zylindrischer PSD-Sensor}

Eine optische Messung der in Normalrichtung zur Längsachse wirkenden Kräfte und der Position des Kraftangriffspunkts lässt sich mit der Anordnung in Abbildung 4.42 erreichen [140, 145]. Das Fingerglied ist aus zwei konzentrischen Hohlzylindern aufgebaut, die durch elastische Ringe auf Abstand gehalten werden. An den Enden des äußeren Zylinders sind zwei 2-D PSDs (position sensitive detectors) angebracht. Sie erfassen die Positionsänderungen von zwei durch LEDs am inneren Zylinder erzeugten Lichtpunkten. Aus Betrag und Richtung der Positionsänderungen wird über die Nachgiebigkeit der Gummiringe auf den Betrag und den Angriffspunkt der Kraft geschlossen. Kräfte in Richtung der Längsachse und Momente um die Längsachse sind separat zu bestimmen.

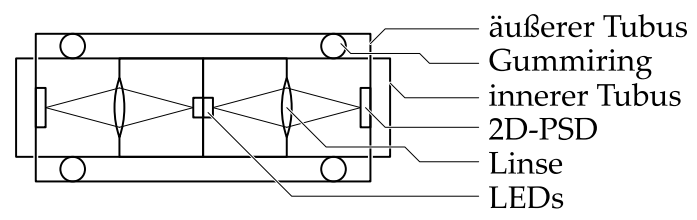

Abbildung 4.42: Optischer Kraft-Momentensensor mit PSD.

\section{DMS-Sensoren der Karlsruher Hand I und II}

Der multiaxiale Kraft-Momenten-Sensor in der Karlsruher Hand I basiert auf einem Kunststoffzylinder mit vier Dehnmessstreifen (Abbildung 4.43 links). Aus den Sensorsignalen lassen sich die Kraft in Längsrichtung des Fingers sowie die beiden quer dazu wirkenden Momente berechnen. Die quer zur Längsachse wirkenden Kräfte und das um die Längsachse wirkende Torsionsmoment werden nicht bestimmt [41,198]. Die halbkugelförmige Fingerspitze ist zusätzlich mit einer Oberfläche aus mehreren Kraftsensoren ausgestattet.
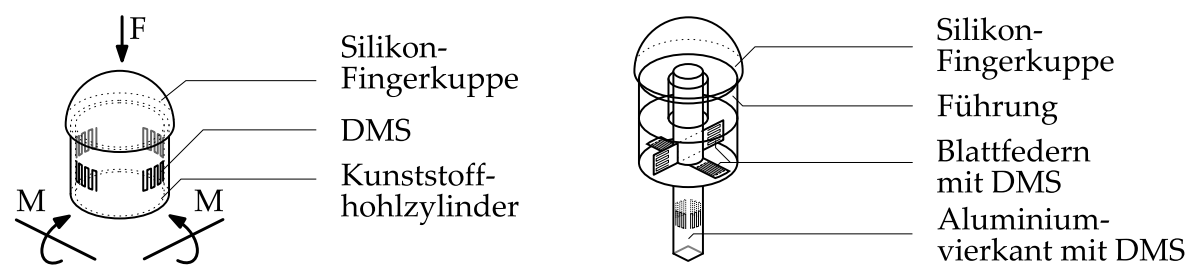

Abbildung 4.43: Multiaxiale Kraft-Momenten-Sensoren der Karlsruher Hand I (links, 3D) und der Hand II (rechts, 6D).

In der neueren Karlsruher Hand II [55] wird ein optimierter Sensor (Abbildung 4.43 rechts) eingesetzt, der mit acht Dehnmessstreifen ausgestattet ist. Die bisher fehlenden Größen werden über die Dehnung zweier Blattfedern bestimmt, die durch die geführte Fingerspitze betätigt werden. 


\section{DLR 6D-Kraft-Momenten-Sensor}

Für die Finger der DLR-Hand II [22] wurde ein miniaturisierter 6D-KraftMomenten-Sensor entwickelt. Die Tragstruktur mit zehn Dehnmessstreifen (Abbildung (4.44) und Auswerteelektronik sind dabei in ein zylindrisches Gehäuse (Durchmesser $20 \mathrm{~mm}$, Länge $16 \mathrm{~mm}$ ) integriert.

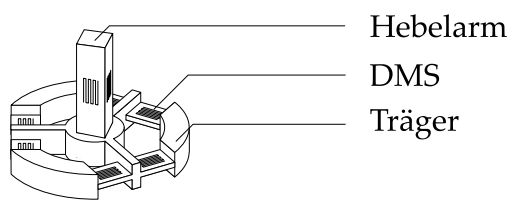

Abbildung 4.44: Tragstruktur des 6D-Kraft-Momenten-Sensors der DLRHand II.

\subsubsection{Gelenkmomentsensoren}

Das an das Gelenk abgegebene Antriebsmoment kann in vielen Fällen aus am Aktor oder im Antriebsstrang gemessenen Größen bestimmt werden, teilweise unter Zuhilfenahme weiterer Messwerte. Im statischen Fall beinhaltet das Übertragungsmodell nur die Übersetzungsverhältnisse zwischen gemessener Größe und Antriebsmoment. Bei dynamischen Vorgängen sind zusätzlich Reibung und Trägheitsmoment des Systems zu berücksichtigen. Die Belastung der einzelnen Antriebe ist hier einfacher zu bestimmen als mit Fingerspitzensensoren.

\section{Dehnmessstreifen}

Die Erfassung der Momente erfolgt in allen Gelenken der DLR-Hand I [23] mit Dehnmessstreifen, die unmittelbar distal der Gelenke an der Fingerstruktur angebracht sind. Sie erlauben die Messung von Gelenkmomenten bis zu 1,8 $\mathrm{Nm}$ bei einer Auflösung von 9 bit. Messbereich und Auflösung wurden in der zweiten Generation auf maximal 4,8 $\mathrm{Nm}$ und 11 bit angehoben.

\section{Druckmessung}

Bei pneumatisch und hydraulisch angetriebenen Fingergelenken kann aus einer Druckmessung zwischen Ventil und Aktor auf das Gelenkmoment geschlossen werden.

Ein Beispiel ist die TUM-Hand [128], bei der sich jedoch das Übertragungsverhalten der hydraulischen Strecke negativ auf die Qualität der Messungen auswirkt [194, 195]. Quellen für Messfehler sind die relativ ungenaue, ebenfalls extern liegende Positionssensorik, Stick-Slip-Effekte in den Zylindern sowie das Übertragungs- und Ausdehnungsverhalten des etwa $4 \mathrm{~m}$ langen Verbindungsschlauchs. 


\section{Zugkraftmessung an Seilzügen}

In seilzuggetriebenen Händen wie der Stanford/JPL-Hand [162] wird das ausgeübte Gelenkmoment durch Messung der Seilzugkraft ermittelt. Da die direkte Messung mit in den Seilzügen angebrachten Zugkraftsensoren oft zu einem Bruch der Sensorleitungen führt, wird ein indirektes Messverfahren verwendet. Eingesetzt werden dazu Dehnmessstreifen an einem Biegebalken mit Umlenkrolle (Abbildung 4.45).

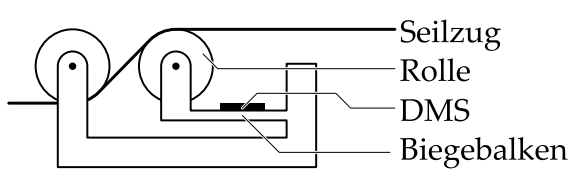
Abbildung 4.45: Schema der Zugkraftmessung am Seilzug in der
Stanford/JPL-Hand [162].

\section{Strommessung}

Bei motorisch angetriebenen Gelenken ist unter bestimmten Voraussetzungen eine Momentenmessung über die Stromaufnahme des Motors möglich [41]. Die Strommessung wird mit Hilfe eines niederohmigen Reihenwiderstands oder über die Messung der magnetischen Flussdichte in direkter Nähe des Leiters durchgeführt. Da selbsthemmende Getriebe keine Momente vom Fingerglied zum Motor zurück übertragen, ist dieses Verfahren nicht zur Überwachung der Antriebsbelastung im Stillstand geeignet.

\subsubsection{Anforderungen und Auswahl}

Die Auswahl der Momentensensoren ist aufgabenspezifisch zu betrachten: Die Momentenmessung in den Gelenken ist in allen Fällen für die Überwachung des Antriebs wichtig, um drohende Aktorschäden durch Überdruck zu vermeiden. Sie erlaubt bei Kraftgriffen mit mehreren Objektkontaktpunkten eine Abschätzung der ausgeübten Kontaktkräfte. Soll eine Hand darüber hinaus für die Manipulation von Objekten eingesetzt werden, ist der Messung in den Fingerspitzen eine hohe Bedeutung zuzumessen, da sie präzise Kontaktinformationen für die Regelung der Gelenke liefert.

Für eine von flexiblen Fluidaktoren angetriebene Hand, die vorrangig zum statischen Greifen von Objekten eingesetzt werden soll, ergeben sich zusätzlich zu den allgemeinen Anforderungen aus Abschnitt 2.3.4 somit folgende Einzelanforderungen:

Aufgabe: Zu bestimmen ist das vom Aktor ausgeübte Drehmoment und der Aktordruck. 
Räumliche Anordnung: Die Sensoren sind außerhalb der Finger zwischen Ventil und Aktor anzubringen. Zur Vermeidung von Messfehlern ist die Anbringung in der Handfläche oder im Unterarm zu realisieren, größere Entfernungen sind zu vermeiden.

Medienunabhängigkeit: Die Drucksensoren sollen sowohl im pneumatischen als auch im hydraulischen Betrieb eingesetzt werden können.

Überlastsicherheit: Die Sensoren sind überlastsicher auszulegen. Sie haben also auch über den maximal zulässigen Aktordruck hinaus ein Signal zu liefern und dürfen nicht beschädigt werden.

Die gestellten Aufgaben lassen sich über die direkte Messung des Aktordrucks realisieren. Das Gelenkmoment ist unter Berücksichtigung der Gelenkposition über das winkelabhängige Momentenkennfeld des Aktors zu berechnen.

Im Fall einer von flexiblen Fluidaktoren angetriebenen Hand ist die Messung des Aktordrucks das zu favorisierende Verfahren. Die bei Zylindern nachteiligen Stick-Slip-Effekte durch die Reibung an den Kolbendichtungen treten bei diesen Aktoren nicht auf. Eine Positionsregelung mit einem unterlagerten Druckregelkreis und die Überwachung des Aktordrucks sind direkt möglich.

\subsubsection{Druckmessung in flexiblen Fluidaktoren}

\section{Pneumatische Drucksensoren}

Für Versuche zur pneumatischen Druck- und Positionsregelung [62] wurde ein Sensorprototyp mit einer Absolutdruck-Messzelle der Fa. Keller [97] aufgebaut. Die Silizium-Messzelle $\left(2,8 \times 2,8 \mathrm{~mm}^{2}\right)$ mit einer Wheatstone-Brücke befindet sich ohne weiteren Schutz vor dem Druckmedium auf einem flexiblen Polyimid-Träger (Abbildung 4.46 links; Daten siehe Anhang, Abschnitt 7.1.4).

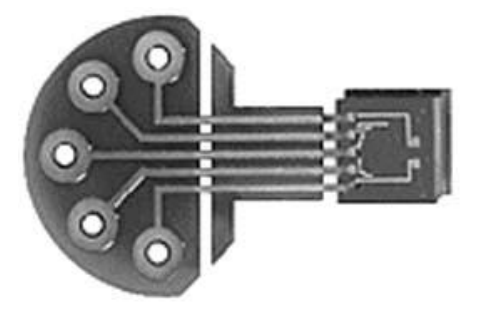

Abbildung 4.46: Druckmesszelle für pneumatische Druck- und Positionsregelung. Die quaderförmige Druckmesszelle befindet sich an der rechten Seite. Der linke Bereich wird zur Kontaktierung verwendet.

Das Spannungssignal wird von einem Differenzverstärker auf einen Spannungsbereich von $0 \ldots 5 \mathrm{~V}$ verstärkt. Als weitere Funktionen sind ein Nullpunktabgleich mit Potentiometer und ein Tiefpassfilter erster Ordnung realisiert. Letzteres filtert die durch die gepulste Ansteuerung der Ventile erzeugten Druckschwingungen aus dem Sensorsignal. 


\section{Hydraulische Drucksensoren}

Im hydraulischen Betrieb muss die Materialkompatibilität zwischen Sensor und verwendetem Medium besonders beachtet werden. Dies betrifft insbesondere die verwendeten Dichtungen, die nicht angegriffen werden dürfen. Für das verwendete Silikonöl sind nur Dichtungen aus Fluor-Silikon-Kautschuk, EthylenPropylen-Dien-Monomer (EPDM) und Neopren einzusetzen. Der verbreitete Silikon-Kautschuk darf nicht gewählt werden, da er durch Silikonöl aufgelöst wird.

Da das System steifer als im Betrieb mit kompressiblen Medien ist, müssen die gewählten Sensoren auch kurzfristige starke Druckanstiege bis zum Eingriff einer Drucküberwachung zulassen. Für Tests wurden Messzellen der Serie 24PC von Honeywell (Abbildung 4.47; Daten siehe Anhang, Abschnitt 7.1.4) gewählt [74], die den Druck relativ zur Umgebung messen.

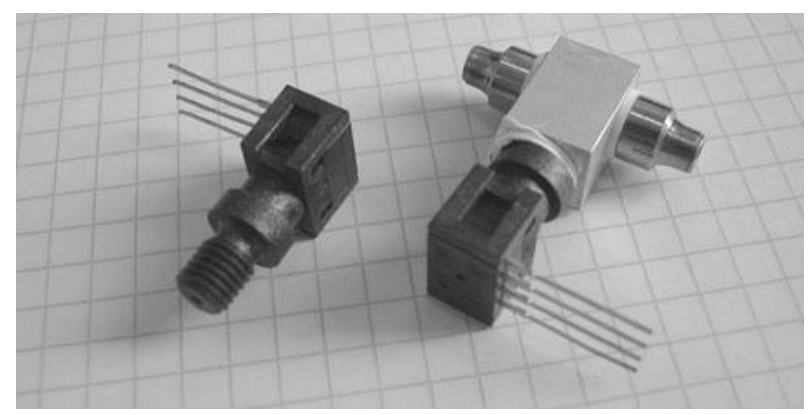

Abbildung 4.47: Drucksensor für hydraulischen Betrieb.

\subsubsection{Dimensionierung}

Die Auslegung eines Drucksensors wird im hauptsächlich durch seinen Einsatz bei der Gelenkregelung und der Momentenschätzung bestimmt.

\section{Sensorauflösung}

Für die Herleitung der erforderlichen Auflösung des Drucksensors wird auf die erst in Abschnitt 5.1 eingeführte Positionsregelung pneumatisch betriebener Gelenke vorgegriffen. Diese wird dort mit einem dem Positionsregelkreis unterlagerten Druckregler realisiert. Dabei wird der statische Zusammenhang zwischen Druck und Gelenkposition über die aus dem Momentenkennfeld des Aktors entnommene Nullmomentenkennlinie (Abbildung 4.48 links) hergestellt.

So muss der Drucksensor mindestens Druckdifferenzen messen können, die über diese Kennlinie dem bereits für den Winkelsensor geforderten maximalen Positionsfehler von $\pm 0,5^{\circ}$ entsprechen. Dies erfolgt über die winkelabhängige Steigung der Nullmomentenkennlinie $\frac{\Delta \varphi}{\Delta p}(\varphi)$ (Abbildung 4.48 rechts).

Der Aktor reagiert im mittleren Winkelbereich mit bis $\mathrm{zu} \frac{90^{\circ}}{10^{5} \mathrm{~Pa}}$ auf Druckänderungen. Entsprechend der Winkelmindestauflösung von $\frac{0,5^{\circ}}{b i t}$ ist daraus eine 

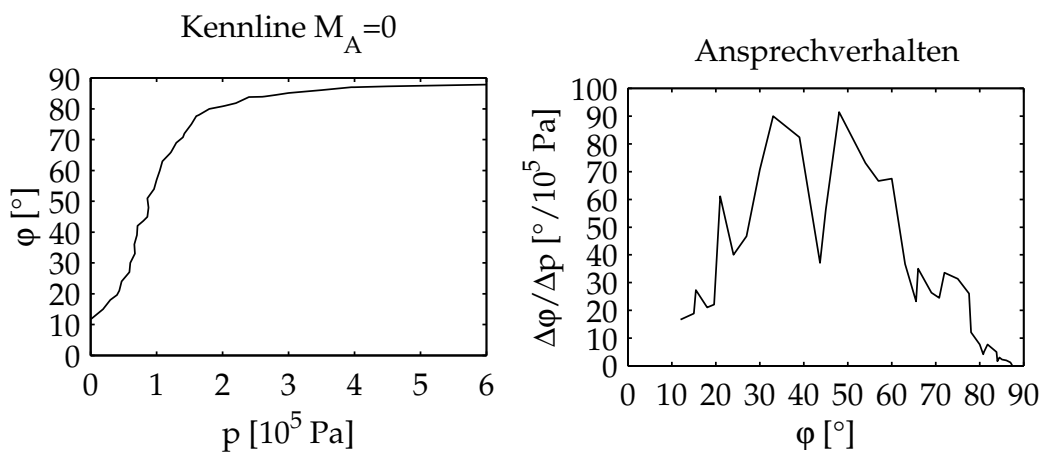

Abbildung 4.48: Links: Nullmomentenkennlinie des Aktors. Rechts: winkelabhängige Empfindlichkeit $\frac{\Delta \varphi}{\Delta p}(\varphi)$ des Aktors auf Druckänderungen (aus Messdaten).

Mindestdruckauflösung von $\frac{10^{5} P a}{90^{\circ}} \cdot \frac{0,5^{\circ}}{b i t}=\frac{5,5 \mathrm{hPa}}{b i t}$ abzuleiten. Mit einem Messbereich von $10 \cdot 10^{5} \mathrm{~Pa}$ ergeben sich mindestens 1800 Schritte, also 11 bit Wandlerauflösung.

\section{Momentenschätzung}

Die Schätzung des Aktormoments aus Winkel und Aktordruck basiert auf dem in Abschnitt 3.2.1 hergeleiteten Momentenkennfeld. Zur Reduktion des Schätzfehlers werden nach Gleichung (4.14) höhere Polynomgrade verwendet. Die insgesamt 12 Parameter wurde mit Hilfe eines Matlab-Programms nach der Methode der kleinsten Fehlerquadrate ermittelt.

$$
M_{\mathrm{A}}=\sum_{i=0}^{2} \sum_{j=0}^{3} a_{i j} \cdot \varphi^{j} \cdot p^{i}
$$

Diese Maßnahme bewirkt eine Reduktion des Approximationsfehlers $\left|\Delta M_{A, \max }\right|$ (Abbildung 4.49) auf 0,02 Nm, womit der maximale relative Momentenfehler auf $\frac{0,02 \mathrm{Nm}}{0,6 \mathrm{Nm}}=3,3 \%$ sinkt.

In die Berechnung des Aktormoments $M_{\mathrm{A}}$ mit Gleichung (4.14) gehen zusätzlich zu den Schätzfehlern des Modells auch die Messfehler der Druck- und Winkelsensoren ein. Durch Einsetzen der maximalen Messfehler von $\pm 0,5^{\circ}$ und $\pm 5 h P a$ ergibt sich Abbildung 4.50. Aus dem maximalen Fehlerbetrag $\left|\Delta M_{\mathrm{A}}\right|$ von $0,0075 \mathrm{Nm}$ ergibt sich bezogen auf das Maximalmoment von 0,6 $\mathrm{Nm}$ ein maximaler relativer Fehler von $\frac{0,0075 \mathrm{Nm}}{0,6 \mathrm{Nm}}=1,2 \%$. 


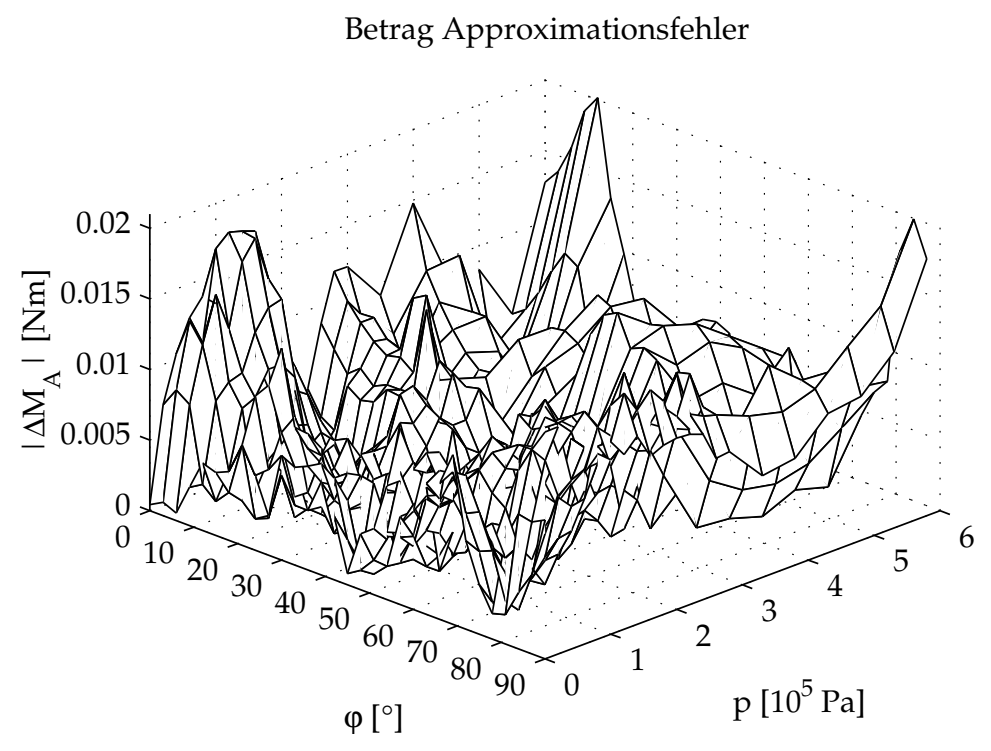

Abbildung 4.49: Momentenschätzfehler durch Aktormodell.

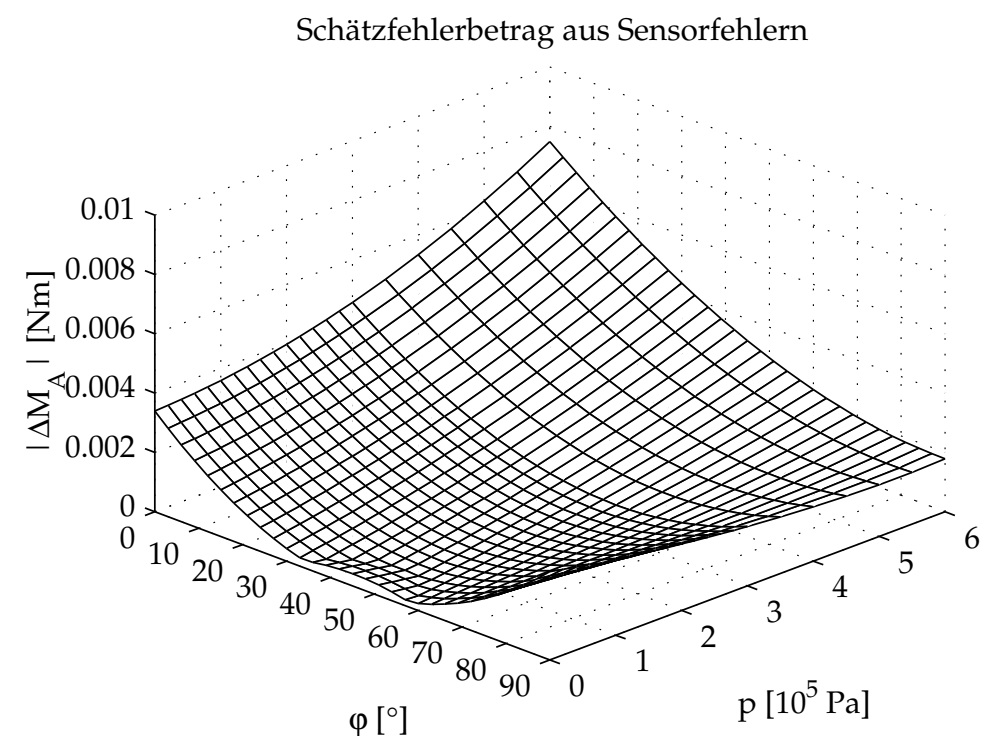

Abbildung 4.50: Momentenschätzfehler durch Winkel- und Druckmessfehler. 


\subsection{Verkabelung}

Die Sensorik einer künstlichen Hand kann nicht losgelöst von der Verkabelung zur Übertragung der Messdaten zu einer Steuerungseinheit betrachtet werden. Nur in wenigen Fällen wie der DLR-Hand II [22] sind integrierte Lösungen zu finden. Meist erfolgt nicht nur die Entwicklung der Sensorausstattung, sondern auch die der Verkabelung erst nach der Fertigstellung der Handmechanik. Eine solche Vorgehensweise führt meist zu unbefriedigenden Lösungen mit außen liegender Verkabelung [84]. In diesem Abschnitt werden die Anforderungen an die Sensorverkabelung zusammengestellt sowie verschiedene Verkabelungsvarianten untersucht.

\subsubsection{Anforderungen}

Wie auch bei der Integration der Sensoren soll für die Leitungen möglichst wenig an der mechanischen Struktur der Finger verändert werden. Daraus lassen sich die folgenden Einzelforderungen ableiten:

- Zum Erhalt der Torsionssteifigkeit der Finger darf die Gelenkachse nicht unterbrochen und der sie umschließende Gelenkbereich nur minimal verändert werden. Die Befestigung des Aktorblechs an Fingerstruktur und Gelenk ist nicht zu schwächen. Die Sicherung gegen das Überstrecken der Gelenke muss erhalten bleiben.

- Die Freigänge Gelenk-Aktor, Fingerstruktur-Aktorverschraubung und die Einbauposition des Gelenkwinkelsensors sind zu beachten. Der Fingerquerschnitt ist nicht $\mathrm{zu}$ vergrößern.

- Wartungsarbeiten an den Fingern sollen nicht erschwert werden. Der Austausch defekter Aktoren und Sensoren soll mit möglichst geringem Aufwand möglich sein.

Für die Verkabelung sind folgende Anforderungen zu beachten:

- Die Leitungsanzahl ist zu minimieren. Dabei sind unterschiedliche Fingerlängen und -konfigurationen sowie der Einsatz von Gleichteilen zu berücksichtigen.

- Innerhalb des Gelenkbeugebereichs von $-10^{\circ}$ bis $+100^{\circ}$ dürfen die für das Leitungsmaterial zulässigen Biegeradien nicht unterschritten werden.

- Die Leitungen sollen an benachbarten Bauteilen nicht scheuern, ein Einklemmen der Leitungen ist zu verhindern. Die Leitungen sind vor äußeren mechanischen Einflüssen zu schützen. 


\subsubsection{Leitermaterialien und Stecker}

\section{Litzenleiter}

Für den Einsatz in Fingergelenken sind Litzenleiter als Flachband- oder Einzelleitungen interessant. Rundleiter mit mehreren Adern werden nicht betrachtet, da sie durch ihre zusätzliche Außenisolation zwar sehr robust, aber auch steif und schwierig zu konfektionieren sind. Für die Leitungen wird der Leiterdurchmesser und bei Litzen zusätzlich der Aufbau angegeben, $7 \times 0,13 \mathrm{~mm}$ steht für sieben Einzeldrähte mit je 0,13 $\mathrm{mm}$ Durchmesser.

Flachbandleitungen werden meist für die parallele Verkabelung innerhalb von Gehäusen verwendet. Das am weitesten verbreitete Rastermaß ist $1,27 \mathrm{~mm}$, bei höheren geforderten Packungsdichten wird ein feineres Rastermaß (fine pitch) von $0,63 \mathrm{~mm}$ verwendet. Im Standardrastermaß sind auch hochflexible Leitungen mit erhöhter Dauerbiegefestigkeit verfügbar [2, 3, 5].

Fine-Pitch-Leitungen werden meist nur bei beengten Anwendungen mit sehr vielen Leitern eingesetzt. Die Einzelleiter sind oft massiv ausgeführt $(1 \times 0,25 \mathrm{~mm})$, mehradrige Typen $(7 \times 0,1 \mathrm{~mm})$ sind aber auch erhältlich [1, 4, 31]. Schneid-KlemmSteckersysteme sind nur für Leitungen mit großer Kontaktzahl $(\geq 10)$ verfügbar.

Die Verwendung von hochflexiblen Einzelleitungen erlaubt den Aufbau von Leitungssätzen ohne überflüssige Leitungen. Bei feinstdrähtiger Verseilung sind die Leiter aus $26 \times 0,05 \mathrm{~mm}$ Adern aufgebaut. Sie erreichen mit Isolation Außendurchmesser von $0,9 \ldots 1,0 \mathrm{~mm}$. Geeignete Steckersysteme mit Crimpkontakten erreichen Rastermaße bis zu 0,8 $\mathrm{mm}$ [87].

\section{Folienleiter}

Die Leitermaterialien dieser Gruppe sind schichtweise aus Kupfer- und Kunststofffolien aufgebaut. Zu unterscheiden ist zwischen FBC (flexible bulk cable), FFC (flexible flat cable) und FPC (flexible printed circuitry, Flexible Leiterplatten), die wichtigsten Kenndaten sind in Tabelle 4.2 zusammengefasst.

FBC und FFC werden mit geraden, parallel geführten Streifenleitern hergestellt. FBC wird als Endlosware gefertigt, Ablängen und Konfektionierung mit Crimpkontakten erfolgen erst nachträglich. Ein Anwendungsbeispiel im Automotive-Bereich ist die Kontakteinheit für den Fahrerairbag im Lenkrad.

FFC werden mit nach Kundenwunsch festgelegter Leiteranzahl, Leiterbreite, Länge, Flexibilität und für das gewählte Kontaktierungsverfahren geeigneten Enden gefertigt [10]. Abgeschirmte Ausführungen sind steifer und nicht dauerbiegefest. Eingesetzt werden FFC z.B. für Verbindungen zum Druckkopf von Tintenstrahldruckern oder zur beweglichen Optikeinheit von CD- und DVDLaufwerken.

Die größten Freiheiten bietet FPC, da fast beliebig geformte Leiterbahnstrukturen realisiert werden können. Ihre Herstellung erfolgt wie bei starren Platinen durch Belichten und Ätzen. Multilayer-Ausführungen, Versteifungen aus verschiedenen Metallen und Kunststoffen, Bonden von Halbleitern sind möglich. Extrem flexible einlagige FPC mit hoher Wärmefestigkeit werden in Festplatten für die 


\begin{tabular}{l|l|l|l|} 
Bezeichnung & $\begin{array}{l}\text { FBC } \\
\text { flexible bulk cable }\end{array}$ & $\begin{array}{l}\text { FFC } \\
\text { flexible flat cable }\end{array}$ & $\begin{array}{l}\text { FPC flexible } \\
\text { printed circuitry }\end{array}$ \\
\hline min. Gesamtdicke & $0,3 \mathrm{~mm}$ & $0,3 \mathrm{~mm}$ & $0,05 \mathrm{~mm}$ \\
\hline min. Leiterdicke & $76 \mu \mathrm{m}$ & $25 \ldots 35 \mu \mathrm{m}$ & $9 \mu \mathrm{m}$ \\
\hline min. Leiterbreite & $0,66 \mathrm{~mm}$ & $0,2 \ldots 0,3 \mathrm{~mm}$ & $0,1 \mathrm{~mm}$ \\
\hline min. Leitungsraster & $1,27 \mathrm{~mm}$ & $0,3 \ldots 0,5 \mathrm{~mm}$ & $0,2 \mathrm{~mm}$ \\
\hline Mindestlänge & $\begin{array}{l}\text { Rollenware, } \\
\text { zugeschnitten }\end{array}$ & $40 \mathrm{~mm}$ & beliebig \\
\hline Folienmaterial & Polyester & $\begin{array}{l}\text { Polyester, } \\
\text { Polyimid }\end{array}$ & Polyimid \\
\hline Herstellung & laminieren & laminieren & laminieren, ätzen \\
\hline Kontaktierung & crimpen & stecken, löten & $\begin{array}{l}\text { stecken, löten, } \\
\text { crimpen }\end{array}$ \\
\hline
\end{tabular}

Tabelle 4.2: Vergleich von Folienleitertypen.

Verbindung zwischen den Schreib-Lese-Köpfen und der Laufwerkselektronik eingesetzt [144]. Für FFC und FPC exisitieren mehrere kompakte Steckersysteme mit unterschiedlichen Rastermaßen und Kontaktanzahlen.

\section{Stromaufnahme und Leitungsquerschnitte}

Die Mindestquerschnitte der Leiter sind beim Maximalfall von vier Winkelsensoren mit einer Stromaufnahme von je $10 \mathrm{~mA}$ und vier FSR-Sensoren mit einem Mindestwiderstand von $20 \mathrm{k} \Omega$ auf der Basis eines Maximalstroms von $41 \mathrm{~mA}$ abzuschätzen. Zu betrachten ist die Verfälschung von Messwerten durch den Spannungsabfall in den Leitungen sowie bei extrem dünn ausgelegten Leitungen die Erwärmung gegenüber der Umgebungstemperatur.

Bei flexiblen Leiterplatten ergibt sich bei der dünnsten üblichen Kupferbeschichtung von $9 \mu \mathrm{m}$ und der Mindestleiterbreite von $100 \mu \mathrm{m}$ auf der mit $20 \mathrm{~cm}$ angenommenen Leitungslänge ein Widerstand $R$ von

$$
R=\varrho_{\mathrm{Cu}} \cdot \frac{l}{A}=17 \cdot 10^{-9} \Omega m \cdot \frac{0,2 m}{0,9 \cdot 10^{-9} m^{2}}=3,78 \Omega .
$$

Hieraus ergibt sich Spannungsabfall von $159 \mathrm{mV}$, der als systematischer Messfehler auszugleichen ist. Die Spannungsversorgung der Sensoren sollte daher mit einer größeren Leiterbreite ausgeführt werden, insbesondere auch um unerwünschter Leitererwärmung vorzubeugen [130].

FFC-Leitungen bieten wegen der größeren Mindestmaße (Kupferdicke $25 \mu \mathrm{m}$, Leiterbahnbreite $0,3 \mathrm{~mm}$ ) eine etwa fünfmal größere Mindestquerschnittsfläche. Ein Litzenleiter aus 26 Einzeladern mit je $50 \mu \mathrm{m}$ Durchmesser bietet mit 0,05 $\mathrm{mm}^{2}$ eine nochmals um den Faktor 7 größere Querschnittsfläche. Messfehler und mögliche Erwärmung sind in diesen Fällen vernachlässigbar. 


\section{Biegeradien}

Die durch das Herstellungsprinzip gerade ausgeführten FFC dürfen zur Realisierung anderer Geometrien auch geknickt und gefaltet werden. Die Anzahl der Knickzyklen ist dabei je nach Ausführung auf 20-50 begrenzt [181].

Die zulässigen Biegeradien für flexible Leiterplatten werden aus der Lagenanzahl bestimmt, für einlagige FPC gilt die sechsfache Dicke, für doppellagige FPC die 12-fache und für mehr Lagen die 24-fache Materialstärke als Mindestbiegeradius [131, 132]. Die Anzahl der in diesem Falle zulässigen Biegezyklen ist sehr gering. Genaue Aussagen werden nicht getroffen.

Als Mindestbiegeradius der meisten Litzen und Flachbandleitungen wird der 8- bis 10-fache Leitungsdurchmesser angegeben. Dabei hängt die Anzahl der maximal erreichbaren Biegezyklen vom Leiteraufbau und der Wahl des Leitermaterials ab.

\section{Biegezyklen}

Tabelle 4.3 führt die zulässigen Mindestbiegeradien unterschiedlicher Leiter auf. Die Werte beziehen sich, soweit vom Hersteller angegeben, auf $10^{6}$ Biegezyklen. Bei Flachbandleitungen kann bei einer Verdoppelung des Biegeradius mit einer etwa zehnmal höheren Anzahl an Biegezyklen gerechnet werden [5]. Unabhängig vom Typ sind die Verbindungsleitungen in einem größtmöglichen Radius über das Gelenk zu führen.

\begin{tabular}{|c|c|c|c|}
\hline Leitungstyp & Bezeichnung & $\begin{array}{l}\text { Biege- } \\
\text { Radius }\end{array}$ & $\begin{array}{l}\text { Zyklen- } \\
\text { anzahl }\end{array}$ \\
\hline \multirow{3}{*}{ 3M Flachbandkabel [5] } & 3365 & $22 \mathrm{~mm}$ & $10^{6}$ \\
\hline & $3539(19 \times 0,08 \mathrm{~mm})$ [2] & $16 \mathrm{~mm}$ & $10^{6}$ \\
\hline & $3319(19 \times 0,08 \mathrm{~mm})[3]$ & $10 \mathrm{~mm}$ & $10^{6}$ \\
\hline \multirow[t]{5}{*}{ Axon Axojump FFC [10] } & Standard $100 \mu m$ & $5 \mathrm{~mm}$ & $6 \cdot 10^{3}$ \\
\hline & Flexibel $50 \mu m$ & $5 \mathrm{~mm}$ & $30 \cdot 10^{3}$ \\
\hline & Superflexibel & $5 \mathrm{~mm}$ & $200 \cdot 10^{3}$ \\
\hline & Extraflexibel $35 \mu \mathrm{m}$ & $5 \mathrm{~mm}$ & $900 \cdot 10^{3}$ \\
\hline & Ultraflexibel $25 \mu \mathrm{m}$ & $5 \mathrm{~mm}$ & $70 \cdot 10^{6}$ \\
\hline \multirow{2}{*}{$\begin{array}{l}\text { Sumitomo Sumi-Card FFC } \\
0,5 / 0,8 \mathrm{~mm} \text { Raster [181] }\end{array}$} & Flexibel $50 \mu \mathrm{m}$ & $15 \mathrm{~mm}$ & $8 \cdot 10^{6}$ \\
\hline & Superflexibel $35 \mu \mathrm{m}$ & $15 \mathrm{~mm}$ & $15 \cdot 10^{6}$ \\
\hline \multirow[t]{3}{*}{ Fujikura FFC [58] } & $100 \mu m$ & $14 \mathrm{~mm}$ & $10^{6}$ \\
\hline & $50 \mu \mathrm{m}$ & $7 \mathrm{~mm}$ & $10^{6}$ \\
\hline & $35 \mu \mathrm{m}$ & $5 \mathrm{~mm}$ & $10^{6}$ \\
\hline Fujikura R/W FPC [144] & $18,5 \mu m$ bei $80^{\circ} \mathrm{C}$ & $2,5 \mathrm{~mm}$ & $10 \cdot 10^{6}$ \\
\hline Nitto High Flex FPC [42] & $25 \mu \mathrm{m}$ bei $80^{\circ} \mathrm{C}$ & $3 \mathrm{~mm}$ & $10^{6}$ \\
\hline
\end{tabular}

Tabelle 4.3: Minimal zulässige Biegeradien für $10^{6}$ Biegezyklen mit verschiedenen Leitermaterialien. 


\section{Auswahl}

Von den Folienleitern sind FPC die interessanteste Variante, da sie die besten Biegeeigenschaften in Kombination mit maximaler Gestaltungsfreiheit bieten. Sie ermöglichen zusätzlich die Integration von Verkabelung, Elektronik und Sensoren zu einer Einheit. Erlaubt die Fingergeometrie gerade ausgeführte Leiter, so sind auch FFC in Betracht zu ziehen.

Für den Fall der von flexiblen Fluidaktoren angetriebenen Finger sind Folienleiter wegen ihrer Knickempfindlichkeit nicht zu empfehlen, da die Komponentenanordnung keinen ausreichenden Schutz erlaubt. Unter den Litzenleitern ist die Einzelverkabelung mit Steckern zu empfehlen, da sie die größte Freiheit beim Entwurf der Leitungssatzstruktur gibt. Für Busstrukturen ist hochflexibles Flachbandkabel wegen seiner mit Schneid-Klemm-Steckern einfachen Konfektionierung interessant.

\subsubsection{Verkabelungsstruktur}

\section{Fingervarianten und Sensorausstattung}

$\mathrm{Zu}$ betrachten sind vier Varianten: Finger mit zwei und drei Gelenken zur Flexion sowie Daumen mit jeweils einem zusätzlichen Gelenk zur Opposition (Abbildung 4.51). Abduktion und Adduktion der Finger werden nicht betrachtet, da die Beugewinkel deutlich kleiner als bei der Flexion sind und die Platzverhältnisse im Bereich der Handfläche als günstiger einzuschätzen sind.

Die Finger sind mit den bereits vorgestellten Winkel- und Kraftsensoren ausgestattet. Die Drucksensoren werden hier nicht betrachtet, da sie außerhalb der Finger im Bereich der Ventilbank angebracht sind. Beim Daumen sind weitere Randbedingungen zu beachten, da das zweite Daumenglied zur Realisierung der Oppositionsstellung in seiner Längsachse um $90^{\circ}$ verdreht ist. Zusätzlich ist das distal hiervon liegende Glied beim Daumen mit vier Gelenken in minimaler Länge ausgeführt. In keinem dieser Daumenglieder erfolgt eine Kraftmessung. Am gedrehten Glied des dreigelenkigen Daumens ist die Winkelmessung von der distalen Seite aus realisiert.

Die Anzahl der für die Sensoranbindung notwendigen Leitungen hängt grundlegend von der gewählten Übertragungsart ab: bei der analogen Übertragung werden die Signale über einen zentralen Multiplexer der A/D-Wandlung zugeführt; andernfalls erfolgt die Digitalisierung dezentral bereits in den Fingergliedern, die Daten werden über ein Bussystem zur Steuerungseinheit übertragen [44].

Zur Energieversorgung ist für die Winkelsensoren Versorgungsspannung und Masse erforderlich. Für die FSR-Sensoren ist die Versorgungsspannung alleine ausreichend, da die Masseanbindung über die Signalleitung und den außerhalb des Fingers gelegenen Spannungsteilerwiderstand erfolgt. 
Finger

2 DOF

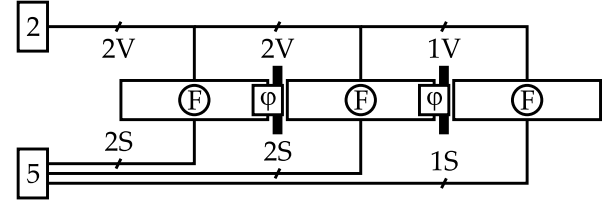

(F) Kraftsensor

$\varphi$ Winkelsensor

$\mathrm{nV}$ Versorgungsleitungen

nS Signalleitungen

Finger

$3 \mathrm{DOF}$

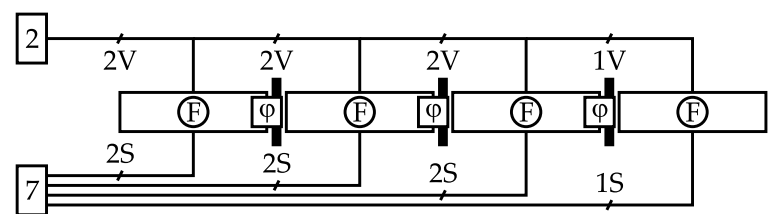

Daumen

$3 \mathrm{DOF}$

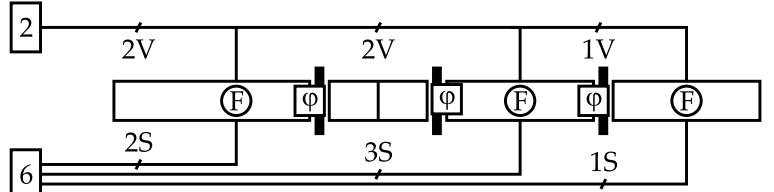

Daumen

$4 \mathrm{DOF}$

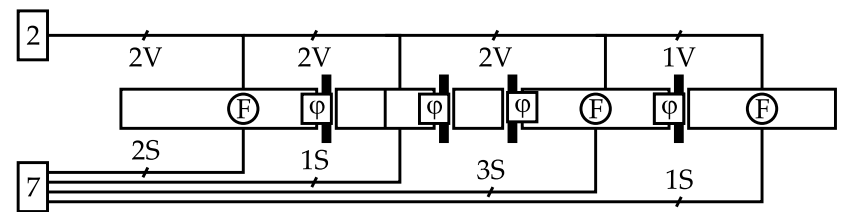

Abbildung 4.51: Fingervarianten: Finger mit zwei und drei Gelenken zur Flexion sowie Daumen mit jeweils einem zusätzlichen Gelenk zur Opposition. DOF=degrees of freedom, Freiheitsgrade.

\section{Analoge Übertragung}

Die Anzahl der über die einzelnen Gelenke zu führenden Leitungen ist in Tabelle 4.4 zusammengefasst. Sie ergibt sich aus Abbildung 4.51 und ist unabhängig von der genauen Leitungssatzstruktur.

\begin{tabular}{l|l|l||l|l|l|l|l|} 
Finger & DOF & Sensoren & Leitungen & \multicolumn{4}{|c}{ Gelenk } \\
& & & zum & \multicolumn{2}{|c|}{ proximal } \\
& & Finger & 1 & 2 & 3 & 4 \\
\hline Finger & 2 & 5 & 7 & 5 & 2 & - & - \\
Finger & 3 & 7 & 9 & 7 & 5 & 2 & - \\
\hline Daumen & 3 & 6 & 8 & 6 & 6 & 2 & - \\
Daumen & 4 & 7 & 9 & 7 & 6 & 6 & 2 \\
\hline
\end{tabular}

Tabelle 4.4: Leitungsverbindungen der Fingervarianten aus Abbildung 4.51. 


\section{Digitale Übertragung}

Wird die Übertragung durch einen Datenbus realisiert, so sind bei einem ${ }^{2} \mathrm{C}$-Bus oder LIN-Bus über jedes Gelenk drei Leitungen zu führen. Eine Verbindung mit SPI-Bus erfordert mindestens vier Leitungen und zusätzlich für jeden am Bus angeschlossenen Teilnehmer ein Auswahlsignal. So ergibt sich beim proximalen Gelenk des dreigelenkigen Fingers und des viergelenkigen Daumens eine maximale Reduktion von 7 auf 3 Leitungen. Ihr steht der Platzbedarf für die in den Fingergliedern unterzubringende Elektronik gegenüber.

Solange die Fingerspitzen mit einem einzelnen Sensor ausgestattet sind, ergibt sich an den distalen Gelenken sogar ein Mehrbedarf an Leitungen. Da viele kompakte Steckersysteme das Anschlagen mehrerer Leitungen an einem Steckerkontakt nicht erlauben, ist auch die Ersparnis an Steckerbauraum bei einem Bussystem im Finger nur minimal.

\subsubsection{Leitungssatzoptimierung}

\section{Randbedingungen}

Für das Layout eines Leitungssatzes mit Einzelleitungen und Steckverbindern sind folgende Randbedingungen zu beachten:

- Kompakte Steckerbauformen erlauben nur eine Leitung je Steckerkontakt, also keine Mehrfachanschläge.

- Die Spannungsversorgung soll als Kette, also nur mit Verbindungen zu direkt benachbarten Fingergliedern ausgeführt werden. Die Sensorsignale sollen mit einer sternförmigen Leitungsstruktur möglichst direkt zur Handelektronik geleitet werden.

- Von Fingerwurzel zur Handelektronik und über das distale Gelenk zur Fingerspitze soll nur je ein Leitungsstrang weggeführt werden. Seitenwechsel von Leitungen sind nicht zulässig.

- Leitungen und Steckverbinder sind möglichst gleichmäßig auf beiden Seiten des Fingers zu verteilen. So werden über jedes Gelenk statt einem dicken zwei dünne Leitungsstränge geführt, womit eine größere Anzahl an Biegezyklen erreicht werden kann. Außerdem erlauben zwei kleine statt einem großen Stecker in jedem Fingerglied kürzere Fingerglieder.

- Am gedrehten Daumenglied soll nur ein Stecker für den Anschluss eines Winkelsensors angebracht werden. Ansonsten sind redundante Leitungen zu vermeiden.

\section{Variantenerzeugung}

Aus den Leitungsanzahlen in Tabelle 4.4 und den Randbedingungen des vorangegangenen Abschnitts lassen sich für die Fingerkonfigurationen der Abbildung 4.51 
verschiedene Leitungssatzvarianten erzeugen. Die in den folgenden Abschnitten beschriebenen Leitungssatzstrukturen werden beispielhaft in den Abbildungen 4.52 und 4.53 illustriert.

Die Variante a) ist ein Gleichteileansatz, bei dem die Leitungen abwechselnd auf beiden Fingerseiten geführt werden. Für jedes Gelenk wird ein separater Leitungssatz verwendet. Die Stecker sind auf den Platinen so angeordnet, dass alle Leitungssätze unabhängig von ihrer Leitungszahl mit identischer Länge ausgeführt sind.

Variante b) ist ein auf die minimale Kontaktanzahl optimierter einteiliger Leitungssatz. Er nimmt keine Rücksicht auf die Forderungen nach Leitungs- und Kontaktsymmetrie. Die Varianten c) bis f) sind hiervon abgeleitet, auf Kosten der Kontaktanzahl wird eine höhere Leitungs- und Kontaktsymmetrie erzeugt.

In Variante c) wird die Spannungsversorgung auf die bisher freie Fingerseite gelegt. Variante d) ergänzt dies um die Leitungen für den Kraftsensor an der Fingerspitze. Bei der Variante e) ist das Sensorsignal zusammen mit der Spannungsversorgung bis an die Fingerbasis geführt. Variante f) ist ein von Variante a) abgeleiteter Gleichteileansatz, bei dem die Leitungen auf beiden Gelenkseiten möglichst gleichmäßig verteilt sind.

\section{Vergleichskriterien}

Die Vergleichskriterien für die Varianten werden aus den gesetzten Randbedingungen hergeleitet. Sie verwenden die Kontaktanzahlen $k_{\mathrm{L}}, k_{\mathrm{R}}$ und die Leitungszahlen $l_{\mathrm{L}}, l_{\mathrm{R}}$ an jedem Fingerglied bzw. Gelenk $j$. Die Indizes $L$ und $R$ bezeichnen jeweils die linke und rechte Fingerseite. Gezählt wird von $j=0$ am Steckverbinder zwischen der Elektronik und dem ersten Fingerglied bis zum durch die Gelenkanzahl $n$ bestimmten Maximum. Ein Teil der Kriterien bewertet zudem distal liegende Leitungen und Kontakte stärker als proximal liegende. Hierzu wird der Gewichtungsfaktor $w^{j}$ mit $w=2$ herangezogen. Die zu bevorzugenden Lösungen erhalten bei allen Bewertungskriterien kleine Zahlenwerte.

Die Kontaktanzahl $n_{\mathrm{K}}$ in Gleichung (4.16) ist die Gesamtzahl der für die Verkabelung benötigten Kontakte.

$$
n_{\mathrm{K}}=\sum_{j=0}^{n+1}\left(k_{\mathrm{L} j}+k_{\mathrm{R} j}\right)
$$

Die Verteilung der Kontakte $v_{\mathrm{K}}$ in Gleichung (4.17) ist die mit dem Faktor $w^{j}$ gewichtete Kontaktzahl. Durch die Gewichtung werden distal liegende Kontakte stärker als proximal liegende bewertet.

$$
v_{\mathrm{K}}=\sum_{j=0}^{n+1}\left(\left(k_{\mathrm{L} j}+k_{\mathrm{R} j}\right) \cdot w^{j}\right)
$$

Die Kontaktsymmetrie $s_{\mathrm{K}}$ in Gleichung (4.18) ist ein Maß, das die symmetrische Anordnung von Kontakten bevorzugt, geringe Differenzen zwischen den beiden 
Fingerseiten erlauben bei gleicher Kontaktanzahl kürzere Fingerglieder. Die distal liegenden kürzeren Fingerglieder werden mit dem Faktor $w^{j}$ stärker gewichtet.

$$
s_{\mathrm{K}}=\sum_{j=1}^{n}\left(\left|k_{\mathrm{L} j}-k_{\mathrm{R} j}\right| \cdot w^{j}\right)
$$

Die Leitungssymmetrie $s_{\mathrm{L}}$ in Gleichung (4.19) ist die mit $w^{j}$ gewichtete Differenz der auf beiden Seiten des Gelenks geführten Leitungen. Da die Fingerzuführungsleitung und die Leitungen über das distale Gelenk asymmetrisch auszuführen sind, werden sie von dieser Bewertung ausgenommen.

$$
s_{\mathrm{L}}=\sum_{j=1}^{n-1}\left(\left|l_{\mathrm{L} j}-l_{\mathrm{R} j}\right| \cdot w^{j}\right)
$$

Die Fingerlänge $l_{\mathrm{F}}$ in Gleichung (4.20) ist ein Maß für die durch die Steckeranordnung bedingte zusätzliche Länge gegenüber einem sensorlosen Finger mit minimalen Gliederlängen. Ausgehend von einreihigen Kontakten in einem Raster von $1 \mathrm{~mm}$ wird für alle Fingerglieder die jeweils größere der beiden Kontaktzahlen auf beiden Seiten aufsummiert.

$$
l_{\mathrm{F}}=\sum_{j=0}^{n} \max \left(k_{\mathrm{L} j}, k_{\mathrm{R} j}\right)
$$

Die Leitungsanzahl $n_{\mathrm{L}}$ in Gleichung (4.21) gibt die Anzahl der über die Gelenke zu führenden Leitungsabschnitte wieder. Sie ist durch die in Tabelle 4.4 vorgegebene Verkabelungsstruktur für alle Varianten gleich und damit nicht zum Vergleich geeignet.

$$
n_{\mathrm{L}}=\sum_{j=0}^{n}\left(l_{\mathrm{L} j}+l_{\mathrm{R} j}\right)
$$

\section{Gewichtung der Vergleichskriterien}

Durch paarweisen Vergleich wird in Tabelle 4.5 eine Gewichtung $g$ für die fünf Vergleichskriterien ermittelt. Die beiden wichtigsten Kriterien Kontaktanzahl $n_{\mathrm{K}}$ und Fingerlänge $l_{\mathrm{F}}$ beeinflussen danach insgesamt $60 \%$ der Gesamtbewertung $b$ eines Lösungsansatzes. Den geringsten Einfluss von zusammen nur $20 \%$ haben die Kontaktverteilung $v_{\mathrm{K}}$ und die Kontaktsymmetrie $s_{\mathrm{K}}$.

Die Gesamtbewertung $b$ wird aus der mit $g$ gewichteten Summe der auf den Wertebereich $0 \ldots 1$ normierten Einzelergebnisse berechnet. Sie ist also für eine Lösung, die bei allen Kriterien das beste Ergebnis liefert, 0; im entgegengesetzten Fall 1. Zur besseren Übersicht sind in den Bewertungstabellen sehr gute Einzelergebnisse mit ' ++ ', mittlere mit ' 0 ' und sehr schlechte mit '- -' markiert.

Bewertet werden hier exemplarisch der Finger mit 3 Gelenken und der Daumen mit 4 Gelenken. Für die kürzeren Varianten wird auf den Anhang, Abschnitt 7.2 verwiesen. 


\begin{tabular}{ll|c|c|c|c|c} 
Vergleichskriterium & & $n_{\mathrm{K}}$ & $v_{\mathrm{K}}$ & $s_{\mathrm{K}}$ & $s_{\mathrm{L}}$ & $l_{\mathrm{F}}$ \\
\hline Kontaktanzahl & $n_{\mathrm{K}}$ & 1 & 0 & 1 & 0 & 2 \\
Kontaktverteilung & $v_{\mathrm{K}}$ & 2 & 1 & 1 & 2 & 2 \\
Kontaktsymmetrie & $s_{\mathrm{K}}$ & 1 & 1 & 1 & 2 & 2 \\
Leitungssymmetrie & $s_{\mathrm{L}}$ & 2 & 0 & 0 & 1 & 2 \\
Fingerlänge & $l_{\mathrm{F}}$ & 0 & 0 & 0 & 0 & 1 \\
\hline Punkte & $\sum=25$ & 6 & 2 & 3 & 5 & 9 \\
\hline Gewicht $g$ & $\sum=1$ & 0,24 & 0,08 & 0,12 & 0,20 & 0,36 \\
\hline
\end{tabular}

Tabelle 4.5: Gewichtungstabelle für Vergleichskriterien: '0'-Zeile wichtiger als Spalte, '1'-gleich wichtig, '2'-Spalte wichtiger als Zeile.

\subsubsection{Fingerleitungssatz}

Die nach der Beschreibung im Abschnitt 4.4.4 hergeleiteten Varianten für den Fingerleitungssatz mit drei Freiheitsgraden sind in Abbildung 4.52 dargestellt.
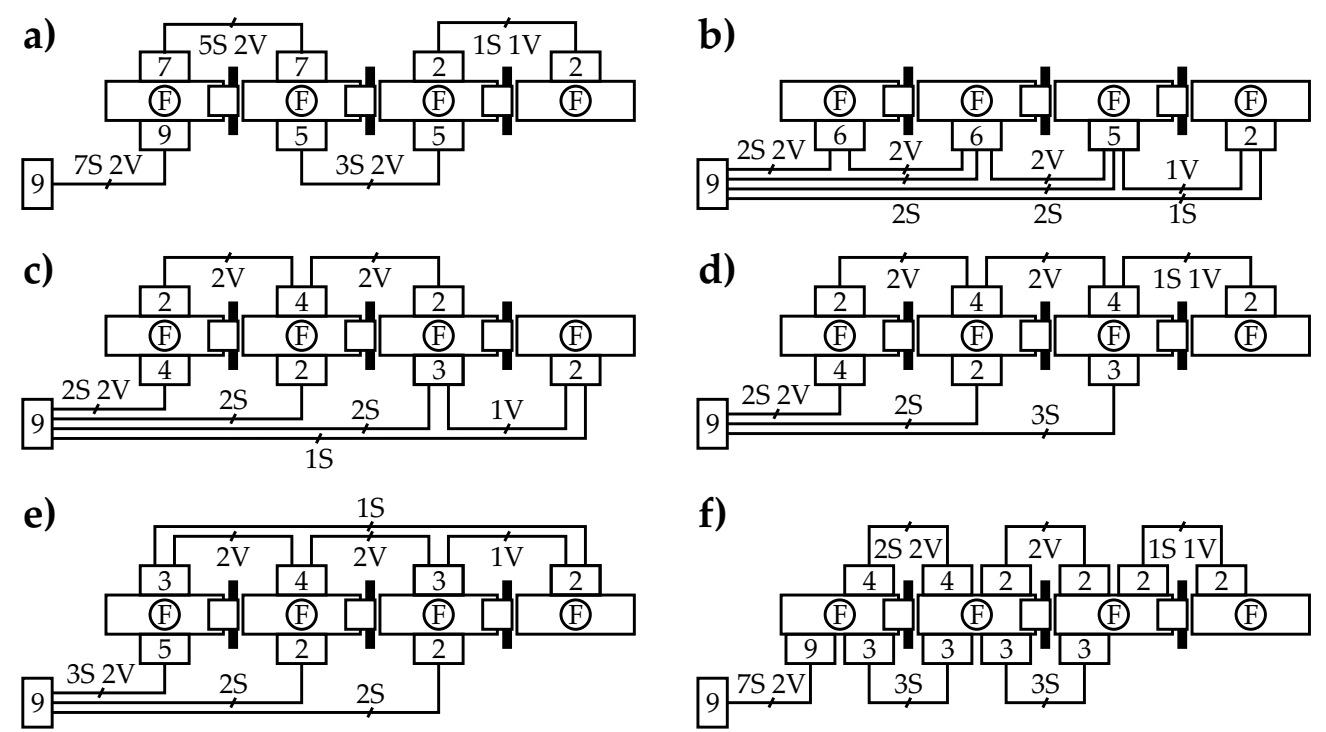

Abbildung 4.52: Leitungssatzvarianten für einen Finger mit drei Gelenken.

Die Bewertungstabelle 4.6 liefert für Variante c) bei allen Kriterien außer der Leitungssymmetrie die besten Ergebnisse, die nachfolgenden Varianten e) und d) schneiden bei Kontaktanzahl und -verteilung schlechter ab. Variante b) wird trotz Bestbewertung bei Kontaktanzahl und -verteilung wegen der schlechten Bewertung der Symmetrien auf Rang 4 gesetzt. Varianten $\mathrm{f}$ ) und a) verlieren wegen ihrer nochmals schlechteren Kontaktanzahl und -verteilung.

Beim kürzeren Finger mit nur zwei Gelenken (Abbildung und Tabelle im Anhang, Abschnitt 7.2) ergibt sich eine weitgehend identische Bewertungsreihenfolge mit vertauschten letzten Plätzen für die Varianten a) und f). Die bestplatzierte Va- 


\begin{tabular}{l|rl|rl|rc|rc|rc|rc|} 
Variante & $\mathrm{a}$ & $\mathrm{b}$ & $\mathrm{c}$ & $\mathrm{d}$ & $\mathrm{e}$ & $\mathrm{f}$ & \\
\hline$n_{\mathrm{K}}$ & 46 & - & 28 & ++ & 28 & ++ & 30 & + & 30 & + & 50 & -- \\
$v_{\mathrm{K}}$ & 177 & - & 117 & ++ & 117 & ++ & 133 & 0 & 121 & + & 195 & -- \\
$s_{\mathrm{K}}$ & 36 & 0 & 76 & - & 20 & ++ & 20 & ++ & 20 & ++ & 34 & + \\
$s_{\mathrm{L}}$ & 34 & - & 34 & - & 10 & + & 10 & + & 6 & ++ & 6 & ++ \\
$l_{\mathrm{F}}$ & 23 & - & 19 & 0 & 13 & ++ & 14 & + & 14 & + & 27 & - \\
\hline$b$ & 0,75 & 0,47 & 0,03 & 0,09 & 0,05 & 0,71 \\
\hline Rang & 6 & 4 & 1 & 3 & 2 & 5 \\
\hline
\end{tabular}

Tabelle 4.6: Bewertung der Leitungssatzvarianten für Finger mit drei Gelenken.

riante c) erhält im Vergleich aller Kriterien die beste Bewertung, also eine Gesamtbewertung von $b=0$. Für die zweitbeste Lösung e) ergibt sich eine kaum bessere Bewertung als für Variante d).

\subsubsection{Daumenleitungssatz}

Der Daumenleitungssatz mit vier Freiheitsgraden unterscheidet sich vom Finger mit drei Gelenken im wesentlichen durch eine Unterteilung des zweiten Fingerglieds in zwei möglichst kurz ausgeführte Daumenglieder (Abbildung 4.53). Dabei ist das proximale um die Fingerlängsachse $90^{\circ}$ gedreht, womit die Oppositionsbewegung des Daumens erreicht wird. Zur Einsparung von Kontakten wird dieses Daumenglied separat angeschlossen. Die Messung des Gelenkwinkels des dritten Gelenks erfolgt von der distalen Seite her, womit auf die Verkabelung des kurzen Daumenglieds verzichtet werden kann.
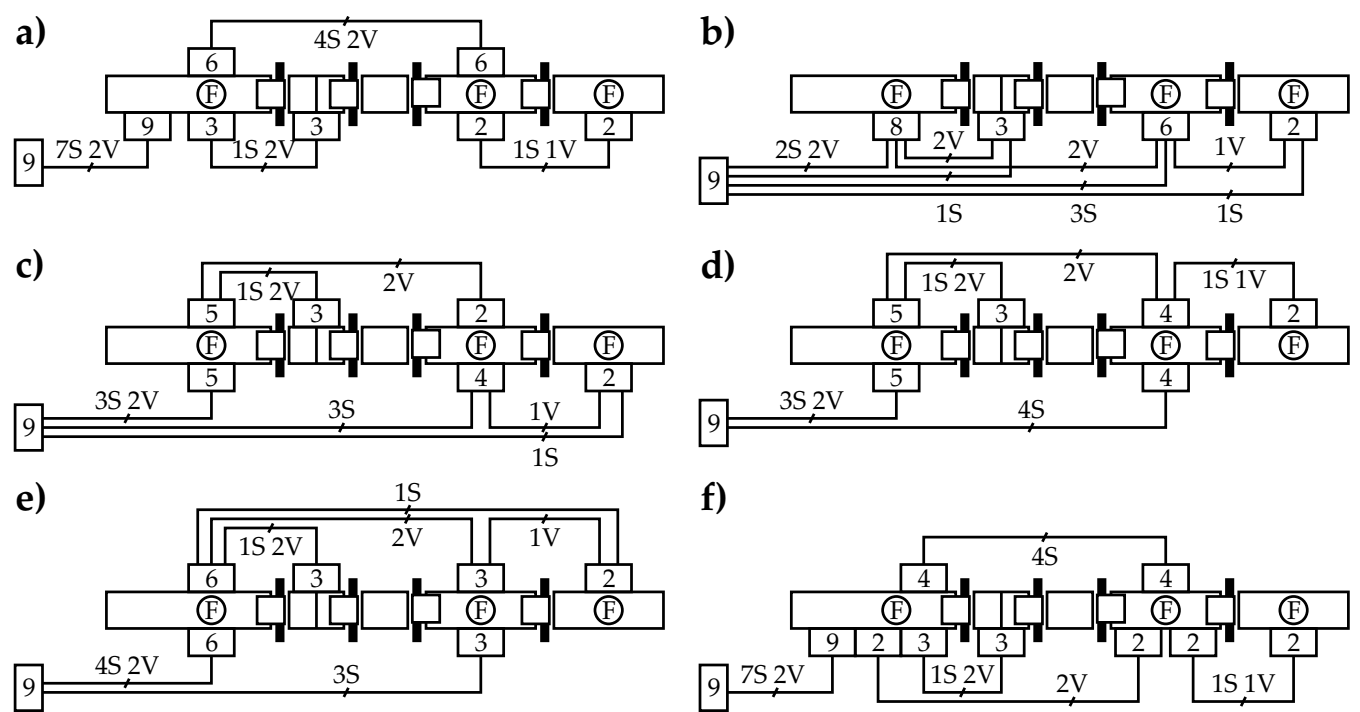

Abbildung 4.53: Leitungssatzvarianten für den Daumen mit vier Gelenken. 


\begin{tabular}{l|rl|rl|rl|rr|rr|rc|} 
Variante & $\mathrm{a}$ & $\mathrm{b}$ & $\mathrm{c}$ & $\mathrm{d}$ & $\mathrm{e}$ & $\mathrm{f}$ & \\
\hline$n_{\mathrm{K}}$ & 40 & - & 28 & ++ & 30 & + & 32 & 0 & 32 & 0 & 43 & -- \\
$v_{\mathrm{K}}$ & 249 & - & 197 & ++ & 201 & + & 233 & - & 205 & 0 & 269 & -- \\
$s_{\mathrm{K}}$ & 88 & - & 124 & -- & 44 & + & 12 & ++ & 12 & ++ & 52 & 0 \\
$s_{\mathrm{L}}$ & 78 & - & 90 & - & 26 & 0 & 26 & 0 & 6 & ++ & 10 & + \\
$l_{\mathrm{F}}$ & 23 & - & 16 & 0 & 11 & ++ & 11 & ++ & 11 & ++ & 20 & - \\
\hline$b$ & 0,77 & 0,47 & 0,12 & 0,15 & 0,07 & 0,73 \\
\hline Rang & 6 & 4 & 2 & 3 & 1 & 5 \\
\hline
\end{tabular}

Tabelle 4.7: Bewertung der Leitungssatzvarianten für Daumen mit 4 Gelenken.

Die Bewertung der Varianten in Tabelle 4.7 führt zu einem anderen Ergebnis als beim Finger mit drei Gelenken. Die Leitungsverlegung ist nach der bisher nur zweitbesten Variante e) auszuführen, da sie bei Fingerlänge, Kontakt- und Leitungssymmetrie die besten Bewertungen erhält. Als zweitbeste Variante ist c) zu empfehlen, die bei der Kontaktanzahl und -verteilung besser als d) ist, bei den Symmetrien jedoch nicht so gut abschneidet. Wie bei den Fingern geht Platz 4 an Variante b), die letzten Plätze teilen sich die Varianten a) und f). Zu diesen ist anzumerken, dass der ursprünglich angedachte Gleichteileansatz hier nur eingeschränkt realisierbar ist, da neben den kurzen Leitungsverbindungen für ein Gelenk auch längere Verbindungen notwendig sind.

Die Daumenvariante mit drei Gelenken kann aus dem Finger mit zwei Freiheitsgraden durch Hinzufügen eines zusätzlichen gedrehten Daumenglieds und einen distal hierzu angeordneten Winkelsensors hergeleitet werden. Ergebnisse für sie sind im Anhang (Abschnitt 7.2) aufgeführt. Die Rangreihenfolge bleibt gegenüber dem Daumen mit vier Freiheitsgraden unverändert, alleine die Bewertung der Variante d) fällt deutlich schlechter als beim viergelenkigen Daumen aus.

\subsubsection{Zusammenfassung}

Hergeleitet wurde eine Reihe von Randbedingungen und Bewertungskriterien für unterschiedliche Varianten zur Verkabelung von Fingern und Daumen unterschiedlicher Gelenkanzahl. Die Leitungssatzvarianten wurden von allgemeinen Leitungssätzen mit optimaler Leitungsanzahl abgeleitet.

Für alle untersuchten Fingervarianten zeigen die Bewertungsergebnisse, dass mit den besonders einfach herleitbaren Gleichteileansätzen der Varianten a) und f), die mehrheitlich aus Leitungsbrücken zu den direkt benachbarten Fingergliedern bestehen, keine guten Ergebnisse erzielt werden. Durch gezieltes Verteilen der Versorgungs- und Signalleitungen auf beiden Fingerseiten wurden unter Berücksichtigung der für die Stecker erforderlichen Mindestfingerlänge und der Kontaktanzahl mit den Varianten c) und e) für alle Fingertypen die besten Ergebnisse erzielt. 


\section{Anwendung der Sensoren: Gelenkregelung und Greifen}

Die Grundlage koordinierter Bewegung und Kraftausübung der Hand ist die Regelung der einzelnen Gelenke unter Verwendung der im vorangegangenen Kapitel beschriebenen Sensoren. In diesem Kapitel wird der Einsatz der Sensoren in der Regelung und Steuerung einzelner und mehrerer Gelenke im pneumatischen und hydraulischen Betrieb demonstriert. Abschließend wird aufgezeigt, wie eine Handsteuerung mit dem Steuerungssystem eines humanoiden Roboters zusammenarbeiten kann.

\subsection{Positionsregelung im pneumatischen Betrieb}

In diesem Abschnitt wird zunächst ein Modell für das Bewegungsverhalten des Gelenks im pneumatischen Betrieb aufgestellt, auf dessen Grundlage eine Positionsregelung und die ihr zu Grunde liegende Druckregelung hergeleitet werden.

\subsubsection{Gelenkmodell}

Das Gelenkmodell beschreibt den sich in Abhängigkeit von Aktordruck $p$ einstellenden Gelenkwinkel $\varphi$. Im statischen Fall $(\dot{\varphi}=0, \ddot{\varphi}=0)$ befindet sich das Aktormoment $M_{\mathrm{A}}(\varphi, p)$ im Gleichgewicht mit dem z.B. von einem Objektkontakt herrührende Störmoment $M_{\text {Stör }}$.

Beim Beugen des Gelenks wirken zusätzlich das sich aus der viskosen Reibung $d$ des Gelenks [6] ergebende geschwindigkeitsproportionale Dämpfungsmoment $d \cdot \dot{\varphi}$ und das Massenträgheitsmoment $J \cdot \ddot{\varphi}$. Hieraus ergibt sich die in Abbildung 5.1 schematisch dargestellte Bewegungsgleichung des Gelenks der Gleichung (5.1):

$$
J \cdot \ddot{\varphi}+d \cdot \dot{\varphi}=M_{\mathrm{A}}(\varphi, p)-M_{\mathrm{Stör}} .
$$

Der Vergleich der bei $M_{\text {Stör }}=0$ von Modell und realer Strecke angenommenen Winkelstellung zeigt zusätzlich eine zeitabhängige Hysterese und ein Kriechverhalten des Aktors (Abbildung 5.2 links).

Die dabei auftretende Verschiebung $\Delta \varphi$ von bis $\mathrm{zu} 4^{\circ}$ wird vom viskoelastischen Verhalten des Aktormaterials verursacht. Bei Beschreibung mit einem nichtlinearen Modell erster Ordnung kann das in Abbildung 5.2 rechts gezeigte 


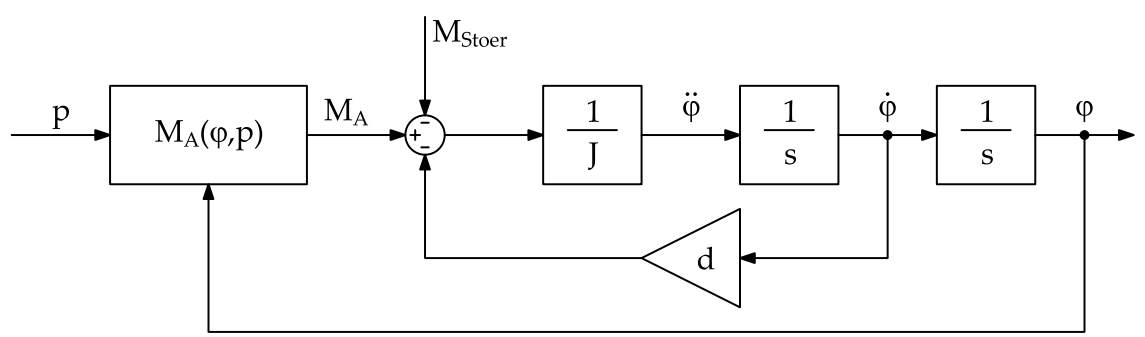

Abbildung 5.1: Modell für das Bewegungsverhalten des Gelenks.
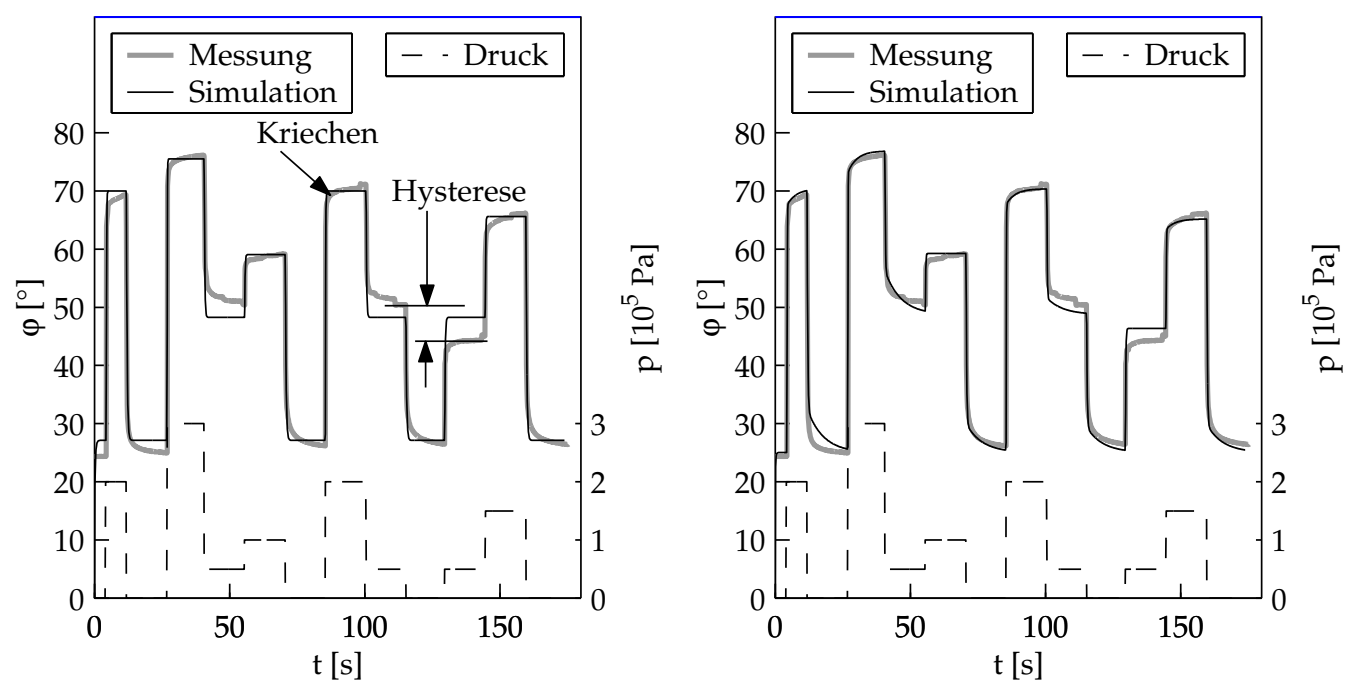

Abbildung 5.2: Bewegungsverhalten des Gelenks in Messung und Simulation. Links ohne, rechts mit Modellierung des viskoelastischen Aktorverhaltens. 
Modellverhalten erreicht werden. Hierzu wurde das Aktormodell zur Form von Gleichung (5.2) erweitert.

$$
M_{\mathrm{A}}(\varphi, p)=a_{00}+a_{01} \cdot(\varphi+\Delta \varphi)+a_{10} \cdot p+a_{11} \cdot \varphi \cdot p
$$

Für eine ausführliche Herleitung und Diskussion dieses Verhaltens wird auf [13] verwiesen.

\subsubsection{Positionsregler}

Für die Regelung der Gelenkposition im pneumatischen Betrieb wird ein kaskadierter Regler mit einer unterlagerten Druckregelung (Abbildung 5.3) verwendet. Dieser Ansatz erlaubt beim Greifen die gezielte Beeinflussung des Aktormoments über den Solldruck.

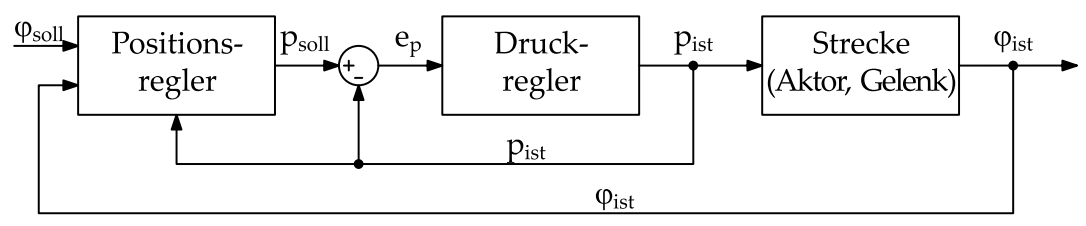

Abbildung 5.3: Schematische Darstellung des pneumatischen Systems mit kaskadierter Positions- und Druckregelung.

Zunächst wird die der Positionsregelung unterlagerte Druckregelung mit einem analog ansteuerbaren Druckregelventil ausgeführt (Abbildung 5.4). Sein Verhalten entspricht einem $\mathrm{PT}_{1}$-Glied. Als Druckversorgung steht ein Kompressor mit Druckbehälter zur Verfügung. Der Arbeitsdruck $p_{V}$ wird durch einen Druckbegrenzer konstant gehalten.

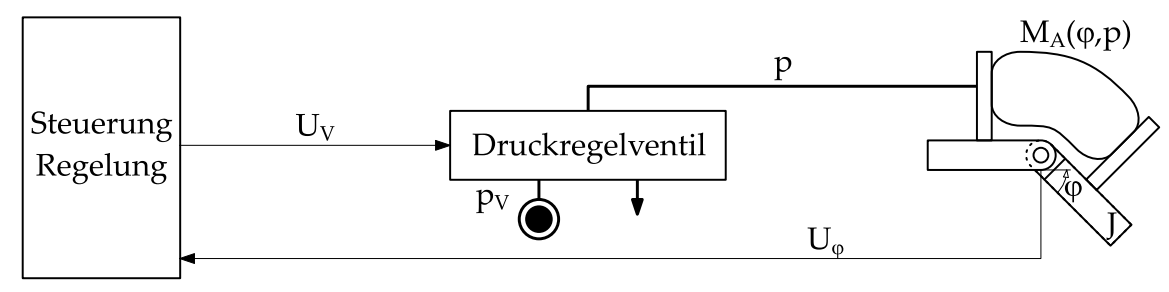

Abbildung 5.4: Schaltbild der pneumatischen Gelenkregelung mit Druckregelventil.

Der zeitdiskrete PID-Positionsregler (Abbildung 5.5) mit der Abtastzeit $T_{\mathrm{A}}$ gibt als Stellgröße den Solldruck $p_{\text {soll }}$ für das Druckregelventil aus. Durch eine Parameteradaption von $k_{\mathrm{R}}, T_{\mathrm{N}}$ und $T_{\mathrm{V}}$ wird das nichtlineare Streckenverhalten ausgeglichen. Als Anti-Reset-Windup Maßnahme sind sowohl Integrator als auch Reglerausgang begrenzt. Durch Führungsgrößensprünge über den D-Anteil verursachte Spitzen in der Stellgröße $p_{\text {soll }}$ werden vermieden, indem nur die Regelgröße $\varphi_{\text {ist }}$ in den D-Anteil eingeht. 


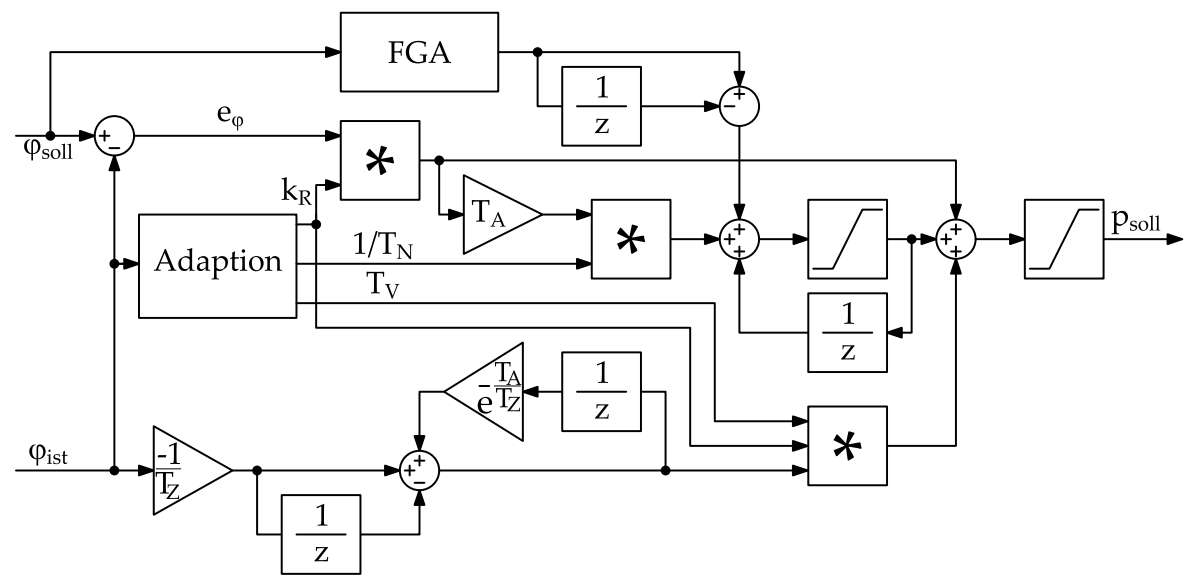

Abbildung 5.5: Interner Aufbau des pneumatischen Positionsreglers.

Zur Verbesserung des Führungsverhaltens wird der Positionsregler um eine Führungsgrößenaufschaltung (FGA) ergänzt, indem der Solldruck über die Nullmomentenkennlinie des Aktorkennfelds aus dem Sollwinkel berechnet wird. Die Ableitung der FGA wird in den begrenzten Integrator des I-Anteils eingespeist.

\section{Messungen}

Für die Messung des Positionsreglerverhaltens wurde ein Eingelenkfinger (Abbildung 5.6) angefertigt. Dieser ist mit einem Winkelsensor und zwei taktilen Sensoren ausgestattet. Der externe Drucksensor ist über einen $20 \mathrm{~cm}$ langen Schlauch angeschlossen. Über ihn erfolgt auch die Verbindung zum Druckregelventil mit einer weiteren $30 \mathrm{~cm}$ langen Schlauchverbindung.

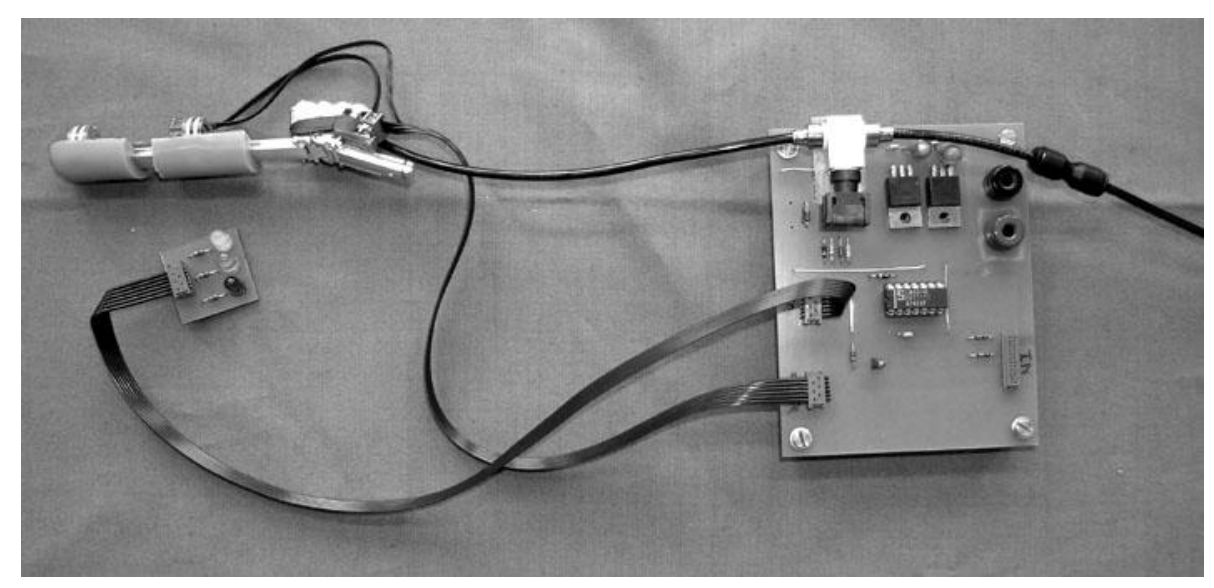

Abbildung 5.6: Eingelenkfinger für den Betrieb des pneumatischen Positionsreglers. 
Als Führungsgröße $\varphi_{\text {soll }}$ (Abbildung 5.7 oben) werden mehrere geglättete Rampenverläufe von $35^{\circ}$ nach $70^{\circ}$ mit den Schließdauern $1,0 s ; 0,7 s ; 0,5 s ; 0,3 s ; 0,2 s$ und der konstanten Öffnungsdauer $0,4 s$ vorgegeben. Die Glättung erfolgt durch einen Tiefpassfilter $\left(\mathrm{PT}_{1}, T_{1}=0,1 s\right)$. Für den gegebenen Führungsgrößenverlauf wird ein mittlerer Betrag des Positionsfehlers von $4,5^{\circ}$ gemessen. Soll- und Istdruck des unterlagerten Druckreglers mit einem mittleren Fehlerbetrag von $0,3 \cdot 10^{5} \mathrm{~Pa}$ werden in Abbildung 5.7 Mitte gezeigt.

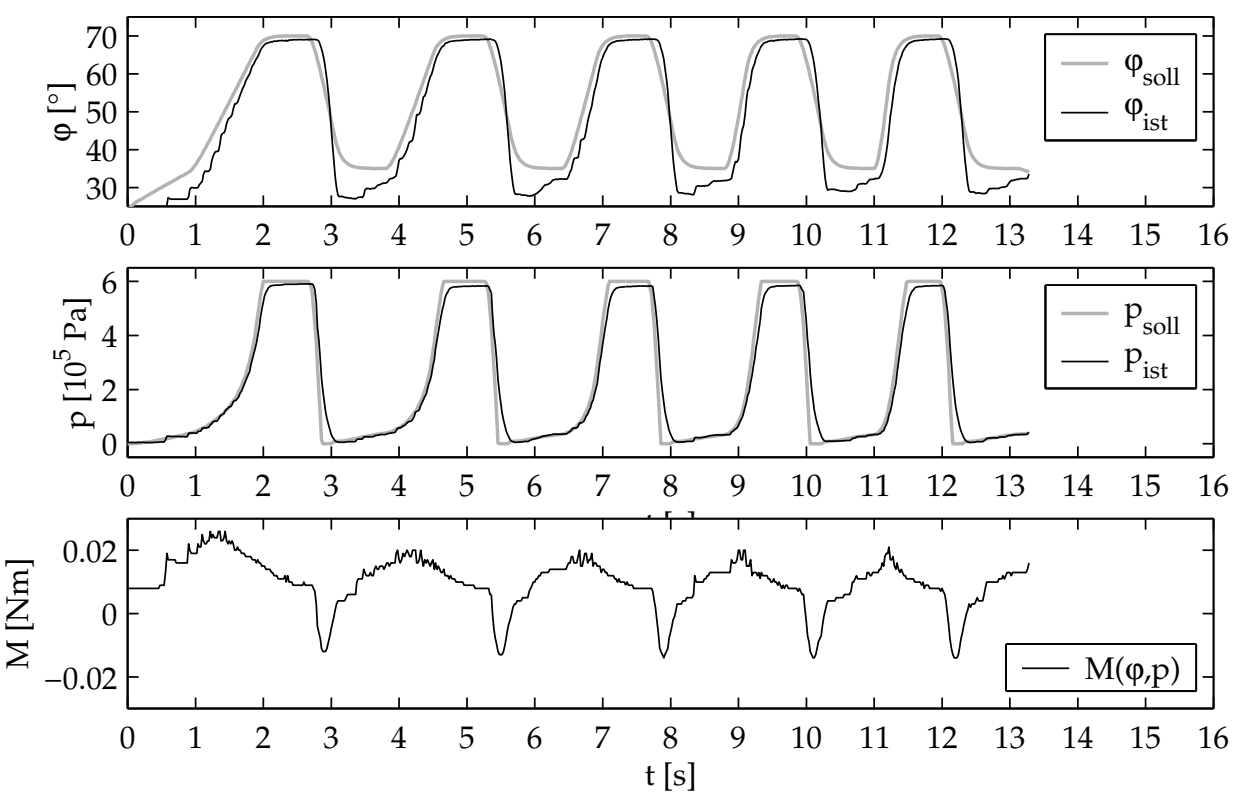

Abbildung 5.7: Messung: Positionsregelung eines Gelenks im pneumatischen Betrieb.

In Abbildung 5.7 unten ist das während der Bewegung aus $\varphi_{\text {ist }}$ und $p_{\text {ist }}$ geschätzte Aktormoment aufgetragen. Deutlich zu erkennen sind die aus der Bewegungsgleichung des Gelenks zu erwartenden Maxima zu Beginn der Schließbewegungen. Ebenso wird bestätigt, dass der Finger zum Erreichen der geforderten Dynamik mit 0,026 $\mathrm{Nm}$ nur etwa $5 \%$ des maximalen Aktormoments von 0,54 $\mathrm{Nm}$ (bei $\varphi=0^{\circ}$ und $p_{A}=6 \cdot 10^{5} \mathrm{~Pa}$ ) benötigt.

\subsubsection{Druckregelung}

Der diskrete Aufbau der Druckregelung (Abbildung 5.8) mit digital ansteuerbaren 2/2-Wege-Ventilen hat gegenüber einem analog anzusteuernden Druckregelventil für den industriellen Einsatz neben der Platzersparnis auch den Vorteil der Ansteuerbarkeit über die Digitalausgänge eines Mikrocontrollers [62]. 


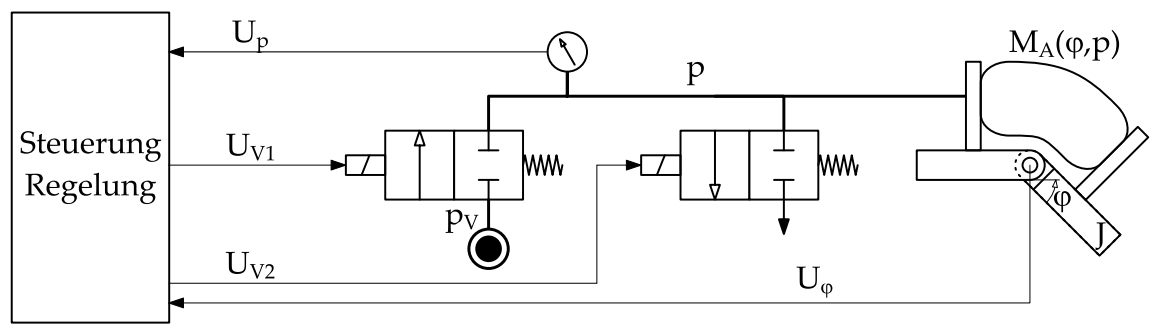

Abbildung 5.8: Pneumatische Gelenkregelung mit diskreter Druckregelung.

\section{Streckenmodell}

Das Modell für die zu regelnde Strecke mit einem zunächst als konstant angenommenen Aktorvolumen (Abbildung 5.9) basiert auf einer Massenstromgleichung für den Ventildurchfluss und der allgemeinen Gasgleichung zur Berechnung des Aktordrucks aus der Gasmasse.

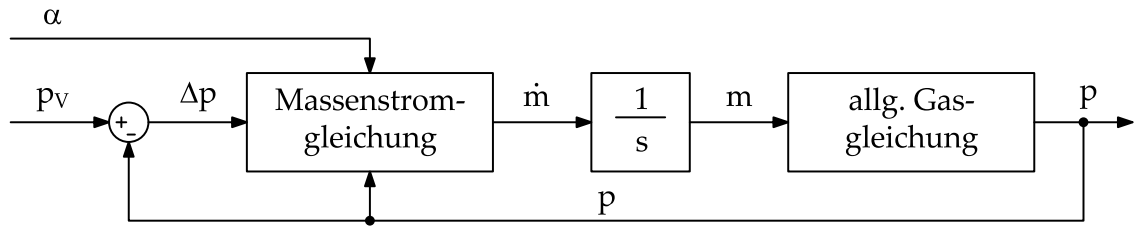

Abbildung 5.9: Streckenmodell für die Druckregelung.

In den Massenstrom $\dot{m}$ des als Blende angenäherten Ventils gehen nach Gleichung (5.3) die Druckdifferenz $\Delta p$, die Dichte $\varrho$ der Luft, die Querschnittsfläche $A$ sowie der mit 0,8 anzunehmende Verlustbeiwert $\mu$ ein. Die Querschnittsfläche $A$ ergibt sich zusätzlich aus der Querschnittsfläche $A_{0}$ des vollständig geöffneten Ventils und dem Öffnungsgrad $\alpha$.

$$
\dot{m}=\mu A \sqrt{2 \varrho \Delta p}=\mu \alpha A_{0} \sqrt{2 \varrho \Delta p}
$$

Da $\varrho$ hier die Dichte auf der Niederdruckseite des Ventils beschreibt, darf nur im Fall des Luftablassens der Umgebungsdruck mit $\varrho=\varrho_{0}=1,29 \frac{\mathrm{kg}}{\mathrm{m}^{3}}$ angenommen werden. Beim Einlassen ändert sich die Dichte, die mit Hilfe der umgestellten allgemeinen Gasgleichung (5.4) zu berechnen ist.

$$
\varrho=\frac{p}{R \cdot T_{\mathrm{R}}}
$$

Die allgemeine Gasgleichung (5.5) wird im Streckenmodell (Abbildung 5.9) auch nach der Integration des Massenstromes zur Berechnung des Aktordrucks verwendet.

$$
p=\frac{m \cdot R \cdot T_{\mathrm{R}}}{V}
$$




\section{Ventilansteuerung}

Bei digitaler Ansteuerung von Ventilen mit Nennspannung hängen Öffnungs- und Schließzeit der Ventile stark von der herrschenden Druckdifferenz ab. Dies wirkt sich gerade bei der Druckregelung in kleinen Volumina negativ aus: Bei zu langen Ansteuerzeiten und großen Druckdifferenzen ergeben sich auch große Drucksprünge. Umgekehrt öffnet das Ventil bei zu geringen Druckdifferenzen und zu kurzen Ansteuerdauern nicht mehr und der Maximaldruck im System wird nicht mehr erreicht.

Eine mögliche Lösung, deren Funktion für größere flexible Fluidaktoren bereits in [190] nachgewiesen wird, ist der Einsatz einer wesentlich aufwändigeren Treiberelektronik. Sie steuert die Ventile während des Öffnens mit erhöhter Spannung an, wodurch die Öffnungszeit und ihre Abhängigkeit von der Druckdifferenz reduziert wird. In diesem Fall wird die Druckregelung als Dreipunktregler ausgeführt, der wiederum einen ausreichend hohen Regelungstakt erfordert.

In diesem Abschnitt wird ein alternativer Ansatz hergeleitet, in dem das druckabhängige Ventilverhalten gezielt ausgenutzt wird. Die Ventile werden dabei mit einem pulsweitenmodulierten (PWM) Signal der Periodendauer $T_{\mathrm{p}}$ angesteuert (Abbildung 5.10). Ein zeitdiskreter Proportionalregler mit der Abtastzeit $T_{\mathrm{A}}$ liefert als Stellgröße die Ansteuerdauer $T_{\mathrm{an}}$, woraus sich das Tastverhältnis $x=\frac{T_{\mathrm{an}}}{T_{\mathrm{p}}}$ ergibt. Bei der Generierung des PWM-Signals wird das Ventilverhalten so berücksichtigt, dass der Regler mit einem niedrigen Regelungstakt betrieben wird.

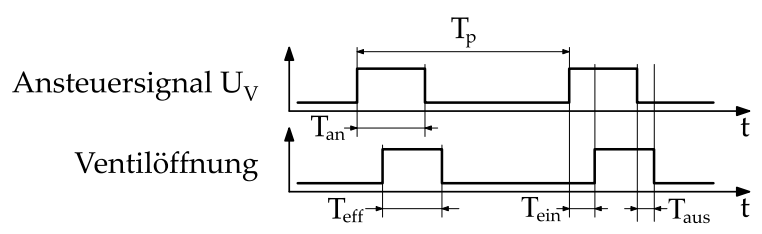

Abbildung 5.10: Zeitverhalten eines mit dem PWM-Signal $U_{\mathrm{V}}$ angesteuerten Ventils.

\section{Ventilmodellierung}

Ventile öffnen und schließen mit Verzögerung gegenüber den Ansteuersignalen (Abbildung 5.10). Es ergibt sich nach Gleichung (5.6) die effektive Öffnungsdauer $T_{\text {eff }}$.

$$
T_{\text {eff }}=T_{\text {an }}-T_{\text {ein }}+T_{\text {aus }}
$$

Werden die Ventile mit einem PWM-Signal der Periodendauer $T_{\mathrm{p}}$ angesteuert, so kann der in Gleichung (5.3) eingeführte Öffnungsgrad $\alpha$ des Ventils auch wie in Gleichung (5.7) als Verhältnis von $T_{\text {eff }} \mathrm{zu} T_{\mathrm{p}}$ beschrieben werden.

$$
\alpha=\frac{T_{\text {eff }}}{T_{\mathrm{p}}}
$$


Zusätzlich wurde beobachtet, dass $T_{\text {eff }}$ von der Druckdifferenz $\Delta p$ am Ventil abhängt. Die Bestimmung von $T_{\text {eff }}\left(\Delta p, T_{\text {an }}\right)$ erfolgt experimentell mit dem Aufbau aus Abbildung 5.8. Der Aktor wird dafür zur Reduzierung von Messfehlern durch ein konstantes Volumen ersetzt. In mehreren Messreihen mit unterschiedlichem $T_{\text {an }}$ wird der Druckverlauf $p(t)$ beim Einlassen und Ablassen aufgenommen. Über die Gleichungen des Streckenmodells wird $T_{\text {eff }}$ aus dem Druck berechnet. So ergeben sich die in Abbildung 5.11 gezeigten Kennfelder $T_{\text {eff }}\left(\Delta p, T_{\text {an }}\right)$. Hervorgehoben sind die Grenzgeraden, bis zu denen das Ventil trotz Ansteuerung nicht öffnet.
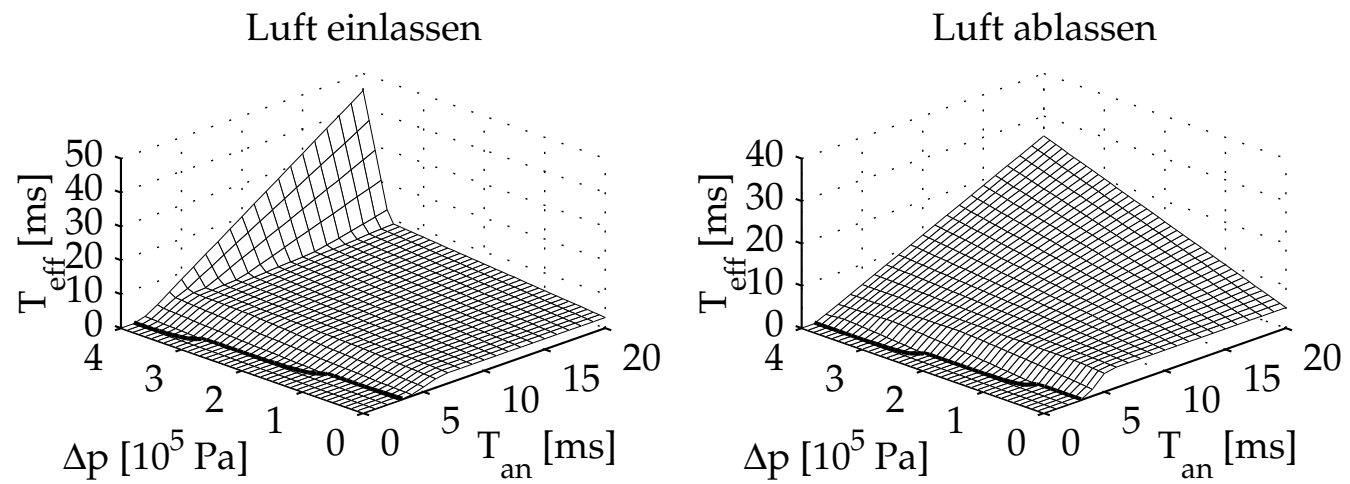

Abbildung 5.11: Kennfelder für $T_{\text {eff }}\left(\Delta p, T_{\text {an }}\right)$ : links Einlassen, rechts Ablassen.

\section{Regleraufbau}

Die Grundstruktur des Druckreglers ist ein zeitdiskreter PI-Regler (Abbildung 5.12) mit der Abtastzeit $T_{\mathrm{A}}$. Er wird durch eine Parameteradaption und eine Führungsgrößenaufschaltung (FGA) ergänzt.

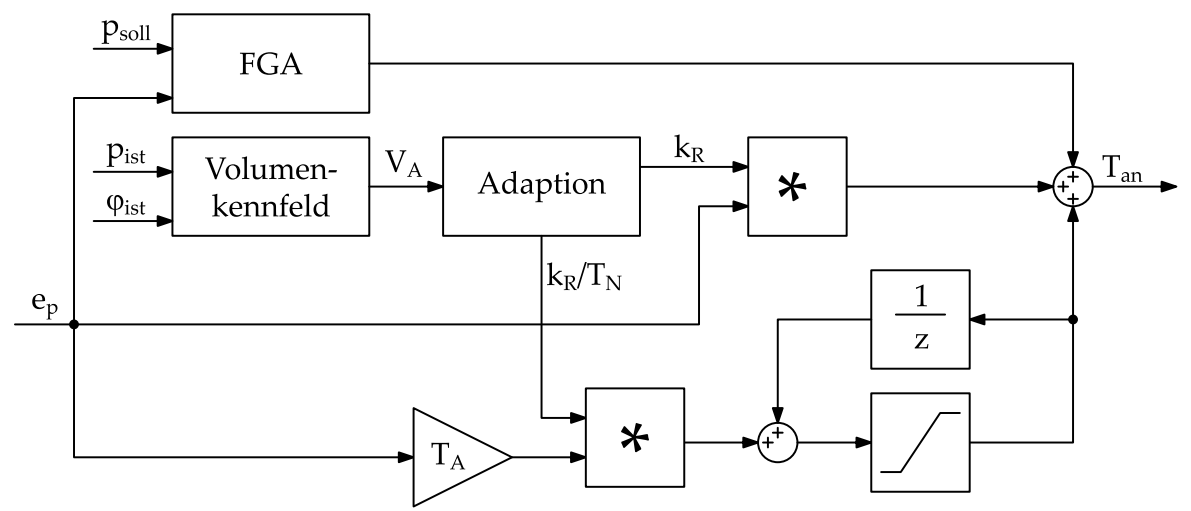

Abbildung 5.12: Struktur des Druckreglers mit Adaption und Führungsgrößenaufschaltung (FGA). 
In die Adaption der Reglerparameter geht das aus Gelenkposition und Aktordruck geschätzte Aktorvolumen ein. Die FGA dient dem schnellen Ansprechen des Reglers bei kleinen Druckfehlern, wie sie z.B. bei einem rampenförmigen Führungsgrößenverlauf auftritt. Sie wählt an Hand des Vorzeichens von $e_{\mathrm{p}}$ die Öffnungskennlinie des Ventils aus und ermittelt aus dem Solldruck an Hand der Grenzgerade die Ansteuerdauer, die gerade noch nicht zum Ansprechen der Ventile ausreicht. Innerhalb einer Totzone wird zur Energieersparnis $T_{\mathrm{an}}=0$ gesetzt.

\subsection{Positionsregelung im hydraulischen Betrieb}

Im hydraulischen Betrieb wird das Medium in beide Richtungen durch eine Zahnradpumpe gefördert [12]. Das Ventil trennt den Aktor bei Erreichen des Sollwerts von der Druckversorgung und dient damit im Gegensatz zum pneumatischen Betrieb nicht primär der Steuerung des Volumenstroms. Die Anordnung der Komponenten ist in Abbildung 5.13 dargestellt.

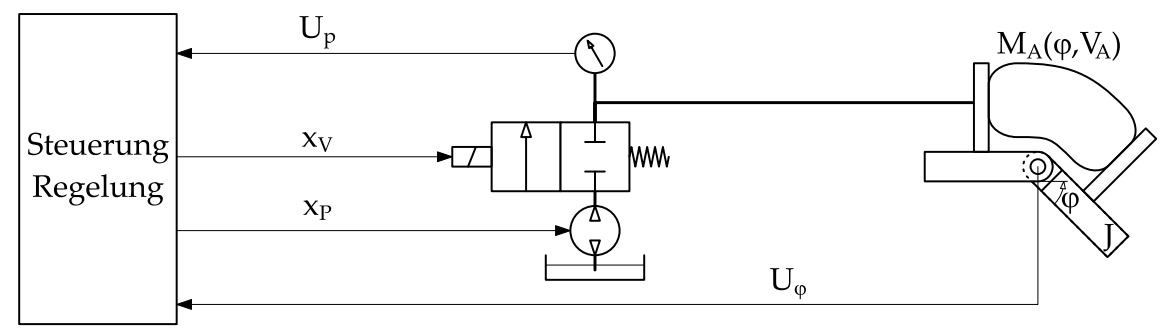

Abbildung 5.13: Schematische Darstellung der hydraulischen Gelenkregelung.

\subsubsection{Streckenmodell}

Zunächst ist das Streckenmodell des Gelenks (Abbildung 5.1) um ein Modell für den Zusammenhang zwischen dem von der Pumpe gelieferten Volumenstrom und dem Druck im Aktor zu erweitern [119]. Bei der Pumpe hängt der Volumenstrom $\dot{V}_{\mathrm{P}}$ sowohl vom Ansteuersignal $x_{P}$ als auch vom Gegendruck $p_{P} \mathrm{ab}$. Der Volumenstrom $\dot{V}_{V}$ des zwischen Pumpe und Aktor geschalteten Ventils wird durch das Ansteuersignal $x_{V}$ und der Druckdifferenz $\Delta p$ zwischen beiden Seiten bestimmt.

In den Verbindungen Pumpe-Ventil und Ventil-Aktor sind die z.B. durch eingeschlossene Luft hervorgerufene Kompressibilität des Mediums sowie die Nachgiebigkeit des Schlauchsystems zu berücksichtigen. Diese werden als hydraulische Kapazitäten $S$ nach Gleichung (5.8) beschrieben.

$$
S=\frac{\dot{V}}{d p / d t}=\frac{d V}{d p}
$$


Die hydraulische Kapazität ist ein Maß dafür, wie schnell der Druck in der als Speicher betrachteten Schlauchleitung in Abhängigkeit vom zufließenden Volumenstrom ansteigt. Der Volumenstrom des Speichers wird über die Knotenregel $\left(\sum \dot{V}_{i}=0\right)$ berechnet.

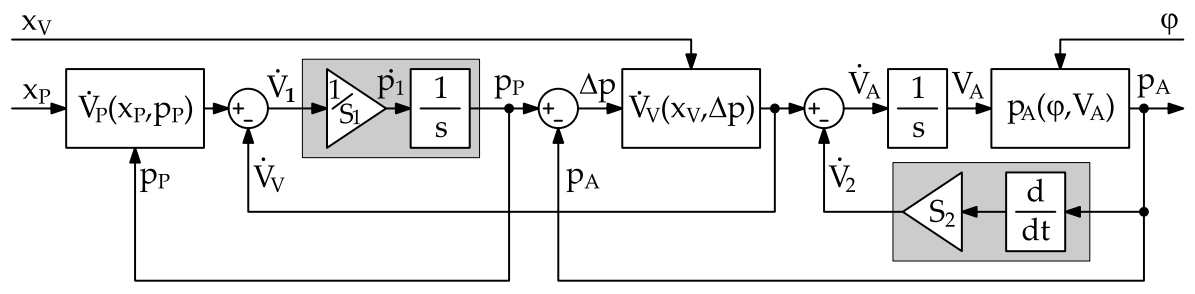

Abbildung 5.14: Modell der hydraulischen Strecke nach [119].

Das hydraulische Streckenmodell ist mit den hervorgehobenen Kapazitäten in Abbildung 5.14 dargestellt. Zunächst ergibt sich aus dem Ansteuersignal $x_{P}$ und dem herrschenden Gegendruck $p_{P}$ der Volumenstrom $\dot{V}_{P}\left(x_{P}, p_{P}\right)$. Der Pumpendruck ergibt sich über die Kapazität $S_{1}$. Nach der Integration des Aktorvolumenstroms $\dot{V}_{A}$ wird über das Volumenkennfeld der Aktordruck $p_{A}\left(\varphi, V_{\mathrm{A}}\right)$ berechnet. Aus seiner Ableitung wird der Volumenstrom $\dot{V}_{2}$ der Kapazität $S_{2}$ zurückgeführt.

\subsubsection{Positionsregler}

Die in Abbildung 5.15 gezeigte Positionsregelung für die beschriebene hydraulische Strecke besteht aus einem zeitdiskreten PI-Regler für die Pumpe sowie einer Totzone zur Ansteuerung des Ventils. Bei Erreichen der Totzone um die Zielposition wird das Ventil geschlossen und der Pumpenregler sowohl eingangs- als auch ausgangsseitig deaktiviert.

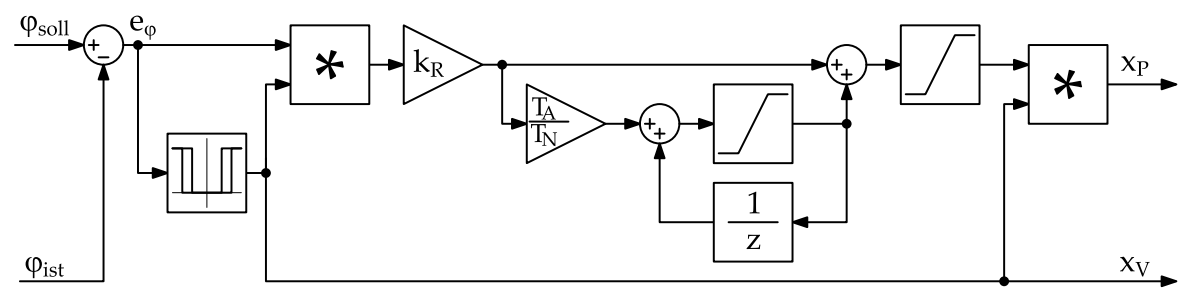

Abbildung 5.15: Zeitdiskreter Regler für die hydraulische Strecke nach [119].

Durch die Begrenzung des I-Anteils und der Stellgröße des Pumpenreglers wird insbesondere beim Öffnen des Gelenks vermieden, dass sich im Rückwärtsbetrieb der Pumpe durch Undichtigkeiten der Luftanteil im System erhöht. 


\subsection{Momentensteuerung}

Bei der bisherigen Diskussion der Positionsregelung von Gelenken wurde die für das Greifen entscheidende Interaktion mit Objekten kaum berücksichtigt. Bisher beantwortet der Regler jeden durch einen Objektkontakt hervorgerufenen bleibenden Positionsfehler mit einem bis an die Stellbegrenzung ansteigenden Aktordruck. Damit steigt auch das ausgeübte Gelenkmoment und die auf das Objekt ausgeübte Kraft. Für ein kontrolliertes Zugreifen ist dieses Verhalten nicht erwünscht. Es kann jedoch durch eine Erweiterung des Reglers vorteilhaft ausgenutzt werden.

\subsubsection{Testumgebung}

Zur Erprobung verschiedener Lösungsansätze werden der bei der pneumatischen Positionsregelung verwendete Eingelenkfinger und ein modifizierter Aktorteststand (Abbildung 5.16) eingesetzt. Während der Finger proximal wie bei der Vermessung von Aktoren und Winkelsensoren fest eingespannt ist, kann das distale Fingerglied durch den Aktor bewegt werden. Die zweite Fingereinspannung wird durch einen verstellbaren Halter an der Positioniereinheit mit Schrittmotor und Momentensensor ersetzt. So können Objekte relativ zum Finger genau wiederholbar positioniert werden.

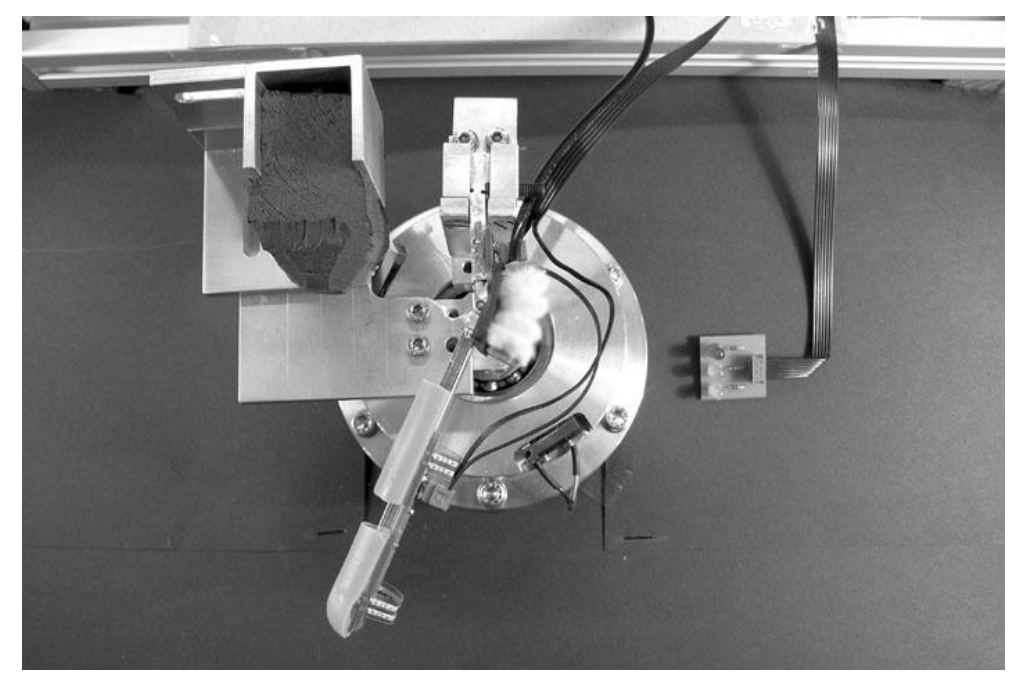

Abbildung 5.16: Testaufbau für die Momentensteuerung des pneumatischen Positionsreglers.

Die Anbringung der Gelenkachse des Fingers in der Flucht der Drehachse des Schrittmotors ermöglicht die Übertragung identischer Kontaktszenarien auf verschiedene Gelenkstellungen. Dabei ist die geometrische Kontaktposition über die Schrittmotorposition genau bekannt. Der Momentensensor in der Positioniereinheit ermöglicht zudem den Vergleich mit dem aus den Kontaktkräften $F_{1}, F_{2}$ der FSR-Sensoren und den Längen der Hebelarme $l_{1}=40 \mathrm{~mm}, l_{2}=80 \mathrm{~mm}$ berechne- 
ten Kontaktmoments $M_{\mathrm{FSR}}$. Gleiches gilt für das aus Aktordruck und Gelenkwinkel geschätzten Aktormoment $M_{\mathrm{A}}(\varphi, p)$.

\subsubsection{Statische Momentenbegrenzung}

Eine in der vorhandenen Reglerstruktur realisierbare Methode ist die modellbasierte Begrenzung des Aktormoments über die Stellgröße des Positionsreglers. [12]. Das Aktorverhalten $M_{\mathrm{A}}(\varphi, p)$ wird hierzu in $p\left(\varphi, M_{\mathrm{A}}\right)$ umgerechnet. So lässt sich zur aktuellen Winkelposition $\varphi_{\text {ist }}$ aus einem gegebenen Maximalmoment $M_{\max }$ der für den Aktor zulässige Maximaldruck $p_{\max }\left(\varphi_{\text {ist }}, M_{\max }\right)$ bestimmen (Abbildung 5.17).

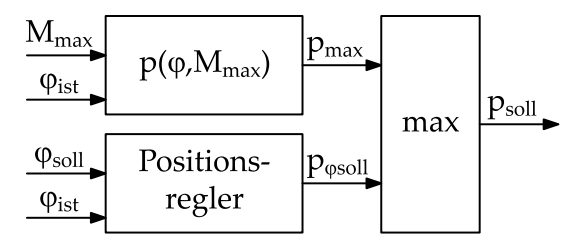

Abbildung 5.17: Prinzip der Momentenbegrenzung des pneumatischen Positionsreglers.

Durch die Momentenbegrenzung ist z.B. ein vorsichtiges Greifen eines Objekts möglich. Voraussetzung ist dabei, dass die vorgegebene Sollwinkelposition größer ist als die bei Objektkontakt erreichte Gelenkposition. Die Planung muss also von einem etwas zu kleinen Objekt ausgehen und dementsprechend größere Gelenkwinkel vorgeben.

Abbildung 5.18 oben zeigt das Verhalten eines auf diese Weise begrenzten Positionsreglers. Im Experiment trifft der Finger mit einer Sollposition von $70^{\circ}$ wiederholt bei $50^{\circ}$ auf ein Objekt aus einem festen Schaum. Das zulässige Maximalmoment wird in jedem Zyklus von 0,15 $\mathrm{Nm}$ über 0,1 Nm;0,05 $\mathrm{Nm} ; 0,025 \mathrm{Nm}$ auf schließlich 0,01 Nm reduziert (Abbildung 5.18, Mitte).

$\mathrm{Zu}$ Anfang erreicht der Regler die Momentenbegrenzung trotz maximal möglichem Druck nicht, da der Aktor in dieser Stellung das erlaubte Moment gar nicht aufbringen kann. In den Zyklen 2, 3 und 4 erreicht der Regler das kleiner werdende Maximalmoment, wodurch auch die geringer werdende Deformation des Schaumstoffs begründet ist. Der letzte Zyklus endet wegen des zu geringen $M_{\max }$ von 0,01 Nm ohne Objektkontakt. Die Bewegung des Gelenks wird direkt zu Beginn der Führungsgrößenrampe gestoppt, da durch Approximationsfehler im Aktorkennfeld ein Maximaldruck berechnet wird, der für die Bewegung des Gelenks zu nicht ausreichend ist. In den gezeigten Fällen ist zu erkennen, dass kein direkter Eingriff in die Aktivität des Reglers stattfindet: beim Strecken des Fingers beginnt die Bewegung des Gelenks erst verzögert, da der I-Anteil des Reglers erst abgebaut werden muss.

Die dritte Grafik in Abbildung 5.18 stellt die Momentenschätzfehler $\Delta M_{\mathrm{FSR}}=$ $M_{\mathrm{FSR}}-M_{\text {ist }}$ und $\Delta M(\varphi, p)=M(\varphi, p)-M_{\text {ist }}$ dar. Bei der Momentenschätzung aus dem Signal des Kraftsensors ist zu ersehen, dass jeweils zu Beginn und Ende 

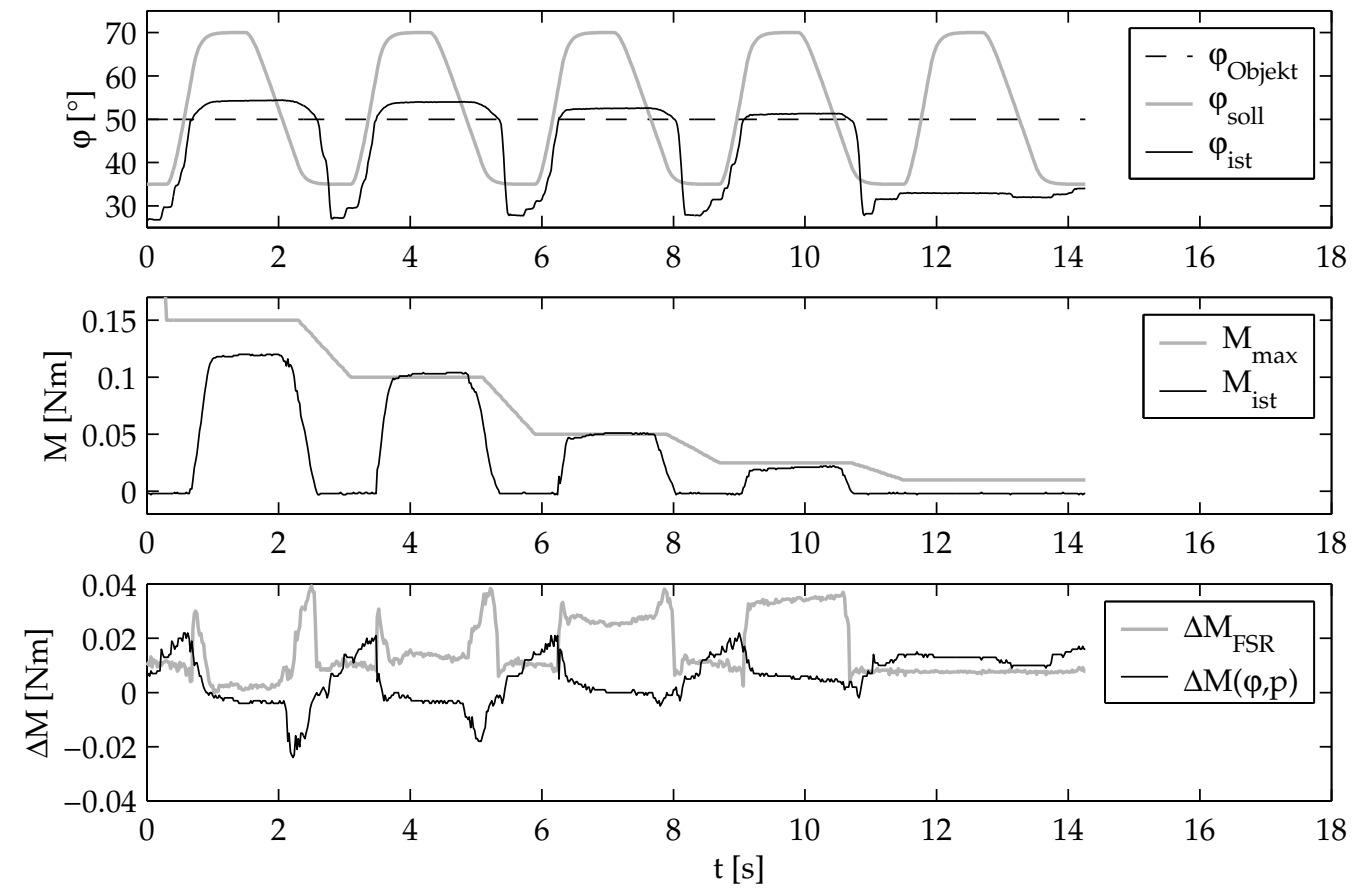

Abbildung 5.18: Messung: Positionsregler mit statischer Momentenbegrenzung. Der Finger fährt mit $\varphi_{\text {soll }}=70^{\circ}$ wiederholt gegen ein Objekt bei $\varphi_{\text {Objekt }}=50^{\circ}$.

der Kontaktphase ein überhöhtes Moment gemessen wird. Dagegen wird bei der Momentenschätzung aus Gelenkwinkel und Druck nur zu Beginn der Schließbewegung ein zu großes Moment ermittelt. Wird das Gelenk geöffnet, so wird ein zu kleines Moment geschätzt. Tabelle 5.1 führt die zeitlich gemittelten Fehler der Momentenschätzung auf. Obwohl das betrachtete Szenario auf Grund der genauen Positionierung des Objekts als vorteilhaft für den FSR-Sensor eingeschätzt werden kann, liefert er einen etwa doppelt so großen Fehler wie $M(\varphi, p)$.

\begin{tabular}{l|c|c|} 
Abweichung & $\begin{array}{c}\text { Fehlerbetrag } \\
{[m N m]}\end{array}$ & $\begin{array}{c}\text { rel. Fehlerbetrag } \\
{[\%]}\end{array}$ \\
\hline$\Delta M(\varphi, p)$ & 8,2 & 1,52 \\
$\Delta M_{\mathrm{FSR}}$ & 15,3 & 2,82 \\
\hline
\end{tabular}

Tabelle 5.1: Mittlerer absoluter und relativer Momentenschätzfehler bezogen auf $M_{\max }=0,54 \mathrm{Nm}$ über Aktormodell und FSR-Sensoren bei der statischen Momentenbegrenzung.

Die statische Momentenbegrenzung ist damit vor allem zur Begrenzung großer Momente geeignet, wodurch z.B. Schäden an zu greifenden Objekten vermieden werden können. Für die Begrenzung auf sehr kleine $M_{\max }$ bei gleichzeitig 
geforderter Bewegung ist das Verfahren nicht geeignet.

\subsubsection{Dynamische Momentenbegrenzung}

Soll ein kleines statisches Maximalmoment zusammen mit einer unveränderten Bewegungsdynamik realisiert werden, kann dies mit Hilfe des zu Beginn des Kapitels hergeleiteten Streckenmodells (Gleichung (5.1), Abbildung 5.1) erreicht werden. Es erlaubt die Berechnung des Mindestmoments für die durch die geforderte Bewegung aus der ersten und zweiten Ableitung der Führungsgröße. Damit kann

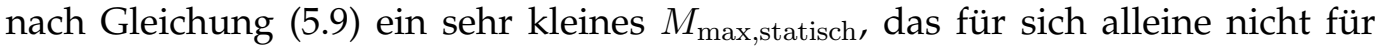
eine Bewegung ausreicht, während einer Bewegung so weit angehoben werden, dass die vorgegebene Dynamik erreicht wird.

$$
M_{\text {max }, \text { dynamisch }}=M_{\text {max,statisch }}+J \cdot \ddot{\varphi}_{\text {soll }}+d \cdot \dot{\varphi}_{\text {soll }}
$$

Der Einsatz dieses Verfahrens wird in Abbildung 5.19 demonstriert. Das Fingergelenk wird dabei mit den vier Objektpositionen $40^{\circ} ; 50^{\circ} ; 60^{\circ}$ und $70^{\circ}$ von $30^{\circ}$ nach $70^{\circ}$ bewegt. Als Objekt wird ein weicher Schaumstoffanschlag mit einer Federkonstante von $0,2 \frac{\mathrm{N}}{\mathrm{mm}}$, also $\frac{0,006 \mathrm{Nm}}{1^{\circ}}$ bei einem Bewegungsradius von $40 \mathrm{~mm}$ verwendet.
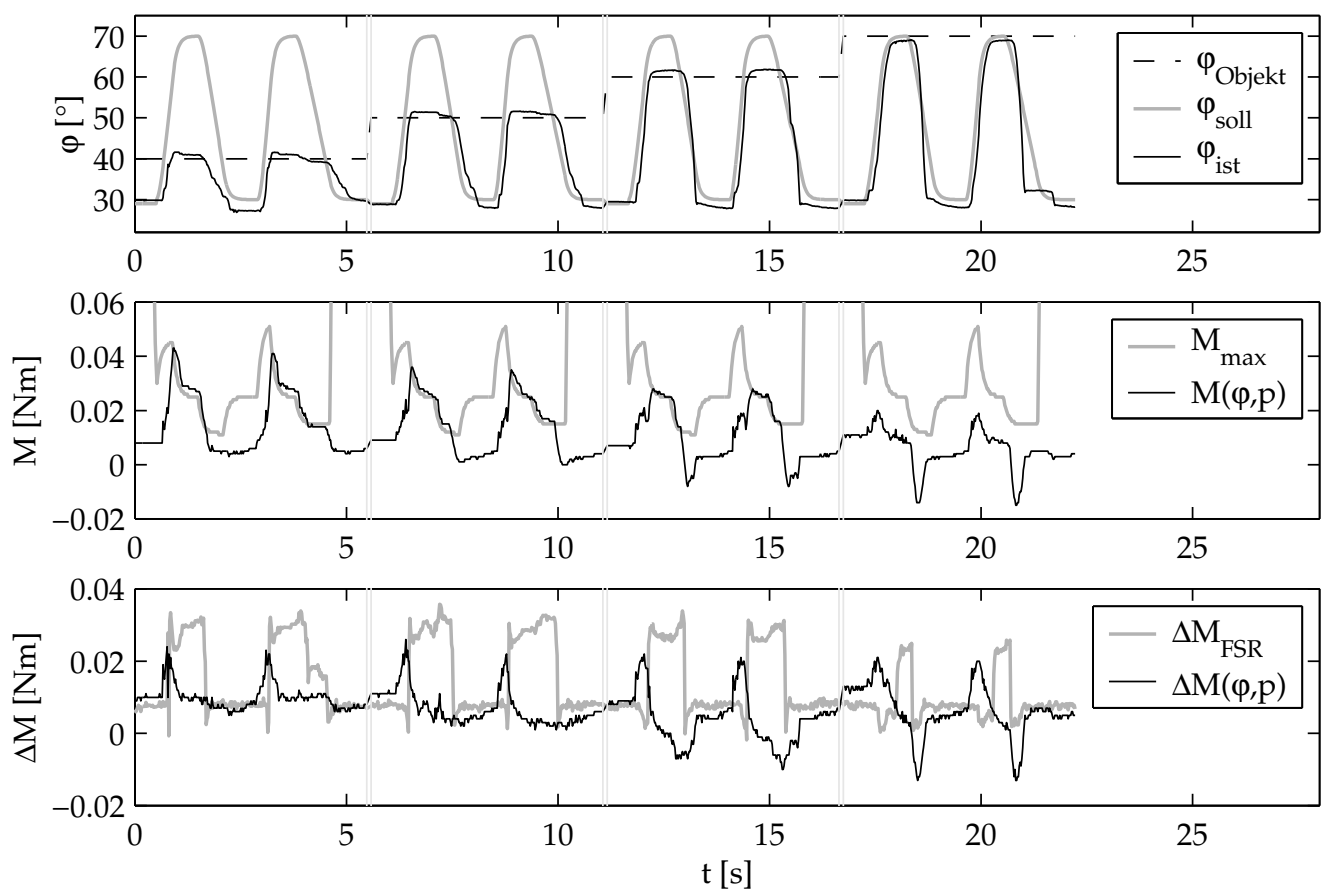

Abbildung 5.19: Messung: Positionsregler mit dynamischer Momentenbegrenzung. Der Finger fährt mit $\varphi_{\text {soll }}=70^{\circ}$ wiederholt gegen ein Objekt an den Positionen $\varphi_{\text {Objekt }}=40^{\circ} ; 50^{\circ} ; 60^{\circ}$ und $70^{\circ}$.

So wird mit einem gegenüber dem Stillstand verursachenden $M_{\max , \text { statisch }}=$ $0,01 \mathrm{Nm}$ mit einem $M_{\text {max,statisch }}=0,015 \mathrm{Nm}$ und der dynamischen Momentenbe- 
grenzung in drei der vier Fälle das weiche Objekt ohne deutliche Verformungen erreicht. Ein leichter Rückgang der Gelenkposition ist gerade bei den Kontaktpositionen $40^{\circ}$ und $50^{\circ}$ nach dem Ende der Schließbewegung erkennen. Er wird durch den nach dem Ende der Sollbewegung kleiner werdenden Wert von $M_{\max , \text { dynamisch }}$ verursacht (Abbildung 5.19, Mitte). Dort ist auch zu erkennen, dass die Momentenbegrenzung bereits während der Sollbewegung aktiv wird.

Tabelle 5.2 führt die zeitlich gemittlelten Fehler der Momentenschätzung aus Abbildung 5.19, unten auf. Trotz des veränderten Szenarios mit geringeren Kontaktkräften entsprechen die Werte weitgehend denen des vorangegangenen $\mathrm{Ab}$ schnitts.

\begin{tabular}{l|c|c|} 
Abweichung & $\begin{array}{c}\text { Fehlerbetrag } \\
{[m N m]}\end{array}$ & $\begin{array}{c}\text { rel. Fehlerbetrag } \\
{[\%]}\end{array}$ \\
\hline$\Delta M(\varphi, p)$ & 7,8 & 1,45 \\
$\Delta M_{\mathrm{FSR}}$ & 13,5 & 2,50 \\
\hline
\end{tabular}

Tabelle 5.2: Mittlerer absoluter und relativer Momentenschätzfehler bezogen auf $M_{\max }=0,54 \mathrm{Nm}$ über Aktormodell und FSR-Sensoren bei der dynamischen Momentenbegrenzung.

\subsubsection{Steifigkeitssteuerung}

Das im vorangegangenen Abschnitt beschriebene Verfahren wirkt nur während einer Bewegung des Gelenks und nicht während Phasen einer unveränderten Führungsgröße. Für das Greifen ohne Gelenkbewegung kann die Momentenbegrenzung auf eine andere Weise sinnvoll angepasst werden.

$\mathrm{Zu}$ Grunde liegt die Überlegung, dass das ausgeübte Maximalmoment durch den Positionsfehler beeinflusst wird: Bei geringen Positionsfehlern soll das Gelenk mit einem geringen Maximalmoment bewegt werden, dass ein auftretender $\mathrm{Ob}$ jektkontakt von der Positionsregelung nicht mit schnell ansteigenden Kontaktmoment beantwortet wird. Tritt dagegen sukzessive ein großer Positionsfehler auf, so ist auch ein größeres Moment zulässig, mit dem das Objekt auf seine Zielposition bewegt werden kann. Dieses Verhalten lässt sich nach Gleichung (5.10) durch Ergänzung des statischen Maximalmoments $M_{\text {max,statisch }}$ um einen winkelfehlerabhängigen Anteil mit der Federkonstante $c_{R}$ erreichen.

$$
M_{\text {max }, F e d e r}(\varphi)=M_{\text {max,statisch }}+c_{R} \cdot\left(\varphi_{\text {soll }}-\varphi_{\text {ist }}\right)
$$

Ähnliche Überlegungen liegen z.B. auch der bei einigen Händen ohne nachgiebige Antriebe eingesetzten kartesischen Steifigkeitsregelung zu Grunde, wobei die Momente anstatt aus den Winkelfehlern der Gelenke aus den kartesischen Positionsfehlern der Fingerspitzen ermittelt werden. So übt beim Greifen immer der Finger mit der größten Positionsabweichung auch das größte Moment auf das Objekt aus. Zu berücksichtigen ist dabei, dass für dieses Verfahren sehr schnell und präzise auf Veränderungen der Kontaktkraft reagierende Sensoren, Regler und Antriebe notwendig sind. 
Bei elastischen Aktoren dient Gleichung (5.10) dem Erhalt und der gezielten Beeinflussung des in Kapitel 3 hergeleiteten Nachgiebigkeitsverhaltens. Für die Dynamik des Gelenks ist ebenfalls vorteilhaft, dass das Maximalmoment bei einem schnell ansteigenden Positionsfehler, wie er z.B. nach einem Sprung der Führungsgröße auftritt, angehoben wird und die geforderte Gelenkdynamik auch ermöglicht.
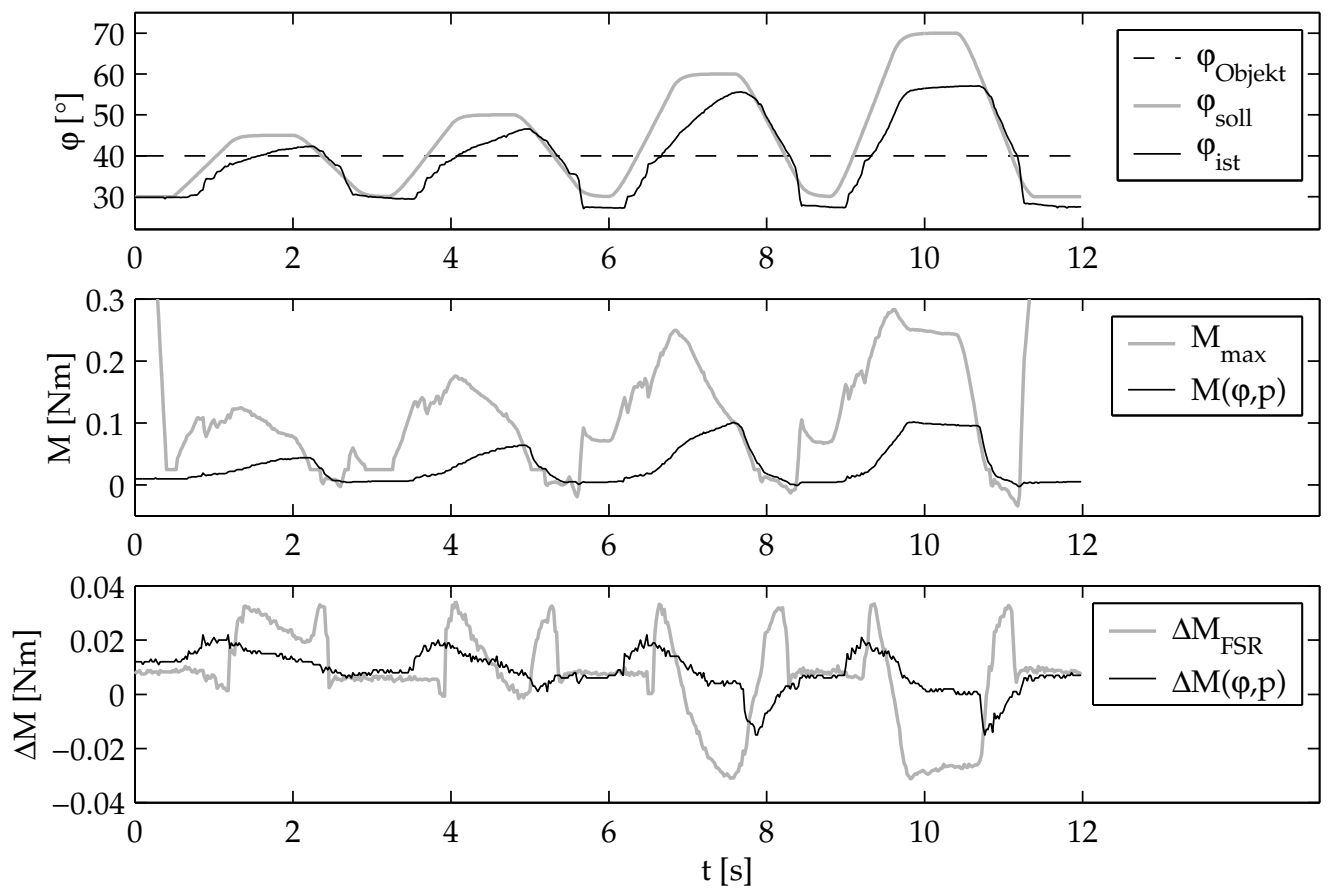

Abbildung 5.20: Messung: Positionsregler mit steifigkeitsabhängiger Momentenbegrenzung.

Abbildung 5.20 zeigt Messergebnisse mit diesem Verfahren. Der Finger fährt mit $c_{R}=\frac{1 \mathrm{Nm}}{60^{\circ}}$ dreimal hintereinander mit den Zielpositionen $45^{\circ} ; 50^{\circ} ; 60^{\circ}$ und $70^{\circ}$ gegen ein Objekt aus Schaumstoff an der Position $40^{\circ}$. Erwartungsgemäß steigt die Deformation des Objekts mit der vorgegebenen Sollposition, wobei sich in der Kontaktphase die Gelenkbewegung verlangsamt. Die Winkelgeschwindigkeit ergibt sich hier über den I-Anteil des Positionsreglers, den dadurch ansteigenden Aktordruck und das sich daraus ergebende Aktormoment, dem wiederum das Kontaktmoment entgegenwirkt. Während der letzten Bewegung wird bei $t=9,8 \mathrm{~s}$ der maximale Aktordruck von $6 \cdot 10^{5} \mathrm{~Pa}$ erreicht (Abbildung 5.20, Mitte). Ab diesem Punkt kann das Aktormoment nicht weiter ansteigen.

Auch in diesem Testszenario zeigen sich für die Leistungsfähigkeit der Fingersensoren (Abbildung 5.20, unten) ähnliche Werte wie in den vorangegangenen Versuchen (Tabelle 5.2). Bei großen Kontaktmomenten wird im dritten und vierten Zyklus mit den Kraftsensoren ein zu geringes Moment ermittelt, da wegen der starken Deformation des Schaumstoffobjekts nicht mehr die gesamte Kontaktkraft 
auf den Kraftsensor eingeleitet wird.

\begin{tabular}{l|c|c|} 
Abweichung & $\begin{array}{c}\text { Fehlerbetrag } \\
{[m N m]}\end{array}$ & $\begin{array}{c}\text { rel. Fehlerbetrag } \\
{[\%]}\end{array}$ \\
\hline$\Delta M(\varphi, p)$ & 9,7 & 1,81 \\
$\Delta M_{\mathrm{FSR}}=F_{1} \cdot l_{1}+F_{2} \cdot l_{2}$ & 14,6 & 2,70 \\
\hline
\end{tabular}

Tabelle 5.3: Mittlerer absoluter und relativer Momentenschätzfehler bezogen auf $M_{\max }=0,54 \mathrm{Nm}$ über Aktormodell und FSR-Sensoren bei der positionsabhängigen Momentenbegrenzung.

\subsubsection{Kontakterkennung}

An dieser Stelle sei auch noch auf einen entscheidungstheoretischen Ansatz zur Erkennung eines Objektkontakts über das Momentenmodell des Aktors hingewiesen. Die Information des Objektkontakts kann z.B. für das Umschalten zwischen einer Positions- und einer Momentenregelung verwendet oder zum Stoppen einer Gelenkbewegung beim unerwarteten Berühren eines Objekts verwendet werden.

Das in [14] beschriebene Verfahren berücksichtigt nicht nur den beim Greifen selten zu erwartenden statischen Fall, in dem ein bewegtes Objekt auf einen stehenden Finger trifft, sondern auch die Annäherung mit dem Finger an ein stehendes Objekt. In diesem dynamischen Fall ist das auf das Objekt ausgeübte Moment wegen der Dämpfung des Gelenks und der Massenträgheit des Fingers geringer als das geschätzte Gelenkmoment.

\subsection{Finger- und Gelenkkoordination}

In den bisherigen Abschnitten dieses Kapitels wurden nur einzelne Gelenke betrachtet. Ist dagegen eine Hand mit mehreren flexiblen Fluidaktoren und unterschiedlichen Führungsgrößen zu steuern oder zu regeln, so sind die mechanischen Abhängigkeiten zwischen den Aktoren beim Entwurf des Steuerungs- und Regelungssystems zu berücksichtigen. In den folgenden beiden Abschnitten wird ein Lösungsansatz für den hydraulischen Betrieb erörtert und anschließend auf den pneumatischen Fall übertragen sowie betrachtet, an welchen Stellen das Verhalten der Finger durch eine geeignete Sensorausstattung verbessert werden kann.

\subsubsection{Hydraulischer Betrieb}

Die Vervielfachung der Anordnung aus Abbildung 5.13 ergibt eine Hand mit einer Pumpe je Aktor, womit eine kompakte Leichtbauhand nicht mehr realisierbar ist. Wird die gesamte Hand stattdessen wie in Abbildung 5.21 von einer einzigen Pumpe versorgt, führt dies zu einer Kopplung der Aktoren. Gleichgerichtete Bewegungen unterschiedlicher Geschwindigkeit sind nur über Ventilansteuerung im laufenden Pumpenbetrieb realisierbar. Ohne die Messung des Aktordrucks sind gegenläufige Gelenkbewegungen nicht zuverlässig realisierbar. 


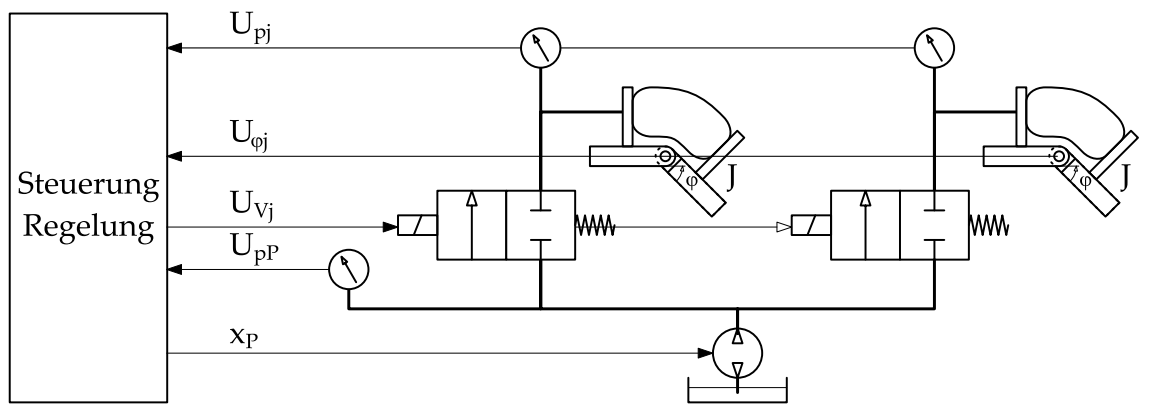

Abbildung 5.21: Hydraulische Regelung mehrerer Aktoren.

Ein möglicher Ansatz für den Betrieb einer mehrgelenkigen Hand ist das gruppenweise sequentielle Abarbeiten von Vorwärts- und Rückwärtsbewegungen [110]. Hierzu werden die Einzelfehler $e_{\varphi j}$ aller $j$ Gelenke in die zwei Gruppen positiver $\left(E_{\text {pos }}\right)$ und negativer $\left(E_{\text {neg }}\right)$ Fehler aufsummiert. Ausgeschlossen sind alle Gelenke, deren Einzelfehler sich innerhalb einer Totzone befindlichen. Über einen Zustandsautomaten (Abbildung 5.22) wird entschieden, welche der beiden Gruppen durch die Pumpe bedient wird und welche Ventile zu öffnen sind. Die Regelung der Pumpe erfolgt wie beim hydraulisch betriebenen Einzelgelenk über einen PI-Regler. Liegen alle Einzelfehler innerhalb der Totzone, so wird auch die Pumpe abgeschaltet. Die Richtungsumkehr erfolgt erst, wenn die Fehlersumme der Gegenrichtung deutlich größer sind als die aktuell bearbeiteten Fehler. Hierzu wird der Gewichtungsfaktor $r_{\mathrm{P}}>1$ verwendet.

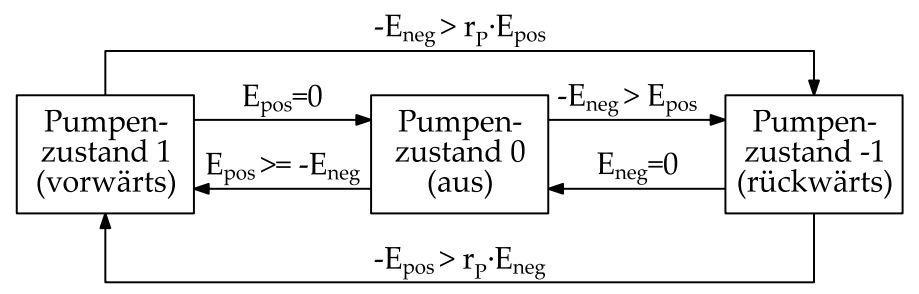

Abbildung 5.22: Zustandsautomat für hydraulische Pumpenregelung.

Der Nachteil dieser Methode ist, dass das Öffnen des Ventils z.B. unmittelbar nach der Richtungsumkehr der Pumpe oder bei durch Objektkontakt gestiegenem Aktordruck zu einem unerwünschten Ansteigen des Fehlers führt. Das Verfahren kann durch den Einsatz von Drucksensoren verbessert werden, indem zusätzlich zum Positionsfehlers auch die Differenz zwischen dem gemeinsamen Pumpendruck und dem Druck des einzelnen Aktors herangezogen wird. Das Ventil wird nur dann geöffnet, wenn Druckdifferenz und Positionsdifferenz das gleiche Vorzeichen haben.

Diese Vorgehensweise ermöglicht zumindest in manchen Situationen gegenläufige Bewegungen. Beispiel ist das Anfahren ähnlicher mittlerer Gelenkpositio- 
nen, mit wenn sich manche Gelenke im stark gebeugten Zustand und andere im gestreckten Zustand befinden. Mit Drucksensoren können beide Anforderungen zeitgleich bearbeitet werden, während dies ohne die Druckinformationen aus den einzelnen Aktoren nicht zuverlässig möglich ist.

\subsubsection{Pneumatischer Betrieb}

Die Vervielfachung der pneumatischen Regelung aus Abbildung 5.8 schafft zunächst keine Abhängigkeiten für die Bewegung mehrerer Gelenke, es werden jedoch zwei Ventile je Aktor benötigt. In Analogie zur hydraulischen Verschaltung kann die Druckversorgung über ein 3/2-Wege-Ventil realisiert werden, das zwischen dem Versorgungsdruck $p_{V}$ des Kompressors und dem Umgebungsdruck $p_{0}$ umschaltet (Abbildung 5.23).

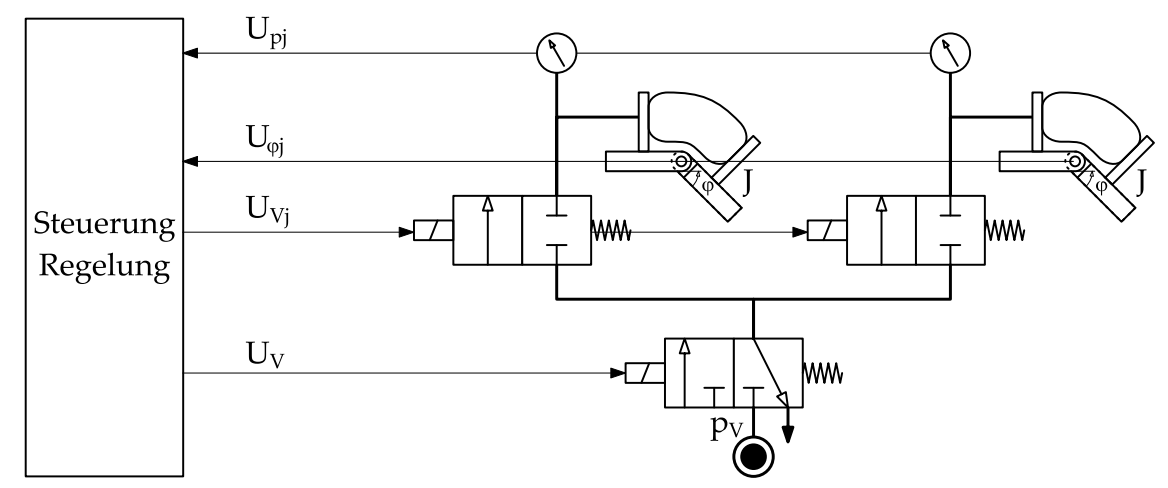

Abbildung 5.23: Alternative Schaltung der pneumatischen Gelenkregelung mehrerer Aktoren mit reduzierter Ventilanzahl.

Wird in dieser Schaltung der Ansatz der Positionsregelung mit einer unterlagerten Druckregelung weiter verfolgt, so ist die Ausstattung der einzelnen Aktoren mit Drucksensoren unerlässlich.

\subsubsection{Fingerpriorisierung}

Je nach Greifaufgabe sind den verschiedenen Fingern unterschiedliche Prioritäten zuzuordnen. So ist z.B. bei einem Pinzettengriff, mit dem ein kleines Objekt zwischen den Spitzen von Daumen und Zeigefinger gegriffen werden soll, das präzise Aufeinandertreffen der Fingerspitzen von höchster Bedeutung. Hierfür sind bei einer anthropomorphen Hand ohne Freiheitsgrade zur Abduktion der Finger die beiden proximalen Gelenke des Daumens verantwortlich. Tritt an dieser Stelle ein Positionsfehler auf, kann das sichere Greifen nicht mehr gewährleistet werden.

Zur Vermeidung solcher Probleme ist der Zustandsautomat aus Abbildung 5.22 um eine gewichtete Summenbildung der positiven und negativen Fehler zu erweitern. So können die für einen Greifvorgang besonders wichtigen Finger oder Einzelgelenke mit einer höheren Priorität versehen werden. Umgekehrt können aber auch Finger mit einer besonders geringen Priorität bewegt werden, im 
obigen Beispiel mit dem Pinzettengriff sind dies der kleine Finger und der Ringfinger. Eine weitere Verbesserung kann die Anpassung der Totzonengröße sein.

Solche Ansätze spiegeln sich bei der Robonaut Hand der NASA [120] sogar im mechanischen Design der Hand wieder: Daumen, Zeige- und Ringfinger sind für die Manipulation von Objekten mit mehreren unabhängigen Freiheitsgraden ausgestattet. Ringfinger und kleiner Finger sind nur mit je zwei Gelenken zur Flexion und einem Antrieb ausgestattet und übernehmen nur Hilfsfunktionen bei Kraftgriffen.

\subsection{Steuerungsstruktur für Hände und Arme}

Im bisherigen Teil dieses Kapitels wurde die Regelung und Steuerung von Gelenken losgelöst von übergeordneten Systemen betrachtet. Aus dem Kontext eines humanoiden Roboters mit einer eigenen Steuerung und anthropomorphen Armen ergeben sich weitere Anforderungen für die Fähigkeiten und Schnittstellen einer Greifsteuerung [125, 146].

Dieser Abschnitt gibt eine Übersicht zur Gesamtstruktur eines solchen Systems, in den folgenden Abschnitten wird auf die Schwerpunkte der Greifplanung, der Handsteuerung und der Hand- Arm- Koordination weiter eingegangen.

Die gewählte Systemstruktur (Abbildung 5.24) entkoppelt Hand und Arm bei der Planung und Durchführung von Bewegungen und Greifvorgängen weitgehend. So steht bei der Betrachtung des Arms die Positionierung der Hand als Ganzes im Vordergrund; ihre einzelnen Freiheitsgrade werden nicht betrachtet. Umgekehrt soll die Handsteuerung möglichst wenig globale Informationen benötigen. Eine übergeordnete Robotersteuerung, die auch weitere Aufgaben des humanoiden Roboters übernimmt, liefert die Aufgabeninformationen und dient dem Informationsaustausch zwischen Arm und Hand.

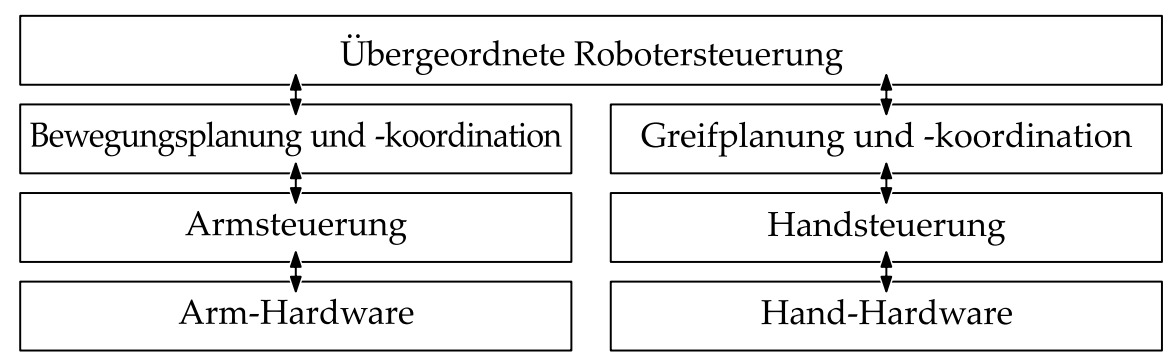

Abbildung 5.24: Struktur für die Steuerung von Armen und Händen eines humanoiden Roboters.

Als Trennungspunkt von Arm und Hand wird der TCP (tool center point) des Arms gewählt. Er liegt im Arm distal vom Handgelenk und ist gleichzeitig der Ursprung des Koordinatensystems der Hand. 


\subsection{Greifplanung und -koordination}

Die Greifplanung und -koordination ist die Schnittstelle zwischen der übergeordneten Robotersteuerung und der Handsteuerung (Abbildung 5.25). In der Planungsphase ist ihre Aufgabe die Ableitung der Handaktionen aus einer gestellten Greifaufgabe. Dabei bestimmt sie auch Randbedingungen für die Bewegungsplanung des Armes. Während der Ausführung der geplanten Aufgabe übernimmt sie die Bewegungssynchronisation von Arm und Hand.

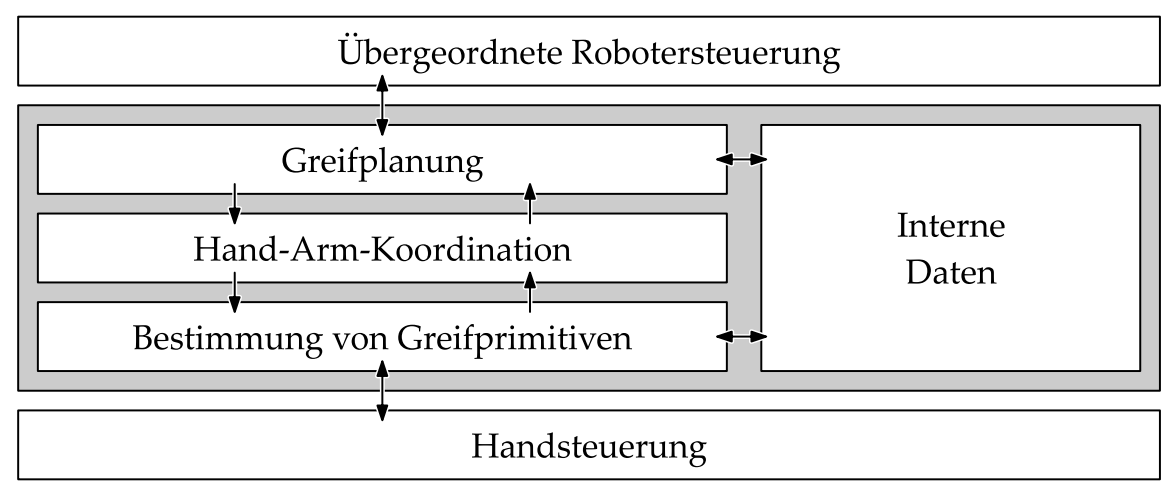

Abbildung 5.25: Hierarchie der Greifplanung und -koordination.

\subsubsection{Modellbasierte Greifplanung}

Die in der Robotik etablierte modellbasierte Greifplanung beinhaltet die Ermittlung geeigneter Kontaktpunkte für die Fingerspitzen auf der Objektoberfläche, was ein sehr genaues Objektmodell voraussetzt. Gleichzeitig müssen Annahmen über das Reibungsverhalten an den Kontaktpunkten getroffen werden [156]. Dabei sind gerade die für das sichere Greifen als vorteilhaft angesehenen flächigen Kontakte mit elastischen Fingerspitzen besonders schwierig zu modellieren. Die kinematischen Eigenschaften der Hand sind als zusätzliche Randbedingungen zu berücksichtigen. Die Frage der Menschenähnlichkeit der Bewegungen stellt sich dabei nicht, da die meisten hierbei verwendeten Hände ohnehin keine anthropomorphe Gestalt besitzen.

\subsubsection{Greifplanung für einen humanoiden Roboter}

Der Greifplanung kommt durch die für einen humanoiden Roboter geforderte Menschenähnlichkeit der Bewegungen eine neue Aufgabe zu: sie hat nun unter Berücksichtigung von Greifaufgabe und Objektgeometrie aus einem Repertoire menschentypischer Greifmuster das am besten geeignete auszuwählen und gegebenenfalls mit Parametern wie z.B. der Objektgröße zu versehen. Dieses Verfahren kommt zudem Händen mit kinematischen Restriktionen wie z.B. einer fehlenden oder eingeschränkten Abduktion der Finger entgegen. Position und Ausrichtung 
der Hand im Raum sind zu bestimmen und in Interaktion mit der Bewegungsplanung des Armes auf Realisierbarkeit zu überprüfen. Die Handsteuerung erhält von der Greifplanung nur relativ grobe Vorgaben zum auszuführenden Greifvorgang. Abweichungen von der Referenzform eines Objekts während des Greifens sind somit von der Handsteuerung selbsttätig mit Hilfe der Sensordaten ausgleichen.

\subsubsection{Generierung von Greifmusterdaten}

Die später als Führungsgrößen für die Regelung der Hand verwendeten Daten von Greifmustern lassen sich durch Aufzeichnung von menschlichen Beispielbewegungen erzeugen. Als Messeinrichtung wird ein Datenhandschuh verwendet, der zusätzlich mit taktilen Sensoren ausgestattet ist. Dieses Verfahren wird bereits erfolgreich für das Programmieren durch Vormachen genutzt [204]. Dabei erleichtern die taktilen Sensoren die Erkennung der Greifmuster [57] und die Segmentierung der vorgeführten Bewegung in die einzelnen Greifphasen [205]. Verwendet werden diese Informationen dort, um den demonstrierten Greifvorgang so weit zu abstrahieren, dass er sich auf andere Greifergeometrien übertragen lässt. In gleicher Weise sind die in Abschnitt 4.2 .8 beschriebenen taktilen Sensoren zur Greifkraftmessung einsetzbar.

$\mathrm{Zu}$ messen sind für alle $j$ Gelenke die Gelenkwinkel $\varphi_{j}(t)$ und die Kontaktkräfte $F_{j}(t)$ für unterschiedliche, den verschiedenen Greifmustern zugeordneten Referenzobjekte unterschiedlicher Größe. Bei Einsatz einer Momentenregelung sind die Kontaktkräfte unter Berücksichtigung der Fingerkinematik in Gelenkmomente $M_{j}$ umzurechnen. Dieses Verfahren jedoch aber keine Messung des Steifigkeitsoder Impedanzverhaltens der Gelenke. Es ist also nicht bekannt, mit welchem Momentenverhalten die Hand auf externe Kräfte reagiert. Ein Ansatz zur Impedanzbestimmung von Fingern mit Hilfe eines pulsierenden Zylinders und eines Beschleunigungssensors ist in [64, 65] beschrieben. Das Verfahren ist jedoch sehr aufwändig und erlaubt nur die Messung einzelner Finger an bestimmten Arbeitspunkten.

\subsection{Handsteuerung}

Die in Abbildung 5.26 schematisch dargestellte Handsteuerung übernimmt als letzte Ebene oberhalb der Hardware der Hand die Ausführung des gewählten Greifmusters, die Koordination auf der Ebene der Finger und der einzelnen Gelenke, die Regelung der Gelenke und die Überwachung der Gelenkregler [110, 111, 129].

Während eines Greifvorgangs dienen die Greifprimitive als Führungsgrößen für die einzelnen Gelenkregler. Sie sind die Sollvorgabe unter der Annahme, dass das tatsächlich zu greifende Objekt genau dem verwendeten Demonstrationsobjekt entspricht. Dieser Fall ist jedoch eher als seltene Ausnahme zu betrachten. Daher soll sich die Hand möglichst gut an die aktuelle Greifsituation anpassen und Abweichungen in einem bestimmten Rahmen tolerieren können. Das kann mit den 


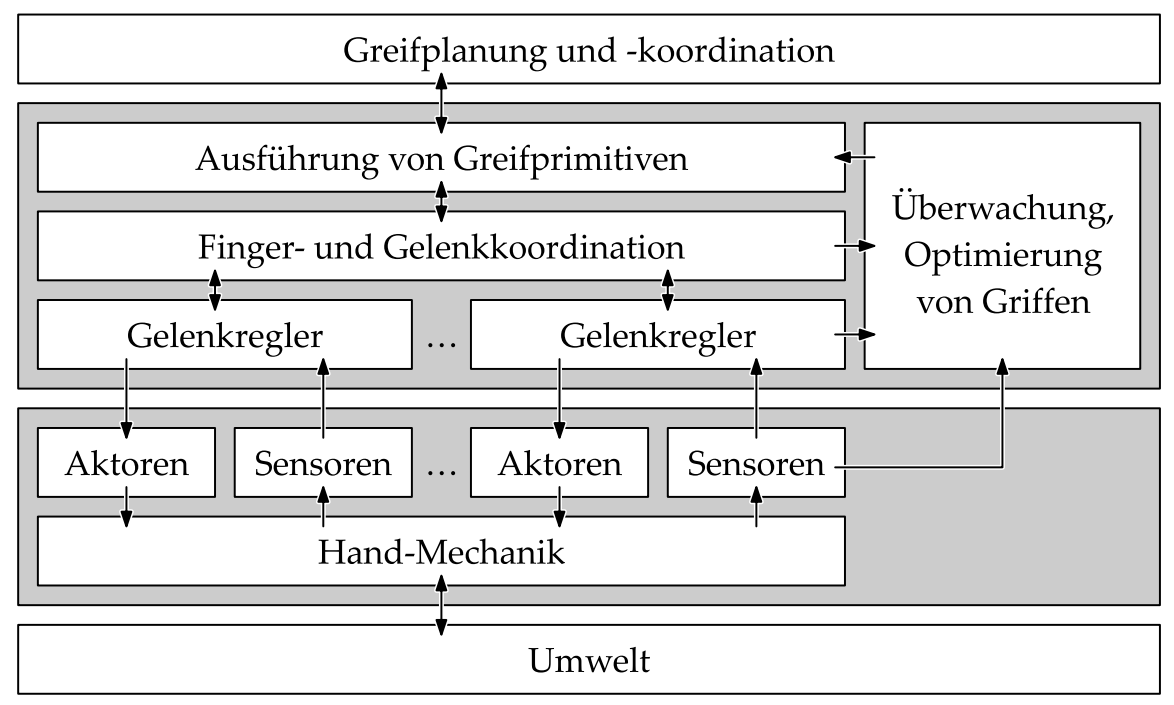

Abbildung 5.26: Hierarchie von Handsteuerung und -hardware.

im Abschnitt 5.3 neu erprobten Verfahren zur Steuerung und Anpassung des Positionsregelungsverhaltens und den im Abschnitt 5.4 hergeleiteten Methoden zur Gelenkpriorisierung erreicht werden.

\subsection{Hand- Arm- Koordination}

Die Planungs- und Koordinationsebene für den Arm übernimmt die Aufgabe, aus einer vorgegebenen Zielposition und -ausrichtung der Hand die dazugehörigen Gelenktrajektorien zu bestimmen. Dabei sind Randbedingungen wie zu vermeidende Kollisionen, Mindestabstände sowie Redundanzen und Restriktionen der Armkinematik zu berücksichtigen. Die Armsteuerung führt die geplante Trajektorie aus, indem sie die einzelnen Armgelenke regelt und synchronisiert. Dabei stehen Aspekte wie das dynamische Verhalten des Arms, das sich mit der Armkonfiguration und durch Zuladung ändert, im Vordergrund. An unterster Stelle in dieser Struktur befindet sich die Arm-Hardware mit Ansteuerung, Aktoren, Armmechanik und Sensoren. Als Informationen werden die Stellgrößen für die Aktoren und die Messwerte der Sensoren übermittelt [154].

Ziel der Hand-Arm-Koordination ist es, Armbewegungen und Greifausführung weitgehend unabhängig voneinander ablaufen zu lassen. Dabei soll nur an einzelnen Stellen eine Synchronisation der beiden Vorgänge stattfinden. Offensichtlich ist diese Anforderung z.B. bei der Annäherung an ein zu greifendes Objekt. Hier darf der Arm die Hand erst dann auf das Objekt zufahren, wenn sie ihre Pregrasp-Bewegung abgeschlossen hat. Ansonsten muss mit unerwünschten Kollisionen gerechnet werden. Umgekehrt darf die Hand erst dann ein Objekt wieder loslassen, wenn der Arm die Zielposition erreicht hat.

Für den Arm ergeben sich zu jeder Phase eines Greifvorgangs Randbedingun- 
gen für die Planung und Ausführung ihrer Bewegungen (Tabelle 5.4).

\begin{tabular}{l|l|l|} 
Phase & Armaktivität & Handaktivität \\
\hline $\begin{array}{l}\text { Annäherung } \\
\text { an Objekt }\end{array}$ & $\begin{array}{l}\text { Grobbewegung mit hoher Ge- } \\
\text { schwindigkeit }\end{array}$ & Pregrasp-Bewegung \\
\hline $\begin{array}{l}\text { Anrück- } \\
\text { bewegung }\end{array}$ & $\begin{array}{l}\text { Feinbewegung mit niedriger } \\
\text { Geschwindigkeit, feste räum- } \\
\text { liche Ausrichtung der Hand }\end{array}$ & Warten auf Kontakt \\
\hline Zugreifen & Positionskorrektur & $\begin{array}{l}\text { Hand schließen, Erhöhen der } \\
\text { Greifkraft, Überprüfung der } \\
\text { Greifkonfiguration }\end{array}$ \\
\hline $\begin{array}{l}\text { Bewegen } \\
\text { des Objekts }\end{array}$ & $\begin{array}{l}\text { Feinbewegung mit niedriger } \\
\text { Geschwindigkeit, bei Bedarf } \\
\text { feste räumliche Ausrichtung } \\
\text { der Hand }\end{array}$ & $\begin{array}{l}\text { Halten der Greifkraft, Über- } \\
\text { prüfung der Greifkonfigurati- } \\
\text { on }\end{array}$ \\
\hline
\end{tabular}

Tabelle 5.4: Arm- und Handaktivitäten eines beispielhaften Greifablaufs.

Der Arm hat zum Ende der Grobbewegung in die Nähe des zu greifenden Objekts die Hand erstmals so im Raum auszurichten, dass bei der nachfolgenden Anrückbewegung keine Umorientierung mehr stattfinden muss. In dieser Phase bereitet sich die Hand auf das ausgewählte Greifmuster vor, so wird z.B. für einen Zylindergriff der Daumen in Oppositionsstellung gebracht.

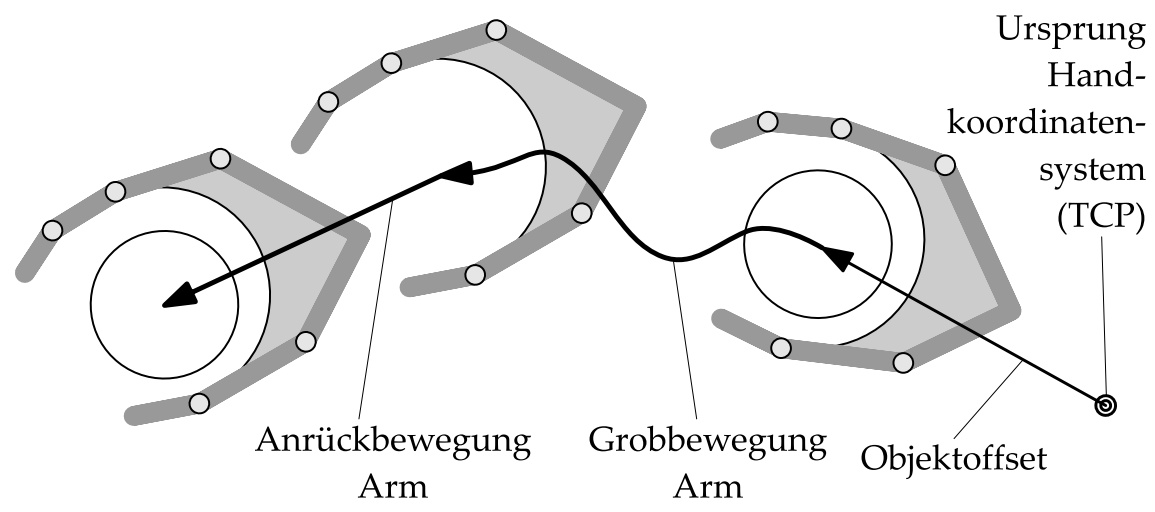

Abbildung 5.27: Schematische Darstellung der Hand- und Objektposition während eines Greifvorgangs.

Während des Anrückens wird die Planungsfreiheit des Arms so eingeschränkt, dass die Hand ohne Änderung ihrer Ausrichtung auf das zu greifende Objekt zu bewegt wird (Abbildung 5.27). Die Randbedingungen sind dabei von der Handsteuerung z.B. als Objektoffset und Anrückvektor relativ zum TCP vorzugeben.

Ist das Objekt gegriffen, dürfen die Restriktionen für die Armbewegung in manchen Fällen nicht aufgehoben werden. So ist z.B. ein mit Wasser gefülltes Glas 
beim Transport aufrecht zu halten. Ist während der Armbewegung eine Ausrichtungsänderung zulässig, so muss das Objekt kurz vor dem Absetzen wieder in eine hierfür geeignete Ausrichtung gebracht werden. 


\section{Zusammenfassung und Ausblick}

Die Mehrheit der heutigen Robotergreifer und Handprothesen besitzt eine geringe Beweglichkeit und eingeschränkte Menschenähnlichkeit. Nur wenige künstliche anthropomorphe Hände kommen dem Vorbild des Menschen nahe. Auf dem Weg zu menschenähnlicheren Händen zum Einsatz in der humanoiden Robotik und der Prothetik ist die Ausstattung mit Sensoren ein wichtiger Baustein.

Das Hauptziel dieser Arbeit ist die Auswahl, Entwicklung und Optimierung von Sensoren für eine von neuartigen flexiblen Fluidaktoren angetriebene künstliche Hand. Demonstriert wird der Aufbau der Sensoren und deren Einsatzmöglichkeiten an Hand von Sensorprototypen. Die Ausstattung mit Sensoren ermöglicht die Regelung und Überwachung der Handaktivitäten und trägt damit zu einer höheren Greifsicherheit und Geschicklichkeit der Hand bei.

Diese Arbeit umfasst die folgenden inhaltlichen Schwerpunkte:

- Zusammenstellung der Anforderungen an die Aufgaben und Fähigkeiten anthropomorpher Hände für einen humanoiden Roboter unter Anlehnung an die Physiologie und Tätigkeiten der menschlichen Hand sowie Auswahl geeigneter Sensorklassen (Kapitel 2)

- Analyse und Modellierung der Momenten- und Volumenkennfelder von in Fingergelenken einsetzbaren flexiblen Fluidaktoren sowie Charakterisierung ihres Momenten- und Federverhaltens (Kapitel 3)

- Darstellung der bereits in künstlichen Händen eingesetzten Messverfahren, Auswahl der für den Anwendungsfall geeigneten Messprinzipien, Entwicklung und Optimierung der Sensoren (Kapitel 4), insbesondere:

- Sensoren zur Messung der Gelenkwinkel basierend auf Hallsensoren und Permanentmagneten (Abschnitt 4.1)

- Piezoresistive taktile Sensoren mit elastischer Oberfläche zur Messung von Greifkräften (Abschnitt 4.2)

- Sensoren für die Messung des Aktordrucks und die Bestimmung daraus ableitbarer Größen (Abschnitt 4.3)

- Bewertung von Leitungsmaterialien und Herleitung einer Verkabelungsstruktur für die Übertragung der Sensorsignale in einem Handprototypen ( $\mathrm{Ab}$ schnitt 4.4) 
- Darstellung verschiedener Regelungsvarianten für einzelne und mehrere Gelenke im pneumatischen und hydraulischen Betrieb sowie Herleitung der Eigenschaften und Funktionen einer Greifsteuerung als Schnittstelle zwischen der Gelenkregelung und einer übergeordneten Robotersteuerung (Kapitel 5)

Die dabei erhaltenen wichtigsten Ergebnisse der Arbeit sind:

1. Konzeption eines modulares Sensorsystems für eine Hand mit flexiblen Fluidaktoren, aufbauend auf der Analyse der menschlichen Hand und der Anforderungen an künstliche anthropomorphe Hände für humanoide Roboter.

2. Mathematische Beschreibung des passiven Nachgiebigkeits- und Steifigkeitsverhaltens der in den Fingergelenken eingesetzten flexiblen Fluidaktoren unter Berücksichtigung der Unterschiedlichkeit des hydraulischen und pneumatischen Betriebs. Diese für das sichere Greifen vorteilhafte Eigenschaft muss bei den meisten anderen Antriebsprinzipien erst durch eine Steuerung oder Regelung erzeugt werden.

3. Auswahl der geeigneten Sensorprinzipien für eine künstliche Hand unter Berücksichtigung von Geometrie und Verhalten der in den Fingergelenken angebrachten flexiblen Aktoren und des Aufbaus der Fingerstruktur. Anpassung, Integration und Test von Winkelsensoren, taktilen Kraftsensoren und Sensoren zur Messung des Aktordrucks.

4. Minaturisierung und Optimierung der Winkelsensoren als Anordnung aus Hallsensor und Permanentmagnet. Durch die Reduzierung der Magnetgeometrie wird eine geringere Schwächung der mechanischen Struktur als bisher erreicht.

5. Entwicklung eines neuen Optimierungsverfahrens für die Außenkontur des Halbringmagneten auf der Basis von genetischen Algorithmen und der FEMSimulation des Magnets zur Kompensation der gestiegenen Nichtlinearität des Sensorsignals.

6. Integration von FSR-Foliensensoren als taktile Sensoren in die nahe an der Handinnenfläche liegende Fingerstruktur. Entwicklung einer nachgiebigen Sensorabdeckung zum Schutz des Sensors und zur Unterstützung des sicheren Greifens. Anpassung der Sensorabdeckung für ein empfindliches Ansprechen im Kraftbereich unter $2 N$ unter Berücksichtigung einer Maximalkraft von $15 N$.

7. Anpassung von Sensoren für die Drucküberwachung der Aktoren, um im hydraulischen Betrieb eine Überlastung durch den von externen Kräften und der fehlenden Kompressibilität verursachten Druckanstieg verhindern zu können. Herleitung und Überprüfung der modellbasierten Schätzung des ausgeübten Gelenkmoments aus dem gemessenen Aktordruck in Verbindung mit Winkelsensoren. 
8. Verifikation der Sensorfunktionen an Hand verschiedener Gelenkregler für den pneumatischen und hydraulischen Betrieb. Modellierung des Schaltverhaltens druckabhängig angesteuerter Digitalventile und seine Nutzung bei der Realisierung eines Druckregelkreises.

9. Konzeption und experimenteller Test der statischen, dynamischen und steifigkeitsabhängigen Momentenbegrenzung des Positionsreglers zur gezielten Beeinflussung des Verhaltens bei Objektkontakt.

10. Ableitung von Vorgehensweisen zur Realisierung eines menschenähnlichen Greifverhaltens im Kontext eines hierarchisch strukturierten Steuerungssystems eines humanoiden Roboters, insbesondere für grundlegende Synchronisationsvorgänge in der Koordination von Arm und Hand.

Für eine Umsetzung als Produkt können die beschriebenen Sensor- und Leitungskomponenten weiter integriert werden, z.B.in eine modulare Sensoreinheit für ein Fingerglied, die auf einer Starr-Flex-Platine den Hallsensor zur Winkelmessung, den FSR-Sensor sowie die Verbindung zum proximalen Fingerglied zusammenfasst. Bei den Drucksensoren kann eine kompakte Messanordung durch die Erweiterung der Ventilbank erreicht werden.

Die Ansätze zur Ausführung von Greifvorgängen mit einem gezielt momentenbegrenzten Positionsregler sind auf Grund ihres geringen Rechenaufwands vielversprechend für die Implementierung in Mikrocontrollern. Hierfür sind als nächste Entwicklungsschritte das Verhalten von Mehrgelenkfingern und Mehrfingergreifern in der Simulation und mit Prototypen zu untersuchen. 


\section{Literaturverzeichnis}

[1] 3M Electronic Products Division: Cable Properties - Internal Wiring. Datenblatt TS-0731-04,www. 3m.com, 2003.

[2] 3M Interconnect Solutions Division: .050", Medium Flex Life Cable, 28 AWG Stranded, PVC, 3539 Series. Datenblatt TS-0058-09, www.3m.com, Februar 1999.

[3] 3M Interconnect Solutions Division: .050", High Flex Life Cable, 28 AWG Stranded, PVC, 3319 Series. Datenblatt TS-0059-11, November 2002.

[4] 3M Interconnect Solutions Division: .025", Round Conductor Flat Cable, 30 AWG Stranded, PVC, 3754 Series. Datenblatt TS-0875-07, www. 3m. com, Januar 2003.

[5] 3M Interconnect Solutions Division: Flex Cycles. Datenblatt TS-073202,www.3m. com, 2003.

[6] AlBU-SCHÄFFER, A.: Regelung von Robotern mit elastischen Gelenken am Beispiel der DLR-Leichtbauarme. Doktorarbeit, Technische Universität München, 2002.

[7] Allegro Microsystems: 3515 and 3516 - Ratiometric, Linear Hall- Effect Sensors for high-temperature Operation. Datenblatt Nr. 27501.10B*, 2000.

[8] American Honda Motor Co., Inc.: ASIMO Technical Information. Datenblatt,www. as imo.honda.com, Januar 2003.

[9] Asfour, T., K. Berns und R. Dillmann: The Humanoid Robot ARMAR: Design and Control. In: Proceedings of the 2000 International Conference on Humanoid Robots, MIT, Boston, USA, September 2000.

[10] Axon Kabel GmbH: Axojump Flexible Flachkabel. Datenblatt, www. axon-cable.com, März 2002.

[11] BECK, S.: Konzeption, Aufbau und Erprobung einer Testumgebung für die Evaluierung flexibler Fluidaktoren. Diplomarbeit, Universität Karlsruhe (TH), 2001.

[12] Beck, S., A. Lehmann, T. Lotz, J. Martin, R. Keppler und R. Mikut: Modellgestützte adaptive Regelungskonzepte für eine fluidisch betriebene Roboterhand. In: GMA-Kongress 2003, Baden-Baden, VDI Bericht Nr. 1756, Seiten 6572. VDI-Verlag, Düsseldorf, 2003. 
[13] BeCK, S., A. LeHMAnN, J. MARTin und R. Mikut: Modellbildung und FuzzyGelenkpositionsregelung für eine 5-Finger-Roboterhand mit flexiblen Fluidaktoren. In: MIKUT, R. und M. ReISCHL (Herausgeber): Proceedings, 12. Workshop Fuzzy Systeme des GMA-FA 5.22., Dortmund, Wissenschaftliche Berichte FZKA 6767, Seiten 177-191. Forschungszentrum Karlsruhe, November 2002.

[14] Beck, S., R. Mikut, A. Lehmann und G. Bretthauer: Model-Based Control and Object Contact Detection for a Fluidic Actuated Robotic Hand. In: ZHU, J. J. (Herausgeber): Proceedings of the 42nd IEEE Conference on Decision and Control, Seiten 6369-6374. IEEE Press, Dezember 2003.

[15] Bernieri, S., A. Caffaz, G. Cannata und G. Casalino: The DisT-Hand Robot. In: Proceedings of the 1997 IEEE/RSJ International Conference on Intelligent Robots and Systems, Grenoble, Frankreich. IEEE Press, September 1997.

[16] BERTELS, T.: Funktionen einer Kraftzugbandage für Prothesen der oberen Extremität. Med. Orth. Tech., 121:13-17, 2001.

[17] Biagiotti, L., F. Lotti, C. Melchiorri und G. VAssura: Design Aspects For Advanced Robot Hands. In: Proceedings of the 2002 IEEE/RSJ International Conference on Intelligent Robots and Systems, Lausanne, Schweiz. IEEE Press, September 2002. Tutorial T1 - Towards Intelligent Robotic Manipulation.

[18] Biagiotti, L., F. Lotti, C. Melchiorri und G. VAssura: Mechatronic Design Of Innovative Fingers For Anthropomorphic Robot Hands. In: Proceedings of the 2003 IEEE International Conference on Robotics and Automation, Taipei, Taiwan, Seiten 3187-3192. IEEE Computer Society Press, September 2003.

[19] BICCHI, A.: A Criterion for Optimal Design of Multiaxis Force Sensors. Journal of Robotics and Autonomous Systems, 10(4), 1992.

[20] BICcHI, A.: Hands for Dexterous Manipulation and Robust Grasping: A Difficult Road Toward Simplicity. IEEE Transactions on Robotics and Automation, 16(6):652-662, 2000.

[21] BorovaC, B., D. ŠEŠLIJA und S. STANKOvSKI: Soft Sensored Grippers in Assembly Process. In: Proceedings of the 1992 IEEE International Conference on Robotics and Automation, Nizza, Frankreich, Seiten 1283-1288. IEEE Computer Society Press, 1992.

[22] Butterfass, J., M. Grebenstein, H. Liu und G. Hirzinger: DLRHand II: Next Generation of a Dextrous Robot Hand. In: Proceedings of the 2001 IEEE International Conference on Robotics and Automation, Seoul, Korea, Seiten 109-114. IEEE Computer Society Press, 2001.

[23] Butterfass, J., G. Hirzinger, S. KNOCH und H. LiU: DLR's Multisensory Hand Part I: Hard-and Software Architecture. In: Proceedings of the 1998 IEEE International Conference on Robotics and Automation, Leuven, Belgien, Seiten 2081-2086. IEEE Computer Society Press, 1998. 
[24] ButTERfASS, J.: Eine hochintegrierte multisensorielle Vier-Finger-Hand für Anwendungen in der Servicerobotik. Doktorarbeit, Technische Universität Darmstadt, 2000.

[25] CAfFaZ, A. und G. CANnAtA: The design and Development of the DIST-Hand Dextrous Gripper. In: Proceedings of the 1998 IEEE International Conference on Robotics and Automation, Leuven, Belgien, Seiten 2075-2080. IEEE Computer Society Press, 1998.

[26] Caldwell, D. G. und N. TsagaRAKIS: Soft grasping using a dextrous hand. Industrial Robot: An international Journal, 27(3):194-199, 2000.

[27] Carrozza, M. C., B. Massa, S. Micera, M. Zecca und P. Dario: A 'Wearable' Artificial Hand for Prosthetics and Humanoid Robotics Applications. In: Proceedings of the 2001 International Conference on Humanoid Robots, Waseda University, Tokio, Japan, November 2001.

[28] Carrozza, M. C., S. Micera, B. Massa, M. Zecca, R. Lazzarini, P. CANELLI und P. DARIO: The Development of a Novel Biomechatronic Hand - Ongoing Research and Preliminary Results. In: Proceedings of the 2001 IEEE/ASME International Conference on Advanced Intelligent Mechatronics, Como, Italien, Seiten 249-254. IEEE Press, Juli 2001.

[29] Castro, M. C. F. und A. Cliquet: A Low-Cost Instrumented Glove for Monitoring Forces During Object Manipulation. IEEE Transactions on Rehabilitation Engineering, 5(2), Juni 1997.

[30] Chatterjee, S. und B. Price: Praxis der Regressionsanalyse. Oldenbourg Verlag, 2. Auflage, 1995.

[31] ConexCon Inc.: 9063 Series 0.635 mm Center Spacing Flat Cable. Datenblatt, WwW. conexcon.com, 2003.

[32] Craelius, W.: The Bionic Man: Restoring Mobility. Science, 295:1018-1021, 2002.

[33] Craelius, W., R. L. Abboudi und N. A. Newby: Control of a Multi-finger Prosthetic Hand. In: ICORR '99: International Conference on Rehabilitation Robotics, Stanford, CA, Seiten 255-260, 1999.

[34] Cutkosky, M. R.: On Grasp Choice, Grasp Models, and the Design of Hands for Manufacturing Tasks. IEEE Transactions on Robotics and Automation, 5(3):269-279, Juni 1989.

[35] Cutkosky, M. R. und R. D. Howe: Human Grasp Choice and Robotic Grasp Analysis. In: Venkataraman, S. T. und T. Iberall (Herausgeber): Dextrous Robot Hands, Seiten 5-31. Springer Verlag, 1990. 
[36] CutKosky, M. R., J. M. Jourdain und P. K. Wright: Skin Materials for Robotic Fingers. In: Proceedings of the 1987 IEEE International Conference on Robotics and Automation, Raleigh, USA, Seiten 1649-1654. IEEE Computer Society Press, 1987.

[37] CZINKI, A.: Konstruktion, Aufbau und Regelung servopneumatischer Roboterhände. Doktorarbeit, Institut für fluidtechnische Antriebe und Steuerungen (IFAS), Aachen, 2001.

[38] Daerden, F.: Conception and Realization of Pleated Pneumatic Artificial Muscles and their Use as Compliant Actuation Elements. Doktorarbeit, Vrije Universiteit Brussel, Belgien, 1999.

[39] DohtA, S., Y. BAN und H. Matsushita: Application of A Flexible Strain Sensor to a Pneumatic Rubber Hand. In: Proceedings of the 6th Triennal International Symposium on Fluid Control, Measurement and Visualization, Sherbrooke, Quebec, Kanada, August 2000.

[40] DolL, T. J.: Entwicklung und Programmierung einer Roboterhand. Doktorarbeit, Universität Karlsruhe (TH), 1989.

[41] Doll, T. J. und H.-J. Schneebeli: The Karlsruhe Hand. In: Proceedings of the IFAC Symposium on Robot Control, Karlsruhe, Seiten 383-388, 1988.

[42] Douta, K., C. MiyaAKe und K. MiYaKe: Development of Flexible Printed Circuit Boards using Fatigue Life Prediction. Nitto Technical Report, 39(1), Juli 2001.

[43] DÖRSAM, T. H.: Intelligente Steuerungsansätze für mehrfingrige Robotergreifer. Doktorarbeit, Universität Karlsruhe (TH), 1997.

[44] Ebersoldt, A.: Dezentrale Datenerfassung mit Single-Chip-Microcontrollern. Praxisbericht, Berufsakademie Karlsruhe, März 2002.

[45] Ehrenmann, M., O. Rogalla, R. Zöllner und R. Dillmann: Belehrung komplexer Aufgaben für Serviceroboter: Programmieren durch Vormachen im Werkstätten- und Haushaltsbereich. In: DillmanN, R., R. D. SCHRAFT und H. WÖRN (Herausgeber): Robotik 2002 Leistungsstand - Anwendungen - Visionen - Trends, VDI-Bericht Nr. 1679, Seiten 211-218. VDI/VDE- Gesellschaft Mess- und Automatisierungstechnik, 2002.

[46] ENGEL, J., J. CHEN und C. LiU: Development of polyimide flexible tactile sensor skin. Journal of Micromechanics and Microengineering, 13(3):359-366, Mai 2003.

[47] FeARING, R. S.: Tactile Sensing Mechanisms. The International Journal of Robotics Research, 9(3), Juni 1990.

[48] Ferraresi, C., A. M. Bertetto und L. Mazza: Positioning Model of a Flexible Pneumatic Actuator. In: Proceedings of 6th International Workshop on Robotics in Alpe-Adria-Danube Region, Cassino, Italien, Seiten 469-474, 1997. 
[49] Ferrari De CAStro, M. C. und A. Cliquet Jr.: An artificial grasping evaluation system for the paralysed hand. Medical \& Biological Engineering \& Computing, 38:275-280, 2000.

[50] Festo AG \& Co.: Fluidic Muscle MAS Produktdatenblatt. Datenblatt, www. festo.com, 2003.

[51] FISCHER, T.: Sensor system for controlling a multifingered gripper on a robot arm. In: Proceedings of the 1998 IEEE/RSJ International Conference on Intelligent Robots and Systems (IROS 1998), Victoria, B.C., Kanada. IEEE Press, Oktober 1998 1998.

[52] FISCHER, T.: Multisensorbasierte Kraft-Positionsregelung von Mehrfingergreifern. Doktorarbeit, Universität Karlsruhe (TH), 2000.

[53] FISCher, T., D. RAPElA und H. WÖRn: Joint Controller for the Object-Pose Controlling on Multifinger Grippers. In: Proceedings of the 1999 IEEE/ASME International Conference on Advanced Intelligent Mechatronics, Atlanta, Georgia, USA. IEEE Press, September 1999.

[54] Fischer, T., D. RApela und H. WÖRn: Sensor-Based Controlling of the Objects' Pose for Multifinger Grippers. In: IEEE International Symposium on Intelligent Control, 1999.

[55] Fischer, T. und J. SeYfried: The new Karlsruhe Dexterous Hand II. In: International Symposium on Intelligent Robotic Systems, 1997.

[56] FISCHER, T. und H. WÖRN: Structure of a robot system: Karlsruhe Dextrous Hand II. In: Mediterranean Conference on Control and Systems, 1998.

[57] Friedrich, H., V. GrossmanN, M. EhrenmanN, O. Rogalla, R. ZÖllNER und R. DILLMANN: Towards cognitive elementary operators: grasp classification using neural network classifiers. In: Proceedings of the IASTED International Conference on Intelligent Systems and Control (ISC), 28.-30.10.1999, Santa Barbara, Kalifornien, USA, Seiten 121-126, 1999.

[58] FujIKurA Europe LtD.: Flat Flexible Cables Data Sheet. Www. fujikura-westbury.co.uk, 2003.

[59] Fukaya, N., N. Toyama, T. Asfour und R. Dillmann: Design of the TUAT / Karlsruhe Humanoid Hand. In: Proceedings of the 2000 IEEE/RSJ International Conference on Intelligent Robots and Systems, Takamatsu, Japan. IEEE Press, Oktober 2000.

[60] Gazeau, J.-P., S. Zeghloul, M. Arsicault und J.-P. Lallemand: The LMS Hand: force and position control in the aim of the fine manipulation of objects. In: Proceedings of the 2001 IEEE International Conference on Robotics and Automation, Seoul, Korea, Seiten 2642-2648. IEEE Computer Society Press, 2001. 
[61] Gentile, C. T., M. Wallace, T. D. Avalon, S. Goodman, R. Fuller und T. HAlL: Angular Displacement Sensors, US Patent Nr. 5,086,865, Februar 1992.

[62] Golubovic, D.: Modellbildung und Entwurf einer Druckregelung für pneumatisch betriebene flexible Fluidaktoren. Diplomarbeit, Universität Karlsruhe (TH), 2003.

[63] Grupen, R. A., T. C. Henderson und I. D. MCCAmmon: A survey of general purpose manipulation. International Journal of Robotics Research, 8(1), 1989.

[64] HajIAN, A. Z.: A characterization of the mechanical impedance of human hands. Doktorarbeit, Harvard University, Cambridge, Massachusetts, USA, Juni 1997.

[65] HaJIAN, A. Z. und R. D. HOWE: Identification of the mechanical impedance at the human finger tip. ASME Journal of Biomechanical Engineering, 1(119):109-114, Februar 1997.

[66] HARTWIG, J.: Konstruktive Auslegung eines fluidisch angetriebenen Miniaturgelenks und dessen Optimierung mittels FEM für die Realisierung einer anthropomorphen künstlichen Hand. Diplomarbeit, Hochschule für Technik Karlsruhe (FH), 2002.

[67] Harvey, I.: Artificial Evolution: A Continuing SAGA. In: GOMI, T. (Herausgeber): Evolutionary Robotics: From Intelligent Robots to Artificial Life. Proceedings of the 8th International Symposium on Evolutionary Robotics, Tokio, Japan, Lecture Notes in Computer Science 2217. Springer-Verlag, 2001.

[68] Hashimoto, H., H. Ogawa, M. Obama, T. Umeda und K. Tatsuno: A Multi-Fingered Hand with Newly Developed Tactile Sensors. Journal of Robotics and Mechatronics, Tokio, 5(1):60-65, 1993.

[69] Heckathorne, C. W.: Characterization of a Force-Sensitive Resistive Transducer for Application to Prosthesis Control. In: Proceedings of the 12th Annual Conference on Rehabilitation Technology, New Orleans, Louisiana, Seiten 224-225, Juni 1989.

[70] Hirai, S., P. Cusin, H. Tanigawa, T. Masui, S. Konishi und S. KaWAMURA: Qualitative Synthesis of Deformable Cylindrical Actuators through Constraint Topology. In: Proceedings of the 2000 IEEE/RSJ International Conference on Intelligent Robots and Systems, Takamatsu, Japan, Seiten 197-202. IEEE Press, Oktober 2000.

[71] Hirai, S., T. Masuo und S. Kawamura: Prototyping Pneumatic Group Actuators Composed of Multiple Single-motion Elastic Tubes. In: Proceedings of the 2001 IEEE International Conference on Robotics and Automation, Seoul, Korea, Seiten 3807-3812. IEEE Computer Society Press, 2001. 
[72] Hirukawa, H., F. Kanehiro, K. KaneKo, S. Kajita, K. FujiWARA, Y. KaWAI, F. TOMITA, S. HiRAi, K. TANIE, T. ISOZUMI, Y. AKACHI, T. KAWASAKI, S. OTA, K. YOKOHAMA, H. HANDA, Y. FuKaSE, J. MAEdA, Y. NAKAMURA, S. TACHI und H. INOUE: Humanoid Robotics Platforms developed in HRP. In: Proceedings of the 2003 International Conference on Humanoid Robots, Karlsruhe und München (CD-ROM), Seite 5, Oktober 2003.

[73] HoGAN, N.: Impedance control: An approach to manipulation, part I - theory, part II - implementation, part III - applications. Journal of Dynamic Systems, Measurement and Control, 107:1-24, März 1985.

[74] Honeywell Inc.: 24PC Series Pressure Sensors, Absolute Unamplified Noncompensated. Interactive Catalog, www.honeywell.com/sensing, 2003.

[75] Howe, H. D. und M. R. CUTKOSKY: Sensing skin acceleration for slip and texture perception. In: Proceedings of the 1989 IEEE International Conference on Robotics and Automation, Scottsdale, Arizona, USA, Seiten 145-150. IEEE Computer Society Press, 1989.

[76] Howe, R., N. Popp, P. Akella, I. KaO und M. R. CutKosky: Grasping, Manipulation and Control with Tactile Sensing. In: Proceedings of the 1990 IEEE International Conference on Robotics and Automation, Cincinnati, Ohio, USA, Seiten 1258-1263. IEEE Computer Society Press, 1990.

[77] HRISTU, D., N. FERRIER und R. W. BROCKETT: The performance of a deformable-membrane tactile sensor: basic results on geometrically-defined tasks. In: Proceedings of the 2000 IEEE International Conference on Robotics and Automation, San Francisco, Kalifornien, USA, Band 1, Seiten 508-513. IEEE Computer Society Press, 2000.

[78] IEE INTERNATIONAL ELECTRONICs \& ENGINEERING: FSR-Sensoren: Daten, Eigenschaften und Hinweise zur Handhabung, März 1998.

[79] Immersion INC.: Cyberglove. Datenblatt, 2002.

[80] Jacobsen, S. C., D. F. Knutti, J. E. Wood und K. B. Biggers: The UTAH/MIT Dextrous Hand: Work in Progress. The International Journal of Robotics Research, 3(4):21-50, 1984.

[81] JacobSen, S. C. ET AL.: Design of the UTAH/M.I.T. Dextrous Hand. In: Proceedings of the 1986 IEEE International Conference on Robotics and Automation, San Francisco, USA, Seiten 1520-1532. IEEE Computer Society Press, 1986.

[82] JENSEN, T. R., R. G. RADWIN und J. G. WEBSTER: A conductive polymer sensor for measuring external finger forces. Journal of Biomechanics, 24(9), 1991.

[83] JOCKUSCH, J.: Taktile Sensorik für eine Roboterhand. Diplomarbeit, Technische Fakultät der Universität Bielefeld, 1996. 
[84] JocKUSCH, J.: Exploration based on neural networks with applications in manipulator control. Doktorarbeit, Technische Fakultät der Universität Bielefeld, 2001.

[85] JOCKUSCH, J., J. WALTER und H. RITTER: A Tactile Sensor System for a ThreeFingered Robot Manipulator. In: Proceedings of the 1997 IEEE International Conference on Robotics and Automation, Albuquerque, USA, Band 4, Seiten 3080-3086. IEEE Computer Society Press, 1997.

[86] Johnston, D., P. Zhang, J. Hollerbach und S. Jacobsen: A Full Tactile Sensing Suite for Dextrous Robot Hands and Use in Contact Force Control. In: Proceedings of the 1996 IEEE International Conference on Robotics and Automation, Minneapolis, USA, Seiten 3222-3227. IEEE Computer Society Press, 1996.

[87] J.S.T. JAPANESE SOlderless Terminals MFG. Co., LtD., OsaKa, JAPAN: JST Catalog Vol. 117e. Katalog, www. jst.com, 2002.

[88] KANG, S. B.: Robot Instruction by Human Demonstration. Doktorarbeit, The Robotics Institute, Carnegie Mellon University, USA, 1994.

[89] KapandjI, I. A. (Herausgeber): Funktionelle Anatomie der Gelenke, Band 1: Obere Extremität. Ferdinand Enke Verlag, Stuttgart, 2. Auflage, 1992.

[90] Kargov, A., C. Pylatiuk, J. Martin und S. Schulz: A Comparison of the Grip Force Distribution in Natural Hands and in Prosthetic Hands. In: Abstracts, 2nd World Congress of the International Society of Physical and Rehabilitation Medicine (ISPRM), Prag, Tschechische Republik, Seite 76, Mai 2003.

[91] Kargov, A., C. Pylatiuk, J. Martin und S. Schulz: A comparison of the prehension force requirements in prosthetic hands. In: Book of Abstracts, 15th International Interbor Congress on Prosthetics and Orthotics, Budapest, Ungarn, Seiten 52-53, Juni 2003.

[92] Kargov, A., C. Pylatiuk, J. Martin und S. Schulz: Determination of the Grip Force Distribution in Functional Grasping. In: Abstracts, 7th Conference of the European Society for Engineering and Medicine (ESEM), Halle (Saale), Seite 64, September 2003.

[93] Kargov, A., C. Pylatiuk, J. Martin und S. Schulz: Determination of the Grip Force Distribution in Functional Grasping. Technology and Health Care, 12(2):193-194, 2004.

[94] Kargov, A., C. Pylatiuk, J. Martin, S. Schulz und L. Döderlein: A comparison of the Grip Force Distribution in Natural Hands and in Prosthetic Hands. Disability and Rehabilitation, 2004. (Angenommener Beitrag).

[95] KaWAsaki, H., T. Mouri, J. TAKai und S. ITO: Grasping of Unknown Object imitating Human Grasping Reflex. In: Proceedings of the 15th IFAC World Congress on Automatic Control, 12-26. Juli 2002, Barcelona, Spanien. Elsevier Science, 2002. 
[96] KaWASAKI, H., H. SHIMOMURA und Y. SHImizU: Educational-industrial complex development of an anthropomorphic robot hand 'Gifu hand'. Advanced Robotics, Utrecht, 15(3):357-363, 2001.

[97] Keller AG fÜr Druckmesstechnik: Piezoresistive Low Cost Pressure Sensor, Series 1 TAB. Datenblatt, www.keller-druck.ch, April 1999.

[98] Kinoshita, G., Y. Kurimoto, H. Osumi und K. Umeda: Dynamic Contact Sensing of Soft Planar Fingers with Tactile Sensors. In: Proceedings of the 2001 IEEE International Conference on Robotics and Automation, Seoul, Korea, Seiten 565-570. IEEE Computer Society Press, 2001.

[99] Klute, G. K.: Accounting for Elastic Energy Storage in McKibben Artificial Muscle Actuators. ASME Journal of Dynamic Systems, Measurement, and Control, 122(2):386-388, 2000.

[100] Klute, G. K., J. M. Czerniecki und B. Hannaford: McKibben Artificial Muscles: Pneumatic Actuators with Biomechanical Intelligence. In: Proceedings of the 1999 IEEE/ASME International Conference on Advanced Intelligent Mechatronics, Atlanta, Georgia, USA. IEEE Press, September 1999.

[101] Klute, G. K. und B. HannAford: Fatigue Characteristics of McKibben Artificial Muscle Actuators. In: Proceedings of the 1998 IEEE/RSJ International Conference on Intelligent Robots and Systems (IROS 1998), Victoria, B.C., Kanada. IEEE Press, Oktober 19981998.

[102] KRAUT, D.: Auswahl und Vermessung von Kraftsensoren zur Integration in eine künstliche anthropomorphe Hand. Praxisbericht, Berufsakademie Karlsruhe, März 2002.

[103] KYBERD, P. J. und P. H. CHAPPELL: A force sensor for automatic manipulation based on the Hall effect. Measuring Science and Technology, 4:281-287, 1993.

[104] Kyberd, P. J. und P. H. Chappell: The Southampton Hand: An intelligent myoelectric prosthesis. Journal of Rehabilitation Research and Development, 31(4):326-334, 1994.

[105] LedermanN, S. J., R. L. KLATZKY und D. T. PAWLUK: Lessons from the Study of Biological Touch for Robotic Haptic Sensing. In: NICHOLLS, H. R. (Herausgeber): Advanced Tactile Sensing for Robotics, Seiten 193-220. World Scientific, 1992.

[106] Ledermann, S. J. und D. T. PAWluk: Lessons from the Study of Biological Touch for Robotic Tactile Sensing. In: NicHOLLS, H. R. (Herausgeber): Advanced Tactile Sensing for Robotics, Seiten 151-192. World Scientific, 1992.

[107] LEE, K.-M., J. JONI und X. YIN: Compliant Grasping Force Modeling for Handling of Live Objects. In: Proceedings of the 2001 IEEE International Conference on Robotics and Automation, Seoul, Korea, Seiten 1059-1064. IEEE Computer Society Press, 2001. 
[108] LeE, M. H.: Tactile Sensing: New Directions, New Challenges. The International Journal of Robotics Research, 19(7):636-643, 2000.

[109] LEE, M. H. und H. R. NiCHOLLS: Tactile sensing for mechatronics-a state of the art survey. Mechatronics, 9(1):1-31, 1999.

[110] Lehmann, A., J. Martin, R. Mikut und G. Bretthauer: Konzepte zur Regelung und Stabilitätsüberwachung beim Greifen mit flexiblen Robotergreifern. In: MikUt, R. und M. ReIsCHL (Herausgeber): Proceedings, 13. Workshop Fuzzy Systeme des GMA-Fachausschuss 5.22., Dortmund, Wissenschaftliche Berichte FZKA 6900, Seiten 79-98. Forschungszentrum Karlsruhe, November 2003.

[111] Lehmann, A., R. Mikut, J. Martin und G. Bretthauer: OnlineStabilitätsüberwachung strukturvariabler Roboterregelungen. In: KNOLL, A. (Herausgeber): Robotik 2004 Leistungsstand - Anwendungen - Visionen - Trends, VDI-Bericht Nr. 1841, Seiten 55-62. VDI/VDE- Gesellschaft Mess- und Automatisierungstechnik, 2004.

[112] LiN, L.-R. und H.-P. HuANG: Mechanism Design of a New Multifingered Robot Hand. In: Proceedings of the 1996 IEEE International Conference on Robotics and Automation, Minneapolis, USA, Seiten 1471-1476. IEEE Computer Society Press, 1996.

[113] Liu, H., J. Butterfass, S. KnOCH, P. Meusel und G. Hirzinger: A New Control Strategy for DLR's Multisensory Articulated Hand. IEEE Control Systems, 19(2):47-54, April 1999.

[114] LiU, H., P. Meusel und G. Hirzinger: A Tactile Sensing System for the DLR Three Finger Robot Hand. In: Proceedinges of the 4th International Symposium on Measurement and Control in Robotics (ISMCR '95), Smotenice Castle, Slovakia, Seiten 91-96, 1995.

[115] Loomis, J. M. und S. J. Lederman: Tactual Perception. In: Boff, K., L. KaUfman und J. THOMAS (Herausgeber): Handbook of Perception and Human Performance, Band 2: Cognitive processes and performance. John Wiley \& Sons, New York, 1986.

[116] Loose, T.: Aufbau und Integration der Positionsregelung für eine fluidische Handprothese. Diplomarbeit, Universität Karlsruhe (TH), 2001.

[117] Lotti, F., P. Tiezzi, G. Vassura, L. Biagiotti und C. Melchiorri: UBH 3: An Anthropomorphic Hand With Simplified Endo-Skeletal Structure And Soft Continuous Fingerpads. In: Proceedings of the 2004 IEEE International Conference on Robotics and Automation, New Orleans, Louisiana, USA. IEEE Computer Society Press, April 2004.

[118] LOtTI, F. und G VASSURA: A novel approach to mechanical design of articulated fingers for robotic hands. In: Proceedings of the 2002 IEEE/RSJ International Conference on Intelligent Robots and Systems, Lausanne, Schweiz, Band 2, Seiten 1687-1692. IEEE Press, September 2002. 
[119] Loтz, T.: Modellbildung und Reglerentwurf für die Fingergelenke einer künstlichen Hand mit pneumatischen Fluidaktoren. Diplomarbeit, Universität Karlsruhe (TH), 2003.

[120] LovchiK, C. S. und M. A. DifTler: The Robonaut Hand: A Dexterous Robot Hand for Space. In: Proceedings of the 1999 IEEE International Conference on Robotics and Automation, Detroit, Michigan, USA, Seiten 907-912. IEEE Computer Society Press, 1999.

[121] Lundborg, G., B. Rosen, K. Lindström und S. Lindberg: Artificial Sensitivity based on the use of piezoresistive Sensors. Journal of Hand Surgery, 23 B(5):620-626, 1998.

[122] MaAlej, N., S. Bhat, H. Zhu, G. Webster, W.J. TOMPKins, J. J. Wertsch und P. BACH-Y RITA: A conductive polymer pressure sensor. In: Proceedings of the IEEE Engineering in Medicine and Biology Society 10th Annual International Conference, Seiten 770-771. IEEE Press, 1988.

[123] Martin, J., S. Beck, A. Lehmann, R. Mikut, C. Pylatiuk, S. Schulz und G. Bretthauer: Sensors, Identification and Low Level Control of a Flexible Anthropomorphic Robot Hand. In: Proceedings of the 2003 International Conference on Humanoid Robots, Karlsruhe und München (CD-ROM), Seite 4, Oktober 2003.

[124] Martin, J., S. Beck, A. Lehmann, R. Mikut, C. Pylatiuk, S. Schulz und G. BRETTHAUER: Sensors, Identification and Low Level Control of a Flexible Anthropomorphic Robot Hand. International Journal of Humanoid Robotics, 2004. (Eingeladener Beitrag, im Druck).

[125] Martin, J., R. Keppler, D. Osswald, W. Burger, K. Regenstein, G. Bretthauer, J. Wittenburg, H. Wörn, A. Albers und K. Berns: Mechatronische Konzepte zur Verbesserung der Mensch-Maschine-Interaktion. In: Tagungsbeiträge der Human Centered Robotic Systems, Karlsruhe, Deutschland, Seiten 65-72, Dezember 2002.

[126] Meeker, D.: Finite Element Method Magnetics (FEMM) User's Manual, Version 3.3. femm.berlios.de, Mai 2003.

[127] Mentzel, M., F. Hofmann, T. Ebinger, J. GÜlKe, L. Kinzl und N. J. WACHTER: Kraftmessung an der Hand mit einem Sensorhandschuh bei Griffen mit submaximaler und maximaler Kraft. Handchir Mikrochir plast Chir, Seiten 52-57, 2001.

[128] Menzel, R., K. Woelfl und F. Pfeiffer: The development of a hydraulic hand. In: Proceedings of the 2nd Conference on Mechatronics and Robotics, Seiten 225238, 1993.

[129] Mikut, R., A. Lehmann und G. Bretthauer: Fuzzy Stability Supervision of Robot Grippers. In: Proceedings of the IEEE International Conference on Fuzzy Systems (FUZZ-IEEE), Budapest, 2004. (Angenommener Beitrag). 
[130] Minco InC.: Flex-Circuit Design Guide. Application Aid 24, www.minco. com, März 2000.

[131] Minco Inc.: Designing for Flexibility and Reliability. Application Aid 31, www.minco.com, Oktober 2001.

[132] Minco INC.: Flex Circuits Bulletin FC-301(A). www.minco.com, Dezember 2002.

[133] Mishra, B. und N. SILVER: Some Discussion of Static Gripping and Its Stability. IEEE Transactions on Systems, Man, and Cybernetics, 19(4):783-796, Juli 1989.

[134] Moss-Salentijn, L.: The Human Tactile System. In: Nicholls, H. R. (Herausgeber): Advanced Tactile Sensing for Robotics, Seiten 123-150. World Scientific, 1992.

[135] MOUNIER, S.: Entwicklung einer realitätsnahen Kraftrückkopplung bei fluidisch betriebenen Handprothesen. Doktorarbeit, Universität Karlsruhe (TH), 2004.

[136] NAPIER, J.: The prehensile movements of the human hand. Journal of Bone and Joint Surgery, 38B(4):902-913, November 1956.

[137] NAPIER, J.: Hands. Princeton University Press, 1993.

[138] Negishi, K.: Double-Acting Flexible Wall Actuator, US Patent 5,067,390, November 1991.

[139] NicholLs, H. R. (Herausgeber): Advanced Tactile Sensing for Robotics. World Scientific, 1992.

[140] NishibORI, K., K. IsHit, M Konishi und K. Oshima: Cylindrical Tactile Sensor for Robot Hand Using Position Sensitive Detectors. Journal of the Robotics Society of Japan (JRSJ), 19(7):82-87, Oktober 2001.

[141] NÖLKeR, C. und H. RITTER: GREFIT: Visuelle Erkennung kontinuierlicher Handposturen. In: Informatik '98: Informatik zwischen Bild und Sprache, Magdeburg, Seiten 213-222. Springer Verlag, 1998.

[142] NÖlKer, C. und H. RitTer: GREFIT: Visual Recognition of Hand Postures. In: BrafFort, A., R. GHERbi, S. Gibet, J. RichardSON und D. TEIL (Herausgeber): Gesture-Based Communication in Human-Computer Interaction, Proceedings of the International Gesture Workshop, Gif-sur-Yvette, Frankreich, Seiten 61-72. LNAI 1739, Springer Verlag, 1999.

[143] Noritsugu, T. und T. TANAKa: Application of Rubber Artificial Muscle Manipulator as a Rehabilitation Robot. IEEE/ASME Transactions on Mechatronics, 2(4), Dezember 1997.

[144] OKada, K., M. Arai und N. TAnabe: High Fatigue Resist Flexible Printed Circuit for HDD. Fujikura Technical Review, 31(1), Januar 2002. 
[145] OKadA, T.: A New Tactile Sensor Design based on Suspension-Shells. In: VeNKATARAman, S. T. und T. Iberall (Herausgeber): Dextrous Robot Hands, Seiten 267-286. Springer Verlag, 1990.

[146] Osswald, D., J. Martin, C. Burghart, R. Mikut, H. WÖrn und G. BRETTHAUER: Integrating a Robot Hand into the Control System of a Humanoid Robot. In: Proceedings of the 2003 International Conference on Humanoid Robots, Karlsruhe und München (CD-ROM), Seite 37, Oktober 2003.

[147] ОтTO Bock ORTHOpÄDISCHE IndustrIE: Otto Bock System-Elektrohand mit Digital Twin-Steuerung. 647H327, Juli 1999.

[148] OtTo Bock ORTHOpÄDISCHE INDUSTRIE: Otto Bock System-Elektrohand mit DMC plus-Steuerung. 647H326, November 1999.

[149] Отto Bock ORTHOPÄDISCHE IndUSTRIE: Patienteninformation für Otto Bock System-Elektrohände. 646D44, Oktober 1999.

[150] PAWLUK, D. T.: A Viscoelastic Model of the Human Fingerpad and a Holistic Model of Human Touch. Doktorarbeit, Harvard University, Cambridge, Massachusetts, USA, Mai 1997.

[151] Popovic, R. S., P. M. DrljacA und C. SchotT: Bridging The Gap Between AMR, GMR, And Hall Magnetic Sensors. In: 23rd International Conference on Microelectronics (MIEL 2002), Nis, Yugoslavia, Seiten 55-58, 2002.

[152] Provancher, W. R. und M. R. CutKosky: Sensing Local Geometry for Dexterous Manipulation. In: SICILIANO, B. und P. DARIO (Herausgeber): Experimental Robotics VIII, The 8th International Symposium, Sant'Angelo d'Ischia, Italien, 2002, Lecture Notes in Control and Information Sciences. Springer Verlag, 2002.

[153] QUIDET, R.: Conception d'un capteur angulaire pour les articulations d'une prothèse de main actionnée par fluide. Diplomarbeit, Ecole Nationale Supérieure d'Ingénieurs de Méchanique et des Microtechniques, Besançon, 2001.

[154] Regenstein, K. und R. DillmanN: Design of an open hardware architecture for the humanoid robot ARMAR. In: Proceedings of the 2003 International Conference on Humanoid Robots, Karlsruhe und München (CD-ROM), Seite 3, Oktober 2003.

[155] Rembold, D., T. Fischer, M. Müller und H. WÖRn: A flexible manipulation system for handling a large variety of objects. In: International Conference on Agile, Intelligent and Computer-Integrated Manufacturing, 1998.

[156] RÖHRDANZ, F.: Modellbasierte automatisierte Greifplanung. Doktorarbeit, Technische Universität Carolo-Wilhelmina Braunschweig, 1997.

[157] Rogalla, O.: Abbildung von Benutzerdemonstrationen auf variable Roboterkonfigurationen. Doktorarbeit, Universität Karlsruhe (TH), 2002. 
[158] Russell, R. A. und S. PARKInSON: Sensing Surface Shape by Touch. In: Proceedings of the 1993 IEEE International Conference on Robotics and Automation, Atlanta, Georgia, USA, Band 1, Seiten 423-428. IEEE Computer Society Press, 1993.

[159] SAKAgUCHI, Y.: Flexible Tubular Wall Actuator with End-Mounted Straing Gauge, US Patent 4,860,639, August 1989.

[160] SaKaguchi, Y., T. TAKagi und Y. IMAMURA: Actuator, European Patent EP 0161750 A1, November 1985.

[161] SaKaguchi, Y., T. TAKagi und Y. IMAMURA: Actuator, European Patent EP 0163370 A1, Dezember 1985.

[162] SAlisbury, J. K.: Kinematic and Force Analysis of Articulated Hands. Doktorarbeit, Stanford University, Kalifornien, USA, 1982.

[163] SATO, S., M. Shimojo, Y. SEKI, A. TAKAhashi und S. SHIMIZU: Measuring System for Grasping. In: Proceedings of the 5th IEEE International Workshop on Robot and Human Communication (RO-MAN), Tsukuba, Japan, Seiten 292-297. IEEE Press, November 1996.

[164] SCHMidT, Robert F., Gerhard Thews und Florian LANG (Herausgeber): Physiologie des Menschen. Springer Verlag, 28. Auflage, 2000.

[165] SCHULZ, S.: Verfahren zur planaren Herstellung von pneumatischen und von fluidischen Miniaturmanipulatoren, Patent PCT/EP97/01954 / WO 97/39861, Oktober 1997.

[166] SCHUlZ, S.: Konzept einer neuen Adaptiv-Hand-Prothese auf der Basis flexibler Fluidaktoren. Doktorarbeit, Universität Karlsruhe (TH), 2004. (Im Druck).

[167] Schulz, S., H. Besser, S. DÜrR, M. KOHL, W. Pfleging und U. Voges: Hydraulische Muskeln als Antriebseinheit für endoluminale Systeme in der Medizin. In: 3. Statuskolloquium des Projektes Mikrosystemtechnik, Karlsruhe, Wissenschaftliche Berichte FZKA 6080, 1998.

[168] Schulz, S. und G. Bretthauer: A Fluidic Humanoid Robothand. In: Proceedings of the 2001 International Conference on Humanoid Robots, Waseda University, Tokio, Japan, Seiten 133-139, November 2001.

[169] SChulZ, S. und C. PYlatiuK: Künstliche Hand auf der Basis flexibler Fluidaktoren. In: 4. Statuskolloquium des Programms Mikrosystemtechnik, Karlsruhe, Wissenschaftliche Berichte FZKA 6423, Seiten 239-240, 2000.

[170] Schulz, S., C. Pylatiuk und G. Bretthauer: A New Class of Flexible Fluidic Actuators and their Applications in Medical Engineering. Automatisierungstechnik, 47(8):390-395, 1999. 
[171] Schulz, S., C. Pylatiuk und G. Bretthauer: Entwicklung einer künstlichen Hand mittels flexibler Fluidaktoren. In: GMA-Kongress 2001, Baden-Baden, VDI Bericht Nr. 1680, Seiten 143-149. VDI-Verlag, Düsseldorf, 2001.

[172] Schulz, S., C. Pylatiuk und G. Bretthauer: A New Ultralight Anthropomorphic Hand. In: Proceedings of the 2001 IEEE International Conference on Robotics and Automation, Seoul, Korea, Seiten 2437-2441. IEEE Computer Society Press, 2001.

[173] Schulz, S., C. Pylatiuk, J. Martin und G. Bretthauer: Die Anthropomorphe FZK-Hand. In: DillmanN, R., R. D. SCHRAFT und H. WÖRN (Herausgeber): Robotik 2002 Leistungsstand - Anwendungen - Visionen - Trends, VDIBericht Nr. 1679, Seiten 531-536. VDI/VDE- Gesellschaft Mess- und Automatisierungstechnik, 2002.

[174] ShimogA, K. B.: Robot Grasp Synthesis Algorithms: A Survey. The International Journal of Robotics Research (MIT Press), Seiten 230-266, Juni 1996.

[175] Shimojo, M.: Spatial Filtering Characteristic of Elastic Cover for Tactile Sensor. In: Proceedings of the 1994 IEEE International Conference on Robotics and Automation, San Diego, Kalifornien, USA, Seiten 287-292. IEEE Computer Society Press, 1994.

[176] SHIMOJO, M.: Mechanical Filtering Effect of Elastic Cover for Tactile Sensor. IEEE Transactions on Robotics and Automation, 13(1):128-132, 1997.

[177] Shimojo, M., R. Makino, A. Namiki, M. Ishikawa, T. Suzuki und MABUCHI K.: A Sheet-Type Sensor Using Pressure-Conductive Rubber with Electrical- Wire Stitches Method. In: Proceedings of the first IEEE International Conference on Sensors, Orlando, Florida, USA, Seiten 1637-1642. IEEE Press, Juni 2002.

[178] Smagt, P. Van Der, F. Groen und K. Schulten: Analysis and Control of a Rubbertuator Arm. Biological Cybernetics, 75:433-440, 1996.

[179] Smith, B. P. und W. B. Hudson: The Use of Force Sensing Resistors for Muscle Force Measurements. In: Biomedical Sciences Instrumentation, Band 30, Seiten 153-158, 1994.

[180] So, E., H. Zhang und G. YI-SHENG: Sensing Contact with Analog Resistive Technology. In: Proceedings of the IEEE International Conference on Systems, Man, and Cybernetics, Tokio, Japan, Seiten 806-811. IEEE Press, Oktober 1999.

[181] Sumitomo Electric Europe Ltd.: Sumi-Card Cables Data Sheet, 2003.

[182] SUZUMORI, K.: Elastic materials producing compliant robots. Robotics and Autonomous Systems, 18:135-140, 1996.

[183] Suzumori, K., S. IIKURA und H. TANAKa: Development of Flexible Microactuator and Its Application to Robotic Mechanisms. In: Proceedings of the 1992 
IEEE International Conference on Robotics and Automation, Sacramento, Kalifornien, USA, Seiten 1622-1626. IEEE Computer Society Press, 1991.

[184] TAKAgI, T. und Y. SAKAgUCHI: Pneumatic Actuator for Manipulator, European Patent EP 0123558 A2, Oktober 1984.

[185] TOWNSEND, W. T.: The BarrettHand grasper - programmably flexible part handling and assembly. Industrial Robot: An International Journal, 27(3):181-188, 2000 .

[186] Tremblay, M. R. und M. R. CutKosky: Estimating Friction Using Incipient Slip Sensing During a Manipulation Task. In: Proceedings of the 1993 IEEE International Conference on Robotics and Automation, Atlanta, Georgia, USA, Band 1, Seiten 429-434. IEEE Computer Society Press, 1993.

[187] Triesch, J. und C. vON Der Malsburg: Robust Classification of Hand Postures against Complex Backgrounds. In: Proceedings of the Second International Conference on Automatic Face Recognition and Gesture Recognition 1996, Killington, Vermont, USA, Oktober 1996.

[188] Tubiana, R., J.-M. ThOmine und E. MACKIn: Examination of the Hand and upper Limb. W. B. Saunders Company, 1984.

[189] Upasani, A. V., C. KapoOr und D. TeSAR: Survey of Available Sensor Technology for Robotic Hands. In: Proceedings of DETC99: 1999 ASME Design Engineering Technical Conference, Las Vegas, Nevada, USA, September 1999.

[190] Van Ham, R., B. Verrelst, F. Daerden und D. Lefeber: Pressure Control with On-Off Valves of Pleated Pneumatic Artificial Muscles in a Modular OneDimensional Rotational Joint. In: Proceedings of the 2003 International Conference on Humanoid Robots, Karlsruhe und München (CD-ROM), Seite 35, Oktober 2003.

[191] Venkataraman, S. T. und T. Iberall (Herausgeber): Dextrous Robot Hands. Springer Verlag, 1990.

[192] Verrelst, B., R. Van Ham, J. Vermeulen, D. Lefeber und F. Daerden: Concept of Combining Adaptable Passive Behaviour with an Active Control Structure Using Pleated Pneumatic Artificial Muscles for the Bipedal Robot LUCY. In: Proceedings of the 2003 International Conference on Humanoid Robots, Karlsruhe und München (CD-ROM), Seite 12, Oktober 2003.

[193] WALKeR, R.: Design of a Dextrous Hand for advanced CLAWAR applications. In: 6th International Conference on Climbing and Walking Robots and the Support Technologies for Mobile Machines, Catania, Italien, September 2003.

[194] WALter, J.: Rapid Learning in Robotics. Cuvillier Verlag, 1996.

[195] Walter, J. und H. RitTeR: The Neuroinformatics Robot Laboratory. Technischer Bericht, Universität Bielefeld, September 1996. 
[196] Webster, J. G. (Herausgeber): Tactile Sensing for Robotics and Medicine. J. Wiley \& Sons, 1988.

[197] WeRnSteDT, J.: Experimentelle Prozessanalyse. Oldenbourg Verlag, 1989.

[198] WöHLKe, G.: The Karlsruhe Dextrous Hand: Grasp Planning, Programming $\mathcal{E}$ Real Time Control. In: Proceedings of the 1994 IEEE/RSJ International Conference on Intelligent Robots and Systems, München, Deutschland, Seiten 352-359. IEEE Press, 1994.

[199] WoO, E. J.: Application To Robotic Manipulator Sensing. In: WebSTER, J. G. (Herausgeber): Tactile Sensing for Robotics and Medicine, Seiten 265-280. J. Wiley \& Sons, 1988.

[200] Yamada, Y., Y. Iwanaga, M. Fukunaga, N. Fujimoto, E. OHTA, T. MoRIZONO und Y. UMETANI: Highly Soft Viscoelastic Robot Skin with Contact Surface Location Sensing Capability. In: Proceedings of the 32nd ISR (International Symposium on Robotics), Seiten 1237-1242, Mai 2001.

[201] Yun, M. H., K. KotANI und D. Ellis: Using Force Sensitive Resistors to evaluate Hand Tool Grip Design. In: Proceedings of the Human Factors Society 36th Annual Meeting, Seiten 806-810, 1992.

[202] Zimmerman, T. G.: Optical Flex Sensor, US Patent Nr. 4,542,291, September 1985.

[203] Zimmerman, T. G. und J. Z. LANIER: Computer Data Entry and Manipulation Apparatus and Method, US Patent Nr. 4,988,981, Januar 1991.

[204] ZÖLlner, R., O. Rogalla und R. DillmanN: Integration of Tactile Sensors in a Programming by Demonstration System. In: Proceedings of the 2001 IEEE International Conference on Robotics and Automation, Seoul, Korea, Seiten 25782583. IEEE Computer Society Press, 2001.

[205] ZÖllner, R., O. Rogalla, R. Dillmann und M. Zöllner: Understanding Users Intention: Programming Fine Manipulation Tasks by Demonstration. In: Proceedings of the 2002 IEEE/RSJ International Conference on Intelligent Robots and Systems, Lausanne, Schweiz. IEEE Press, September 2002. 


\section{Abbildungsverzeichnis}

1.1 Beispiele für Greifer und eine anthropomorphe Hand. . . . . . . . . . 15

1.2 Beispiele für humanoide Roboterhände und eine Handprothese. . . . 16

1.3 FZK-Hand: Roboterhand und Prothese. . . . . . . . . . . . . . . . 17

1.4 Einordnung von Händen und Greifern nach ihrer Unabhängigkeit. . 18

2.1 Benennung der Knochen und Gelenke der menschlichen Hand. . . . 25

2.2 Kopplung der Freiheitsgrade in der menschlichen Hand. . . . . . . . 26

2.3 Schichtenaufbau und Rezeptoren der unbehaarten Haut. . . . . . . . 26

2.4 Ablauf von Greif- und Manipulationsvorgängen. . . . . . . . . . . . . 29

2.5 Grifftaxonomie für Produktionsumgebungen. . . . . . . . . . . . . . . 31

2.6 Grifftaxonomie für einen humanoiden Roboter. . . . . . . . . . . . . . 32

3.1 Flexible Fluidaktoren nach dem Kontraktionsprinzip. . . . . . . . . . 37

3.2 Flexibler Fluidaktor nach dem Expansionssprinzip. . . . . . . . . . . 38

3.3 Messdaten: $M_{\mathrm{A}}=f(\varphi, p) \ldots \ldots \ldots \ldots \ldots \ldots$

3.4 Messdaten: Volumen $V_{\mathrm{A}}=f(\varphi, p) \ldots \ldots \ldots \ldots$. . . . . . . . . . . . . . 40

3.5 Winkelkennfeld $\varphi(p, m)$ mit $m=$ konstant. . . . . . . . . . 43

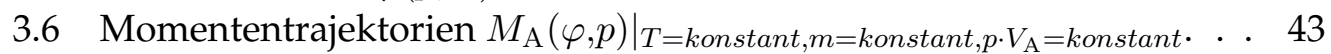

3.7 Winkelkennfeld $\varphi\left(V_{\mathrm{A}}, p\right)$ mit $V_{\mathrm{A}}=$ konstant. $\ldots \ldots \ldots . \ldots 44$

3.8 Momententrajektorien $M_{\mathrm{A}}(\varphi, p)$ mit $V_{\mathrm{A}}=$ konstant. . . . . . . . . . 44

3.9 Federkonstantenkennfeld Pneumatik mit $m=$ konstant . . . . . . . . 46

3.10 Federkonstantenkennfeld Hydraulik mit $V_{\mathrm{A}}=$ konstant. . . . . . . . 46

4.1 Prinzip: Hallsensor und magnetoresistiver Sensor. . . . . . . . . . . . 52

4.2 Lineare Bewegung von Hallsensor und Stabmagnet. . . . . . . . . . . 53

4.3 Rotation eines Hallsensors um einen Scheibenmagneten. . . . . . . . 53

4.4 Simulation des Sensorsignals für Ring- und Halbringmagnet. . . . . 55

4.5 Einzelteile des Fingergelenks, Integrationsvorschlag für Winkelsensor. 55

4.6 Sensoranordnungen an der Gelenkaußenseite. . . . . . . . . . . . 56

4.7 Schnittdarstellung der Toleranzen des Fingergelenks. . . . . . . . . . 56

4.8 Achsmittige Sensoranordnungen. . . . . . . . . . . . . . . . . 57

4.9 Varianten liegender Hallsensor an Leiterplatte. . . . . . . . . . . . . . 57

4.10 Entfernungsabhängigkeit der Flussdichte bei Ringmagneten. . . . . . 58

4.11 Entfernungsabhängigkeit der Flussdichte bei Halbringmagneten. . . 59

4.12 Kodierung der Magnetkontur im Genotyp. . . . . . . . . . . . . . 60

4.13 Radienverlauf und Kontur des optimierten Magneten. . . . . . . . . . 62 
4.14 Normale Flussdichte und Positionsfehler nach Optimierung. . . . . . 62

4.15 Prototypen der Varianten A1, B1, C2 und D1. . . . . . . . . . . . . 63

4.16 Aktorteststand zur Vermessung der Sensoren. . . . . . . . . . . . . . 64

4.17 Gemessene Signalverläufe der Prototypen. . . . . . . . . . . . . . . . 65

4.18 Standardabweichung der gemessenen Signalverläufe. . . . . . . . . . 65

4.19 Seiten- und Längsschnitt eines taktilen Drucksensors. . . . . . . . . . 66

4.20 Taktiler Sensor aus leitfähigem Silikon. . . . . . . . . . . . . . . . 67

4.21 Schichtenaufbau von FSR-Sensoren. . . . . . . . . . . . . . . . 67

4.22 Testaufbau für taktile Beschleunigungssensoren. . . . . . . . . . . . 68

4.23 Taktiler Sensor mit Hallsensoren. . . . . . . . . . . . . . . . . . . . . 69

4.24 FPSR- und PVDF-Sensor. . . . . . . . . . . . . . . . . . . . . . . . . . 69

4.25 Fingerspitze mit FPSR-Sensoren. . . . . . . . . . . . . . . . 70

4.26 Touchscreen und resistive Kontaktflächenmessung. . . . . . . . . . . 70

4.27 Array aus druckempfindlichem Gummi mit vernähten Leitern. . . . 72

4.28 Kapazitive Sensorarrays. . . . . . . . . . . . . . . . . . . 72

4.29 Schematische Darstellung optischer Arrays . . . . . . . . . . . . . . . 73

4.30 Betätigung für FSR-Sensoren. . . . . . . . . . . . . . . . . . 74

4.31 Teststand, FSR $F$-R-Kennlinie. $\ldots \ldots \ldots \ldots \ldots \ldots \ldots$

4.32 Quer- und Längsschnitt der FSR-Fingerabdeckung. . . . . . . . . . . . 76

4.33 Sensorabdeckung der Fingerglieder. . . . . . . . . . . . . . . 76

4.34 Sensorabdeckung der Fingerspitze. . . . . . . . . . . . . . . . 76

4.35 FSR $F$-U-Kennlinie, $F$-I-Kennlinie. $\ldots \ldots \ldots \ldots$. . . . . . . . 77

4.36 Doppelt logarithmische FSR $F-R$-Kennlinie und Fehlerkennlinie. . . 78

4.37 Kraftauflösung des FSR-Sensors bei 10 bit Wandlerauflösung. . . . . 79

4.38 Schnittdarstellung der FSR-Anordnung. . . . . . . . . . . . . . . . 79

4.39 Sensor zur Greifkraftmessung mit Klettbandbefestigung. . . . . . . . 80

4.40 Kontaktkraftverteilung FZK-Hand und menschliche Hand. . . . . . . 80

4.41 Kontaktkraftverteilung Otto Bock Prothesen. . . . . . . . . . . . . 81

4.42 Seiten- und Längsschnitt eines taktilen Drucksensors. . . . . . . . . . 82

4.43 Multiaxiale Kraft-Momenten-Sensoren der Karlsruher Hand I und II. 82

4.44 Multiaxialer Kraft-Momenten-Sensor der DLR-Hand II. . . . . . . . . 83

4.45 Zugkraftmessung am Seilzug. . . . . . . . . . . . . . . . . . . 84

4.46 Druckmesszelle für pneumatische Druck- und Positionsregelung. . . 85

4.47 Drucksensor für hydraulischen Betrieb. . . . . . . . . . . . . 86

4.48 Nullmomentenkennlinie und winkelabhängiges Verhalten des Aktors. 87

4.49 Momentenschätzfehler durch Aktormodell. . . . . . . . . . . . . . 88

4.50 Momentenschätzfehler durch Winkel- und Druckmessfehler. . . . . . 88

4.51 Fingervarianten für Verkabelung. . . . . . . . . . . . . . . . . . . . 94

4.52 Leitungssatzvarianten für einen Finger mit drei Gelenken. . . . . . . 98

4.53 Leitungssatzvarianten für den Daumen mit vier Gelenken. . . . . . . 99

5.1 Modell für das Bewegungsverhalten des Gelenks. . . . . . . . . . . . 102

5.2 Viskoelastisches Bewegungsverhalten des Aktors. . . . . . . . . . . . 102

5.3 Pneumatisches System mit kaskadierter Regelung. . . . . . . . . . . . 103

5.4 Schaltbild der pneumatischen Gelenkregelung mit Druckregelventil. 103

5.5 Interner Aufbau des pneumatischen Positionsreglers. . . . . . . . . . 104 
5.6 Eingelenkfinger für den Betrieb des pneumatischen Positionsreglers. 104

5.7 Messung: Positionsregelung eines Gelenks im pneumatischen Betrieb.105

5.8 Pneumatische Gelenkregelung mit diskreter Druckregelung. . . . . . 106

5.9 Streckenmodell für die Druckregelung. . . . . . . . . . . . . . . . 106

5.10 Zeitverhalten eines mit PWM angesteuerten Ventils. . . . . . . . . . . 107

5.11 Kennfelder für $T_{\text {eff }}\left(\Delta p, T_{\text {an }}\right)$ : links Einlassen, rechts Ablassen. . . . . . 108

5.12 Struktur des Druckreglers mit Adaption und FGA. . . . . . . . . . . . 108

5.13 Schematische Darstellung der hydraulischen Gelenkregelung. . . . . 109

5.14 Modell der hydraulischen Strecke. . . . . . . . . . . . . . . . . 110

5.15 Zeitdiskreter Regler für die hydraulische Strecke. . . . . . . . . . . . 110

5.16 Testaufbau für die Momentensteuerung. . . . . . . . . . . . . . . . 111

5.17 Prinzip der Momentenbegrenzung des Positionsreglers. . . . . . . . . 112

5.18 Messung: Positionsregler mit statischer Begrenzung. . . . . . . . . . . 113

5.19 Messung: Positionsregler mit dynamischer Begrenzung. . . . . . . . . 114

5.20 Messung: Positionsregler mit steifigkeitsabhängiger Begrenzung. . . 116

5.21 Hydraulische Regelung mehrerer Aktoren. . . . . . . . . . . . . . 118

5.22 Zustandsautomat für hydraulische Pumpenregelung. . . . . . . . . . 118

5.23 Alternative Multiaktor-Regelung mit reduzierter Ventilanzahl. . . . . 119

5.24 Steuerungsstruktur eines humanoiden Roboters. . . . . . . . . . . . . 120

5.25 Hierarchie der Greifplanung und -koordination. . . . . . . . . . . . 121

5.26 Hierarchie von Handsteuerung und -hardware. . . . . . . . . . . 123

5.27 Hand- und Objektposition während eines Greifvorgangs. . . . . . . . 124

7.1 Gehäusegeometrie des Hallsensors. . . . . . . . . . . . . . . . . 150

7.2 Messverfahren in Drucksensoren. . . . . . . . . . . . . . . . . . . 152

7.3 Leitungssatzvarianten für einen Finger mit zwei Gelenken. . . . . . . 154

7.4 Leitungssatzvarianten für den Daumen mit drei Gelenken. . . . . . . 154 


\section{Tabellenverzeichnis}

4.1 Konfigurationen der Winkelsensorprototypen. . . . . . . . . . . . . 63

4.2 Vergleich von Folienleitertypen. . . . . . . . . . . . . . . . . . . . . 91

4.3 Minimale Biegeradien verschiedener Leitermaterialien. . . . . . . . . 92

4.4 Leitungsverbindung für Verkabelung. . . . . . . . . . . . . . . . . . 94

4.5 Gewichtungstabelle für Vergleichskriterien. . . . . . . . . . . . . . . . 98

4.6 Bewertung der Leitungssatzvarianten für Finger mit drei Gelenken. . 99

4.7 Bewertung der Leitungssatzvarianten für Daumen mit 4 Gelenken. . 100

5.1 Schätzfehler der statischen Momentenbegrenzung . . . . . . . . . . 113

5.2 Schätzfehler der dynamischen Momentenbegrenzung . . . . . . . . . 115

5.3 Schätzfehler der steifigkeitsgesteuerten Momentenbegrenzung . . . . 117

5.4 Arm- und Handaktivitäten eines beispielhaften Greifablaufs. . . . . . 124

7.1 Bewertung der Leitungssatzvarianten für Finger mit zwei Gelenken. 153

7.2 Bewertung der Leitungssatzvarianten für Daumen mit drei Gelenken.155 


\section{Anhang}

\subsection{Technische Daten der Sensoren}

\subsubsection{Hallsensoren}

Technische Daten nach [7]:

Hersteller

Typenbezeichnung

Gehäusetyp

Gehäusemaße

Versorgungsspannung

Max. normale Flussdichte

Allegro Microsystems

Ausgangssignal

Temperaturbereich

Gewicht

Einzelpreis für Muster

A3516EUA

3-Pin Ultra-Mini SIP 'UA'

$4,2 \times 3,1 \times 1,6 \mathrm{~mm}^{3}$

$U_{\mathrm{V}}=5 \mathrm{~V} \pm 0,5 \mathrm{~V}$

$B_{n, \max }= \pm 0.1 \mathrm{~T}$

$U_{\mathrm{A}}=\frac{U_{\mathrm{V}}}{2}\left(1+\frac{B_{\mathrm{n}}}{0.2 T}\right)$

$-40^{\circ} \mathrm{C}$ bis $+85^{\circ} \mathrm{C}$

$0,1 \mathrm{~g}$

EUR 3,10 bei Abnahme von $25 \mathrm{St}$.

Alternativtyp: A3515EUA mit der doppelten Empfindlichkeit:

Max. normale Flussdichte

Ausgangssignal

$B_{n, \max }= \pm 0.05 T$

$U_{\mathrm{A}}=\frac{U_{\mathrm{V}}}{2}\left(1+\frac{B_{\mathrm{n}}}{0.1 T}\right)$
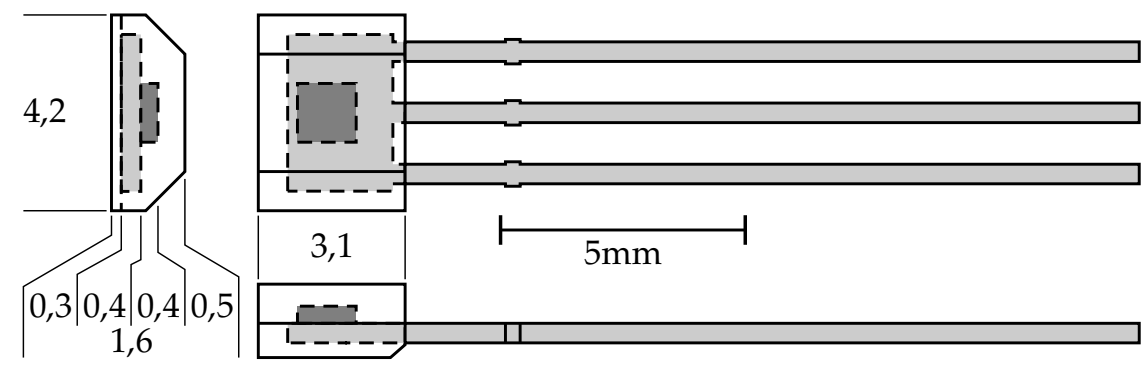

Abbildung 7.1: Gehäusegeometrie des Hallsensors, die Kontakte sind hellgrau, das Sensorelement ist dunkelgrau angedeutet.

Die Vorderseite des Sensors ist beschriftet und an zwei seitlichen Fasen erkennbar. Eine weitere Fase befindet sich an der Anschlussseite des Rückens. 


\subsubsection{Magnete}

Der ringförmige Seltenerdmagnet ist eine Sonderanfertigung mit den folgenden Parametern:

Lieferant

Materialbezeichnung

Material

Magnetisierung

Oberflächenbeschichtung

Geometrie

Energieprodukt

Remanenz

Koerzitivfeldstärke

relative permanente Permeabilität

Dichte

max. Einsatztemperatur

Gewicht

Einzelpreis für Muster
M\&E Magnettechnik Elektromechanik, Remscheid, DE

ME30Zn

NdFeB (Neodym-Eisen-Bor)

diametral

Zink

Außendurchmesser $d_{a}=5 \mathrm{~mm}$

Innendurchmesser $d_{i}=2,5 \mathrm{~mm}$

Dicke $t=1 \mathrm{~mm}$

$(B \cdot H)_{\max }=223 \frac{\mathrm{kJ}}{\mathrm{m}^{3}}$

$B_{r} \geq 1080 m T$

${ }_{B} H_{c} \geq 780 \frac{\mathrm{kA}}{\mathrm{m}}$

${ }_{J} H_{c} \geq 955 \frac{\mathrm{kA}}{\mathrm{m}}$

$\mu_{p}=1,05$

$\varrho_{M E 30}=7,5 \frac{g}{c m^{3}}$

$80^{\circ} \mathrm{C}$

$0,1 \mathrm{~g}$

EUR 1,55 bei Abnahme von 50 St.

\subsubsection{IEE FSR-Sensoren}

Allgemeine technische Daten für FSR-Sensoren nach [78]:

Hersteller

Substrat

Betriebstemperatur

Temperaturkoeffizient

Betriebsspannung

Maximale Stromdichte

Maximale Verlustleistung

Technische Daten für FSR-149:

Außendurchmesser

Länge ohne Kontakte

Breite der Kontaktierung

Dicke

Durchmesser der aktiven Fläche

Aktive Fläche

Maximalstrom

Gewicht

Einzelpreis für Muster
IEE International Electronics \& Engineering, Luxemburg Polyätherimid, Polyäthersulfon, Polyester

$-40^{\circ} \mathrm{C} \ldots+85^{\circ} \mathrm{C}$

$-0,8 \frac{\%}{K}$

$1 \ldots 5 \mathrm{~V}$

$1 \frac{m A}{c m^{2}}$

$1 \mathrm{~mW}$

$7,62 \mathrm{~mm}$

$38,1 \mathrm{~mm}$

$6,35 \mathrm{~mm}$

$0,34 \mathrm{~mm}$

$4,03 \mathrm{~mm}$

$12,7 \mathrm{~mm}^{2}$

$0,13 m A$

$0,1 \mathrm{~g}$

EUR 7,49 bei Abnahme von 50 St. 


\subsubsection{Drucksensoren}

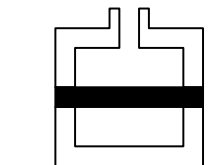

Absolutdruck Messung gegenüber Referenz (Vakuum) (absolute)

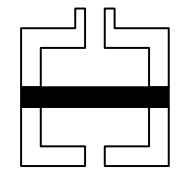

Relativdruck Messung gegenüber Umgebungsdruck (gauge)

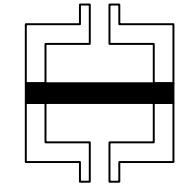

Differenzdruck Messung gegenüber zweitem Anschluss (differential)

Abbildung 7.2: Messverfahren in Drucksensoren.

\section{Keller Serie 1 TAB}

Kenndaten der Keller-Drucksensoren nach [97].

Hersteller

Typenbezeichnung

Trägermaterial

Gewicht

Messverfahren

Konfiguration

Brückenwiderstand

Nennstrom

Messbereich

Empfindlichkeit

Linearitätsfehler

Betriebstemperatur

Temperaturverhalten

Einzelpreis für Muster
Keller AG, Winterthur, Schweiz

Serie $1 \mathrm{TAB}$

Polyimid

$2 g$

Absolut

Wheatstone-Brücke

$3,5 \pm 0,7 k \Omega$

$4 \mathrm{~mA}$

$0 \ldots 10 \cdot 10^{5} \mathrm{~Pa}$

typ. $90 \frac{m V}{10^{5} P a}$ bei Nennstrom

$\max .1 \%$

$-20^{\circ} \mathrm{C} \ldots+100^{\circ} \mathrm{C}$

Nullpunktverschiebung max. $0,1 \frac{\mathrm{mV}}{\mathrm{K}}$

Empfindlichkeitsänderung max. $0,025 \frac{\%}{K}$ 15 EUR 


\section{Honeywell 24PC}

Kenndaten der Honeywell-Drucksensoren nach [74].

Hersteller

Serien-/ Typenbezeichnung

Dichtungsmaterialien

Gewicht

Messverfahren

Konfiguration

Brückenwiderstand

Nennspannung

Messbereich

Maximaldruck

Empfindlichkeit

Linearitätsfehler

Betriebstemperatur

Einschwingdauer

Gewicht

Einzelpreis für Muster
Honeywell Inc., Freewell, Illinois, USA

24PC / 24PCGFM6G

EPDM, Fluorosilikon, Neopren, Silikon

$2 g$

Relativ

Wheatstone-Brücke

$5 k \Omega$

typ. $10 \mathrm{~V}$

$\pm 17,5 \cdot 10^{5} \mathrm{~Pa}$

$\pm 35 \cdot 10^{5} \mathrm{~Pa}$

typ. $12 \frac{m V}{10^{5} P a}$ bei Nennspannung

max. $1 \%$

$-40^{\circ} \mathrm{C} \ldots+85^{\circ} \mathrm{C}$

$1 \mathrm{~ms}$

$2 g$

30 EUR bei Abnahme von 25 St.

\subsection{Verkabelung}

\subsubsection{Finger mit zwei Freiheitsgraden}

Abbildung 7.3 zeigt die Leitungssatzvarianten für den Finger mit zwei Gelenken. Ihre Bewertung erfolgt in Tabelle 7.1. Für den Vergleich mit der Bewertung des Fingers mit drei Gelenken wird auf Abschnitt 4.4.5 verwiesen.

\begin{tabular}{l|cc|cc|rc|rc|rc|rc|} 
Variante & $\mathrm{a}$ & $\mathrm{b}$ & $\mathrm{c}$ & $\mathrm{d}$ & $\mathrm{e}$ & $\mathrm{f}$ & \\
\hline$n_{\mathrm{K}}$ & 28 & - & 20 & ++ & 20 & ++ & 22 & + & 22 & + & 30 & -- \\
$v_{\mathrm{K}}$ & 75 & - & 55 & ++ & 55 & ++ & 63 & 0 & 59 & + & 81 & -- \\
$s_{\mathrm{K}}$ & 16 & 0 & 32 & -- & 8 & ++ & 8 & ++ & 8 & ++ & 22 & - \\
$s_{\mathrm{L}}$ & 10 & - & 10 & - & 2 & ++ & 2 & ++ & 2 & ++ & 2 & ++ \\
$l_{\mathrm{F}}$ & 14 & - & 13 & 0 & 9 & ++ & 10 & + & 10 & + & 17 & -- \\
\hline$b$ & 0,72 & 0,50 & 0,00 & 0,12 & 0,11 & \multicolumn{2}{|c|}{0,75} \\
\hline Rang & 5 & \multicolumn{2}{|c|}{4} & \multicolumn{2}{|c|}{1} & \multicolumn{2}{|c|}{3} & \multicolumn{2}{|c|}{2} & \multicolumn{2}{|c|}{6} \\
\hline
\end{tabular}

Tabelle 7.1: Bewertung der Leitungssatzvarianten für Finger mit zwei Gelenken. 
a)

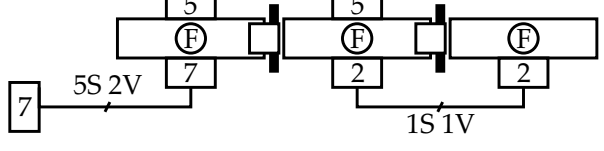

c)
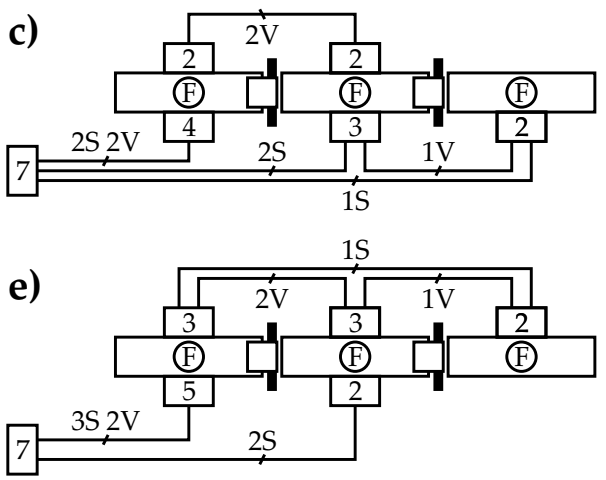

b)

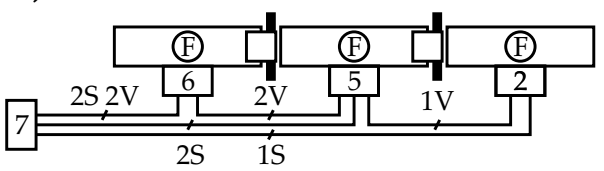

d)

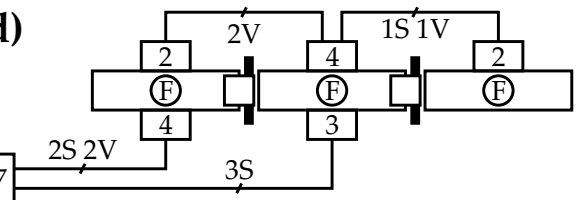

f)

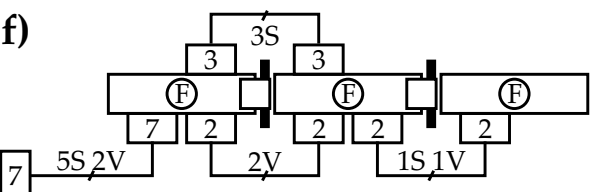

Abbildung 7.3: Leitungssatzvarianten für einen Finger mit zwei Gelenken.

\subsubsection{Daumen mit drei Freiheitsgraden}

Die Leitungssatzvarianten für den Daumen mit drei Gelenken sind in Abbildung 7.4 dargestellt. Die Bewertung ist in Tabelle 7.2 zusammengefasst. Verglichen werden die Ergebnisse mit denen des Daumens mit vier Freiheitsgraden bereits im Abschnitt 4.4.6.

a)

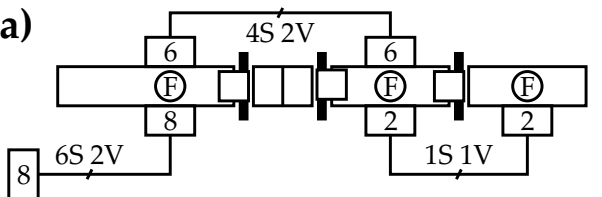

c)

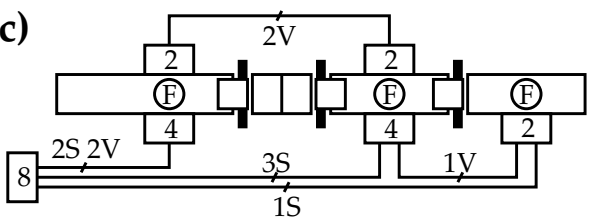

e)

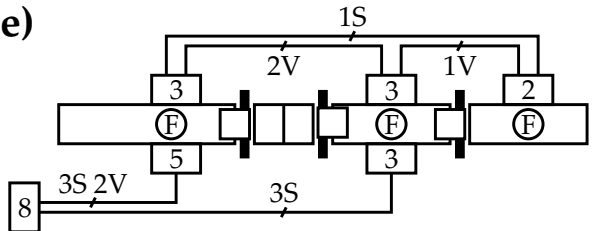

b)

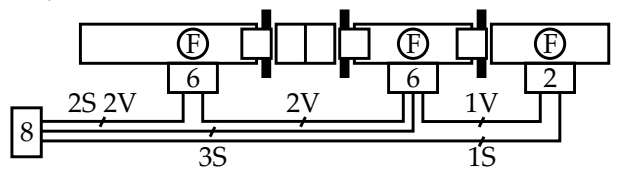

d)
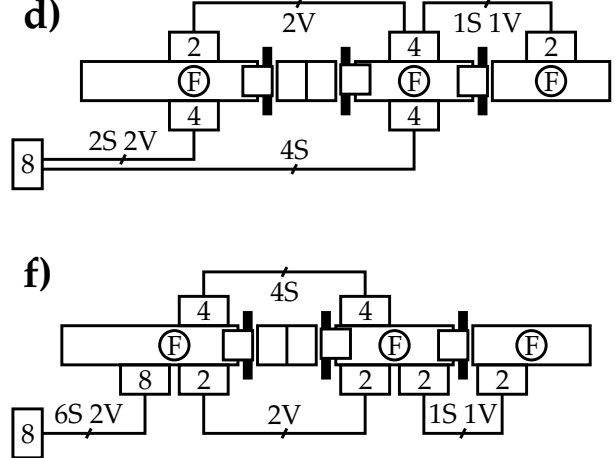

Abbildung 7.4: Leitungssatzvarianten für den Daumen mit drei Gelenken. 


\begin{tabular}{l|rl|rl|rc|rc|rc|rc|} 
Variante & $\mathrm{a}$ & $\mathrm{b}$ & $\mathrm{c}$ & $\mathrm{d}$ & $\mathrm{e}$ & $\mathrm{f}$ & \\
\hline$n_{\mathrm{K}}$ & 32 & - & 22 & ++ & 22 & ++ & 28 & 0 & 24 & + & 34 & -- \\
$v_{\mathrm{K}}$ & 132 & 0 & 100 & ++ & 100 & ++ & 136 & - & 104 & + & 142 & - \\
$s_{\mathrm{K}}$ & 36 & - & 60 & - & 20 & + & 24 & 0 & 4 & ++ & 22 & + \\
$s_{\mathrm{L}}$ & 36 & - & 36 & - & 12 & 0 & 12 & 0 & 0 & ++ & 12 & 0 \\
$l_{\mathrm{F}}$ & 16 & - & 14 & 0 & 10 & ++ & 14 & 0 & 10 & ++ & 18 & -- \\
\hline$b$ & 0,80 & 0,50 & 0,10 & 0,48 & 0,05 & 0,78 \\
\hline Rang & 6 & 4 & 2 & 3 & 1 & 5 \\
\hline
\end{tabular}

Tabelle 7.2: Bewertung der Leitungssatzvarianten für Daumen mit drei Gelenken.

\subsection{Genetischer Algorithmus}

\section{Hauptprogramm}

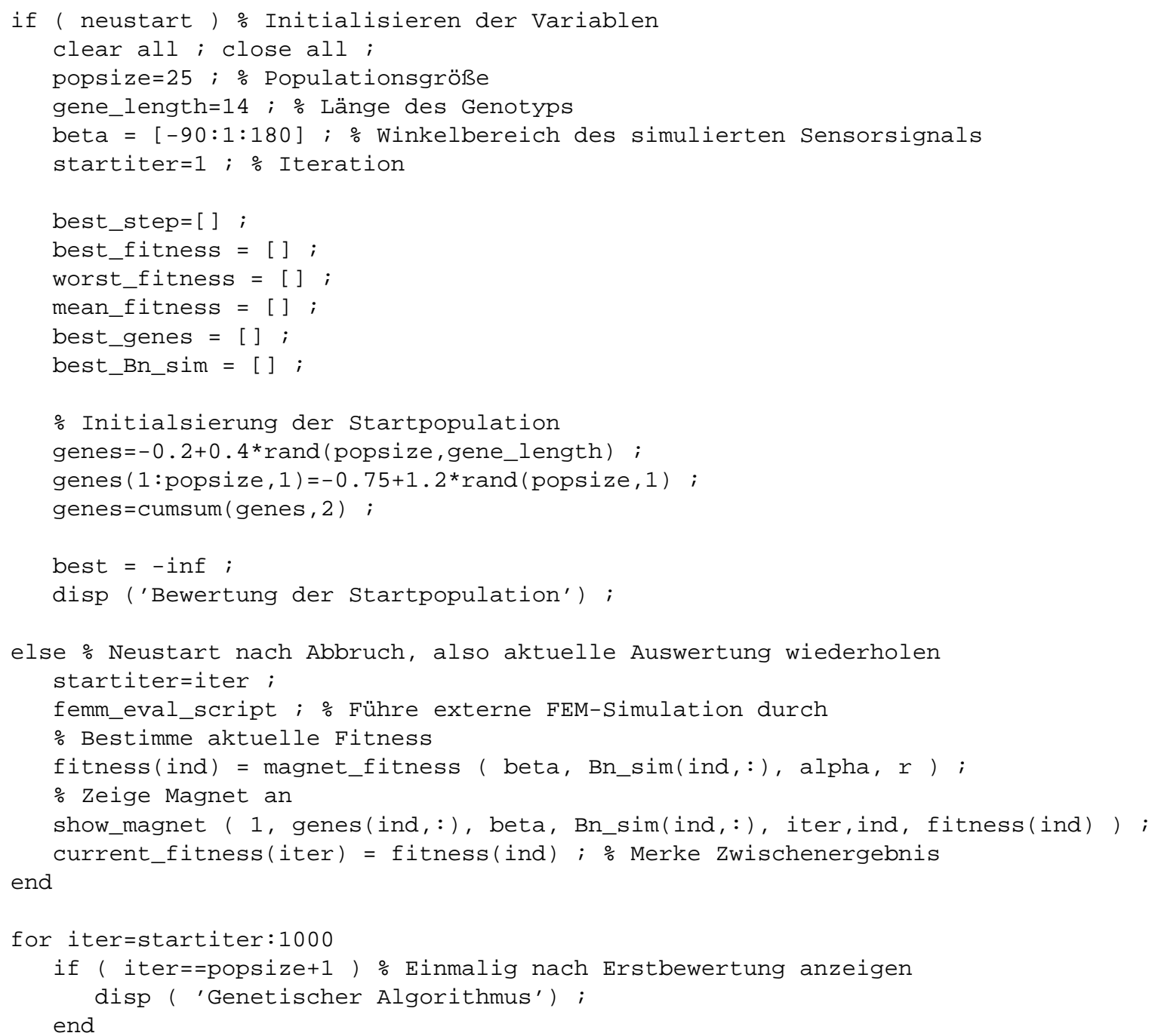




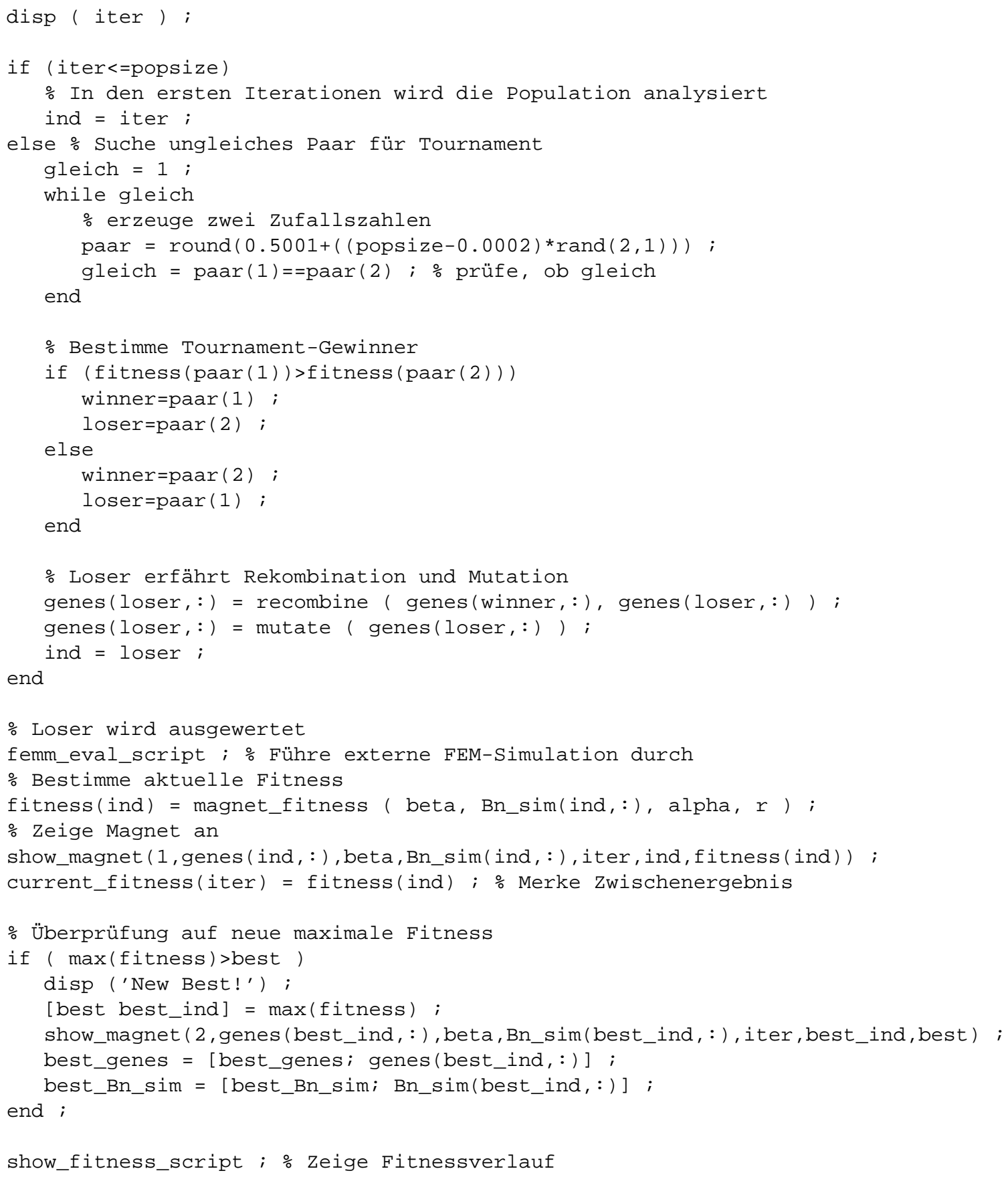




\section{Rekombination}

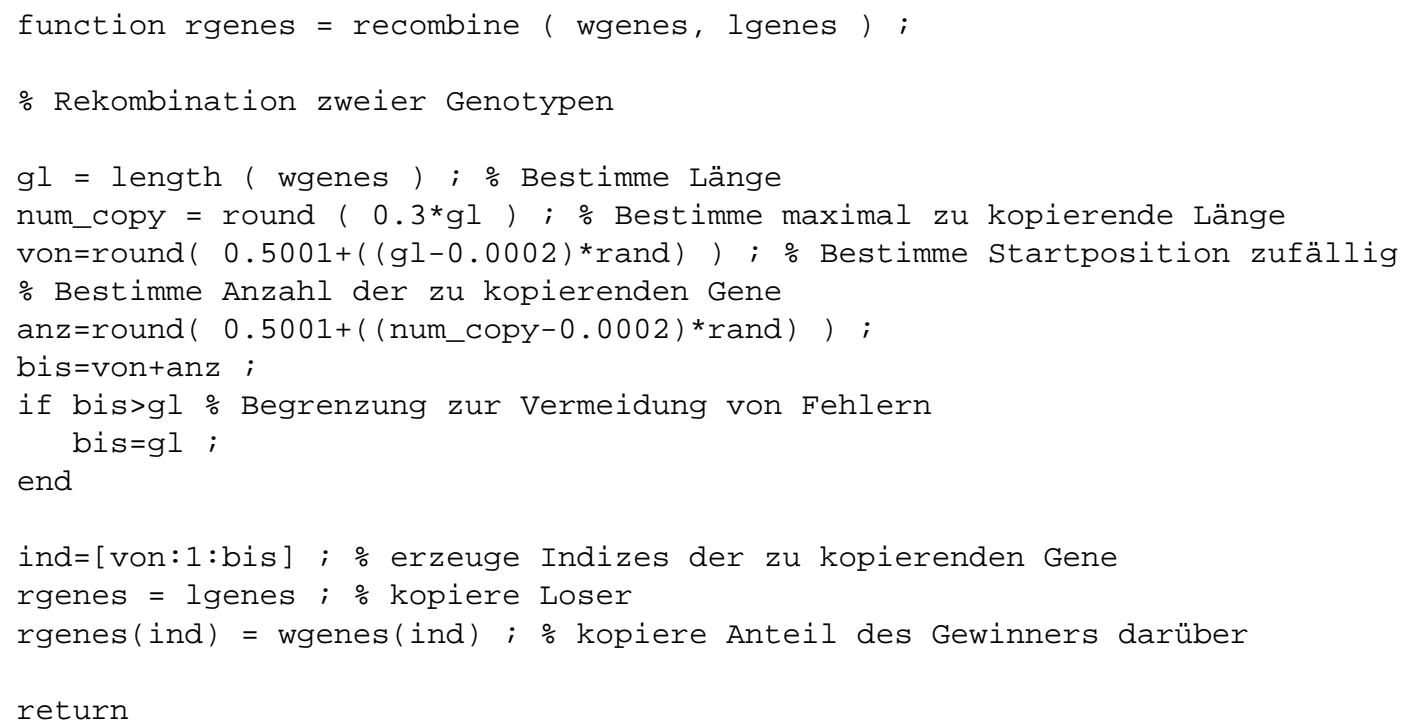

\section{Mutation}

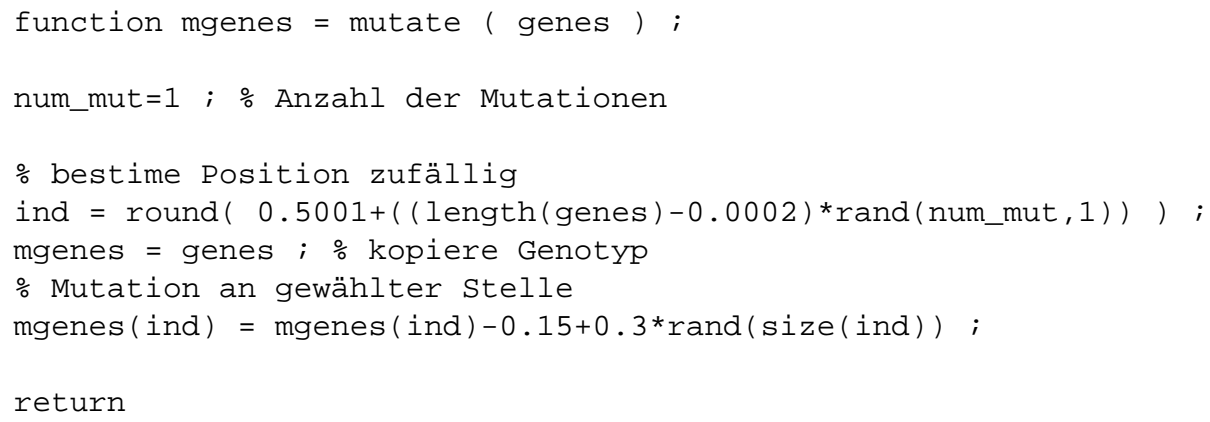

